\title{
Advanced methods for quality assurance and dose distribution improvement for high dose rate brachytherapy
}

Citation for published version (APA):

Bellezzo, M. (2020). Advanced methods for quality assurance and dose distribution improvement for high dose rate brachytherapy. [Doctoral Thesis, Maastricht University]. Gildeprint en Universitaire Pers Maastricht. https://doi.org/10.26481/dis.20200611mb

Document status and date:

Published: 01/01/2020

DOI:

10.26481/dis.20200611mb

Document Version:

Publisher's PDF, also known as Version of record

Please check the document version of this publication:

- A submitted manuscript is the version of the article upon submission and before peer-review. There can be important differences between the submitted version and the official published version of record.

People interested in the research are advised to contact the author for the final version of the publication, or visit the DOI to the publisher's website.

- The final author version and the galley proof are versions of the publication after peer review.

- The final published version features the final layout of the paper including the volume, issue and page numbers.

Link to publication

\footnotetext{
General rights rights.

- You may freely distribute the URL identifying the publication in the public portal. please follow below link for the End User Agreement:

www.umlib.nl/taverne-license

Take down policy

If you believe that this document breaches copyright please contact us at:

repository@maastrichtuniversity.nl

providing details and we will investigate your claim.
}

Copyright and moral rights for the publications made accessible in the public portal are retained by the authors and/or other copyright owners and it is a condition of accessing publications that users recognise and abide by the legal requirements associated with these

- Users may download and print one copy of any publication from the public portal for the purpose of private study or research.

- You may not further distribute the material or use it for any profit-making activity or commercial gain

If the publication is distributed under the terms of Article $25 \mathrm{fa}$ of the Dutch Copyright Act, indicated by the "Taverne" license above, 
Advanced methods for quality assurance and dose distribution improvement for high dose rate brachytherapy

Murillo Bellezzo 


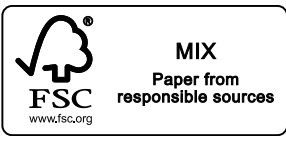

Printed by: $\quad$ Gildeprint - Enschede

ISBN: $\quad$ 978-94-6402-308-4

(C) copyright Murillo Bellezzo, Maastricht 2020

All rights reserved. No part of the material protected by this copyright notice may be reproduced or utilised in any form or by any means, electronic or mechanical, including photocopying, recording or by any information storage and retrieval system, without written permission from the author and the publisher(s) holding the copyrights of the reprinted article(s). 


\section{Advanced methods for quality assurance and dose distribution improvement for high dose rate brachytherapy}

Thesis

To obtain the degree of Doctor at Maastricht University, on the authority of the Rector Magnificus, Prof.dr. Rianne M. Letschert, in accordance with the decision of the Board of Deans, and the degree of Doctor at the University of São Paulo, on the authority of the Rector Magnificus, Prof.dr. Vahan Agopyan, to be defended in public

on Thursday 11th of June 2020 at 10:00 hours, in Maastricht and on Monday 22nd of June 2020 at 13:30 hours, in São Paulo

by

Murillo Bellezzo 


\section{Supervisors}

Prof. Dr. Frank Verhaegen

Prof. Dr. Hélio Yoriyaz, University of São Paulo

\section{Co-supervisor}

Prof. Dr. Brigitte Reniers, Associate professor, Universiteit Hasselt

Prof. Dr. Gabriel P. Fonseca, Assistant professor, Maastricht University

\section{Assessment Committee - the Netherlands}

Prof. Dr. Dirk De Ruysscher (chairman), Maastricht University

Prof. Dr. em. Erik van Limbergen, Dept Radiotherapie-oncologie, UZ Leuven

Prof. Dr. Kari Tanderup, Associate professor, Aarhus University Hospital, Denmark

Dr. Mirko Unipan, Maastro

\section{Assessment Committee - Brazil}

Prof. Dr. Hélio Yoriyaz (chairman),University of São Paulo

Prof. Dr. Gabriel P. Fonseca, Assistant professor, Maastricht University

Prof. Dr. Marco A. R. Fernandes, Universidade Estadual Paulista

Prof. Dr. Paulo T. D. Siqueira, Instituto de Pesquisas Energéticas e Nucleares

Dr. Paula C. G. Antunes, Universidade São Judas Tadeu 
In memory of my beloved mother 



\section{Acknowledgements}

I would like to acknowledge my supervisors, Hélio, Frank, Gabriel and Brigitte. Thank you for the guidance, dedication, for patience, for the patience again, for reminding me of the deadlines, for lying about the deadlines so I would not miss the real deadline. Have I thanked you for the patience? There is no doubt in my mind that without you I wouldn't finish my PhD. Also, thank you for the gift that was the Flower project, something that fell in my lap and became the focus of my $\mathrm{PhD}$.

Talking about the Flower, Maaike and Evert, thank you for letting me be part of this project and Maaike, I am still upset because I couldn't get your jump scare on camera when the pig moved. Evert, I've never seen someone with such positive attitude as you, always caring a smile on your face.

Celine, Erik and Robert, thank you for all the help, and Robert, thank you for showing me that I suck at cycling.

Gabriel, you made it possible for me to come to Netherlands, you literally opened the doors of your house for me, you were there for almost all the experiments that I've done, you told me when my work was bad, and you also introduced me to some of my most expensive hobbies (I am not sure if I should thank you for that last one). Lou, thank you for having me over so many times, I am really happy to have you around.

Matthijs, small gestures may change someone's day, thank you for the Coca-Cola, and for being such a nice guy.

Mark and Shane, you guys are the reason why I believe that Irish people are the best in the world (after brazilians, of course). Isabel, you are the reason why I think portuguese people are the worst! No, you know that's not true, you are one of the best people I have ever had the pleasure of meeting and I will be always cheering for you.

Brent, I am really relieved that my defense will be before yours, otherwise you would have set the bar too high. I still don't understand why you find me funny, but I will take it as a compliment. You were also responsible to making me want a Mac, and I blame you for that.

Cecile, you are a sweet person who was always there when I had a question, no wonder why you are so good at quizzes!

Ana, I am afraid of the things that you remember. It's impressive how you genuinely want to know how people are doing and you (almost) always make time to talk. Also, you are 
always checking on me to see if I am still alive and my house is basically furnished with your stuff. So, I just want to thank you for everything.

Those who know me also know that I have a memory of a goldfish and I live in my own bubble with my noise cancelling headphones. I am sure that I will forget someone if I start mentioning people individually. Therefore, I would like to thank everyone who was part of my $\mathrm{PhD}$ journey and everyone who was probably ignored while inviting me for a coffee-break or for lunch (remember of the bubble thing).

I also must thank my friends who, even living in other countries, were always there to talk with me when I needed, Na, Bárbara and Raíza. You girls are among the most precious things in my life.

Lastly, I would like to thank my family for all the support, and my mother, who always believed in me, even when I didn't. 


\section{Summary}

Brachytherapy is a method of radiotherapy that consists on placing a radioactive source in close contact or inside the tumor, resulting in a steep gradient on the dose distribution, which helps protecting organs at risk but also makes the technique prone to errors in case of wrong positioning of the radioactive source. There are several stages involved in a treatment workflow, from afterloader commissioning to treatment delivery and verification. In this thesis, several stages of a brachytherapy treatment were evaluated as part of an effort to improve accuracy and precision of dose delivery.

The viability to 3D tracking a brachytherapy source using an imaging panel was shown possible by applying a $2 \mathrm{D}$ gaussian fit to the panel response to locate the source and the distance between the source and the panel could be determined considering the gaussian shape. The same principle was adopted in a quality assurance system for brachytherapy applicators that uses an ${ }^{192}$ Ir source to acquire a projection of the applicator into the imaging panel and then sends the source inside the applicator to track its movement with submillimeter precision.

The main focus of this thesis, however, was to develop an applicator for rectal cancer capable of generating a dose distribution similar to those generated by $50 \mathrm{kVp}$ x-ray devices, but using HDR ${ }^{192}$ Ir sources. The applicator was successfully modeled by using the inverse square law to achieve a steep dose falloff by bringing the ${ }^{192}$ Ir source at several points in close contact to the treatment surface.

Finally, a new afterloading system was thoroughly evaluated using multiple quality assurance devices, high speed camera and imaging panel to verify current limitations in brachytherapy dose delivery due to precision and accuracy of dwell times, dwell positions and transit dose effects.

Keywords: brachytherapy, Monte Carlo, dosimetry, commissioning 



\section{Resumo}

Braquiterapia é um método de radioterapia que consiste em posicionar uma fonte radioativa próxima ou dentro do tumor, resultando em uma distribuição de dose com gradiente acentuado, o que ajuda a proteger os órgãos de risco, mas também torna a técnica propensa a erros caso a fonte seja posicionada incorretamente. Existem várias etapas envolvidas no processo da braquiterapia, desde o comissionamento do afterloader até a realização e verificação do tratamento.

Nesta tese, várias etapas da braquiterapia foram avaliadas como parte de um esforço para melhorar a exatidão e precisão da administração da dose. A viabilidade do rastreamento 3D de uma fonte de braquiterapia usando um painel de imagem foi demonstrada possível pela aplicação de um ajuste gaussiano $2 \mathrm{D}$ à resposta do painel para localizar a fonte, $\mathrm{e}$ a distância entre a fonte e o painel pode ser determinada considerando o formato da gaussiana. O mesmo princípio foi adotado em um sistema de controle de qualidade para aplicadores de braquiterapia que usa uma fonte ${ }^{192}$ Ir para obter a projeção do aplicador no painel de imagem e em seguida envia a fonte dentro do aplicador para rastrear seu movimento com precisão submilimétrica.

O foco principal desta tese, no entanto, foi o desenvolvimento de um aplicador de câncer retal que usa fontes HDR ${ }^{192}$ Ir para gerar uma distribuição de doses semelhante à gerada por dispositivos de raios-x de $50 \mathrm{kVp}$. O aplicador foi modelado com sucesso usando a lei do inverso do quadrado da distância para obter uma elevada atenuação da taxa de dose ao posicionar a fonte ${ }^{192} \mathrm{Ir}$ em vários pontos próximos à superfície de tratamento.

Finalmente, um novo afterloader foi avaliado usando vários dispositivos de controle de qualidade, câmera de alta velocidade e painel de imagem para verificar as limitações atuais na administração de doses em braquiterapia devido à precisão e exatidão dos tempos de parada, posições de parada e efeitos da dose de trânsito.

Palavras chave: braquiterapia, Monte Carlo, dosimetria, commissionamento 



\section{Contents}

Acknowledgements $\quad$ i

Summary

Resumo $\quad$ v

$\begin{array}{ll}\text { List of Figures } & \text { ix }\end{array}$

List of Tables $\quad$ xvii

List of abbreviations $\quad$ xix

Chapter 1 Introduction 1

$\begin{array}{lll}\text { Chapter } 2 & \text { Objectives } & \mathbf{7}\end{array}$

2.1 Pretreatment Verification System . . . . . . . . . . . . . . . . . . . . . . . . . .

2.2 Iridium Imaging System (IRIS) $\ldots \ldots \ldots \ldots \ldots \ldots$

2.3 Designing A Rectal Applicator For Contact Brachytherapy, A Proof Of Concept 11

2.4 Advanced Design Of A Rectal Applicator For Contact Brachytherapy . . . . . 12

2.5 Mechanical Evaluation Of The Bravos Brachytherapy System . . . . . . . . . 12

2.6 Transit Time . . . . . . . . . . . . . . . . . . . . . 13

$\begin{array}{lll}\text { Chapter } 3 & \text { Literature Review } & 15\end{array}$

Chapter 4 Materials and Methods 21

4.1 Pretreatment Verification System ～. . . . . . . . . . . . . . . . . . . . 23

4.2 Iridium Imaging System (IrIS) $\ldots \ldots \ldots$. . . . . . . . . . . . . 30

4.3 Designing a rectal applicator for contact brachytherapy, a proof of concept . 35

4.4 Advanced Design Of A Rectal Applicator For Contact Brachytherapy . . . . 39

4.5 Mechanical Evaluation Of The Bravos Brachytherapy System ～. . . . . . . . 47

4.6 Transit Time . . . . . . . . . . . . . . . . . . . . . . . . . 54

Chapter 5 Results and Discussion $\quad \mathbf{5 9}$

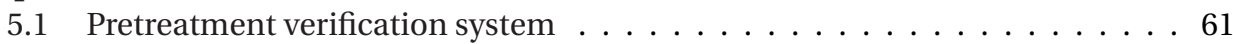

5.2 Iridium Imaging system (IrIS) $\ldots \ldots \ldots \ldots$. . . . . . . . . . . 72 
5.3 Designing a rectal applicator for contact brachytherapy, a proof of concept . 74

5.4 Advanced design of a rectal applicator for contact brachytherapy . . . . . . . 83

5.5 Mechanical evaluation of the Bravos brachytherapy system . . . . . . . . . 89

5.6 Transit time . . . . . . . . . . . . . . . . . . . 97

$\begin{array}{lll}\text { Chapter } 6 & \text { Conclusions } & 107\end{array}$

$\begin{array}{lll}\text { Chapter } 7 & \text { Future Perspectives } & 115\end{array}$

$\begin{array}{lll}\text { Appendix A Nonlinearity Correction } & 121\end{array}$

Appendix B Dwell Position Verification $\quad 125$

Appendix C Monte Carlo Simulations Parameters And Uncertainty Analysis $\quad 129$

$\begin{array}{ll}\text { References } & 135\end{array}$

$\begin{array}{ll}\text { Valorization } & 151\end{array}$

$\begin{array}{ll}\text { List of Publications } & 157\end{array}$

$\begin{array}{ll}\text { Curriculum vitae } & 163\end{array}$ 


\section{List of Figures}

1.1 Overview of standard practices for BT treatments workflow and most relevant advanced

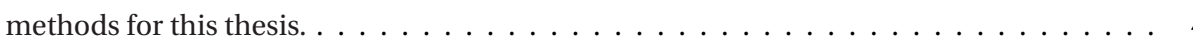

4.1 a) experimental setup using a robotic arm, a GammaMedplus iX afterloader $\left({ }^{192} \mathrm{Ir}\right.$ source), an IP, ultrasonic sensors and the water phantom employed for most of the experiments; b) detail of the three ultrasonic sensors (arrows) used in the experimental setup. Coordinate system: xy measurement plane; $\mathrm{z}$ distance between the source and the panel. . . . . . . . . . . . . . . . . . . . . . . . . . . . . . 24

4.2 Experimental setup used to evaluate clinical scenarios and simulated errors. a) gynecological applicator (red arrow) with 5 needles. b) experimental setup showing the IP, water phantom, gynecological applicator and the robotic arm. . . . . . . . . . . . . 29

4.3 Picture of the IrIS system. A 3D printed baseplate with several radiopaque markers used to scale and register images acquired by the IP. Images are acquired using the photons emitted by the HDR ${ }^{192}$ Ir source inside the imaging channel and each applicator has its own 3D printed holder. . . . . . . . . . . . . . . . . . . . . . . . . . . . . 32

4.4 Scaled BG image showing measured distances between reference points [measured (expected)], and the projection casted by the vertical markers coincident with the measurement lines (meaning that the BT source is in the correct position inside the

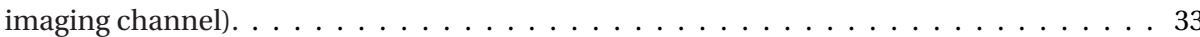

4.5 Image generated by the IrIS system showing the reference points used for registration, planned source path and planned dwell positions (imported from the TPS). . . . . . . . . 34

4.6 Image of the applicator after BG subtraction showing a) Source path and planned dwell positions; b) Measured dwell positions on top of planned dwell positions. . . . . . . . . . . 34

4.7 Model for the multi-channel HDR contact applicator: A central core holding a number of straight channels positioned in cylindrical patterns surrounded by lateral shielding with a plastic cap on top to stop secondary electrons. The tip of the source $(2.5 \mathrm{~mm}$ from the

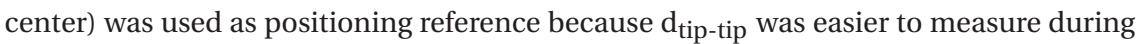

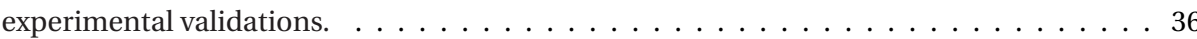


4.8 a) Example of a film measurement with an asymmetrical dose distribution, irradiated at $10 \mathrm{~mm}$ depth. A dose profile was measured along the dashed line intercepting the lower dose region with an angle $\phi$; b) The resulting dose profile has 2 peaks with a difference between the maxima of $\Delta \mathrm{D}_{(\%)}$. The angle $\phi_{\max }$ is where $\Delta \mathrm{D}_{(\%)}$ is highest ( $\Delta \mathrm{D}_{\% \max }$ ); $\mathrm{c}$ ) With $\phi_{\max }$ and $\Delta \mathrm{D}_{\% \max }$ it is possible to derive the orientation $\theta_{\mathrm{s}}$ and $\phi$ of the source by comparison with MC simulations. . . . . . . . . . . . . . . .

4.9 Model of the MAASTRO applicator prototype built for experimental validation. a) $3 \mathrm{D}$ view of the applicator with the contact surface highlighted. The contact surface geometric center $(\mathrm{Gc})$ is represented by the intersection between lines L1 and L2. However, since the most distal dwell positions of each channel do not reach the edge of the applicator, a shift is anticipated between Gc and the center of the dose distribution (Dc), represented by the intersection between lines L2 and L3; b) Top view of the applicator, showing that there is a minimum distance (d) of approximately $0.5 \mathrm{~mm}$ between the catheter and the lateral wall of the applicator because the channel (not shown) to guide the catheter is slightly thicker than the catheter and there is also a minimum wall thickness that can be 3D printed, resulting in an applicator with $20.8 \mathrm{~mm}$ diameter core with a maximum distance of $18 \mathrm{~mm}$ between the side catheters along the smaller diameter of the contact surface and ; c) Cross section along dashed line on (b) of the applicator inserted into the proctoscope, with a inset showing a perpendicular view of the contact surface, highlighting its high dose region. The proctoscope has a 1.5 mm thick tungsten alloy (Inermet IT180, $=18.0 \mathrm{~g} / \mathrm{cm}^{3}$ ) shielding encapsulated by $0.5 \mathrm{~mm}$ thick plastic caps. This is done to avoid corrosion of the shielding and stop secondary

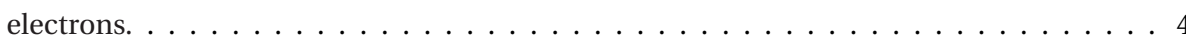

4.10 a) Experimental setup for dose measurement at different depths in solid water. The applicator was held in position by a 3D printed holder with several deg of freedom to ensure that the contact surface of the applicator was in contact with the solid water surface. Films were placed flat between $25 \mathrm{~cm}$ of solid water and a top solid water slab to define scoring depth. The shielding (inset) was purposely not fixed to the applicator, so it would be possible to shift It along the applicator to verify if the contact surface was flush with the solid water before putting the shielding back in position for irradiation. b) Experiments for dose leakage measurements around the shielding were performed with the applicator axis slightly inclined from the vertical axis $(\sim 20 \mathrm{deg})$ to reduce transfer guide tubes curvature. The shielding was fixed around the applicator and the film was held between 2 concentric 3D printed cylinders (inset) made of PLA (Polylactic acid, $\left.=1.2 \mathrm{~g} / \mathrm{cm}^{3}-\left(\mathrm{C}_{3} \mathrm{H}_{4} \mathrm{O}_{2}\right)_{n}\right)$, cylinder 1 would snugly fit around the shielding, defining the scoring distance, while the second cylinder would keep the film in place during irradiation. 46 
4.11 a) The ideal distance between the CamScale and the afterloader is $50 \pm 1.5 \mathrm{~cm}$ (measured horizontally at the laser origin height), achieved by laser alignment. The inset shows the laser projected onto the afterloader used for alignment. For a proper alignment the laser projection should be inside the region defined by two markers (visible in the insert figure). b) Schematic drawing showing the position of the cameras and ruler used for position verification in the CamScale. . . . . . . . . . . . . . . . 50

4.12 Schematic drawing of the mechanism that directs the indexer track tube towards the channel used for irradiation. The indexer track tube is directed to channel 1 by default. . 51

4.13 Differences in dwell time calculations between a) GammaMedplus iX and b) Bravos

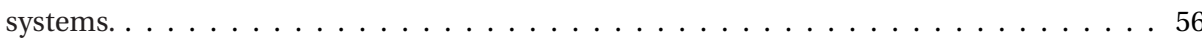

4.14 Example of source movement prediction with and without transit time correction. The vertical dashed line represents the separation between the model when the source is moving from the afterloader to the most distal dwell position and when the source is moving between dwell positions. The source movement is initially predicted without transit time correction, then the transit time for each dwell position is calculated and removed from the dwell time. . . . . . . . . . . . . . . . . . . . . . . . . . 58

5.1 Histogram of the dwell time (a) and the distance between the position and the IP (b) for 19 treatment plans including prostate, gynecological and head and neck cases. . . . . . 61

5.2 IP averaged response over 60 time frames for one source dwell position at $22 \mathrm{~cm}$ from the surface of the panel. The dashed rectangles indicate regions where scattering from the frame of the vertically mounted panel or from the table + holders are clearly visible. The arrows show the boundaries of the phantom. The square at the center of the image shows the region $\left(16 \times 16 \mathrm{~cm}^{2}\right)$ around the dwell position considered for fitting purposes. The panel response was corrected only by the dark field signal. . . . . . . . . . . . . 62

5.3 a) pixel sensitivity map obtained using 9 consecutive irradiations performed within a water phantom. b) single line profile (horizontal line around panel position $18 \mathrm{~cm}$ ) through the dwell position, obtained with the source inside a water phantom at $6 \mathrm{~cm}$ from the surface of the detector (IP 2D frame not shown), showing the IP data without correction (Raw) and the results corrected by the pixel sensitivity map. . . . . . . . . . 63

5.4 a) relation between the standard deviation $\left(\sigma_{y}\right)$ of the Gaussian fit described in Equation 4.1 and the distance between the source and the panel (Equation 5.1). The axis at the right shows the residuals between the experimental values and the fit described in Equation 4.1. b) IP response (connected with a line for visualization purposes, Sk(low), showing an increase with time due to the ghosting effect and the z coordinate calculated using the standard deviation $\left(\sigma_{y}\right)$ as described in Equation 5.1 The horizontal dashed line (black) represent the expected $\mathrm{z}$ position $(18 \mathrm{~cm}$ and $22 \mathrm{~cm}) \ldots \ldots \ldots$. . . . . . . 64 
5.5 Source movement in a needle aligned with the IP y axis (equivalent results were obtained using nonaligned needles) for $5 \mathrm{dwell}$ positions with 9.8 seconds programmed dwell time for each position. The distance between the source and the surface of the panel is 21.6 $\mathrm{cm}$. A continuous line connecting the points was added for visualization purposes. . . . . 66

5.6 a) Error between the expected values (treatment plan) and the measured dwell positions. Results are shown for 5 catheters (vertical dashed lines indicate different catheters) with 10 dwell positions each (arrows indicate the first dwell position of each catheter). b) Film measurements for 5 dwell positions with planned inter dwell distances of $0.5 \mathrm{~cm}$. Results are shown for the transfer tube straight and at the same height as the needle and adding a curvature to the transfer tube to mimic the experiment using the gynecological applicator. The overlap between the first and second dwell position is indicated with an

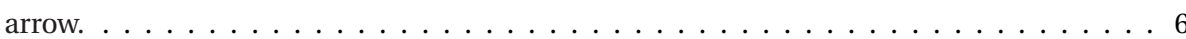

5.7 Dwell times measured for the reference case and Case A. The horizontal dashed line represents the reference dwell time (10.0s) and the continuous horizontal lines define the measurement uncertainties $( \pm 0.2 \mathrm{~s})$. Arrow 1 shows that one dwell position is missing (Case A 1st catheter) whilst arrows 2 and 3 highlight the measurements of the dwell positions for which the dwell times were intentionally modified simulating a treatment error. The vertical dashed lines indicate different catheters. . . . . . . . . . . . 68

5.8 Difference between the dwell positions measured for the reference case and Case A. Differences are shown separately for each axis. The vertical dashed lines indicate different catheters . . . . . . . . . . . . . . . . . . . . . . . .

5.9 Deviation between measured and planned (a) dwell positions (b) and dwell times for a tandem applicator model AL07522002 (Varian Medical Systems). The deviation between measured and planned values was measured 3 times for each dwell position and the error bars correspond to 3 standard deviations. . . . . . . . . . . . . . . . . . . .

5.10 RSD at the applicator's exit surface with a rescaled insert to better evaluate applicators with more than 5 channels (A) and treatment time to deliver 30 Gy with a Sk of $20350 \mathrm{U}$ (5 Ci) source (B) obtained from optimizing applicators with a number of channels ranging from 1 to 20. The reference line in A (horizontal dashed line) for RSD of $2 \%$ represents the arbitrary dose uniformity criterion. The vertical dashed line in B represents the applicators accepted by the same criterion. . . . . . . . . . . . . . . . . . 75

5.11 A Model for the 9-channel HDR contact applicator. A central channel surrounded by a ring of 8 equally distributed channels at $10 \mathrm{~mm}$ radii. B Applicator positioning inside the

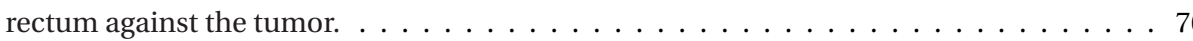

5.12 Comparison between simulated and experimental dose distributions for the 12th irradiation of Table 5.3, at $1 \mathrm{~mm}$ depth. a) isodoses comparing experimental (dotted lines) and simulated (continuous lines) dose distributions; b) simulated dose profile along the line at (a) for $\mathrm{d}_{\text {tip-tip }}=(3.00 \pm 0.15) \mathrm{mm}$, compared to the experimental dose

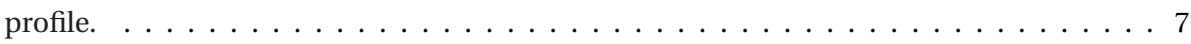


5.13 Simulation of the dose deviation that a source inclination of 2.2 deg would cause in the dose distribution at $1 \mathrm{~mm}$ depth when compared to a source perfectly perpendicular to the treatment surface $\left(\theta_{\mathrm{s}}=0 \mathrm{deg}\right) \ldots \ldots \ldots \ldots \ldots \ldots \ldots$

5.14 Simulated normalized dose at the applicator surface for a region of $40 \mathrm{x} 40 \mathrm{~mm}^{2}$ (a), depth isodoses distribution (b), dose profiles at the applicator surface and at $1 \mathrm{~mm}$ depth in water along the lines 1 (c) and 2 (d) from (a). . . . . . . . . . . . . . .

5.15 Comparison between dose distributions generated by the Papillon 50 device with a 22 mm proctoscope (Croce et. al. [137]) and the HDR applicator. . . . . . . . . . . . . . 80

5.16 Deviation between measured (IrIS) and planned dwell positions after source path optimization, which was performed by changing the source path on the TPS after each measurement until the average deviation between measured and planned dwell positions was minimized. The image on the left shows a projection of the treatment region of the applicator with dummy sources inserted into the channels (with the only purpose of highlighting the channels that were not clearly visible), as well as the optimized channel path, planned and average measured dwell position over 5 measurements (error bars of 2 standard deviations). The plots on the right show the deviation for channels 3 and 5, which presented the lowest and greatest deviations,

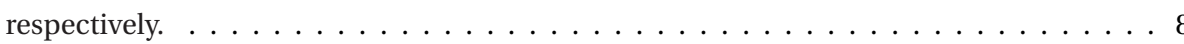

5.17 Isodoses and global deviation (i.e. 100*(Exp-Sim)/Ref. Dose) between measured and simulated dose distribution at depths of $1 \mathrm{~mm}, 2 \mathrm{~mm}$ and $5 \mathrm{~mm}$, adopting reference doses of 28.9, 24.8 and 16.9 Gy, respectively. Each plot has the geometric center of the contact surface adopted as reference axis (represented by vertical and horizontal dashed lines) with the same applicator orientation shown in Figure $5.16 \ldots \ldots$. . . . . . . . 85

5.18 Simulated dose distribution for the MAASTRO applicator with the geometric center of the contact surface adopted as reference axis. a) Dose distribution at $2 \mathrm{~mm}$ depth showing the geometric center of the contact surface (intersection between lines L1 and L2), the center of the dose distribution (intersection between lines L2 and L3) and the $90 \%$ isodose; b) Dose falloff along the center of the dose distribution compared to the dose falloff of the Papillon 50 device with a $22 \mathrm{~mm}$ applicator; c) Dose profiles at $0 \mathrm{~mm}$, $1 \mathrm{~mm}, 2 \mathrm{~mm}$ and $5 \mathrm{~mm}$ depths along lines L2 and L3 and, d) Depth isodoses along lines $\mathrm{L} 2$ and L3. . . . . . . . . . . . . . . . . . . . . . . . . . . 86

5.19 Simulated dose leakage around the proctoscope for a $0.2 \mathrm{~mm}$ thick cylindrical scoring grid. The cylinder with a slanted edge shows how the scoring grid was wrapped around the proctoscope, having the sharp edge of the proctoscope adopted as reference position $(0,0)$ for the scoring grid. The region above the proctoscope edge was masked for better evaluation of dose leakage through the proctoscope wall. . . . . . . . . . . . . 88

5.20 Boxplot showing the deviation from target positions $(90,120$ and $150 \mathrm{~cm}$ ) measured with the CamScale (3 times each) for the source and dummy cables for every odd channel. . 90 
5.21 Deviations between measured and planned absolute dwell positions related to the tip of the transparent QA device. Deviations (grouped by IDD) were obtained by comparison between high-speed camera measurements and the post irradiation report emitted by

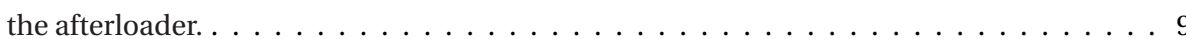

5.22 Difference between planned and measured dwell positions for the ring applicator when distal corrections of 0,1 and $2 \mathrm{~mm}$ are applied to shift the dwell positions and compensate for the smaller IDDs close to the end of the channel. Continuous lines show the deviation for each dwell position while dashed lines show the average deviation over a range of $45 \mathrm{~mm} . \ldots \ldots \ldots$. . . . . . . . . . . . . . . . . . . . . . 97

5.23 Difference in length verification when flexible applicator settings are incorrectly assigned to rigid curved applicators. The red arrows indicate where the dummy cable stops during the length verification when rigid and flexible applicator settings are adopted. 98

5.24 Comparison between measurement and prediction of a source movement for a plan with $5 \mathrm{~mm}$ of interdwell distances and dwell times of 1 second with transit time correction following the Bravos algorithm. The inset shows the first dwell position, with measured dwell time 0.02 seconds lower than planned, causing a time shift on the source movement profile, but with differences between measured and planned dwell times lower than 0.01 seconds for all remaining dwell positions. . . . . . . . . . . . . . . 99

5.25 Dose profiles scored at radial distance of $5 \mathrm{~mm}$ from the source for a plan with 10 dwell positions with IDD of $10 \mathrm{~mm}$ and dwell times of 5 second. Dose profiles were simulated considering the source at each dwell position for its dwell time (no transit - Plan simulation) and considering the source movement following the Bravos and GammaMedplus iX transit time correction algorithms. Dose profiles were compared considering a) absolute dose; b) Absolute deviation from planned dose and c) Relative deviation from planned dose. . . . . . . . . . . . . . . . . . . . . . 100

5.26 Maximum (a) and average (b) dose deviation from planned dose for plans with several IDDs, dwell times and inter-dwell distances considering the source movement with transit time correction according to the algorithms adopted by the Bravos and GammaMedplus iX afterloaders. . . . . . . . . . . . . . . . . . . . . . . . . 102

5.27 Maximum (a) and average (b) dose deviation from planned dose for plans with several IDDs, dwell times and interdwell distances considering the source movement without transit time correction for the Bravos and GammaMedplus iX afterloaders. . . . . . . . . . 103

A.1 a) response profile for two different $\mathrm{Sk}$ ( $\mathrm{Sk}(\mathrm{Low})$ was multiplied by 1.67) with the source, within a water phantom, at $7 \mathrm{~cm}$ (left scale) and $20 \mathrm{~cm}$ (right scale) from the panel. The black arrow shows the region where an abrupt shift was observed. b) ratio between the profiles shown in panel a. c) correction factor as a function of the mean IP response at the boundaries of the response shift. d) corrected profiles $(7 \mathrm{~cm}$ left scale and $20 \mathrm{~cm}$

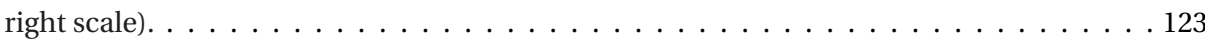


B.1 (a) Experimental setup used to compare film and IP measurements. (b) EBT3 film response; (c) integrated IP response (only for visualization purposes). (d) Comparison between the dwell position. The arrow indicates the dwell position with the largest

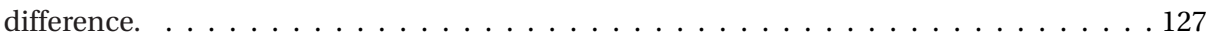





\section{List of Tables}

4.1 Main different features between the GammaMedplus iX and the Bravos systems. . . . . 48

4.2 Summary of experiments performed with the high-speed camera. . . . . . . . . . . 53

5.1 IP response for Sk(Low) (multiplied by 1.67) and Sk(High) for different distances within

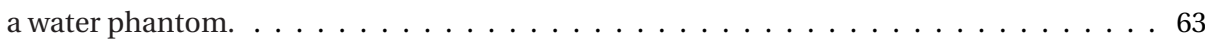

$5.2 \mathrm{z}$ coordinates calculated using Equation 5.1 compared to the reference distance of the source measured using an ultrasonic sensor. The mean value corresponds to an average of 100 time frames \pm one standard deviation ( $1 \sigma$ Type A). The maximum absolute error was obtained evaluating each frame individually corresponding to the largest difference between the $\mathrm{z}$ coordinate and the reference distance. . . . . . . . . . . . . . . . 65

5.3 Gamma analysis (percentage of dose values passing the acceptance criteria) between simulated and experimental data for 9 channel irradiations for scoring planes at 1, 2, 5

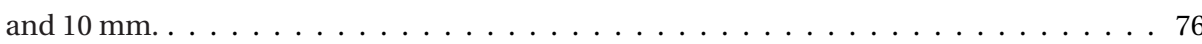

5.4 Deviation between measured and planned absolute dwell positions related to the tip of the transparent QA device for plans with IDD of $5 \mathrm{~mm}$, dwell time of $0.2 \mathrm{sec}$, flexible applicator settings with offset or distal corrections applied. Deviations for the first dwell position are highlighted in a separate column. . . . . . . . . . . . . . . . . . 92

5.5 Minimum dwell time allowed by the Bravos system and measured transit time for the first and remaining dwell positions of plans with IDD ranging from 1 to $100 \mathrm{~mm}$ with first dwell position at the tip of the channel. . . . . . . . . . . . . . . . . . . . 93

5.6 Maximum and average source speed while moving between two consecutive dwell positions inside the QA device for IDD ranging from 1 to $100 \mathrm{~mm}$. Speeds were measured with a high-speed camera and calculated considering a uniformly accelerated movement with $|\mathrm{a}|=(152 \pm 4) \mathrm{cm} / \mathrm{s}^{2} \ldots \ldots \ldots \ldots \ldots \ldots$

5.7 Average deviation between planned and measured dwell positions for the first (most distal) dwell position (results for IDD = 2, 5 and $10 \mathrm{~mm}$ combined) and for all dwell positions for a range of $45 \mathrm{~mm}$ starting at the tip of the applicator for IDDs of 2, 5 and 10 $\mathrm{mm}$ and a range of $20 \mathrm{~mm}$ for IDD of $1 \mathrm{~mm} \ldots \ldots \ldots \ldots$ 
C.1 MC parameters used for the first set of simulations (experimental validation of the

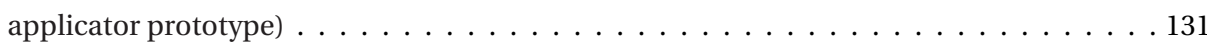

C.2 MC parameters for the second set of simulations (based on the CAD model of the

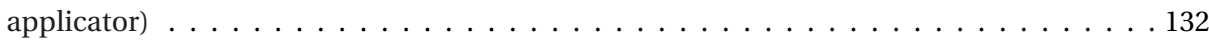

C.3 Uncertainty analysis for the experiments of depth dose measurements and dose leakage measured with EBT-XD films. All uncertainties are expressed by $1 \sigma \ldots \ldots$. . . . . . . . 133 


\section{List of abbreviations}

\begin{tabular}{|c|c|}
\hline AAPM & American Association of Physicists in Medicine \\
\hline BG & Background \\
\hline BT & Brachytherapy \\
\hline CAD & Computer-Aided Design \\
\hline CAX & Central axis \\
\hline CBCT & Cone Beam Computed Tomography \\
\hline CPE & Charged Particle Equilibrium \\
\hline CT & Computed Tomography \\
\hline $\mathrm{CXB}$ & Contact x-ray brachytherapy \\
\hline DTA & Distance to agreement \\
\hline Dw,m & Dose to water in medium \\
\hline EBRT & External Beam Radiotherapy \\
\hline HDR & High dose rate \\
\hline IDD & Inter-dwell distance \\
\hline IMRT & Intensity modulated radiation therapy \\
\hline IP & Imaging Panel \\
\hline IrIS & Iridium Imaging System \\
\hline IVD & In-vivo dosimetry \\
\hline LBTE & Linear Boltzmann Transport Equation \\
\hline LDR & Low Dose Rate \\
\hline
\end{tabular}


LINAC Linear accelerator

MBDCA Model-Based Dose Calculation Algorithm

MC Monte Carlo

MCNP Monte Carlo N-Particle

MRI Magnetic Resonance Imaging

NCS Netherlands Commission on Radiation Dosimetry

OAR Organs at risk

P50 Papillon 50

pCR pathological complete response

PMMA Poly Methyl MethAcrylate

QA Quality Assurance

RSD Relative standard deviation

SBRT Stereotactic body radiation therapy

SLA Stereolithography

TG Task Group

TME Total Mesorectal Excision

TPS Treatment Planning System

TRUS TransRectal UltraSound 
1

Introduction 

Brachytherapy (BT) treatments are employed worldwide to treat a wide variety of cancers such as skin, gynecological, breast, prostate and lung cancer [1]. Brachytherapy has steep dose gradients, offering the possibility to insert the source close or into the tumor. The latter makes the tumor motion to have minor effects for tumor dose since the implant tends to follow its movement. This leads to excellent treatment outcome with less toxicity, better targeting of small treatment regions while sparing organs at risk (OAR) (although the dose delivered to OAR is affected by their motion in relation to the tumor $[2,3])$.

Radiotherapy treatments that include BT have shown outcomes that are as good or even superior than outcomes from external beam radiotherapy (EBRT) techniques without BT for several clinical sites such as prostate, breast, gynecological and skin tumors, among other cancers [4-7]. Moreover, depending on the clinical site, treatments combining BT and EBRT can be more cost-effective than EBRT modalities alone, especially when quality of life of patients is considered $[8,9]$. Nevertheless, studies have shown a decline in BT treatments [5, 10-13] even for sites where BT treatments are highly indicated, such as prostate, cervical and vaginal cancers. The reasons for this decline is mainly due to new EBRT modalities such as stereotactic body radiation therapy (SBRT) and intensity modulated radiation therapy (IMRT), the lack of BT technologies that are already available for EBRT, and shortage of sufficient numbers of trained radiotherapy oncologists in brachytherapy [5]. New BT technologies are in constant development [14], however, the current state of technology and practices in BT still have a great potential of improvement that would yield safer and more accurate treatments.

Figure 1.1 shows an overview of standard practices and alternative methods for BT treatments workflow, highlighting the methods that were used and/or developed for the present thesis.

\section{Standard Treatment workflow and limitations}

A BT treatment workflow changes depending on the oncology center. Nevertheless, it should comprehend the main stages presented in Figure 1.1 (namely afterloader quality assurance (QA), applicator commissioning and QA, treatment planning, delivery and verification) for good practice. The standard practice starts with the afterloader QA, relying mostly on dwell positions verification using radiochromic films. During the applicator commissioning and QA, the applicator geometry is initially verified through computed tomography (CT) or cone beam computed tomography (CBCT) imaging followed by dwell positions verification using radiochromic films $[15,16]$. However, the use of radiochromic films has the limitation of offering only time integrated results, therefore, dwell times are not verified and small interdwell distances (smaller than $5 \mathrm{~mm}$ for a ring applicator using single measurements [17]) cannot be easily evaluated due to the partial overlap of high 


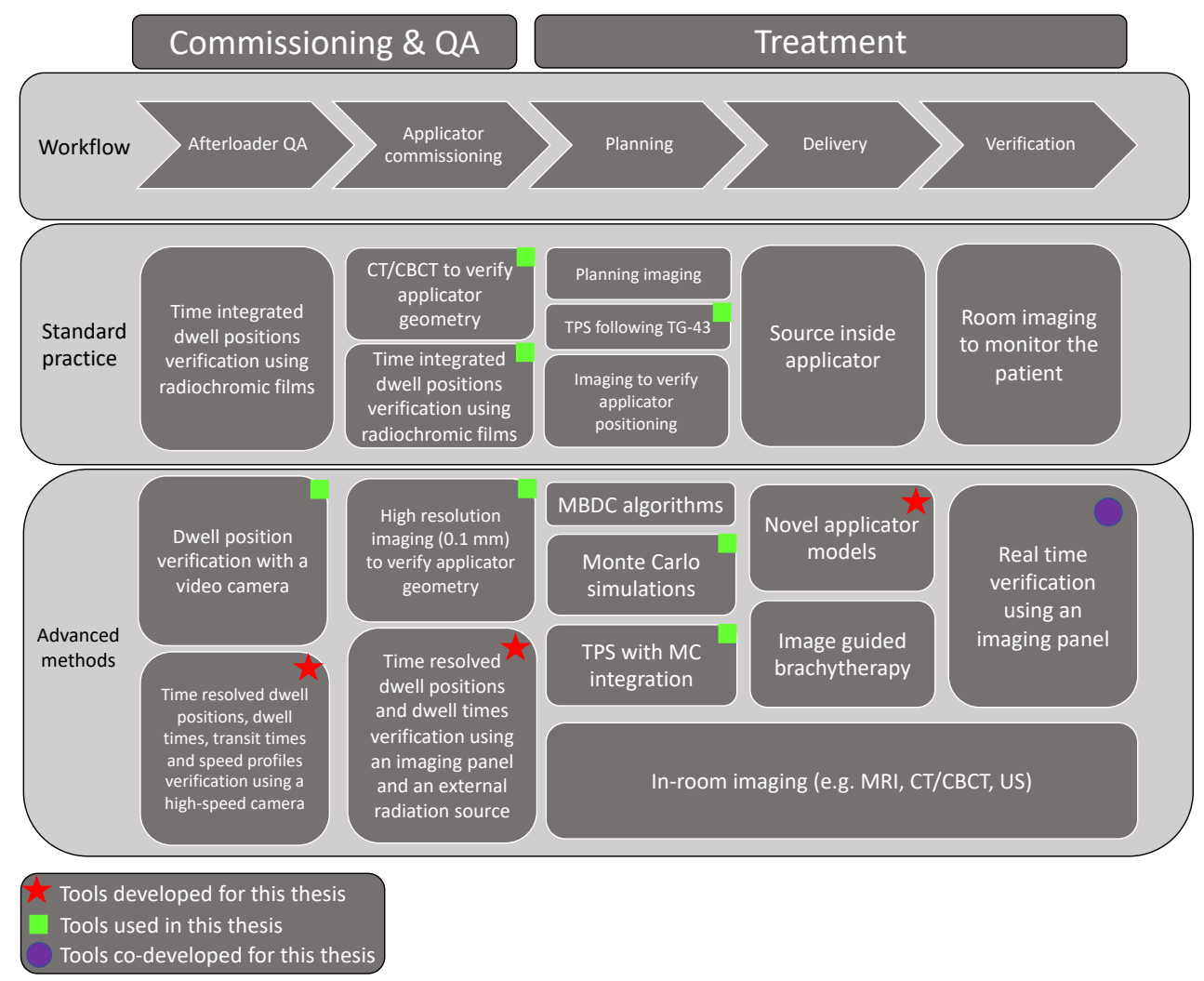

Figure 1.1. Overview of standard practices for BT treatments workflow and most relevant advanced methods for this thesis.

dose regions on the film (marking the dwell positions) despite film's intrinsic high spatial resolution. Furthermore, performing multiple measurements is cumbersome since the film has to be replaced after each irradiation. Exact and reproducible film positioning is also an issue.

The planning stage requires imaging to verify the patient anatomy and correct applicator positioning, which is usually performed in a different room from where the treatment will be delivered, and often with a significant time span in between imaging and treatment. Hence, changes that may occur between the image acquisition and treatment delivery would be unnoticed. Whitaker et. al. [18] has reported a catheter displacement of $5 \mathrm{~mm}$ or more between the planning CT and treatment delivery for $67 \%$ of high dose rate (HDR) prostate BT patients. Moreover, treatment planning systems (TPS) usually adopt the American Association of Physicists in Medicine (AAPM) Task Group report TG43 [19-21] dose calculation formalism by default, which assumes that everything outside the source 
is water and heterogeneities are ignored, both in the applicator and patient. Currently, there is no verification during treatment delivery and errors that might happen during the treatment will be undetected. The lack of treatment verification tools may result in several patients being mistreated until an error is found [22], examples of reported incidents occurred in Netherlands [23] and Canada [24]. Currently, ESTRO has established a Task Group to formulate requirements for in vivo dosimetry verification systems. Each of the aforementioned stages are crucial for an accurate BT treatment, and alternative methods can be adopted to mitigate treatment errors.

\section{Advanced treatment workflow methods}

Afterloader QA can adopt video cameras instead of radiochromic films, allowing more precise dwell positions verification and also dwell times measurements. As an example, Varian Medical Systems (Palo Alto, CA) implemented a camera-based QA device (CamScale) to the Bravos afterloading system released in 2018 [25, 26], which is not time resolved but already allows to easily perform multiple dwell position measurements and system recalibration when necessary (more on this topic in section 4.5.1). Studies have shown that the source cable movement can be measured using regular video cameras [2729] and high speed cameras [25, 30] . Although such techniques are not practical for daily QA, the latter offers a precise and accurate method to evaluate dwell positions, dwell times and source speed profiles, which can be used to evaluate transit time dosimetry.

During the applicator commissioning and QA stages, higher resolution images reduce the uncertainty on the applicator geometry verification. Moreover, the use of an imaging panel (IP) instead of radiochromic films allows time resolved verification of dwell times and dwell positions inside the applicator (see section 2.1).

Another step towards BT treatment improvement is to perform the image acquisition (to verify the applicator positioning) when the patient is already inside the treatment room, reducing the possibility of applicator movement between image acquisition and treatment delivery. While in-room imaging using transrectal ultrasound (TRUS) is already adopted in multiple centers to guide needle implantation for HDR and low dose rate (LDR) prostate treatments in BT [31-33] and has shown good results for cervix treatments [34-36], there are other in-room imaging techniques that can be adopted for brachytherapy, such as CBCT [37], CT-on-rails [38] and Magnetic resonance imaging (MRI) [39].

Treatment planning can take heterogeneities in consideration, as investigated by AAPM Task Group TG-186 [40], by using model-based dose calculation algorithms (MBDCAs) such as collapsed cone convolution [41] and deterministic solution of the linear Boltzmann transport equation (LBTE) [42], Monte Carlo (MC) simulations [43, 44] and 
TPSs with MC integration [45].

Considering heterogeneities in the treatment planning also allows using novel applicator models in which the applicator composition significantly interferes with the resulting dose distribution (e.g. applicators with shielding) [46, 47]. The mentioned in-room imaging techniques can be used to verify the applicator positioning during and after the treatment, however, they do not provide dosimetry information. However, the radiation emitted by the BT source can be detected with an imaging panel (IP) [48-50], providing real time source position verification with the advantage that such technique can also be used for in-vivo dosimetry [48-50]. 
2

Objectives 

This thesis is mainly focused on the development of a novel BT applicator specifically designed for rectal cancer treatments. Nevertheless, to design such applicator, multiple stages of a BT treatment workflow were covered using standard and advanced methods (Figure 1.1) as part of an effort to improve the quality of the applicator modeling, commissioning, QA and dose delivery. The recently released afterloading system Bravos, from Varian Medical Systems (Palo Alto, CA), was also studied to evaluate its treatment delivery limitations. This thesis is also part of a larger project aiming to develop an on-line dosimetry system for brachytherapy patients.

For clarity, this thesis structure was divided into 6 specific objectives (listed below) that are separately explained in the next sections:

1. Evaluate the viability of a pretreatment verification system using imaging panels to track the source movement (section 2.1);

2. Use imaging panels to perform time resolved applicator commissioning (section 2.2);

3. Design, initially as a proof of concept, an applicator capable of generating the dose distribution required for rectal cancer treatments (section 2.3);

4. Design an advanced model of rectal applicator that can be clinically implemented, considering geometry modifications to improve the expected treatment delivery quality, manufacturing limitations, sterilization and materials biocompatibility (section 2.4);

5. Mechanically evaluate a novel afterloader system (Bravos- Varian Medical Systems) to better comprehend treatment delivery limitations (section 2.5);

6. Evaluate the differences in dose distribution resulting from different transit time algorithms between afterloaders (section 2.6).

\subsection{Pretreatment Verification System}

To be successful, a BT treatment has to deliver a dose with an accuracy of a few percent, and with high spatial accuracy. Medical device manufacturers have been developing modern TPSs that are capable of optimizing dose and accounting for human tissue densities, material compositions, body interfaces, body shape and dose perturbations from applicators $[40,51]$. However, an accurate treatment verification method for BT is currently not available [52, 53]. 
In brachytherapy, in particular for HDR treatments, there is an increased need to introduce treatment verification. In the scientific literature, methods have been investigated on measurements of a point dose, or multiple point doses either inside or outside the patient's body [52-55]. This has proven to be cumbersome and, therefore, verification of HDR treatments is not a well-established clinical practice. The dose verification systems employed at most of the hospitals verify only the mechanical operation of the system, and the source activity. Some centers perform patient-specific dosimetry, however there is no method to verify accurately the $3 \mathrm{D}$ treatment dose delivered to the patient. The consequence is that it is very likely that HDR treatment errors occur frequently, which may go undetected. Significant radiation incidents have occurred in brachytherapy treatments [22]. Therefore, a feasible way to verify HDR brachytherapy would be of interest.

Imaging panels (IP) are commonly employed for EBRT using linear accelerators (LINACs). Similar detectors can also be employed in BT treatments as described by Verhaegen et. al [50], Smith et al.[49, 56, 57] and Fonseca et al.[48]. However, significant further developments are necessary before this online dosimetry system becomes available in the clinic with very few papers describing the basic use of this type of panel for brachytherapy and no standard calibration protocol.

This study is part of a larger project aiming to develop an on-line dosimetry system for brachytherapy patients including pre-treatment and in vivo dosimetry. It describes the first step of the project, which consists in developing a system for pre-treatment validation that can be implemented to measure air kerma strength (Sk), source dwell times and source dwell positions before delivering the radiation to the patient. The pre-treatment system described in the manuscript aims to automate part of the measurements, thereby improving accuracy and reducing measurements time. The final goal is to allow the verification of all treatment implants prior to delivery, as recommended by AAPM TG-56 [58], reducing the probability of errors occurring during the treatment. In addition, typical treatment errors were simulated to give a first illustration of which type of errors can be detected by the pretreatment verification system.

\subsection{Iridium Imaging System (IRIS)}

The same principles applied for the pretreatment verification system introduced in section 2.1 can be used to develop a time resolved applicator QA system able to verify dwell positions and dwell times of a BT source inside a clinical applicator and compare them to what was planned, which can also be used for dose verification. Such system (patent pending) was described by Fonseca et al. [17], where a ring applicator (3D Interstitial Ring Applicator, $60^{\circ}$ SET-GM11010190; Varian Medical Systems) was commissioned using an IP, 
model XRD 1640 AG9 ES (Perkin Elmer, Waltham, MA). The system showed promising results and is currently under further development for clinical implementation. The novel QA system was an important tool for the new applicator commissioning and is explained in section 4.2 .

\subsection{Designing A Rectal Applicator For Contact Brachytherapy, A Proof Of Concept}

Worldwide, colorectal cancer is the third most commonly diagnosed cancer in males and the second in females, with 1.65 million new cases and almost 835,000 deaths in 2015 [59]. Nearly one-third of these cancers is located in the rectum [60]. The mainstay of curative rectal cancer treatment involves total mesorectal excision (TME), where the tumor, together with the rectum and surrounding mesorectal fat is removed. TME surgery may result in significant long-term morbidity, such as urgency and fecal incontinence, as well as sexual and urinary dysfunction [61]. Moreover, patients with low seated tumors are often facing resection of the anal complex, resulting in a permanent colostomy [62]. Depending on the anticipated risk of a recurrence, patients may receive neoadjuvant chemoradiotherapy before TME. However, it was observed that there was no visible tumor cells in the resected tissue for $15-27 \%$ of these patients [63]. Recent studies show that for these patients, with pathological complete response (pCR) after chemoradiotherapy, omission of surgery adopting a watch-and-wait regimen has promising outcomes [64-66]. Radiation dose escalation to the tumor appears to increase the chance of pCR and thereby the chance of organ preservation [67]. Dose escalation within the rectum in general leads to increased toxicity and decreased functional outcome, but this can be controlled as long as the treated volumes are kept small [68]. Advantages of endoluminal boosting techniques, such as contact x-ray brachytherapy (CXB) using $50 \mathrm{kV}$ X-rays, include the possibility of better targeting small volumes than EBRT, providing preservation of OAR and better toxicity profiles [69]. Compared to the currently existing BT techniques [7072], favorable characteristics of CXB include: more accurate positioning of the applicator; steeper depth-dose profile and a more selective treatment surface.

CXB techniques have been used over 80 years [73], however, it's use is currently limited to a few centers [74] due to the limited availability of the device until the Papillon 50 (P50) [75] started to be produced by Ariane Medical System (Alfreton, UK) in 2009. Moreover, the lack of planning tools and its high cost hampered the widespread use of the device.

Hence, a novel applicator was designed in this work, as a proof of concept, to demonstrate that it is possible to deliver a dose distribution similar to the one delivered by the P50 device, but with the advantage of using commonly available HDR ${ }^{192} \operatorname{Ir}$ BT sources and 
TPS.

\subsection{Advanced Design Of A Rectal Applicator For Contact Brachytherapy}

The rectal applicator (section 2.3) initially designed as a proof of concept [76] showed promising results as a cost-effective solution for endoluminal rectal boosting with the possibility of TPS integration. The applicator had a cylindrical design with lateral shielding, positioning the source at multiple points perpendicular to the contact surface, and a steep dose falloff could be achieved by placing the sources close to the tumor due to the inverse square law (the applicator is fully described in section 4.4.1). Nonetheless, the uncertainty on source positioning for current available afterloaders $( \pm 1.0 \mathrm{~mm}[77$, 78]) would directly affect the distance between the sources and the tumor, changing the dose distribution and dose falloff shapes. Moreover, having the source perpendicular to the tumor results in a lower dose rate than when the source is parallel and close to the tumor, requiring more lateral shielding to protect OAR. Furthermore, several discussions among experts in the field revealed that a straight edge on the applicator head would be less practical than a slanted edge design, that would simplify positioning the applicator placed through the anal canal on a tumor originating from the side wall. To solve all these problems, the original design was revised aiming for a model that can be implemented in clinical practice.

Therefore, a new applicator prototype (MAASTRO applicator), that has been designed to position the source parallel to the tumor, was modeled, built in close collaboration with a major medical equipment vendor (Varian Medical Systems) and experimentally validated. The MAASTRO applicator provides a higher dose rate with lower dose leakage, and a dose distribution less susceptible to non-uniformity due to source position uncertainties.

\subsection{Mechanical Evaluation Of The Bravos Brachytherapy System}

Bravos is the brachytherapy system released by Varian Medical Systems (Palo Alto, CA) in October of 2018, comprehending an afterloader with 30 channels, a CamScale (a new device that provides tools for daily QA and recalibration for both source and dummy cables) and new control software. The Bravos system uses the same ${ }^{192}$ Ir HDR source as its predecessor, the GammaMedplus iX [79] and it has several new features, such as a reformulated transit time algorithm, differentiation between rigid and flexible applicator settings for any of the channels (an obstruction test or push test is performed for rigid applicators) and a channel length measurement feature. More features are listed in ??.

Since the afterloader and its controlling system play an essential role for precise BT treatment delivery, the Bravos system was evaluated for clinically relevant scenarios, 
verifying its precision and accuracy for parameters such as dwell positions, dwell times and transit time corrections, for a range of applicators, using multiple QA methods.

\subsection{Transit Time}

While creating a BT plan, the TPS ignores the fact that the radioactive source has a limited speed and delivers dose while it is in transit (i.e. moving between dwell positions and moving out of the afterloader and back to it). An approximate correction for this extra dose due to the transit time is applied by the afterloader, reducing the dwell time for each dwell position according to an algorithm that calculates the transit time. Such algorithm is not the same for different afterloader models because the way the source moves (e.g. speed profiles) is not the same either. Moreover, there is no perfect transit time correction algorithm since the time correction is always applied to the dwell position while the transit dose is continuously delivered during the whole source movement.

The transit time correction, however, can have a considerable impact on the dose distribution, especially for HDR treatments [80] with many dwell positions with short dwell times, and most studies aimed to determine the transit time, adopt the average source speed instead of instantaneous source speed, which does not represent a realistic approach for the source movement [27, 28, 81]. Instantaneous source speed was measured by Minamisawa et al. [82] using an optical fiber detector, and the corresponding transit dose was later simulated and experimentally validated by Fonseca et al. [83]. Instantaneous source speed profiles were also evaluated by Fonseca et al. [30] and Bellezzo et al. [25] (see subsection 4.5.2) using a high speed camera.

Since one of the main differences between the GammaMedplus iX and the Bravos afterloaders (both from Varian Medical Systems) is the reformulation of the transit time correction algorithm, the impact that such change has on the dose distribution was evaluated using the instantaneous source speed measured with a high speed camera by Bellezzo et al. [25] and MC simulations of the corresponding dose distribution. 

3

\section{Literature Review}



This section presents a brief literature review regarding the most relevant topics explored in this thesis. In other parts of the thesis more detailed literature overviews can be found. Shortly after the discovery of radioactivity by Becquerel in 1896, the first medical experiences with brachytherapy were reported in the 1900s for treatments using 226Ra [84-86]. However, brachytherapists had to work quickly to reduce personal and support staff exposure to radiation [87, 88]. To overcome this problem of personnel exposure, the remote afterloading system was introduced in the early 1960s by Walstam [89] and Henschke et al.[90, 91], however, without dwell position controlling. One big milestone towards modern brachytherapy techniques was the introduction of the first single-step remote afterloader in the 1970s [1], using small HDR ${ }^{192}$ Ir sources (diameter of $1.1 \mathrm{~mm}$ or less), allowing HDR BT for interstitial techniques. From that point forward, the possibility of individually controlling dwell times and dwell positions allowed patient specific dose optimization, promoting technological advances of more precise and accurate afterloading systems, which are still relevant topics nowadays, when afterloading systems have multiple channels, transit dose correction, precise dwell positions and dwell times [25].

Naturally, the evolution of brachytherapy treatment delivery systems was followed by efforts to better control the resulting dose distribution to the tumor. Initially, when computers were not available and imaging tools were not integrated into brachytherapy procedures, the dose distributions were based on the applicator geometry, but without taking in consideration the patient anatomy and organs at risk [92-94]. With the introduction of 3D image techniques in brachytherapy during the 1970s and 1980s, image guided implants became possible, and the use of transrectal ultrasound (TRUS) (first reported in 1983 [33]) is still adopted for prostate treatments nowadays [95]. Currently, other imaging modalities such as CT and MRI are used for 3D treatment planning [96], and in 2008 the EMBRACE study [97] (endorsed by GEC-ESTRO Gyn) was published, a multicenter study with the purpose to evaluate and benchmark image guided brachytherapy using 3D and 4D MRI for locally advanced cervical cancer.

Several methods to calculate the dose distribution were adopted over the years, summarized by Rivard et al.[92]. Among these methods, the Quimby, Manchester and Paris systems were the most widely adopted for brachytherapy implants.

The Manchester system [98, 99], also known as Paterson and Parker system was designed to maximize the dose homogeneity to the treatment region by using sources with different length and activity in a uniform array, or identical sources in a non-uniform array. Typically, a higher concentration of source strength would be placed close to the tumor edges. The Quimby system $[100,101]$ used sources with the same activity and in a uniform 
spatial distribution to irradiate the tumor. The Paris system [102] was designed to use uniformly spaced ${ }^{192}$ Ir wires with uniform activity distributed in one or two planes. For two plane implants, wires were distributed in equilateral triangle or square geometries. All three aforementioned methods were based on tabulated or nomogram-derived datasets [92].

The current standard for dose calculation in brachytherapy is based on the AAPM TG43 formalism. Initially developed by Nath et al. [103] in 1995 and then updated by Rivard et al.[19] in 2004, the TG-43 formalism adopted dosimetric parameters that depend on the source design and can be directly measured and calculated, resulting in significant improvements in the standardization of dose and dose rate distributions [96]. This formalism is currently adopted as standard by commercial TPSs to calculate dose distributions by the superposition of a single dose distribution in water obtained for a source with cylindrical symmetry.

Although the TG-43 was a milestone to brachytherapy dosimetry standardization, it has the limitation of performing dose calculations in a uniform water medium, not accounting for heterogeneities in the medium and applicator, and its effect on the dose distribution.

Considering TG-43 limitations, another task group (TG-186 [40]) was established in 2012 to review the potential of MBDCAs in brachytherapy for a more precise dose distribution calculation considering tissues and applicators heterogeneities. TPSs from major companies started to have MBDCA capabilities and recommendations for clinical implementation were necessary. Nevertheless, the TG-43 formalism is still the standard for dose calculations in brachytherapy.

Dose calculation formalisms are beyond the scope of this thesis however, it is important to point out that the TG-43 formalism is not able to perform accurate dose calculations for BT applicators that use shielding to help shaping the dose distribution. As an example, Petrokokkinos et al.[46] reported dose deviations of up to $90 \%$ between MC and TG-43 for the shielded side of a shielded cylinder applicator and up to $10 \%$ for the unshielded side, while dose deviations between ACUROS and MC were negligible, except for the penumbra region of the shielding, where deviations between 2 and $10 \%$ were observed.

Although brachytherapy has significantly improved during the last years, it still is a treatment technique susceptible to errors due to multiple steps that are absent or require improvement. The major references to quality assurance in brachytherapy are the AAPM TG-40 [104] and the AAPM TG-56 [58] reports, recommending autoradiographs for pretreatment source position verification, for which the current gold standard consists of using radiochromic films. Such process is not only cumbersome, but the analysis is 
performed in a manual fashion and, despite the intrinsic film high spatial resolution, small inter-dwell distances are hard to distinguish. As an example, the Guidelines for Commissioning Ring Applicators for Use with Bravos Afterloaders (Varian Medical Systems 2018) [105] states that it is difficult to resolve individual dwell positions with IDDs shorter than $10 \mathrm{~mm}$, and that the user should measure the dwell positions by hand or using BrachyVision tools. The NCS report 30 [106] recommends a combination of imaging (xray/CT/MT/US) and autoradiography using films or high resolution detectors to perform QA. A QA system based on imaging panels was successfully developed by Fonseca et al. [17] and further discussed in section 4.2 of this thesis.

Nevertheless, there are no commercial systems available for treatment verification and in-vivo dosimetry (IVD) in brachytherapy. There are several studies using different kind of dosimeters for IVD, such as alanine [107], MOSFETS [108-110] and diodes [111-113], however, the ability of detecting errors with current IVD techniques is highly dependent on the detector position due to steep dose gradients in brachytherapy, and on the detector energy and angular dependence [53]. Due to the lack of commercial systems for treatment verification and IVD, ESTRO has established a Task Group that is currently formulating standards and requirements for IVD for brachytherapy. 

4

Materials and Methods 

This section describes the materials and methods adopted for the study subjects presented in this thesis

\subsection{Pretreatment Verification System}

This section describes the imaging panel calibration performed using a robotic arm to assure high source positioning accuracy, precision and reproducibility. The calibration of an IP includes several steps comprising energy dependence, ghosting effects ${ }^{1}$, angular dependence and pixel sensitivity maps [114, 115]. However, the present study only describes the calibration steps that are necessary for verification of Sk, source positioning and dwell time since the use of this IP for dosimetry will be part of future research.

The calibration conditions were defined based on a range of clinical cases in such a manner that the calibration covers the situations most commonly encountered in the clinic regarding the distance between the source and the panel, acquisition time and spatial resolution.

\subsubsection{Acquisition rate and region of interest}

The panel acquisition rate (frames per second, fps) should be optimal for the dwell time range of the treatment so 10 fps would not be necessary for a treatment with a minimum dwell time of 10 seconds and 1 fps is not enough to verify 0.1 second dwell times that may happen at specific dwell positions. The calibration conditions were defined based on a range of clinical cases, so the calibration covers the situations encountered in the clinic regarding the distance between the source and the panel, acquisition time and spatial resolution.

Treatment plans from 19 patients were evaluated to identify a clinically relevant dwell time range. The plans include 2 prostate cases ( 179 dwell positions), 7 gynecological cases (194 dwell positions) and 10 head and neck cases (175 dwell positions), totaling 548 dwell positions whose dwell times were normalized to an Sk of $40700 \mathrm{U}$. Values were normalized to a high activity so it would be the worst case scenario for the acquisition rate, due to the short dwell times. Therefore, the results were considered only to define the minimum acquisition rate whilst the response of the panel was verified for Sk values comprising the lifespan of the source.

The range of distances between the dwell position and a suitable IP position (assuming the panel will be on the treatment table beneath the patient for in vivo dosimetry or touching the external wall of the water phantom for the pre-treatment verification described in this thesis) were recorded for all the evaluated clinical cases considering the dwell positions

\footnotetext{
${ }^{1}$ Blur caused by a moving object captured by multiple frames
} 
and the position of the patient on the CT table. Some CT images show only part of the patient body without including the CT table so for these cases the distances between the dwell positions and the table are underestimated, since they only take into account the distance between the dwell positions and the bottom edge of the CT image.

In a real clinical case, a carbon fiber frame and a cover would be added between the panel and the patient increasing the distance source-detector by a few centimeters.

\subsubsection{Experimental setup}

Experiments were performed using a GammaMedplus [116] (Varian Medical Systems) ${ }^{192}$ Ir HDR source within a water phantom. Unless stated otherwise, measurements were performed using a phantom with internal volume of $36 \times 30 \times 34 \mathrm{~cm}^{3}$ with $0.8 \mathrm{~cm}$ thick PMMA (Poly Methyl MethAcrylate) wall filled with water.

A robotic arm was used for positioning purposes and was kept as far as possible from the phantom to minimize possible scattering effects. The source-holding needle (plastic needle $\approx 32.5 \mathrm{~cm}$ long, internal diameter $0.16 \mathrm{~cm}$ and external diameter $\approx 0.19 \mathrm{~cm}$ ) was attached to a $\left(0.5 \times 1.0 \times 30 \mathrm{~cm}^{3}\right)$ PMMA slab attached to the robot so the aluminium gripper of the robot arm was not close to the source or even inside of the phantom. The PMMA slab has a higher density $\left(1.19 \mathrm{~g} / \mathrm{cm}^{3}\right)$ than water $\left(1.0 \mathrm{~g} / \mathrm{cm}^{3}\right)$ that can increase the backscattering contribution. However, the small dimensions of the slab and needle compared to the water phantom result in a very small effect that was not considered in this work. Figure 4.1 illustrates the experimental setup highlighting the robotic arm and additional position sensors (Figure 4.1b), described below.
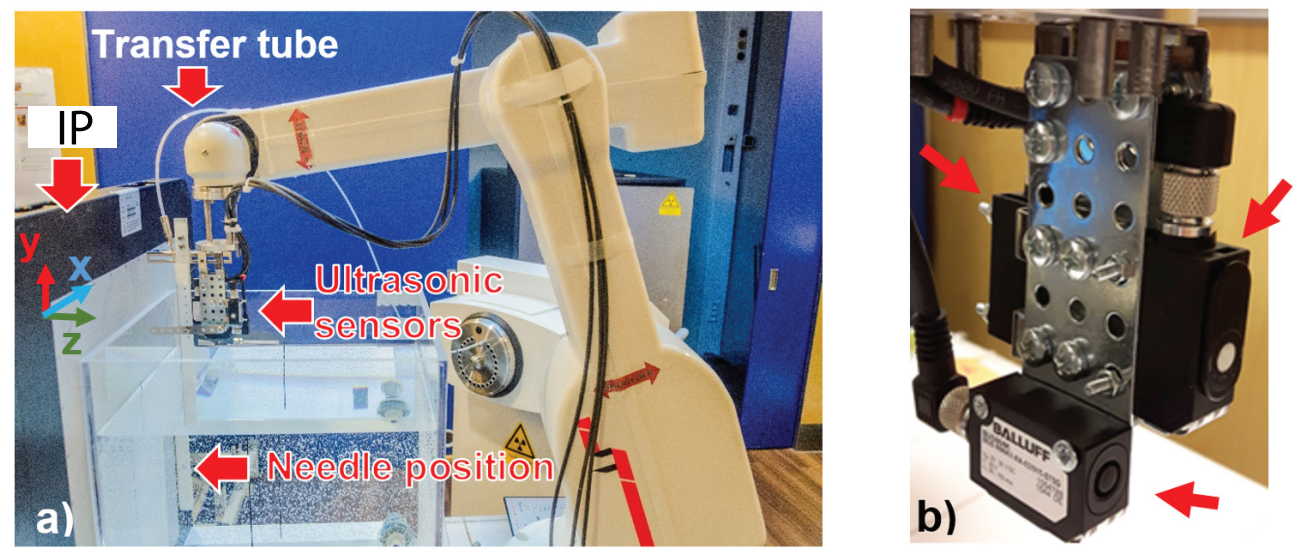

Figure 4.1. a) experimental setup using a robotic arm, a GammaMedplus iX afterloader $\left({ }^{192} \mathrm{Ir}\right.$ source), an IP, ultrasonic sensors and the water phantom employed for most of the experiments; b) detail of the three ultrasonic sensors (arrows) used in the experimental setup. Coordinate system: xy measurement plane; $\mathrm{z}$ distance between the source and the panel. 
Experiments were performed with Sk of $19943 \mathrm{U}$ and $33305 \mathrm{U}$. These SK values are referred to as Sk(low) and Sk(high) in the following. The manufacturer recommends source exchange when it reaches Sk between $17000 \mathrm{U}$ and $13000 \mathrm{U}$ [79], therefore the measurements performed are close to the lowest activities encountered.

\subsubsection{Robotic arm calibration}

The robotic arm (Figure 4.1) R17 (STRobotics, Cambridge, UK) has a reproducibility of about $0.2 \mathrm{~mm}$, according to the manufacturer, depending on the payload (max payload of $2 \mathrm{~kg}$ ) and distance from the origin so the robot goes back to the same position within $0.2 \mathrm{~mm}$. We verified the reproducibility within the water phantom for a payload of about $0.2 \mathrm{~kg}$ (sensors, needle and source support). However, the correlation between the room/phantom and the robot coordinates is not trivial due to a non-linear sag in the several arm joints, varying with the payload and arm position. To correct the sag and account for possible positioning errors the robot was equipped with three ultrasonic sensors (Figure 4.1b), model BUS004K (Balluff, Neuhausen, Germany), with a sensor range between 1.5 and $25 \mathrm{~cm}$ and an uncertainty (Type $\mathrm{A} \pm 1 \sigma$ ) of around $0.5 \%$ of the selected range. Therefore, the absolute uncertainty of the ultrasonic sensors, in centimeters, increases with the range and is approximately $0.025 \mathrm{~cm}$ if an operational range between 5 and $10 \mathrm{~cm}$ is defined. The operational range was defined for each experiment considering the source positions inside the phantom. The sensors provide independent positioning information to correct the robot position using fixed points of reference (e.g. phantom walls).

The robot and the sensors are controlled using an in-house software developed using MatLab, version 8.5 (The MathWorks, Inc., Natick, MA). Therefore, the corrections, if necessary, can be deployed automatically. The reproducibility and accuracy of the system was verified by moving the robot 500 times between 10 distinct points, defined randomly inside of a water phantom, and acquiring the response of the 3 ultrasonic sensors positioned orthogonally.

\subsubsection{Imaging panel (IP)}

The experiments were performed using an IP model XRD 1640 AG9 ES, power source RIDLPX and PCI Framegrabber XRD-FG, (Perkin Elmer, Waltham, MA) with an amorphous silicon sensor operating as a two-dimensional array of $40.96 \times 40.96 \mathrm{~cm}^{2}$ with $1024 \mathrm{x}$ 1024 pixels and pixel size of $0.04 \mathrm{~cm}$. All pixels are behind a sheet of scintillator material which consists of $\mathrm{Gd}_{2} \mathrm{O}_{2} \mathrm{~S}$ :Tb and build-up plates of aluminum and copper. The panel can acquire up to $7.14 \mathrm{fps}$ which is the acquisition rate employed for all the measurements. 


\subsubsection{Response corrections}

This section describes the IP calibration performed using a robotic arm to assure high source positioning accuracy, precision and reproducibility.

The calibration of an IP includes several steps comprising energy dependence, ghosting effects, angular dependence and pixel sensitivity maps [114, 115]. However, this thesis only describes the calibration steps that are necessary for verification of Sk, source positioning and dwell time since the use of this IP for dosimetry will be part of future research.

The panel response needs to be corrected by a dark field offset, pixel sensitivity map, energy dependence and possible non-linearities. These corrections are commonly performed for external photon beams and are based on measurements described in the literature [117-119], however, there is a very limited amount of information on the use of this type of panel for brachytherapy.

Smith et al. [49] performed the calibration of a panel combining an HDR ${ }^{192}$ Ir source and a LINAC (6 MV). We adopted a different approach performing the calibration using only a ${ }^{192}$ Ir source aiming to reduce uncertainties due to energy dependence and to develop a method that doesn't require the availability of a LINAC. This study describes the factors relevant to the treatment verification.

\section{Dark field correction}

The dark field correction subtracts the IP response when there is no radiation, corresponding to a response offset that was subtracted from all the frames acquired [115]. The offset corresponds to the average of 100 frames acquired without radiation.

\section{IP sensitivity map}

The pixel sensitivity varies along the panel and methods to account for this are widely described in the literature. Measurements rely on flood field corrections or by irradiating the panel with the same fluence by displacing the field or the detector [120, 121]. The latter is more convenient since it is a self-sufficient method applicable to EBRT and BT.

The water phantom has limited dimensions so source positions close to the edge of the phantom would have a different scattering contribution than positions close to the center. To ensure equivalent backscattering conditions the coordinates $\mathrm{x}$ (source close to the center of the panel) and $\mathrm{z}$ (source at $6 \mathrm{~cm}$ from the surface of the panel) were kept constant and 9 irradiations were performed with dwell positions $0.2 \mathrm{~cm}$ apart in the y direction. The panel was irradiated 9 times with the source, in water, centered over different points of the panel. The average IP response over 60 time frames was registered using a rigid translation 
function in MatLab to define the shift in y (sy).

Frames were acquired $1 \mathrm{~min}$ after the beginning of the irradiation to minimize possible fluctuations in the panel response and to exclude the effect of the transit dose component. The response of the pixels should be the same if they are at the same position relative to the source in each irradiation. Therefore, the pixel response $R(i, j)$ during the first irradiation should equal to $R(i, j+s y)$ during the second irradiation. Differences can be attributed to the pixel sensitivity, which were obtained for each pixel comparing its response against a reference value $\left(\overline{R_{i j}}\right)$. The reference response of each pixel was defined as the mean of all the pixels receiving the same dose during each one of the 9 irradiations. The pixel sensitivity is then defined as $\overline{R_{i j}}$ divided by its individual pixel $\left(R_{i j}\right)$ response.

\section{Response reproducibility and linearity}

Experiments were performed, with Sk(Low) and Sk(High), for distances between the source and the surface of the detector ranging from 7 to $22 \mathrm{~cm}$. The source was kept at its dwell position until 100 frames were acquired for each of the evaluated distances. The fit described in section 3D Cartesian coordinates was applied to all the frames individually to verify response variations.

The response linearity with Sk was verified keeping the same $\mathrm{z}$ coordinate, in water, for both Sk(Low) and Sk(High) irradiations. Therefore, the intensity was reduced by a factor of 1.67 (due to Sk ratios) whilst the photon spectrum remained the same. The average response of 20 x 20 pixels around the dwell position was compared for both Sk for different distances between the source and the detector.

The response of the panel (average of 100 frames) as a function of the Sk was compared for different distances to verify a possible effect of beam softening on the panel response, due to the photon scattering in the water phantom, since relatively large distances were employed. The irradiations were performed using slightly different $\mathrm{x}$ and $\mathrm{y}$ dwell coordinates so the images obtained using Sk(Low) and Sk(High) were registered to assess individual pixel response (Appendix A) as a function of Sk.

\section{D Cartesian coordinates}

Two dwell position coordinates $\left(x_{0}\right.$ and $y_{0}$ ) can easily be extracted from the 2D IP image whilst the third coordinate ( $\mathrm{z}$ - distance source-panel) can be obtained applying a 2D Gaussian fit (Equation 4.1), whose standard deviation $(\sigma)$ is proportional to the distance between the source and the panel. The relation between $\mathrm{z}$ and $\sigma$ was defined empirically following a similar method described in the literature [49]. The $\sigma$ was divided into two orthogonal components $\sigma_{x}$ and $\sigma_{y}$ to evaluate the effect of the panel reading direction. The hardware reads line by line in the y direction going from the sides to the center [123]. 
Therefore, all the pixels on a line in the y direction where acquired approximately at the same time whilst the pixels in the $\mathrm{x}$ direction were acquired with a very small time delay [123].

$$
\mathrm{F}(\mathrm{x}, \mathrm{y})=\mathrm{A} * \exp \left(-\left(\frac{\left(x-x_{0}\right)^{2}}{2 \sigma_{x}^{2}}\right)-\left(\frac{\left(y-y_{0}\right)^{2}}{2 \sigma_{y}^{2}}\right)\right)
$$

The IP response was acquired every $1 \mathrm{~cm}$ for source/IP distances between 7 and $22 \mathrm{~cm}$ and the experiment was repeated 4 times (moving and repositioning the source after each experiment). The relation between source distance and standard deviation was obtained empirically averaging 100 frames for each position and applying the fit described in Equation 4.1 The data used for the fit consist of the average value obtained using Sk(Low) and Sk(High). The frames were recorded after 1 minute of the beginning of the irradiation to let the panel stabilize. The panel response was normalized for the maximum of each frame, so the amplitude of the peak is equal to 1 to minimize the uncertainty due to variations in the IP response. The coordinates were verified using film measurements for $\mathrm{x}$ and $\mathrm{y}$ (Appendix B. Dwell position verification) and measurements performed using the ultrasonic sensor for $\mathrm{z}$.

Different dwell positions were identified by plotting the measured positions ( $\mathrm{x}, \mathrm{y}$ and z) as a function of the acquisition time. In-house developed software indicates the most probable dwell position based on source coordinates variations using a Laplacian ${ }^{2}$ (summation of second partial derivatives as function of $\mathrm{x}, \mathrm{y}$ and $\mathrm{z}$ ).

\section{Multiple dwell positions and spatial resolution}

Most brachytherapy treatments employ several dwell positions with inter-dwell distances (IDD) ranging from $2 \mathrm{~mm}$ up to a few centimeters depending on the treatment site and the number of needles/catheters. Therefore, it is essential to have at least a $2 \mathrm{~mm}$ spatial resolution to identify different dwell positions. The capability to detect different dwell positions was verified for distances to the panel between 7 and $22 \mathrm{~cm}$ using 5 dwell positions of 9.8 seconds each with IDD of $0.2,0.5$ and $1.0 \mathrm{~cm}$. Dwell position coordinates are identified using the IP response as a function of the time and variations detected using the gradient of the measured source positions. Therefore, variations larger than an interactively pre-defined threshold indicate the movement of the source.

\subsubsection{Clinical application}

A gynecological cylinder applicator with 5 needles (Figure 4.2a) routinely employed in our clinic was selected to simulate possible clinical scenarios for which the IP would be

\footnotetext{
${ }^{2}$ The Laplacian operator was used to highlight when the source is moving
} 
useful to detect mistakes . CT images of the applicator and the phantom were separately acquired, and images were combined using rigid registration. This method simulates a possible clinical case where the applicator is inside the patient, so we artificially added the phantom to define the phantom coordinates related to the CT image of the applicator. As the coordinates of the phantom are known in both the CT image and during the measurements (phantom coordinates related to the IP) it is possible to convert CT to IP coordinates or vice versa.

A treatment plan with 50 dwell positions, 10 positions per needle, $2 \mathrm{~mm}$ inter-dwell distance and 5 seconds dwell time was created as the reference plan. The dwell time in the treatment plan is always defined using a Sk of $40700 \mathrm{U}$ as reference and it is adjusted automatically considering the Sk during the irradiation. Therefore, the dwell time during the experiment was 10.0 seconds after being rescaled $(\mathrm{Sk}=20350 \mathrm{U})$.

The programmed dwell times were used as reference and were verified by recording an independent irradiation (10 dwell positions in a single catheter with $0.5 \mathrm{~cm}$ inter-dwell distance and 10 seconds dwell time in each position).

The robotic arm was used to hold the applicator inside the water phantom (Figure 4.2b) reproducing the coordinates of the CT images. The distances between the applicator and the walls of the phantom were measured using the CT image and reproduced during the experiment.
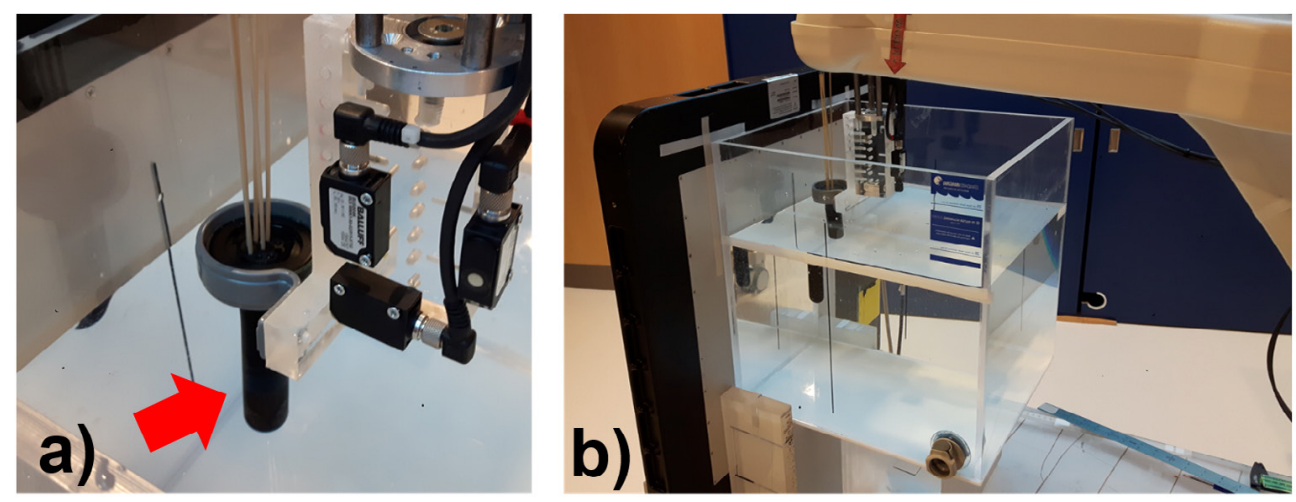

Figure 4.2. Experimental setup used to evaluate clinical scenarios and simulated errors. a) gynecological applicator (red arrow) with 5 needles. b) experimental setup showing the IP, water phantom, gynecological applicator and the robotic arm.

A reference irradiation was performed reproducing the treatment plan. The results were compared using absolute CT coordinates to detect a possible shift of the applicator. The applicator was aligned vertically whilst the transfer guide tube with the catheter connections is in the horizontal position leading to an undesired curvature of the cable 
(the manufacturer states that the transfer tube should be straight during the treatment). The effect of the transfer tube curvature was verified independently using Gafchromic EBT3 films (ISP, Wayne, NJ). A needle with 5 dwell positions (1 second each) was positioned on top of the IP with a film between the panel and the needle. One irradiation with 5 dwell positions, 1 second dwell time at each position and inter dwell distance of $5 \mathrm{~mm}$ was performed with the transfer tube straight and at the same height as the needle. An identical irradiation was performed increasing the curvature of the transfer tube to mimic the experimental condition using the gynecological applicator. It was not possible to use shorter IDDs, as employed in the treatment plan, since the overlap between the dwell position makes the film result blurry and the dwell positions indistinguishable.

Additionally, the treatment plan was modified to simulate treatment errors, and these erroneous plans were delivered:

Case A) the dwell time of the second dwell position of the first needle was set to 0 second, the third dwell time of the second needle was set to 9 seconds and the seventh dwell time of fourth needle was set to 11 seconds;

Case B) an offset was added so the dwell positions were shifted by $10 \mathrm{~mm}$ (first needle), 4 $\mathrm{mm}$ (fourth needle) and $2 \mathrm{~mm}$ (fifth needle);

Case C) The connection of the second and the third needles were exchanged.

The experiments described above simulate potential errors that would affect the treatment and were performed to verify the proposed system. The obtained results simulating erroneous irradiations were compared against the reference irradiation.

In-house developed software was employed to verify the dwell positions, dwell times, number of needles and treatment time. The software is automated and can perform some checks based on the treatment plan information. It verifies the dwell positions and the irradiation sequence to identify a possible misconnection of the transfer tube. The dwell positions are compared against each other to detect the displacement of the needles. In addition, it is possible to measure the position of the tip of the needles since the afterloader employed in this work sends the source to the tip of the needle before stopping at the first dwell position. The dwell times measured using the IP were compared against the planned values for 197 dwell positions of 10 seconds to assess the accuracy of the dwell time measurements.

\subsection{Iridium Imaging System (IrIS)}

This section describes the workflow for applicator commissioning using the IrIS system, showing an example using a tandem applicator (model AL07522002), an IP (PaxScan 2530 
HE Varex, Salt Lake City, UT; which has a maximum acquisition rate of $33 \mathrm{fps}$ ) and a GammaMedplus iX afterloader with a HDR ${ }^{192}$ Ir source. The applicator, IP and afterloader are manufactured by Varian medical systems.

\subsubsection{IrIS working principle}

The working principle for applicator commissioning using the IrIS system was thoroughly described by Fonseca et al. [17], and consists of three main steps:

1. The ${ }^{192}$ Ir source is sent to an imaging channel (Figure 4.3) and the projection of the baseplate with applicator holder (Background (BG) image) is acquired by the IP using the radiation emitted by the source.

2. The applicator is attached to the holder and a second image is acquired using the imaging channel.

3. The source is sent inside the applicator, where time resolved dwell positions are registered by applying a 2D gaussian fit to the IP response (Equation 4.1), and compared to planned dwell positions and dwell times.

\subsubsection{Baseplate and applicator holders}

Figure 4.3 shows each component of applicator commissioning system. The 3D printed baseplate of the IrIS system was designed to be snugly attached to the IP. The vertical distance from the source (inside the imaging channel) to the dwell positions plane inside the applicator once it is attached to the holder is $30 \mathrm{~cm}$. The IP active layer, however, is $3.0 \mathrm{~cm}$ bellow the dwell positions plane, requiring all images to be rescaled by a factor of (30/33 0.9) to represent the real applicator dimensions and dwell positions location (for more details see Fonseca et al. [17]). The baseplate has radiopaque markers (Figure 4.3) with fixed dimensions and positions to verify if the image was properly rescaled and if the source (inside the imaging channel) is in the right position. This will be further discussed in subsection 4.2.4.

The holder shown in Figure 4.3 is for a tandem applicator, and each applicator requires its own holder, which has the applicator code engraved in order to be automatically detected by the IrIS software (feature not yet implemented).

\subsubsection{Treatment Plan Creation}

The treatment plan created for applicator commissioning is based on CT images of the applicator attached to the holder on the baseplate. CT images must include the radiopaque markers shown in Figure 4.3 because they are used to register the CT images 


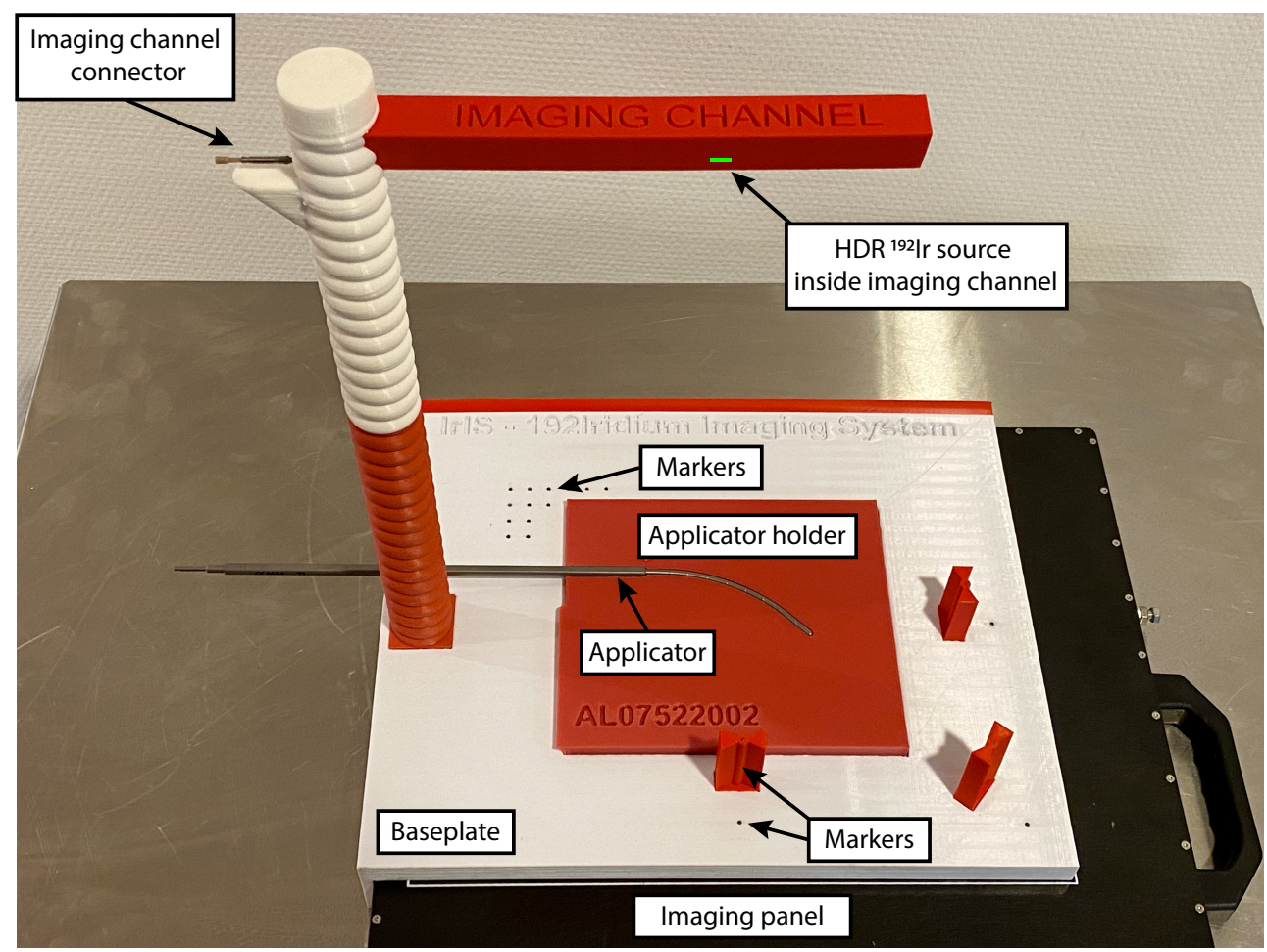

Figure 4.3. Picture of the IrIS system. A 3D printed baseplate with several radiopaque markers used to scale and register images acquired by the IP. Images are acquired using the photons emitted by the HDR ${ }^{192}$ Ir source inside the imaging channel and each applicator has its own 3D printed holder.

(and treatment plan) to the images acquired with the IP. The source path in the treatment plan is originally defined following the center of the applicator and later modified to follow the path experimentally measured to reduce deviations between planned and measured dwell positions.

\subsubsection{IrIS software}

The IrIS software was developed in MATLAB (MathWorks, Massachusetts, US) aiming to automate the measurements as much as possible. Figure 4.4 shows the first verification step, when the BG image (acquired with the IP) was rescaled and the software shows the distance measured (by pixel count) between predefined points, comparing them to the correct dimension (already known). Moreover, the projection of 3 radiopaque columns should be aligned with the measurement lines (Figure 4.4) if the source in the imaging channel is in the right position.

The radiopaque markers are also used to register the treatment plan to the IP images and 


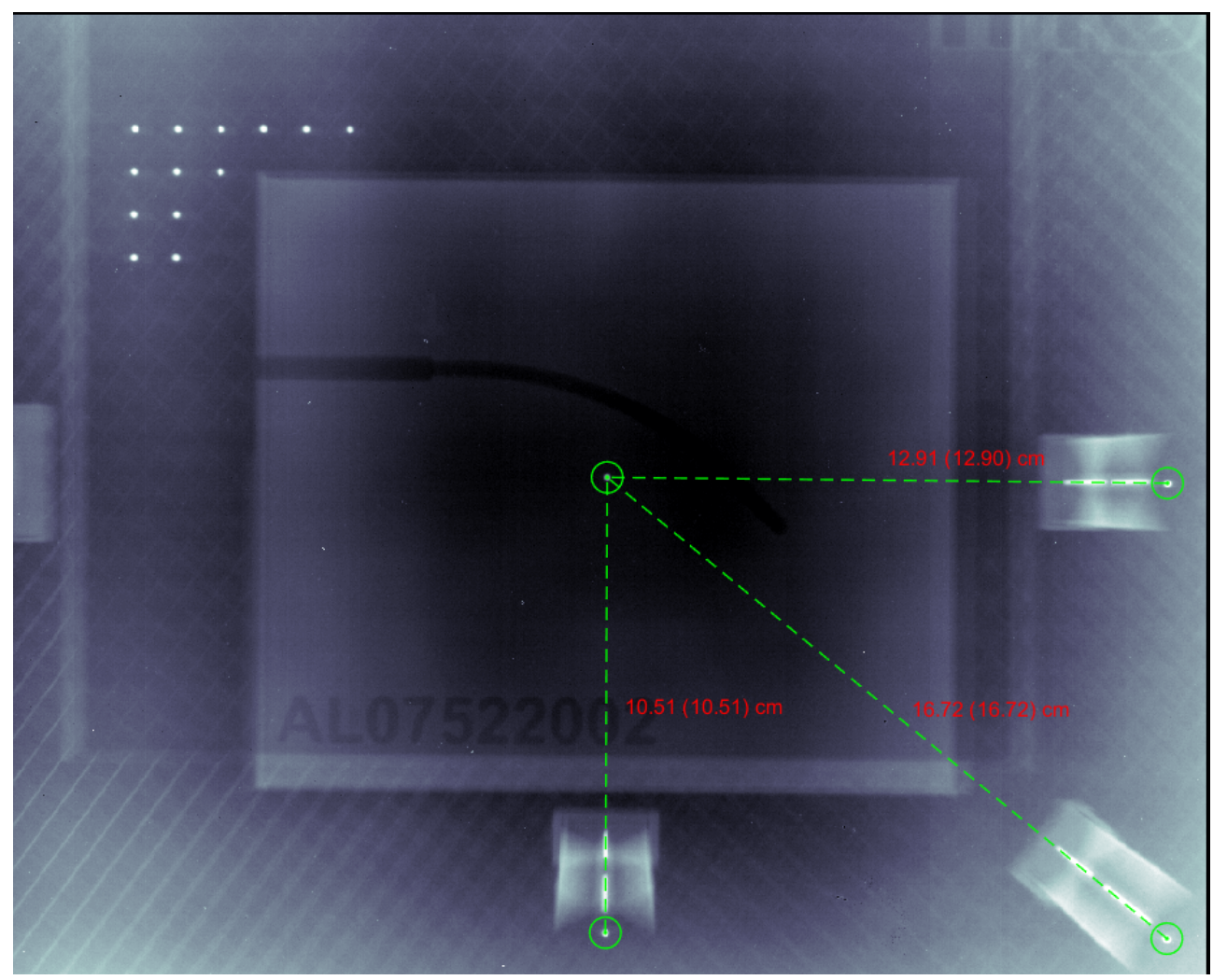

Figure 4.4. Scaled BG image showing measured distances between reference points [measured (expected)], and the projection casted by the vertical markers coincident with the measurement lines (meaning that the BT source is in the correct position inside the imaging channel).

automatically show the planned dwell positions and source path inside the applicator. Figure 4.5 shows the points used for image registration, source path and planned dwell positions, that were automatically defined by the software using rigid registration.

Finally, the treatment plan is delivered and the radiation emitted by the BT source is used to track its position inside the applicator (using Equation 4.1). Figure 4.6a shows the image of the applicator (acquired with the IP and with BG subtracted), planned dwell positions and planned source path, while Figure $4.6 \mathrm{~b}$ shows measured dwell positions on top of planned dwell position. All registrations are automatically performed using the reference points shown in Figure 4.5.

Dwell positions can be measured several times and the IrIS system automatically generates a report with mean deviation between planned and measured dwell positions and dwell times for each dwell position. 


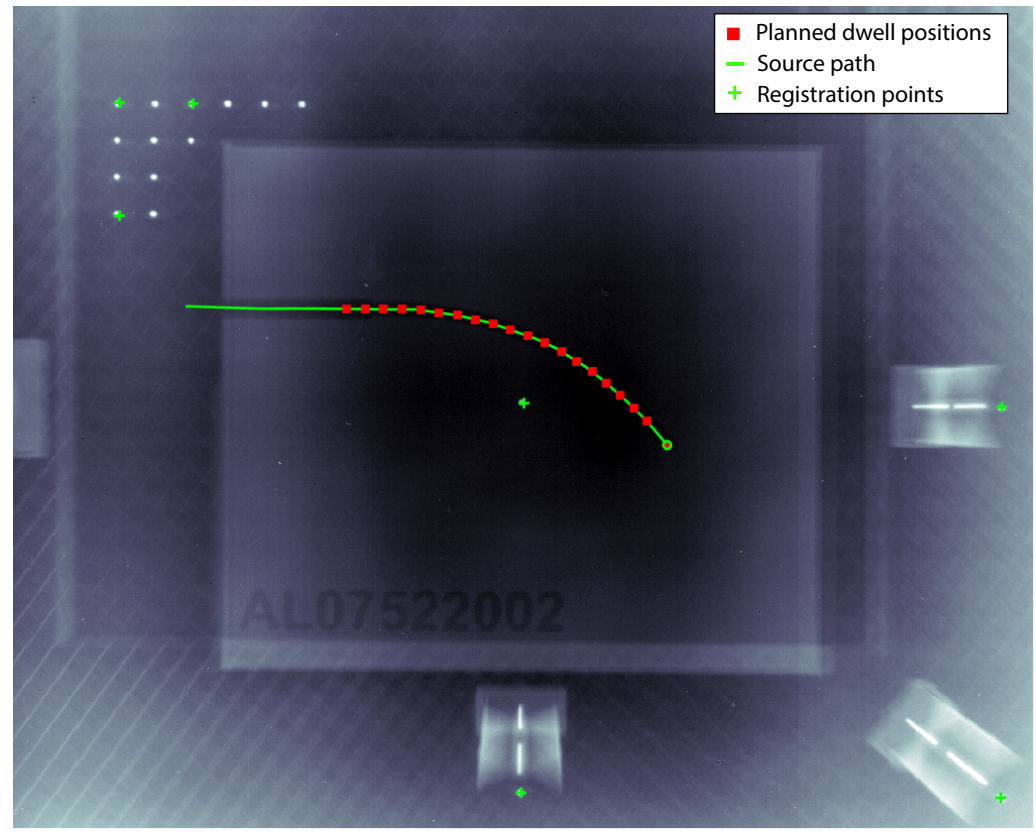

Figure 4.5. Image generated by the IrIS system showing the reference points used for registration, planned source path and planned dwell positions (imported from the TPS).

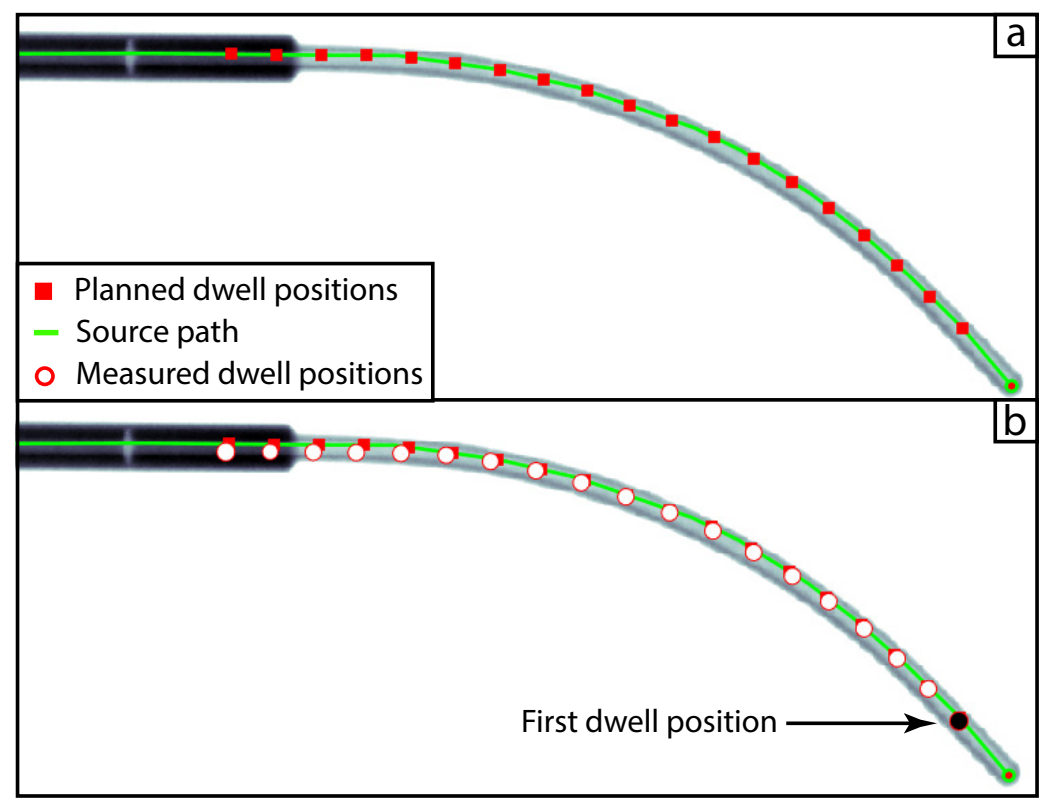

Figure 4.6. Image of the applicator after BG subtraction showing a) Source path and planned dwell positions; b) Measured dwell positions on top of planned dwell positions. 


\subsubsection{Experimental measurements}

Experimental measurements were performed for a tandem applicator model (AL07522002, Varian Medical Systems) with first dwell position having an offset of $5 \mathrm{~mm}$ from the tip, IDD of $5 \mathrm{~mm}$ and dwell times of 1 second (absolute dwell times). The plan had 19 dwell positions and it was executed 3 times.

The IrIS system was also used for commissioning of the advanced model of the rectal applicator in section 4.4 .

\subsection{Designing a rectal applicator for contact brachytherapy, a proof of concept}

This section describes the procedure adopted to design a rectal applicator for contact brachytherapy using the Papillon 50 (P50) as reference regarding dose distribution, applicator dimensions and treatment time.

\subsubsection{Design Of The Applicator}

A HDR BT rectal applicator was designed to be used with a proctoscope for visual guided positioning, using the tip of the applicator as radiation exit surface. Considering a maximum proctoscope diameter of $30 \mathrm{~mm}$ (biggest P50 applicator [75]) and the requirement of having lateral shielding to spare healthy tissues, a cylindrical region with $22 \mathrm{~mm}$ in diameter was defined as the region inside the applicator where the BT sources could be positioned. Within this region, several configurations with a different number of channels and catheter shapes were evaluated with MC simulations to find a configuration that fulfills the clinical requirements (dose distribution, treatment time, applicator dimensions). Given the geometrical restrictions and seeking a high dose rate with a steep dose falloff, a design with multiple straight channels in circular patterns brought in close contact with the tumor was adopted for further investigation and will be presented in this study.

Figure 4.7 shows the initial applicator design, consisting of a $22 \mathrm{~mm}$ diameter PMMA cylindrical core, with $2 \mathrm{~mm}$ pure tungsten $\left(19.25 \mathrm{~g} / \mathrm{cm}^{3}\right)$ lateral shielding and a $1 \mathrm{~mm}$ plastic cap at the exit surface for electron absorption [124, 125] (composition by weight $37.5 \% \mathrm{C}, 3.2 \% \mathrm{H}, 59.3 \% \mathrm{~F}$, with a density of $1.8 \mathrm{~g} / \mathrm{cm}^{3}$, as used in the Leipzig and Valencia applicators).

\subsubsection{Design optimization}

The design of the applicator was optimized by MC simulations using a GammaMedplus HDR ${ }^{192}$ Ir source (Varian Medical Systems) $[116,126,127]$ that was validated against TG43 [103] dose data available in the literature [128] (data not shown) and the consensus 


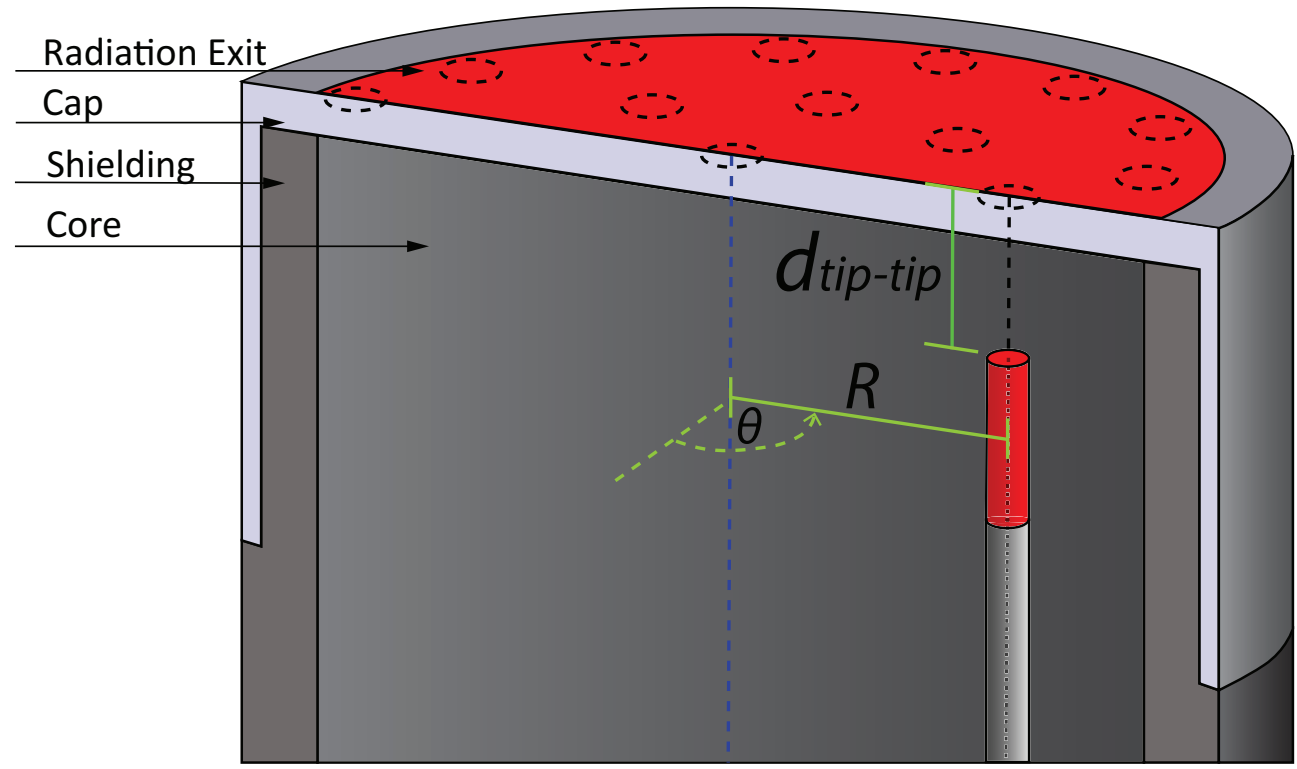

Figure 4.7. Model for the multi-channel HDR contact applicator: A central core holding a number of straight channels positioned in cylindrical patterns surrounded by lateral shielding with a plastic cap on top to stop secondary electrons. The tip of the source $(2.5 \mathrm{~mm}$ from the center) was used as positioning reference because $d_{\text {tip-tip }}$ was easier to measure during experimental validations.

dosimetry data found in the Carleton university database [129]. The MCNP6.1 MC code [130] was used in all simulations of this study. The design was optimized considering the dose distribution falloff, dose uniformity, dose rate and estimated treatment time, using the P50 device as reference.

\section{Dose falloff and dose rate}

The P50 device has a steep dose falloff (30-50\% of the surface dose at $10 \mathrm{~mm}$ depth) and high dose rate $(20-35 \mathrm{~Gy} / \mathrm{min}$ at the treatment surface) where the different values refer to different proctoscope diameters [75, 131]. For the HDR BT applicator, both characteristics are primarily dictated by the distance of the sources to the end surface of the applicator ( $d_{\text {tip-tip }}$, Figure 4.7 ) due to the inverse-square law. The range of $d_{\text {tip-tip }}$ values where the dose falloff from the applicator end surface is encompassed by the ones generated by P50 with different proctoscopes $(22,25$ and $30 \mathrm{~mm})$ was obtained by MC simulations in water. The dose falloff was characterized using $1 \mathrm{~mm}^{3}$ voxels at the applicator central axis (CAX).

\section{Dose distribution uniformity at the radiation exit surface}

Software was developed to calculate the 2D dose distribution at the applicator exit surface by combining MC pre-simulated dose distributions of the model, shown in Figure 4.7, 
to evaluate the relation between dose uniformity and the number of channels in the applicator. For setups containing from 1 to 20 channels, each source could be positioned at $\mathrm{R}=0$ to $10 \mathrm{~mm}$ in $1 \mathrm{~mm}$ steps, $\mathrm{d}_{\text {tip-tip }}$ could range from 1.5 to $3.5 \mathrm{~mm}$ in $1 \mathrm{~mm}$ steps, and $\theta$ from 0 to $360 \mathrm{deg}$ with $1 \mathrm{deg}$ steps. There was no dwell time restriction and the resulting dose rate $(\mathrm{Gy} / \mathrm{min})$ was calculated considering a $40700 \mathrm{U}$ source. The resulting dose distribution uniformity was evaluated considering the relative standard deviation (RSD), defined as the ratio of the standard deviation to the mean, in the scoring region with acceptance criteria of RSD lower than $2 \%$.

For each MC simulation, 1E10 photons were simulated and secondary electrons had their energy deposited locally. The dose was scored in water assuming charged-particle equilibrium (CPE) using a cylindrical grid at the applicator exit surface window with 26 $\mathrm{mm}$ in diameter and $0.1 \mathrm{~mm}$ thick, radial resolution of $0.1 \mathrm{~mm}$ and the angular resolution of 1 degree, resulting in a statistical dose uncertainty lower than $1 \%$ (Type A $\pm 1 \sigma$ ) in all scoring regions.

\section{Treatment time}

The total treatment time consists of two components: the dummy check time and the irradiation time. For the GammaMedplus iX afterloader (Varian Medical Systems), a dummy source is sent twice to the end of each catheter that is going to be used during a treatment to check for obstructions, taking nearly 30 seconds per channel, the second dummy run can be dismissed depending on the system used, but keeping it is a safer clinical practice. The irradiation time is dose rate dependent and will change according to the applicator design and source activity.

Treatments with the P50 device usually deliver fractions of up to $30 \mathrm{~Gy}$ at the tumor surface [132]. The treatment times to deliver the same dose with the different setups of the HDR applicator were evaluated considering a source of $20350 \mathrm{U}$, representing a low activity source, close to a worst-case scenario regarding treatment time.

\subsubsection{Experimental validation}

A simplified prototype of the applicator with 9 straight source channels was built in-house for experimental validation, entirely made of PMMA due to the complexity to in-house manufacture high quality shielding. The transparent PMMA was also used for an in-house QA device, similar to the applicator, but with a ruler inside, enabling to measure $\mathrm{d}_{\text {tip-tip }}$ for the active source by pixel count with a video camera (640x480 pixel resolution) before starting the irradiations and after they were finished to check if there was no position variations during the experiments due to the curvature of a transfer tube [48] and the catheter's diameter size [77]. The same catheter used for the position verification was used 
to irradiate each channel, adopting the same dwell time and dwell position for all of them to better evaluate experimental uncertainties due to transit dose and source position.

These simplifications allowed us to have experimental results with lower uncertainties and validate the MC simulations, however the dose distributions obtained were not intended to be the same as the ones generated by the real applicator during a treatment.

The resulting dose distribution was measured separately 3 times at 1, 2, 5 and $10 \mathrm{~mm}$ depths in a PTW RW3 solid water slab phantom (PTW Freiburg GmbH, Germany) using Gafchromic EBT3 films (ISP, Wayne, NJ), lot 04151402, calibrated between 0.1 and 20 Gy with the triple channel method [133] using a 6 MV photon beam from a Varian TrueBeam accelerator (Varian Medical Systems). Films were scanned 24 hrs after irradiation using an EPSON perfection V750 PRO scanner with 96 dpi resolution.

The dose distribution in the solid water phantom was obtained by simulating the experimental setup using a $0.5 \times 0.5 \times 0.2 \mathrm{~mm}^{3}$ voxel grid with a statistical uncertainty $0.3 \%$ (Type $\mathrm{A} \pm 1 \sigma$ ). A transit dose correction described by Jeong et al. [134] was applied to the simulations. The correction uses the source activity to calculate the equivalent additional dwell time to the irradiation time, for a Sk of $40700 \mathrm{U}$ (10 Ci) source the resulting contribution is $(0.84 \pm 0.09)$ seconds.

Most publications found in the literature that use gamma analysis [135, 136] for radiotherapy adopt acceptance criteria between $3 \mathrm{~mm} / 3 \%$ and $5 \mathrm{~mm} / 5 \%$, TG-119 (IMRT commissioning) adopted $3 \mathrm{~mm} / 3 \%$ for film dosimetry. However, the applicator has a small treatment surface $(20 \mathrm{~mm}$ in diameter) and a distance to agreement (DTA) of $3 \mathrm{~mm}$ or more would hide local dose variations. With the simplified experimental setup and reduced uncertainties, it became possible to adopt more restrictive gamma acceptance criteria, highlighting small deviations between experiments and simulations. The experimental results were compared to the MC simulations using isodoses, dose profiles and gamma analysis with distance to agreement / dose difference acceptance criteria of $1 \mathrm{~mm} / 1 \%$ and $1 \mathrm{~mm} / 3 \%$.

\subsubsection{Source inclination effect}

Since the source may not be perfectly perpendicular to the treatment surface, the effect that a slightly inclined source would cause on the dose distribution was evaluated by experiments where EBT3 films were irradiated at $1 \mathrm{~mm}, 2 \mathrm{~mm}, 5 \mathrm{~mm}$ and $10 \mathrm{~mm}$ depths in solid water using only the central channel of the simplified applicator prototype. Figure 4.8a shows an example of a measured asymmetric dose distribution. A dose profile along the dashed line intercepting the central lower dose region (existing due to the shielding effect of the source tip) with an angle $\phi$, has two peaks (Figure $4.8 \mathrm{~b}$ ), the 
percentage difference between the maxima of each peak $\left(\Delta \mathrm{D}_{(\%)}\right)$ was measured for $\phi$ between 1 and 360 deg and the maximum value of $\Delta \mathrm{D}_{(\%)}\left(\Delta \mathrm{D}_{\% \max }\right)$ was used as a quantifier of the dose asymmetry. With the values of $\Delta \mathrm{D}_{\% \max }$ and the angle $\phi_{\max }$ where it occurs it is possible to determine the source orientation $\left(\theta_{\mathrm{s}}\right.$ and $\phi$ from Figure $\left.4.8 \mathrm{c}\right)$ by comparison with MC simulations of the experiment.

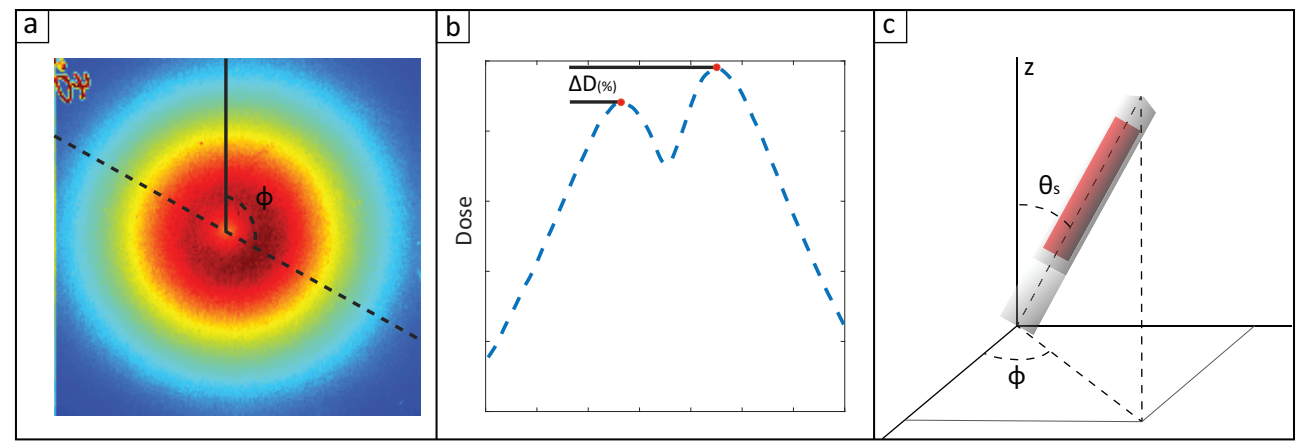

Figure 4.8. a) Example of a film measurement with an asymmetrical dose distribution, irradiated at $10 \mathrm{~mm}$ depth. A dose profile was measured along the dashed line intercepting the lower dose region with an angle $\phi$; b) The resulting dose profile has 2 peaks with a difference between the maxima of $\Delta \mathrm{D}_{(\%)}$. The angle $\phi_{\max }$ is where $\Delta \mathrm{D}_{(\%)}$ is highest $\left(\Delta \mathrm{D}_{\% \max }\right)$; c) With $\phi_{\max }$ and $\Delta \mathrm{D}_{\% \max }$ it is possible to derive the orientation $\theta_{\mathrm{s}}$ and $\phi$ of the source by comparison with MC simulations.

\subsubsection{Virtual shielded model evaluation}

A second MC model of the applicator (including more details such as shielding, plastic cap, optimized channel positions and the $\mathrm{d}_{\text {tip-tip }}$ resulting from the previous section) was built to simulate the $3 \mathrm{D}$ dose distribution in water, which was evaluated considering surface dose distribution, dose profiles at different depths for regions with higher and lower dose uniformity, depth dose profiles and dose falloff. The results were compared with values found in the literature [137] for the P50 device using the $22 \mathrm{~mm}$ proctoscope that generates a similar irradiation area at the treatment surface.

\subsection{Advanced Design Of A Rectal Applicator For Contact Brachytherapy}

This section describes the process of developing an advanced design for the rectal applicator, with the purpose of achieving a model that can be implemented in the clinic. This new applicator received the name of MAASTRO applicator.

The MAASTRO applicator is under development in a partnership between MAASTRO (Maastricht, NL) and Varian Medical Systems, who co-developed the prototype of the applicator and proctoscope for experimental validation. The experimental validation, however, was performed with a simplified model of a proctoscope to reduce experimental 
uncertainties (see section 4.4.3). Finally, a virtual model of the applicator was simulated using MC simulations.

\subsubsection{Design of the MAASTRO applicator}

The MAASTRO applicator was designed to have a dose falloff similar to the one generated by the CXB Papillon 50 device (P50 Ariane Medical Systems, Derbyshire, UK) [137, 138], delivering at least $30 \mathrm{~Gy}$ for a region not smaller than $20 \times 20 \mathrm{~mm}^{2}$ at the applicator contact surface (contact surface shown in Figure 4.9a). This is a typical CXB treatment dose [74, 139], which can be delivered under 9 minutes with the MAASTRO applicator when using a source with air kerma strength of $20375 \mathrm{U}$ (representing a low dose rate, when the source is expected to be replaced), and a field size (90\% isodose) of approximately $20 \times 20 \mathrm{~mm}^{2}$ at $2 \mathrm{~mm}$ depth.

The applicator was designed with a cylindrical shape to be used with a proctoscope that has two main functions: to allow visually guided positioning against rectal wall tumors using its tip as contact surface, and to provide lateral shielding to preserve OAR. Therefore, the MAASTRO applicator has 2 main parts, the proctoscope and the applicator core . The design of the MAASTRO applicator is presented in Figure 4.9. Figure 4.9a shows the channel arrangement inside the applicator with the contact surface highlighted, while Figure $4.9 \mathrm{~b}$ shows a top view of the applicator. Figure $4.9 \mathrm{c}$ shows a cross section (along the dashed line of Figure 4.9b) of the applicator inserted into the proctoscope, and an inset with a perpendicular view of the contact surface.

Both the applicator and the proctoscope have a slanted edge of 50 deg (Figure 4.9c) to allow the channels to bend with a minimum radius of $13 \mathrm{~mm}$ (source cable limitation), placing them parallel to most of the contact surface. The slanted edge also increases the contact surface without increasing the applicator diameter and, it is believed that it may provide a better placement of the applicator against tumors.

Several designs were studied to find a channel arrangement that would result in a homogeneous dose distribution between $1 \mathrm{~mm}$ and $2 \mathrm{~mm}$ depth from the MAASTRO applicator surface. Setups with multiple straight channels following the elliptical surface (similar design as [140]) would have the same problems pointed out in the introduction, and channels emerging from the center and curved towards the applicator edges would create a small treatment region. Hence, the best channel arrangement found to cover the contact surface has 2 elliptical arcs adjoining the edges of the contact surface (outermost channels of Figure 4.9b) and 3 central channels, as presented in Figure 4.9a and b. Such an arrangement places most part of the channels parallel to the contact surface, resulting in a distance of $(2.50 \pm 0.15) \mathrm{mm}$ (uncertainty due to the lumen size) between the center of 


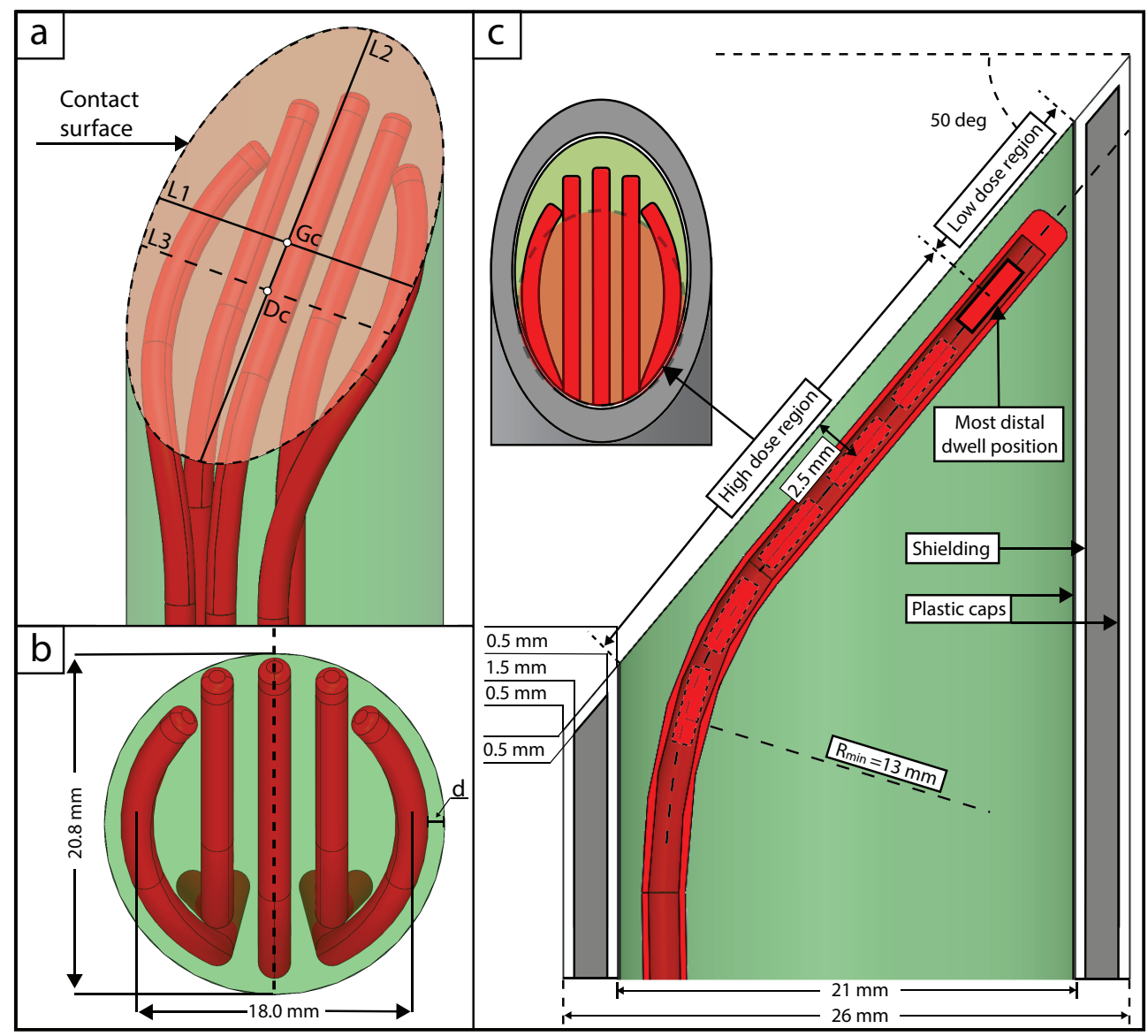

Figure 4.9. Model of the MAASTRO applicator prototype built for experimental validation. a) 3D view of the applicator with the contact surface highlighted. The contact surface geometric center (Gc) is represented by the intersection between lines L1 and L2. However, since the most distal dwell positions of each channel do not reach the edge of the applicator, a shift is anticipated between Gc and the center of the dose distribution (Dc), represented by the intersection between lines L2 and L3; b) Top view of the applicator, showing that there is a minimum distance (d) of approximately $0.5 \mathrm{~mm}$ between the catheter and the lateral wall of the applicator because the channel (not shown) to guide the catheter is slightly thicker than the catheter and there is also a minimum wall thickness that can be 3D printed, resulting in an applicator with $20.8 \mathrm{~mm}$ diameter core with a maximum distance of $18 \mathrm{~mm}$ between the side catheters along the smaller diameter of the contact surface and ; c) Cross section along dashed line on (b) of the applicator inserted into the proctoscope, with a inset showing a perpendicular view of the contact surface, highlighting its high dose region. The proctoscope has a $1.5 \mathrm{~mm}$ thick tungsten alloy (Inermet IT180, $=18.0 \mathrm{~g} / \mathrm{cm}^{3}$ ) shielding encapsulated by $0.5 \mathrm{~mm}$ thick plastic caps. This is done to avoid corrosion of the shielding and stop secondary electrons.

the channel and the contact surface (as shown in Figure 4.9c). This is an advantage when compared to the previous applicator model [140], in which the sources were positioned perpendicular to the contact surface, resulting in a distance uncertainty of $\pm 1.0 \mathrm{~mm}$ due 
to the afterloader precision.

Such an arrangement of the channels, however, does not uniformly cover the contact surface because the most distal source dwell position for each channel does not reach the sharp edge of the applicator, as shown in Figure 4.9c, resulting in a low dose region close to the edge far of the applicator. The high dose region of the contact surface is highlighted in the inset of Figure 4.9c (note that its exact dimensions and shape will change according to the designed treatment plan). Hence, there is an anticipated shift between the geometric center of the contact surface (Gc of Figure 4.9a) and the center of the dose distribution (Dc of Figure 4.9a).

Despite the fact that part of the MAASTRO applicator contact surface delivers a lower dose, the high dose region has approximately the same size as an applicator with a straight edge and sources perpendicular to the contact surface. However, placing the channels parallel to the contact surface provides a greater dose rate, lower dose leakage, and lower uncertainty on the distance between the source and the contact surface.

A disadvantage could be that the low dose region on the contact surface hold a risk of undertreating the target volume. To solve this problem and guide the positioning of the applicator against the tumor considering the high and low dose regions, clearly visible markings will be engraved at the interior of the proctoscope.

\subsubsection{Treatment planning and Monte Carlo simulations}

\subsubsection{Treatment planning}

Two treatment plans were created for experimental validation of the applicator prototype. Plans were created using the TPS BrachyVision v15 (Varian Medical Systems) based on CT images of the applicator with $0.1 \mathrm{~mm}$ resolution acquired with an X-RAD 225Cx system (Precision X-Ray Inc, Connecticut, US). Both plans had interdwell distances of $2 \mathrm{~mm}$ for all the channels, however one plan had fixed dwell times of 1 second for all dwell positions (created to verify dwell positions) while a second plan was optimized to have a dose flatness of $\pm 2 \%$ at $2 \mathrm{~mm}$ depth, resulting in a treatment region (90\% isodose) of an approximately square $20 \times 20 \mathrm{~mm}^{2}$ in water (it is not possible to have a uniform dose distribution at the contact surface due to the proximity to the channels).

All experiments (and CT images acquisition) were performed using the 3D printed applicator core (which was printed in a single piece). However, the proctoscope was printed in multiple parts to encapsulate the shielding. This led to an increase in the geometric uncertainty and didn't allow the applicator contact surface to be flush with the irradiated surface. Therefore, a simplified version of the proctoscope (containing only the shielding piece) was used for experimental validation of the MC simulations. Once 
the MC simulations were validated, a third treatment plan was created based on the CAD model of the applicator and proctoscope to simulate the expected dose distribution for the MAASTRO applicator with the complete proctoscope. The final model of the applicator is expected to be very similar to the simulated CAD model, nonetheless, the applicator will be commissioned before clinical trials.

\subsubsection{Monte Carlo simulations}

MC is currently the gold standard for particle transport simulations. Hence, MC simulations were used as reference for the experimental validation. The MC code MCNP6.1 [130] was used for all simulations in this study, using a model for the GammaMed Plus HDR ${ }^{192}$ Ir source that was previously validated [140] against TG-43 [103] dose data available in the literature [141].

Dose distributions were scored in virtual grids as dose-to-water-in-medium (Dw,m) [142] using track length scoring [143] without electron transport. Electron transport was simulated only to evaluate the dose leakage of the virtual model of the applicator. The MC parameters used for the first set of simulations (experimental validation of the applicator prototype) are summarized in Table C.2 and the MC parameters for the second set of simulations (based on the CAD model of the applicator) are summarized in Table C.3.

\subsubsection{Experimental validation}

The applicator and plastic parts of the proctoscope produced for experimental validation were both 3D printed by Varian Medical Systems using stereolithography (SLA) technology. However, small geometric variations $(0.1-0.2 \mathrm{~mm})$ are inherent of 3D printed models, especially in the case of the proctoscope, that was printed in multiple pieces to encapsulate the tungsten alloy shielding (which was not 3D printed). Hence, the experimental validation of the prototype was performed without the proctoscope to mitigate experimental uncertainties. Only the applicator with a diameter of $20.8 \mathrm{~mm}$ and a $1.5 \mathrm{~mm}$ thick shielding made of tungsten alloy (Inermet IT180 [144], =18.0 g/ $\mathrm{cm}^{3}-95.0 \%$ $\mathrm{W}, 2.5 \% \mathrm{Ni}$ and $2.5 \% \mathrm{Cu}$ ) with internal diameter of $21 \mathrm{~mm}$ were used.

The experimental validation consisted of two main stages: source position verification and film dosimetry.

\subsubsection{Source Position Verification}

Source position verification was performed using the Iridium Imaging System (IrIS patent pending). The working principle for applicator commissioning using the IrIS system was thoroughly described by Fonseca et al. [17]. It uses the radiation emitted by the BT source to acquire a projection of the applicator into an IP and later it sends the source inside the 
applicator and register the dwell positions by applying a 2D gaussian fit to the IP response and compare them to the treatment plan. The IP used by Fonseca et al. had a pixel size of $0.4 \mathrm{~mm}$ and the uncertainty to define the dwell positions was estimated in $0.2 \mathrm{~mm}$. The IP used in the present study was the PaxScan 2530 HE (Varex, Salt Lake City, UT), which has a pixel size of $0.14 \mathrm{~mm}$ and time resolution of up to 0.03 seconds), therefore, the uncertainty to determine the dwell positions is most likely lower than previously reported.

The adopted plan had dwell times of 1 second and inter-dwell distances of $2 \mathrm{~mm}$, with the source trajectory initially defined following the center of each channel. As the results are shown in real-time, it is possible to adjust the treatment plan on the TPS to follow the source trajectory measured experimentally since the source does not follow the center of a curved channel, especially for channels with a small curvature radius as those in the MAASTRO applicator. Once the measured source trajectory matches the trajectory on the TPS, the applicator model on the TPS is properly adjusted and other treatment plans can be adopted. The number of dwell positions per channel ranged from 11 to 14 and the mean deviation from the planned dwell position was averaged over 5 measurements per channel (performed after source path optimization).

\subsubsection{Film Dosimetry}

There were two experimental setups adopted to validate the MC simulations using film dosimetry, one to evaluate the dose distribution at different depths in solid water and a second one to evaluate dose leakage around the shielding. Both setups are shown in Figure 4.10.

Gafchromic EBT-XD films (IPS Technologies, NJ), lot 05081804, were used for both experiments due to theirs extended dose range (0.1 60 Gy [145]), making it suitable to measure depth doses distributions ( 15-30 Gy) and also regions with low dose leakage (less than 2 Gy). Films were calibrated between 0.25 and 50 Gy using the triple channel method [133] with a 6 MV photon beam (EBT-XD films have a low energy dependence [145, 146]) from a Varian TrueBeam accelerator (Varian Medical Systems). Films were placed at the linear accelerator isocenter, between $5 \mathrm{~cm}$ of solid water (on top of the films to avoid the buildup region) and $20 \mathrm{~cm}$ of solid water under the films for backscattering using the PTW RW3 solid water phantom (PTW Freiburg GmbH, Breisgau, DE). Three films were irradiated separately for each dose of the calibration curve and scanned 24 hours after irradiation in portrait mode using an EPSON (Nagano, JP) perfection V750 PRO scanner with 96 dpi resolution.

\section{Depth dose distributions}

The dose distribution was measured at $1 \mathrm{~mm}, 2 \mathrm{~mm}$ and $5 \mathrm{~mm}$ depth in a PTW RW3 solid 
water phantom (PTW Freiburg GmbH, Breisgau, DE). Figure 4.10a shows the experimental setup to measure the dose distribution at different depths. Most dwell positions are 2.5 $\mathrm{mm}$ away from the contact surface (Figure 4.9a), considering the source following the center of the channels), resulting in an intense dose gradient close to the applicator. To reduce experimental uncertainties, three separate films irradiations were performed at each scoring depth, placed flat between $25 \mathrm{~cm}$ of solid water and a top solid water slab of thickness of $1 \mathrm{~mm}, 2 \mathrm{~mm}$ or $5 \mathrm{~mm}$ to define the scoring depth. Films were registered to each other using MATLAB (the MathWorks, Inc., Massachusetts, US), and the average dose distribution of the 3 films at the same depth was compared to the MC simulations that adopted a rectangular virtual grid with $0.2 \times 0.2 \times 0.025$ (film active layer thickness [145]) $\mathrm{mm}^{3}$ voxel size for each scoring depth.

Experimental results were compared to MC simulations using isodoses, global dose ratios [(Measured - Simulated dose)/Ref. dose] and gamma analysis [135, 136] with acceptance criteria of DD/DTA $(3 \% / 1 \mathrm{~mm})$. The reference doses for the isodoses and global dose ratios were obtained from the $\mathrm{MC}$ simulations considering the center of the dose distribution at each depth.

\section{Dose leakage}

Dose leakage was measured with the tip of the applicator centralized inside a $36 \times 30$ x $34 \mathrm{~cm}^{3}$ water phantom model REF 91800 (Standard Imaging, Wisconsin, US) while EBTXD films were wrapped around the shielding at radial distances of $1.0 \mathrm{~mm}$ and 2.0 $\mathrm{mm}$ (Figure 4.10b). Films were held between 2 concentric 3D printed cylinders made of PLA (Polylactic acid, $\left.\rho=1.2 \mathrm{~g} / \mathrm{cm}^{3}-\left(\mathrm{C}_{3} \mathrm{H}_{4} \mathrm{O}_{2}\right)_{\mathrm{n}}\right)$ as illustrated in the inset of Figure $4.10 \mathrm{~b}$, the interior cylinder defined the distance between the films and the shielding $(1.0 \mathrm{~mm}$ or $2.0 \mathrm{~mm}$ ) while the exterior cylinder ( $1 \mathrm{~mm}$ thick) held the film in position. Experimental results were compared to MC simulations that adopted a cylindrical virtual scoring grid with radial resolution of $0.025 \mathrm{~mm}$, axial resolution of $0.5 \mathrm{~mm}$, and angular resolution of 2 deg.

Experimental results were compared to MC simulations using global dose ratios considering the planned dose at $2 \mathrm{~mm}$ depth as the reference dose, and gamma analysis with acceptance criteria of $(3 \% / 1 \mathrm{~mm})$.

\subsubsection{Simulation of the virtual model of the applicator}

The current model of the MAASTRO applicator (and especially the proctoscope), which we experimentally tested in this work, is still undergoing final adjustments considering different materials that would be better for sterilization, more robust and more stable to manufacture. The necessary adjustments in the final design stage aim to minimally 


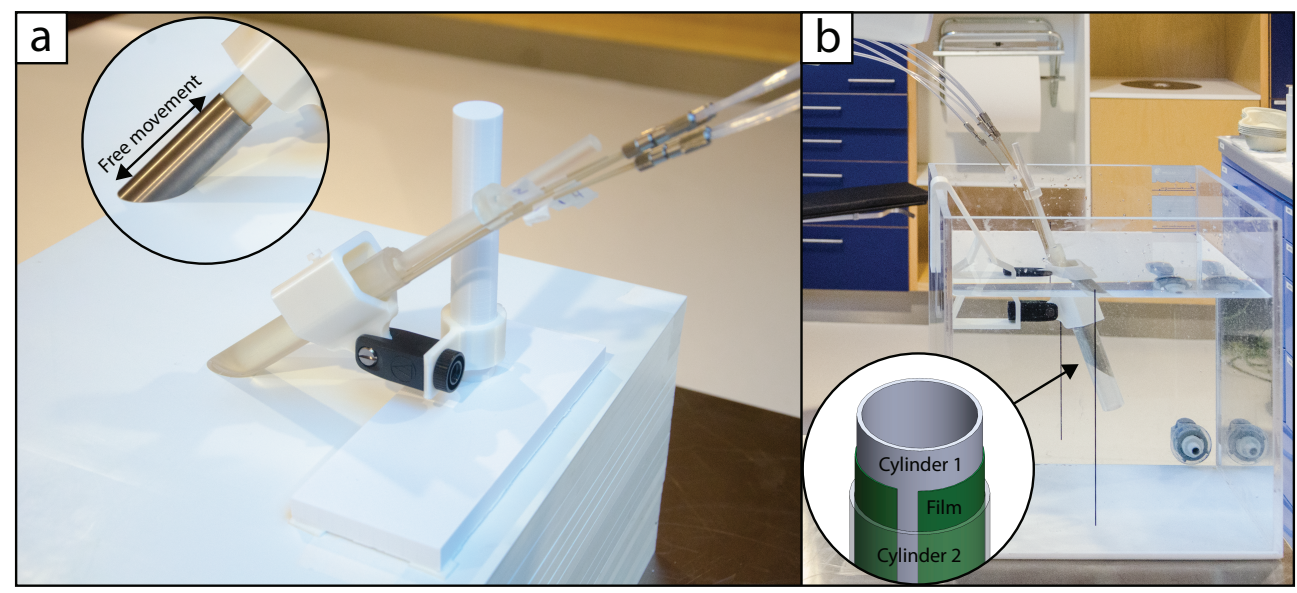

Figure 4.10. a) Experimental setup for dose measurement at different depths in solid water. The applicator was held in position by a 3D printed holder with several deg of freedom to ensure that the contact surface of the applicator was in contact with the solid water surface. Films were placed flat between $25 \mathrm{~cm}$ of solid water and a top solid water slab to define scoring depth. The shielding (inset) was purposely not fixed to the applicator, so it would be possible to shift It along the applicator to verify if the contact surface was flush with the solid water before putting the shielding back in position for irradiation. b) Experiments for dose leakage measurements around the shielding were performed with the applicator axis slightly inclined from the vertical axis ( $\sim 20 \mathrm{deg})$ to reduce transfer guide tubes curvature. The shielding was fixed around the applicator and the film was held between 2 concentric 3D printed cylinders (inset) made of PLA (Polylactic acid, $=1.2 \mathrm{~g} / \mathrm{cm}^{3}$ - $\left.\left(\mathrm{C}_{3} \mathrm{H}_{4} \mathrm{O}_{2}\right)_{n}\right)$, cylinder 1 would snugly fit around the shielding, defining the scoring distance, while the second cylinder would keep the film in place during irradiation.

compromise the dose distribution of the validated model.

In the final design beyond Figure 4.9, instead of plastic, the shielding is encapsulated by a $0.5 \mathrm{~mm}$ layer of stainless steel (ANSI 304), which is the only proctoscope material (apart from the shielding). It was observed experimentally that an applicator with a $20.8 \mathrm{~mm}$ diameter (shown in Figure 4.9) would fit too snugly into a proctoscope with an internal diameter of $21.0 \mathrm{~mm}$. Hence, the diameter of the applicator was reduced to $20.4 \mathrm{~mm}$ to facilitate its insertion into the proctoscope. The applicator is made of PEEK (Polyether ether ketone), which requires a thicker wall ((d) on Figure $4.9 \mathrm{~b})$ for stable manufacturing, bringing the maximum distance between lateral channels (Figure $4.9 \mathrm{~b}$ ) from $18 \mathrm{~mm}$ to 17 $\mathrm{mm}$.

The updated MC model of the applicator was simulated and the dose distribution scored in a rectangular virtual grid with $0.2 \times 0.2 \times 0.1 \mathrm{~mm}^{3}$ voxel size for a region of $60 \times 60 \mathrm{x}$ $5 \mathrm{~mm}^{3}$ to better evaluate dose fluctuations close to the contact surface of the applicator, and in a second grid with $0.5 \mathrm{~mm}^{3}$ voxel size for a region of $60 \times 60 \times 40 \mathrm{~mm}^{3}$ to evaluate depth isodoses and dose falloff. Dose leakage was scored in a cylindrical grid around the 
proctoscope with radial resolution of $0.2 \mathrm{~mm}$, axial resolution of $0.5 \mathrm{~mm}$, and angular resolution of $2 \mathrm{deg}$. The extra dose leakage due to electrons generated at the metallic proctoscope was scored over cylindrical sections positioned in the region with the greatest dose leakage around the proctoscope for a radial distance ranging from $0 \mathrm{~mm}$ to $10 \mathrm{~mm}$ from the proctoscope with radial resolution of $0.2 \mathrm{~mm}$.

\subsection{Mechanical Evaluation Of The Bravos Brachytherapy System}

This section describes the experiments performed to mechanically evaluate the final and commercially available Bravos system. However, no dosimetric tests were performed since the Bravos afterloader uses the GammaMedplus iX source, which is already well documented in the literature [129, 147, 148]. Hence, the afterloader had a source simulation cable instead of an active source, which is identical to a regular source cable (apart from being active), and the focus of this study was the mechanical evaluation of the Bravos system itself. Nevertheless, the source simulation cable is referred to as source.

The main differences between the Bravos and the GammaMedplus iX systems are listed in Table 4.1.

The system evaluation was performed in three groups of experiments:

1. Experiments using the CamScale device: The CamScale was used to verify intra and inter-channel dwelling variability and system calibration by registering both dummy and source cable positions for 3 predefined positions (90, 120 and $150 \mathrm{~cm})$.

2. Source behavior verification inside a QA device: For clinically relevant scenarios in brachytherapy it is important to verify the precision and accuracy for different dwell positions than those verified with the CamScale and evaluate interdwell distances (IDD) and dwell times. To perform these evaluations, the source cable dwelling inside a transparent QA device was recorded with a High-Speed Camera for clinically relevant plans. The High-Speed Camera and the QA device are described in subsection 4.5.2

3. Source behavior verification inside clinical applicators: An external $\mathrm{X}$-ray source and an imaging panel (IP) were used to track the source movement inside clinical applicators. 
Table 4.1. Main different features between the GammaMedplus iX and the Bravos systems.

\begin{tabular}{|c|c|c|}
\hline Feature & GammaMedplus iX & Bravos \\
\hline CamScale device & Absent & Present \\
\hline $\begin{array}{l}\text { Transit time calcula- } \\
\text { tion algorithm (more } \\
\text { details in section } 4.6 \text { ) }\end{array}$ & $\begin{array}{l}\text { Considers only the transit time } \\
\text { between dwell positions }\end{array}$ & $\begin{array}{l}\text { Reformulated to consider the } \\
\text { source movement from the af- } \\
\text { terloader to the most distal po- } \\
\text { sitions }\end{array}$ \\
\hline $\begin{array}{l}\text { Maximum source } \\
\text { speed }\end{array}$ & $63 \mathrm{~cm} / \mathrm{s}$ [79] & $100 \mathrm{~cm} / \mathrm{s}[26]$ \\
\hline $\begin{array}{l}\text { Obstruction verifica- } \\
\text { tion }\end{array}$ & $\begin{array}{l}\text { Performed twice during treat- } \\
\text { ment }\end{array}$ & $\begin{array}{l}\text { Performed once during pre- } \\
\text { treatment and once during } \\
\text { treatment }\end{array}$ \\
\hline Number of channels & 24 & 30 \\
\hline $\begin{array}{l}\text { Possibility of using } \\
\text { coded transfer } \text { guide } \\
\text { tubes }\end{array}$ & Absent & $\begin{array}{l}\text { Channels } 1-3 \text { can be used with } \\
\text { coded transfer guide tubes* }\end{array}$ \\
\hline $\begin{array}{l}\text { Differentiation be- } \\
\text { tween rigid and flexi- } \\
\text { ble applicators (a push } \\
\text { test is performed for } \\
\text { rigid applicators) }\end{array}$ & $\begin{array}{l}\text { Push test is performed for } \\
\text { channels } 1-19 \text { and not for } \\
\text { channels } 20-24\end{array}$ & $\begin{array}{l}\text { The option to perform a push } \\
\text { test or not is defined during } \\
\text { the treatment plan, regardless } \\
\text { of the channel used }\end{array}$ \\
\hline Channel length & Fixed at $130 \mathrm{~cm}$ & From 50 to 1 \\
\hline $\begin{array}{l}\text { Channel length mea- } \\
\text { surement }\end{array}$ & Absent & Present \\
\hline $\begin{array}{l}\text { Afterloader head } \\
\text { height** }\end{array}$ & $\begin{array}{l}\text { Adjustable between } 90 \text { and } 130 \\
\mathrm{~cm}\end{array}$ & Fixe \\
\hline $\begin{array}{l}\text { Distal position correc- } \\
\text { tion }\end{array}$ & $\begin{array}{l}\text { It is possible to add an offset } \\
\text { to the distal position, however, } \\
\text { not to correct it during pre- } \\
\text { treatment }\end{array}$ & $\begin{array}{l}\text { Possible to correct the offset } \\
\text { applied to the distal position } \\
\text { ( } 1 \mathrm{~mm} \text { resolution) during pre- } \\
\text { treatment }\end{array}$ \\
\hline $\begin{array}{l}\text { Display of distal po- } \\
\text { sition when it is at } \\
\text { the end of the channel } \\
(\text { e.g. a channel with } \\
130.0 \mathrm{~cm})\end{array}$ & $\begin{array}{l}\text { There is a } 1 \mathrm{~mm} \text { offset from the } \\
\text { source position to the end of } \\
\text { the channel, however position } \\
\text { is displayed as if there was no } \\
\text { offset, namely } 130.0 \mathrm{~cm}\end{array}$ & $\begin{array}{l}\text { There is a } 1 \mathrm{~mm} \text { offset from } \\
\text { the end of the channel, and the } \\
\text { source position is displayed } \\
\text { including the offset for clarity } \\
\text { sake, namely } 129.9 \mathrm{~cm}\end{array}$ \\
\hline $\begin{array}{l}\text { Pre-treatment check- } \\
\text { list }\end{array}$ & $\begin{array}{l}\text { There is no mandatory pre- } \\
\text { treatment checklist. It is re- } \\
\text { sponsibility of the oncology } \\
\text { center to define an internal } \\
\text { system to guarantee that the } \\
\text { correct treatment is delivered }\end{array}$ & $\begin{array}{l}\text { The treatment can only be } \\
\text { delivered after approving a } \\
\text { checklist with mandatory } \\
\text { fields regarding patient iden- } \\
\text { tity and treatment settings. } \\
\text { Nevertheless, more items can } \\
\text { be added to the checklist }\end{array}$ \\
\hline
\end{tabular}

* Information provided in the user guide but not verified experimentally

** From the floor to the center of the indexer head

\subsubsection{Experiments using the CamScale device}

The CamScale was first introduced as an internal system of the VariSource iX afterloader [149], however, with the only purpose of verifying wire position accuracy. The CamScale 
incorporated into the Bravos system is a separate device intended for daily QA and easy system recalibration when necessary.

The proper distance and alignment of the CamScale with respect to the afterloader is achieved using a built-in laser that places the afterloader and the CamScale at a distance of $50 \pm 1.5 \mathrm{~cm}$ from each other, there is no need of height adjustment. Figure $4.11 \mathrm{a}$ shows the proper CamScale placement with an insert showing the laser projected onto the afterloader surface.

The CamScale contains a calibrated metal ruler with $0.5 \mathrm{~mm}$ resolution and three video cameras (1920 px x 1080 px resolution) that register both dummy and source cable positions at three predefined positions of 90, 120 and $150 \mathrm{~cm}$ (schematic drawing in Figure 4.11b).

Position verification and system recalibration can be performed using any of the afterloader channels, nevertheless, the transfer guide tube presents the lowest curvature when connected to channel 1. For this reason, the user guide [150] recommends using channel 1 for most accurate and consistent results, however such explanation is not mentioned in the current version of the user guide. Moreover, the CamScale can only be used with a specific transfer guide tube and to verify the afterloader calibration, but not to verify length of different transfer guide tubes. Both cables (source and dummy) need to be properly calibrated to ensure that the channel length measured with the dummy cable is suitable for the source cable. Supplementary video $1^{3}$ shows the capture of the Bravos control software screen during the cables position verification of both cables (source and dummy). The system allows the user to perform a recalibration after position verification. Once the CamScale is connected to the afterloader and the system is running, it is required approximately 2 minutes to perform the position verification and system recalibration.

According to the Bravos user guide [150], a system recalibration is suggested if deviations greater than $1 \mathrm{~mm}$ are observed during a CamScale position verification, if the deviation is greater than $2 \mathrm{~mm}$, the calibration is disabled and the user is instructed to contact Varian Customer Support. Three consecutive position verifications using channel 1 were performed before each experiment and, for most accurate results, a system recalibration was performed if deviations greater than $0.3 \mathrm{~mm}$ were reported or if the system was currently calibrated using another channel. The CamScale trolley was never moved between measurements performed in same experimental conditions, however it was moved between different sets of measurements when necessary.

\footnotetext{
${ }^{3}$ Available at: https://doi.org/10.1016/j.brachy.2019.06.005
} 


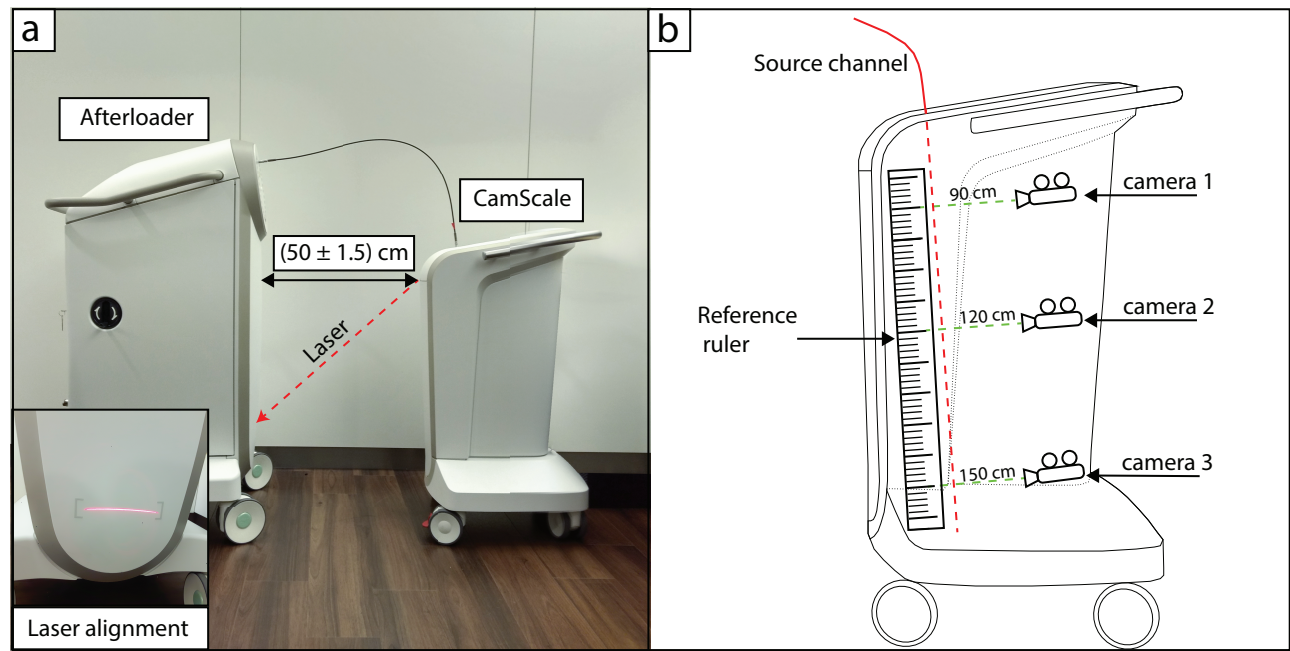

Figure 4.11. a) The ideal distance between the CamScale and the afterloader is $50 \pm 1.5 \mathrm{~cm}$ (measured horizontally at the laser origin height), achieved by laser alignment. The inset shows the laser projected onto the afterloader used for alignment. For a proper alignment the laser projection should be inside the region defined by two markers (visible in the insert figure). b) Schematic drawing showing the position of the cameras and ruler used for position verification in the CamScale.

\subsubsection{Reproducibility and inter-channel variations}

The Bravos afterloader has 30 channels arranged in a circular pattern with a diameter of 19 $\mathrm{cm}$, causing a different transfer guide tube curvature for each channel when connected to the CamScale due to height differences (max. height difference of $18 \mathrm{~cm}$ between channels 1 and 15). The system was calibrated using channel 1 and twenty position verifications were performed using channels 1, 8, 15, 23 and 30 (total of 100 measurements) to evaluate inter and intra-channel variations, followed by 3 measurements for every odd channel to cover a larger number of channels.

It was hypothesized that calibrating the afterloader with the CamScale using one channel and performing a position verification using other channels could affect the position verification results due to the different transfer guide tube curvatures. Channels 1 and 15 were used to evaluate this possible effect since the greatest difference in transfer guide tube curvature occurs between these channels. A total of 60 position verifications were performed using channel 15 while the system was recalibrated before starting the measurements and after every 10 measurements, resulting in a total of 6 recalibrations. The first 3 calibrations were performed using channel 1 and the remaining 3 calibrations using channel 15. 
The Bravos afterloader contains a mechanical system that redirects the source and dummy cables towards the channel that will be used for irradiation (Figure 4.12). To evaluate if the process of redirecting the cables to a different channel affects the system calibration over time, the system was calibrated using channel 1, and 40 CamScale position verifications were performed alternating between channels 1 and 15 (20 measurements per channel). These measurements were repeated in 3 consecutive days for a total of 120 measurements.

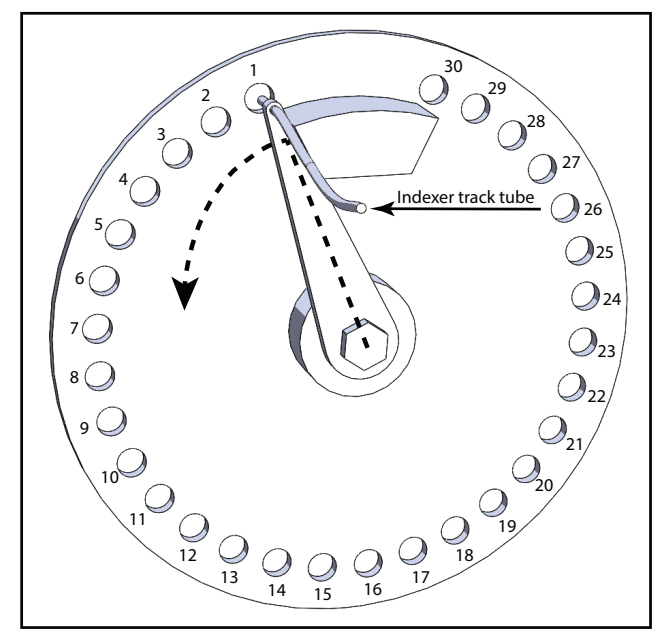

Figure 4.12. Schematic drawing of the mechanism that directs the indexer track tube towards the channel used for irradiation. The indexer track tube is directed to channel 1 by default.

\subsubsection{CamScale behavior when not in reference position}

An accidental CamScale misplacement may occur since there is no interlock between the CamScale and the afterloader. The influence on the source and dummy cable position verification due to a misplacement was investigated for two cases in which the alignment was correct but the distance between the CamScale and the afterloader was off by \pm 9 $\mathrm{cm}$ (maximum distance possible without overextending the transfer guide tube), and for 2 cases in which the alignment was off by $45 \mathrm{deg}$ and $90 \mathrm{deg}$. Five measurements for the channels 1 and 15 were performed for each case.

\subsubsection{Source behavior verification inside a QA device}

Dwell positions, dwell times and transit times were measured with a high-speed camera and compared to the post irradiation report produced by the Bravos control software to verify its precision and accuracy. The post irradiation report shows planned, scaled (considering the source activity) and delivered dwell times for each dwell position. 
Delivered dwell times may be greater than scaled dwell times in cases when scaled dwell times are close to minimum dwell times allowed due to the limited source speed.

\section{Experimental setup}

The experimental setup adopted for the measurements was based on the work of Fonseca et al.[30]. A transparent source step viewer QA device (model GM11008720, Varian Medical Systems) graded to $1 \mathrm{~mm}$ was used to track the source wire dwelling with a high-speed camera (model NEX-FS700R; Sony, Minato, Tokyo, Japan), capable of recording up to 960 frames per second $(\sim 1.04 \mathrm{~ms}$ per frame), offering a high time resolution to verify accurately the smallest dwell time allowed by the afterloader ( 0.1 second) and measure speed profiles and acceleration.

The QA device was evenly illuminated with two diffused light sources positioned one at each side of the camera, that was kept at the same height as the QA device to minimize parallax errors.

The accuracy and precision of the Bravos afterloader regarding dwell positions, dwell times and transit time corrections were evaluated for IDD of 1, 3, 5, 10, 20, 25, 30 and $100 \mathrm{~mm}$, for which the minimum allowed dwell time $\left(\mathrm{t}_{\mathrm{min}}\right)$ is, respectively, $0.1,0.15,0.2$, $0.25,0.30,0.35,0.40$ and 0.40 second. Dwell time precision and accuracy were evaluated for plans adopting dwell times $=t_{\min }$ for each IDD and dwell positions were evaluated for plans with dwell time of 1 second.

The high-speed camera field of view was varied between 30 to $125 \mathrm{~mm}$ according to the IDD of the plan being evaluated, making the pixel size range from 0.04 up to $0.12 \mathrm{~mm}$ due to the resolution of $720 \times 480$ pixels when recording at $960 \mathrm{fps}$, resulting in an uncertainty while tracking the source position ranging from 0.1 to $0.5 \mathrm{~mm}$. All plans were executed using channel 1, adopting the same dwell time and IDD for all dwell positions within the same plan. Unless described otherwise, only the source cable movement was recorded due to the limited buffer size when recording at $960 \mathrm{fps}$ (19 second). Videos were processed with a software developed by the authors in MATLAB (The MathWorks, Inc., Natick, MA) to track the source movement, and transit times were measured following the Bravos Reference Guide [26] (section 4.6.1 shows the difference between calculation methods for transit time by the GammaMedplus iX and the Bravos systems). Average (Vmed) and maximum (Vmax) source speeds were measured for discrete source positions averaged every 0.01 seconds while the source was moving between dwell positions for all IDDs. The source cable acceleration was measured by applying a polynomial curve fitting of second order for the regions where the source was moving between consecutive dwell positions for IDD ranging from 20 to $100 \mathrm{~mm}$ (smaller IDDs didn't have enough points for a good 
curve fitting) or moving from the afterloader to the distal position for all IDDs. Measured values of Vmed and Vmax were compared with its expected values calculated based on the measured acceleration. Table 4.2 shows a summary of the experiments performed with the high-speed camera.

Table 4.2. Summary of experiments performed with the high-speed camera.

\begin{tabular}{|l|l|l|l|l|}
\hline Objectives & IDD(mm) & $\begin{array}{l}\text { Dwell } \\
\text { Time }\end{array}$ & Measurements & Settings \\
\hline $\begin{array}{l}\text { Verify dwell positions and } \\
\text { dwell times }\end{array}$ & $\begin{array}{l}1,3,5,10, \\
20,25,30, \\
100\end{array}$ & $\begin{array}{l}\mathrm{t}_{\mathrm{min}} \\
\text { and } 1 \\
\mathrm{sec}\end{array}$ & 96 & Reference settings* \\
\hline $\begin{array}{l}\text { Verify if the system accurately } \\
\text { reports the delivered dwell } \\
\text { time when transit time correc- } \\
\text { tion is disabled }\end{array}$ & $5,5,10$ & $\mathrm{t}_{\mathrm{min}}$ & 12 & $\begin{array}{l}\text { No transit time } \\
\text { correction }\end{array}$ \\
\hline $\begin{array}{l}\text { Verify correct offset* } \\
\text { Verify correct distal correc- } \\
\text { tion** }\end{array}$ & 5 & $\mathrm{t}_{\min }$ & 15 & $\begin{array}{l}\text { Offset of 0, 1, 2, 4 } \\
\text { and 10 mm }\end{array}$ \\
\hline $\begin{array}{l}\text { Verify difference between flex- } \\
\text { ible and rigid applicator }\end{array}$ & 5 & $\mathrm{t}_{\min }$ & 12 & $\begin{array}{l}\text { Distal correction of } \\
1,2,4 \text { and 10 mm } \\
\text { from tip }\end{array}$ \\
\hline $\begin{array}{l}\text { Compare source and dummy } \\
\text { cables behavior }\end{array}$ & $\begin{array}{l}\text { Single } \\
\text { dwell at } \\
\text { the tip }\end{array}$ & $\mathrm{t}_{\min }$ & 10 & $\begin{array}{l}\text { Rigid applicator } \\
\text { Record dummy } \\
\text { and source }\end{array}$ \\
\hline
\end{tabular}

*The reference settings adopted for the high-speed camera experiments were the standard settings offered by the system when a new plan is created: flexible applicator, transit time correction enabled, first dwell position at the tip of the channel, no distal correction.

**By applying an offset or a distal correction to a channel, all dwell positions will be shifted. The difference is that the offset is defined during the planning process, while the distal correction is during the pre-treatment verification stage, after the channel length measurement is performed.

\subsubsection{Source behavior verification inside clinical applicators}

A kV X-ray source (cone beam CT X-ray unit onboard TrueBeam linear accelerator) and an imaging panel (PaxScan 2530HE, Varex) were used to track the source movement inside 6 clinical applicators: needle (J26-019/GM11007580/2013), ring (VMS R12-061/GM11001240), tandem ring (VMS R11/GM11010990), tandem 15 deg (VMS S09/AL07522000), tandem $45 \mathrm{deg}$ (VMS S09/AL07522002) and Right Colpostat (VMS S09/AL07523000) [151].

The imaging panel was positioned on top of the LINAC treatment table and the applicators were aligned with the surface of the imaging panel. Transfer guide tubes were positioned to avoid excessive curvature. In addition, a thin lead sheet was placed in front of the afterloader to prevent the $\mathrm{X}$-rays coming from the $\mathrm{kV}$ imaging source to trigger the 
afterloader radiation detector and shutdown the system.

The imaging panel acquisition rate was set to $14.8 \mathrm{fps}$ (to synchronize with the X-ray source duty cycle) with spatial resolution of $0.14 \mathrm{~mm}$. The tube voltage was defined at $120 \mathrm{kVp}$ to optimize contrast.

Reference treatment plans were created using the TPS BrachyVision v15 (Varian Medical Systems) based on CT images with $0.1 \mathrm{~mm}$ resolution of the applicators acquired with an X-RAD 225Cx system (Precision X-Ray Inc, Connecticut, US). The source trajectory for each applicator was initially defined following the center of the channel and later adjusted to follow a more realistic path based on the experimental data. CT and imaging panel images were registered considering the geometry of the applicators and user defined landmarks to compare measured and planned dwell positions with an in-house software written in MatLab version 8.5 (The MathWorks, Inc., Natick, MA). The TPS uses the center of the source as reference for the dwell positions whilst the tip of the source is easier to detect using the described experimental setup. Therefore, dwell positions from the treatment plan were shifted by $2.47 \mathrm{~mm}$ along the source trajectory (based on the source geometry found in the literature $[126,152])$ to correspond to the tip of the source when the positions were compared.

Dwell position precision and accuracy were evaluated for plans with rigid applicators settings and IDDs of 1, 2, 5 and $10 \mathrm{~mm}$. Offsets of $0,1,2$ and $5 \mathrm{~mm}$ were applied to the plans with IDD of $5 \mathrm{~mm}$ to evaluate if the average deviation between planned and measured dwell positions could be improved by applying an offset or distal correction to the dwell positions. The difference between rigid and flexible applicator settings was evaluated for plans with IDD of $5 \mathrm{~mm}$ and no offset. Each plan was repeated 5 times, with dwell times of 1 second and dwell positions covering a range of $45 \mathrm{~mm}$ starting at the tip of each applicator for IDDs of 2, 5 and $10 \mathrm{~mm}$ and a range of $20 \mathrm{~mm}$ for IDDs of $1 \mathrm{~mm}$ to better evaluate the source behavior close to the tip of the applicators. The same dwell positions range was adopted for all applicators for consistency while evaluating the Bravos system behavior. Different dwell times were evaluated with the high-speed camera using the QA device due to its higher temporal resolution. A total of 35 plans were executed per applicator. Supplementary video $3^{4}$ shows the source movement inside the tandem $45 \mathrm{deg}$ applicator.

\subsection{Transit Time}

This section describes the materials and methods adopted to evaluate the effect that the new transit time correction algorithm adopted by the Bravos has on the source movement

\footnotetext{
${ }^{4}$ Available at: https://doi.org/10.1016/j.brachy.2019.06.005
} 
and dwell times.

\subsubsection{Differences in dwell time calculation between the GammaMedplus iX and Bravos systems}

As mentioned in section 2.6, the TPS does not account for the extra dose that will be given to each dwell position while the active source is moving. This transit time component is calculated by the afterloader and dwell times are corrected aiming to deliver a dose as close as possible to planned.

The algorithm for transit time correction used by the GammaMedplus iX system was reformulated for the Bravos system.

\section{Transit time correction in GammaMedplus iX system}

For the GammaMedplus iX system, the dwell time (time in dwell position + transit time) is defined as the time between the source leaving the previous dwell position until leaving the current dwell position.

Figure 4.13a shows the position over time for a source approaching the most distal position, and then having 4 dwell positions $\left(D_{w} 1-4\right)$ while being retracted back to the afterloader. According to the algorithm used by the GammaMedplus iX system, the time intervals $\mathrm{T}_{\mathrm{G}} 1-4$ corresponds to the dwell times for dwell positions $\mathrm{D}_{\mathrm{w}} 1-4$, respectively.

However, this algorithm doesn't consider a transit time component for the first and last dwell positions or the transit time when the source passes over the dwell positions while moving to the most distal position.

\section{Transit time correction in Bravos system}

Figure $4.13 \mathrm{~b}$ shows the same dwelling scheme of Figure 4.13a, however with the transit time correction algorithm adopted by the Bravos system. For the Bravos system, the dwell time is considered as the time that the source spends within a region $\left(\mathrm{R}_{\mathrm{i}}\right)$ close to the dwell position $\left(\mathrm{D}_{\mathrm{w}} \mathrm{i}\right)$, including when the source is moving from the afterloader to the most distal position. This region is encompassed by the points $P_{i-1}$ and $P_{i}$, defined respectively as the half distance between the current dwell position $\left(D_{w} i\right)$ and the previous one $\left(D_{w} i-1\right)$, and the half distance between $D_{w} i$ and the next one $\left(D_{w} i+1\right)$ for a maximum half distance of $1.5 \mathrm{~cm}$, which is also adopted as transit time region for single dwell positions.

For the most distal dwell position $\left(D_{w} 1\right)$, the region $\left(R_{1}\right)$ that is accounted for in the dwell time is where the source has a position more distal than $\mathrm{P}_{1}$, making the dwell time equal to the time interval $T_{B} 1$. The region used to calculate the dwell time for $D_{w} 2$ is $R_{2}$, encompassed by $\mathrm{P}_{1}$ and $\mathrm{P}_{2}$, making the dwell time equal to the sum of the time intervals $\mathrm{T}_{\mathrm{B}} 2+\mathrm{T}_{\mathrm{t}} 2, \mathrm{P}_{2}$ and $\mathrm{P}_{3}$ define the region $\mathrm{R}_{3}$ to calculate the dwell time for dwell position Dw3, 


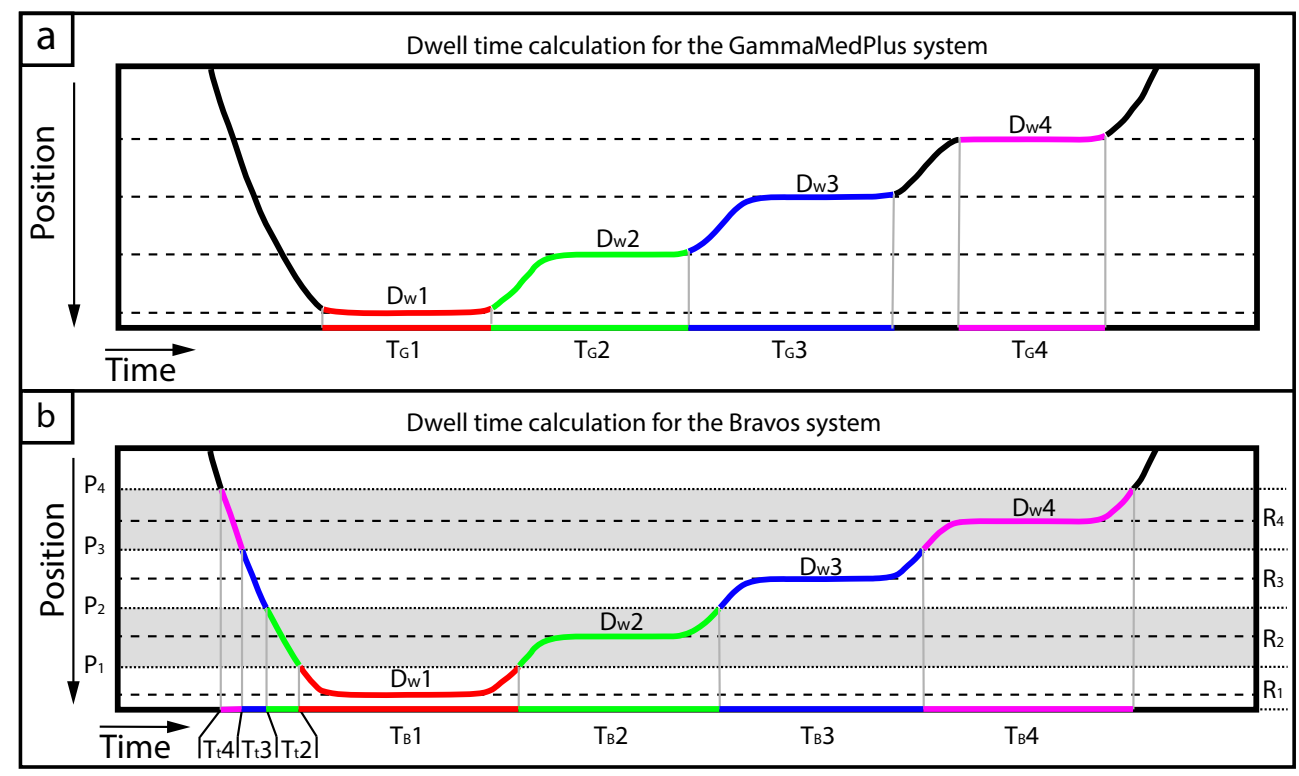

Figure 4.13. Differences in dwell time calculations between a) GammaMedplus iX and b) Bravos systems.

making the dwell time equal to $\mathrm{T}_{\mathrm{B}} 3+\mathrm{T}_{\mathrm{t}} 3$.

For the last dwell position $\left(\mathrm{D}_{\mathrm{w}} 4\right)$, one could expect that the position $\mathrm{P}_{4}$ would be defined at $1.5 \mathrm{~cm}$ from the dwell position Dw4 since the source moves back to the afterloader after $\mathrm{D}_{\mathrm{w}} 4$, however, $\mathrm{P}_{4}$ is actually defined at the same from $\mathrm{D}_{\mathrm{w}} 4$ as $\mathrm{P}_{3}$, making the dwell time equal to $\mathrm{T}_{\mathrm{B}} 4+\mathrm{T}_{\mathrm{t}} 4$.

The reformulated algorithm has a more realistic approach by considering the time that the source spends within a region close to the dwell position as the dwell time.

\subsubsection{Source speed profiles}

The source movement for the Bravos afterloader was measured with a high speed camera (960 fps) for several IDDs and dwell times (the experimental setup is described in section 4.5.2.1). It was measured that the source moves in a uniformly accelerated movement with $|\mathrm{a}|=152 \pm 4 \mathrm{~cm} / \mathrm{s}^{2}$ until reaching the midpoint between dwell positions or maximum speed of $100 \mathrm{~cm} / \mathrm{s}$.

Speed profiles were not measured for the GammaMedplus iX afterloarder. Nevertheless, the user guide [79] describes the same source movement, however with a maximum speed of $63 \mathrm{~cm} / \mathrm{s}$ and $|\mathrm{a}|=154 \mathrm{~cm} / \mathrm{s}^{2}$.

Therefore, the source movement can be mathematically predicted for both afterloaders. 


\subsubsection{Source movement prediction}

The mathematical model for the source movement updated the source position every $1 \mathrm{~ms}$. The movement was split into two parts (separated by the vertical dashed line from Figure 4.14), when the source is moving from the afterloader to the most distal position (most distal dwell position $+1.0 \mathrm{~mm}$ overshoot), and when the source is moving back from the most distal position, stopping at each dwell position. Since the purpose of this study is to evaluate the impact that the transit time algorithm has on the dose distribution, the acceleration adopted for both afterloaders was $154 \mathrm{~cm} / \mathrm{s}^{2}$ because the acceleration specified by the manufacturer for the GammaMedplus iX afterloader (154 $\mathrm{cm} / \mathrm{s}^{2}$ ) is within the experimental uncertainty of the acceleration measured for the Bravos afterloader $\left(152 \pm 4 \mathrm{~cm} / \mathrm{s}^{2}\right)$. Regions evaluated had maximum source speed lower than 63 $\mathrm{cm} / \mathrm{s}$ (maximum GammaMedplus iX source speed), and the most distal dwell position was always at $130 \mathrm{~cm}$, since the GammaMedplus iX afterloader always send the active source to $130 \mathrm{~cm}$ before moving to the most distal dwell position while the Bravos afterloader extends the source until the most distal dwell position but not beyond that. These restrictions were adopted in order to have the same source movement prediction for both afterloaders when there is no transit time correction.

The source movement was initially defined without transit time correction. Then, the transit time was calculated for each dwell position and the source movement was updated removing the transit time from its corresponding dwell time in order to have a dwell time (time in dwell position + transit time) as planned. Figure 4.14 shows an example of the source movement prediction with and without transit time correction (using the Bravos algorithm) for a plan with dwell positions at 130, 129, 128 and $127 \mathrm{~cm}$ and planned dwell time of 1 second.

The source movement prediction model was validated against high speed camera measurements described in subsection 4.5.2.

\subsubsection{Monte Carlo simulations}

The resulting dose distribution from evaluated plans was simulated with the MCNP6.1 MC code, using a GammaMedplus HDR ${ }^{192}$ Ir source model already validated in section 4.3. The dose distribution was scored in water assuming charged-particle equilibrium (CPE) using a cylindrical scoring grid with $0.1 \mathrm{~mm}$ radial resolution and $0.2 \mathrm{~mm}$ axial resolution around the source axis. A total of 1E9 source particles where simulated for each case, resulting in a statistical uncertainty lower than $0.3 \%$ (Type A $\pm 1 \sigma$ ) for the whole scoring grid. 


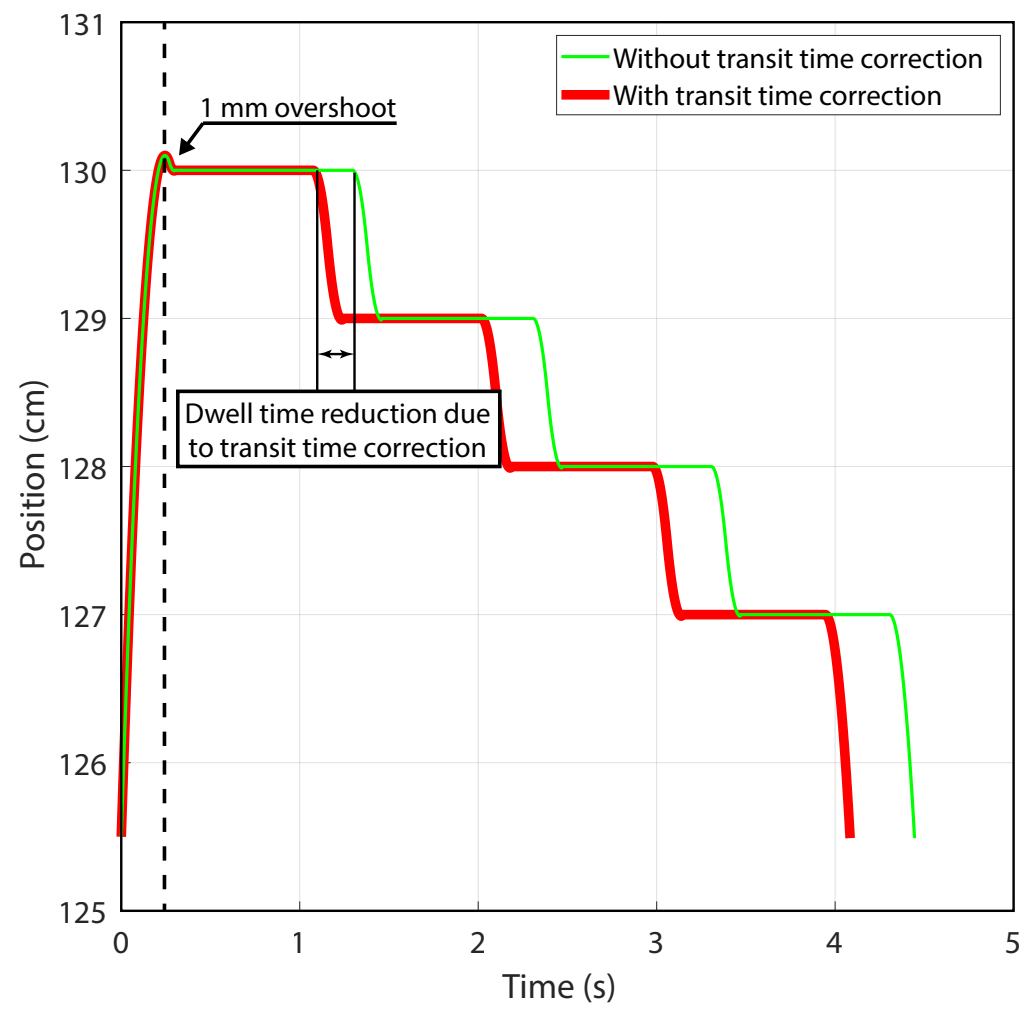

Figure 4.14. Example of source movement prediction with and without transit time correction. The vertical dashed line represents the separation between the model when the source is moving from the afterloader to the most distal dwell position and when the source is moving between dwell positions. The source movement is initially predicted without transit time correction, then the transit time for each dwell position is calculated and removed from the dwell time.

\subsubsection{Evaluated plans}

Plans were created with 10 equally spaced dwell positions following a straight path with the most distal dwell position at $130 \mathrm{~cm}$ and IDDs of 2, 5 or $10 \mathrm{~mm}$. All dwell positions had the same planned dwell time of 1.0, 2.5, 5.0 or 10.0 seconds, and the dose distribution was scored at radial distances of 5,10 and $30 \mathrm{~mm}$ from the source axis, for source movements following the GammaMedplus iX and Bravos transit time correction algorithms. Results were compared to a perfect scenario where there is no transit time and therefore, no transit time correction (which is the situation adopted by TPSs), considering absolute and relative dose deviations for the region between the first and last dwell positions 
5

Results and Discussion 

This section describes the results and discussions for the study subjects presented in this thesis. The discussions are presented after the results of each study.

\subsection{Pretreatment verification system}

This section describes the results and discussions regarding the imaging panel calibration.

\subsubsection{Acquisition rate and region of interest}

Figure 5.1a shows a histogram of the dwell times for the clinical cases (Sk - 40700U) investigated using 0.5 second bins. The maximum dwell time is 46.4 seconds, but very few dwell times exceed 10s (data not shown). Almost $40 \%$ of the dwell times are between 0.1 and 0.5 seconds, which is the most relevant interval for all the cases except the gynecological treatments. Figure $5.1 \mathrm{~b}$ shows a histogram of the distance between the source and the IP for the evaluated cases. The minimal distance is $6 \mathrm{~cm}$ for a head and neck case whose CT images do not include the whole patient geometry. Therefore, we defined the minimal distance of interest as $7 \mathrm{~cm}$ which will cover all the evaluated cases including a reasonable margin. Most of the distances are around $10 \pm 2 \mathrm{~cm}$ for all the treatment modalities and the maximum distance of interest was defined as $22 \mathrm{~cm}$ (16 cm maximum observed distance $+6 \mathrm{~cm}$ margin). The $6 \mathrm{~cm}$ margin is an estimation that should account for thickness of the frame and cushioning we expect to add between the panel and the patient.
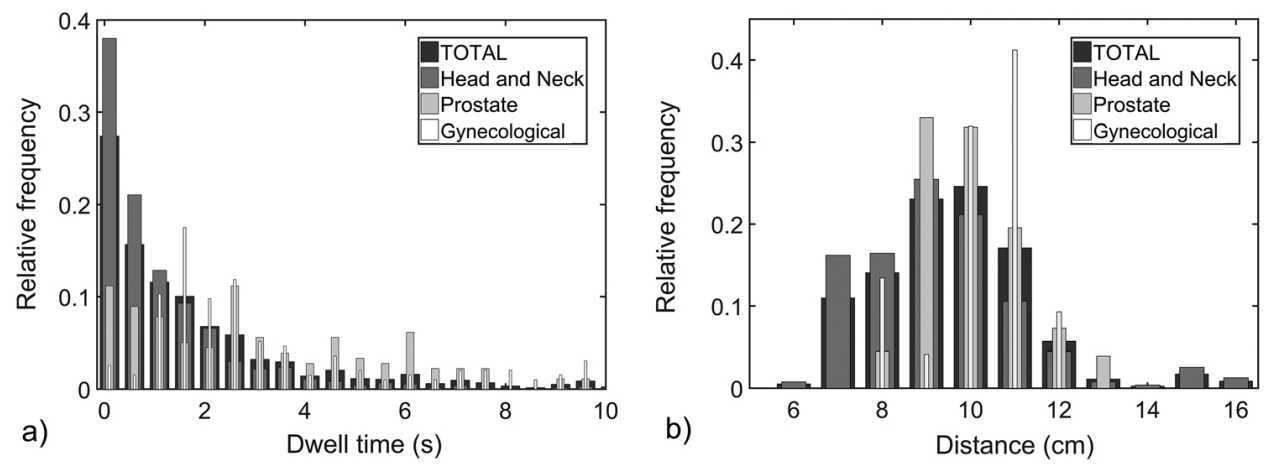

Figure 5.1. Histogram of the dwell time (a) and the distance between the position and the IP (b) for 19 treatment plans including prostate, gynecological and head and neck cases.

\subsubsection{IP response}

Figure 5.2 shows the IP response corrected for the dark field and without applying any additional response corrections. The boundaries of the phantom and scattering from the IP frame of the panel and/or the materials around the water phantom are clearly visible. Response variations can be seen along the whole image including the high dose regions 
at the center. Those variations are related to the different sensitivities of the subpanels composing the detector [123].

A region with maximum dimensions of $16 \times 16 \mathrm{~cm}^{2}$ around the source was defined empirically for fitting purposes. It is sufficiently large to provide a stable fit and receives a relatively high dose not significantly affected by possible variations in the scattering conditions around the phantom. Fits performed using the whole panel had a tendency to show the dwell positions shifted towards one of the boundaries where the scattering was more relevant.

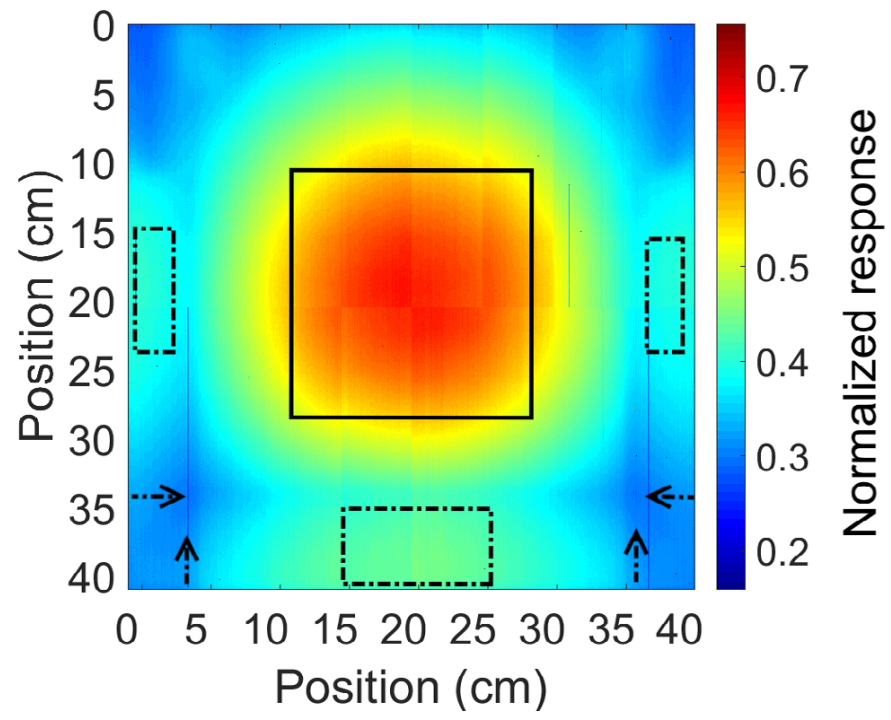

Figure 5.2. IP averaged response over 60 time frames for one source dwell position at $22 \mathrm{~cm}$ from the surface of the panel. The dashed rectangles indicate regions where scattering from the frame of the vertically mounted panel or from the table + holders are clearly visible. The arrows show the boundaries of the phantom. The square at the center of the image shows the region $\left(16 \times 16 \mathrm{~cm}^{2}\right)$ around the dwell position considered for fitting purposes. The panel response was corrected only by the dark field signal.

\subsubsection{Pixel sensitivity map}

The pixel sensitivity map obtained using the irradiations in water (Figure 5.3a) reduced the IP response variation when applied to all the measurements performed either in air or water for different source activities. Figure 5.3b shows a response profile without (Raw) and with the pixel sensitivity correction (Corrected) obtained in water. Note that the correction reduces the noise (see Figure 5.3b inset), but has a very small effect on the shape of the profile. 


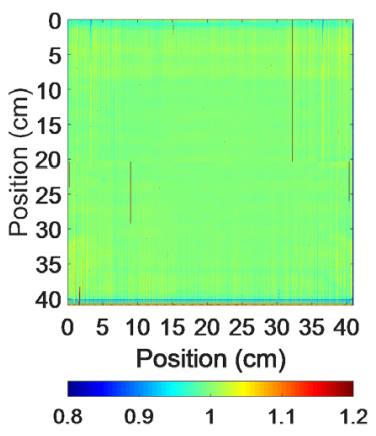

a)

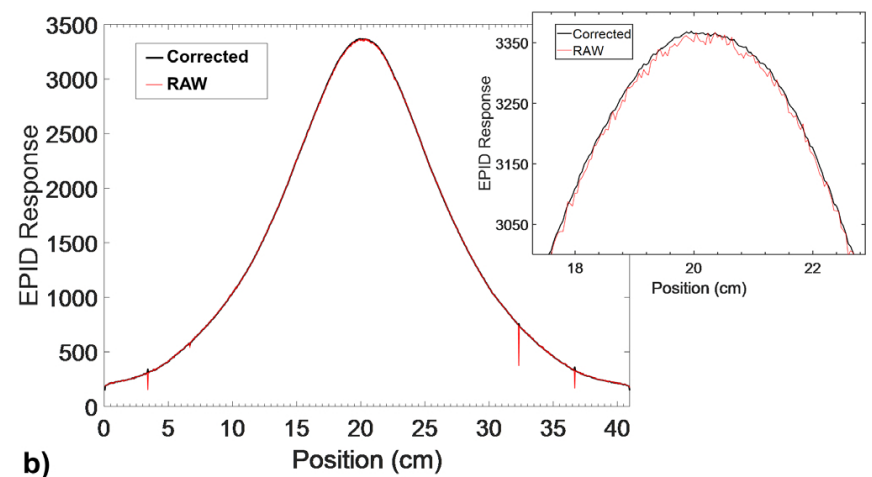

b)

\subsubsection{Response linearity}

The average response of the panel as a function of the distance between the dwell position and the panel is very linear with Sk as shown in Table 5.1. The difference increases with the distance, from less than $0.6 \%$ up to $20 \mathrm{~cm}$ and reaching $1.3 \%$ when the source was at $22 \mathrm{~cm}$ from the panel. Beam softening inside the water phantom didn't affect the linearity of the panel so any distance can be used to measure the source Sk once the panel is calibrated.

Table 5.1. IP response for Sk(Low) (multiplied by 1.67) and Sk(High) for different distances within a water phantom.

\begin{tabular}{cccc}
\hline $\begin{array}{c}\text { Distance } \\
\text { (cm) }\end{array}$ & $\begin{array}{c}\text { Response } \\
\text { Sk(High) }\end{array}$ & $\begin{array}{c}\text { Response } \\
\text { Sk(Low)*1.67 }\end{array}$ & $\begin{array}{c}\text { Absolute } \\
\text { difference (\%) }\end{array}$ \\
\hline 7 & 5040.0 & 5031.1 & 0.18 \\
13 & 1634.8 & 1644.2 & 0.57 \\
20 & 581.0 & 577.8 & 0.56 \\
22 & 441.4 & 435.5 & 1.3 \\
\hline
\end{tabular}

\subsubsection{Source coordinates}

The precision of the robot is better than $0.02 \mathrm{~cm}$ in all directions and is equally matched by its accuracy when using ultrasonic sensors to correct the position. Larger differences were observed due to the sag effect that depends on the payload and the distance from the center of mass, however, this effect can be easily corrected using the positioning sensors to assure the robot accuracy.

The standard deviations of the $\mathrm{x}$ and $\mathrm{y}$ coordinates for the measurements performed in 
water (100 time frames acquired without repositioning the source) are less than $0.02 \mathrm{~cm}$ even for the source-panel distance of $22 \mathrm{~cm}$ and Sk(Low). Therefore, a dwell position can be accurately verified with just one time frame. The standard deviation ( $\mathrm{x}$ and $\mathrm{y}$ coordinates) for 4 consecutive experiments (moving and repositioning the source after each reading $)$ is of the same order $(<0.04 \mathrm{~cm}$ or 1 pixel) indicating that the measurements are reproducible. Figure 5.4a shows the $\mathrm{z}$ coordinate, or the distance between the source and the panel, through the standard deviation $\left(\sigma_{y}\right)$ of the Gaussian fit described in Equation 4.1. Figure 5.4b shows the IP response increasing due to the ghosting effect, whilst the z coordinate, obtained using $\sigma_{y}$ (Eq 3), does not show any clear relation with the acquisition time. Two distances (18 and $22 \mathrm{~cm}$ ) are shown to illustrate the reproducibility of the fit for different distances.
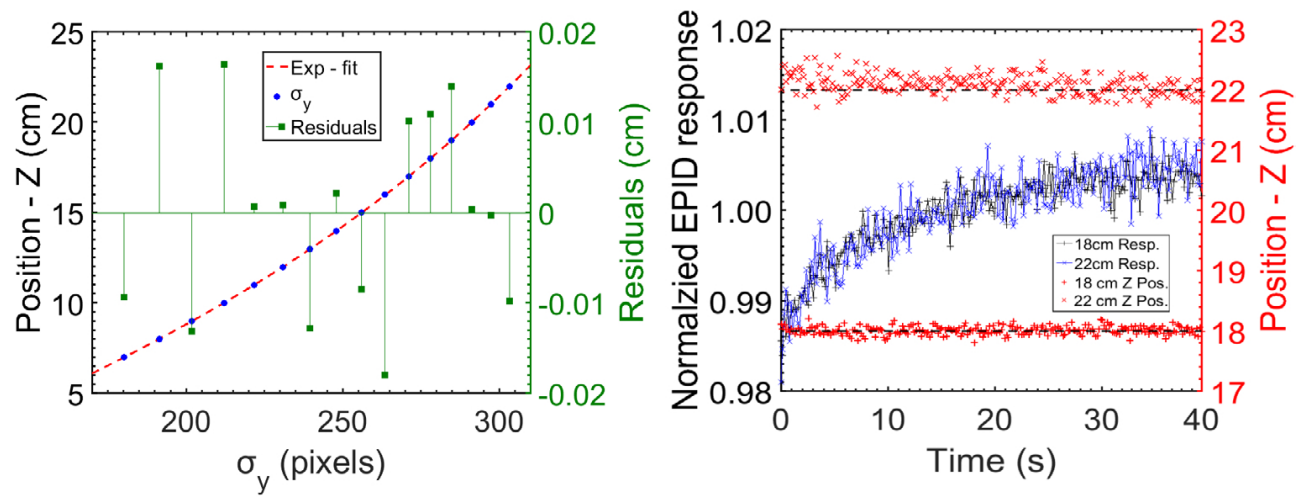

Figure 5.4. a) relation between the standard deviation $\left(\sigma_{y}\right)$ of the Gaussian fit described in Equation 4.1 and the distance between the source and the panel (Equation 5.1). The axis at the right shows the residuals between the experimental values and the fit described in Equation 4.1. b) IP response (connected with a line for visualization purposes, Sk(low), showing an increase with time due to the ghosting effect and the $\mathrm{z}$ coordinate calculated using the standard deviation $\left(\sigma_{y}\right)$ as described in Equation 5.1 The horizontal dashed line (black) represent the expected z position (18 $\mathrm{cm}$ and $22 \mathrm{~cm}$ ).

The exponential fit (Equation 5.1) of the measured sigma vs the $\mathrm{z}$ position was obtained empirically and shows a good agreement with residuals lower than $0.05 \mathrm{~cm}$ (Figure 5.4a). The boundaries of the coefficients are within $10 \%$ for the first exponential and $25 \%$ for the second exponential (95\% confidence interval). The steeper inclination for distances larger than $15 \mathrm{~cm}$ is probably due to variation in the relation between the primary and scattered photon fluence reaching the panel. Results are dependent on direction ( $\sigma_{x}$ or $\sigma_{y}$ ), and the size of the region included in the fit (Figure 5.2). The fit obtained in the direction where the lines of the panel are read out $\left(\sigma_{y}\right)$ was adopted since there is no visible variation due 
to the ghosting effect whilst $\sigma_{x}$ varies (data not show) with the panel ghosting effect.

$$
z\left(\sigma_{y}\right)=2.697 * e^{0.007025 * \sigma_{y}}-12.99 * e^{-0.00897 * \sigma_{y}}\left(R^{2}=1.00\right)
$$

The reproducibility of the $\mathrm{z}$ coordinate measurements varies with the distance and Sk as shown in Table 5.2. The maximum absolute error is the maximum difference for one frame in relation to the reference distance defined using the ultrasonic sensors. The $\mathrm{z}$ coordinate variation increases with the distance and is more relevant for Sk(low), although the difference is less than $0.2 \mathrm{~cm}$ (averaging the frames) for all the evaluated cases. The mean values show good agreement with a maximum difference of $0.06 \mathrm{~cm}$ from the reference except for one measurement (highlighted in the table), which also showed the largest absolute error for one frame. If each frame is evaluated individually (without averaging 100 frames per position), the max absolute error is below $0.2 \mathrm{~cm}$ for all Sk(high) measurements and Sk(low) measurements with distance between the source and the panel less than $19 \mathrm{~cm}$. The maximum observed error for one frame is almost $0.6 \mathrm{~cm}$ due to a high level of noise, which can be reduced by averaging several frames.

Table 5.2. $\mathrm{z}$ coordinates calculated using Equation 5.1 compared to the reference distance of the source measured using an ultrasonic sensor. The mean value corresponds to an average of 100 time frames \pm one standard deviation ( $1 \sigma$ Type A). The maximum absolute error was obtained evaluating each frame individually corresponding to the largest difference between the $\mathrm{z}$ coordinate and the reference distance.

\begin{tabular}{ccccc}
\hline $\begin{array}{c}\text { Reference } \\
\text { Distance }(\mathbf{c m})\end{array}$ & $\begin{array}{c}\text { Sk }=19943 \mathrm{U} \\
\text { Max absolute } \\
\text { error }(\mathbf{c m})\end{array}$ & $\begin{array}{c}\text { Sk }=35816 \text { U } \\
\text { Max absolute } \\
\text { error }(\mathbf{c m})\end{array}$ \\
\hline 7 & $6.98 \pm 0.01$ & 0.03 & $7.00 \pm 0.02$ & 0.10 \\
8 & $8.04 \pm 0.01$ & 0.07 & $8.00 \pm 0.03$ & 0.21 \\
10 & $10.06 \pm 0.01$ & 0.08 & $10.03 \pm 0.03$ & 0.24 \\
12 & $12.05 \pm 0.01$ & 0.08 & $12.02 \pm 0.03$ & 0.21 \\
14 & $14.04 \pm 0.02$ & 0.09 & $14.06 \pm 0.04$ & 0.29 \\
16 & $15.99 \pm 0.03$ & 0.10 & $15.94 \pm 0.05$ & 0.17 \\
18 & $17.95 \pm 0.04$ & 0.12 & $18.01 \pm 0.07$ & 0.21 \\
20 & $19.96 \pm 0.05$ & 0.17 & $19.96 \pm 0.10$ & 0.31 \\
22 & $21.94 \pm 0.07$ & 0.20 & $\mathbf{2 2 . 1 6} \pm \mathbf{0 . 1 6}$ & 0.57 \\
\hline
\end{tabular}

\subsubsection{Dwell positions}

Figure 5.5 shows the source movement along the IP y axis for a programmed irradiation with 5 dwell positions of 9.8 seconds each (for brevity the dwell time measurements are described only for the clinical application see next section) and $0.2 \mathrm{~cm}$ inter-dwell distance. The blue line represents the movement of the source that passes through the dwell positions reaching the end of the needle (around $31.5 \mathrm{~cm}$ ) and then returns to stop 
at the specified positions. The level of the noise between different frames is significantly lower than the difference between the dwell positions so it is clearly possible to distinguish the dwell positions and measure the distance between them. A similar spatial resolution was obtained measuring $\mathrm{x}$ and $\mathrm{z}$ coordinates so the software developed could clearly identify the dwell positions even with the source moving in the 3 coordinates at the same time. The starting point corresponds to the peaks indicated by the triangle pointing downwards. The end of the last dwell position is defined as the final negative peak of the Laplacian (triangle facing upwards).

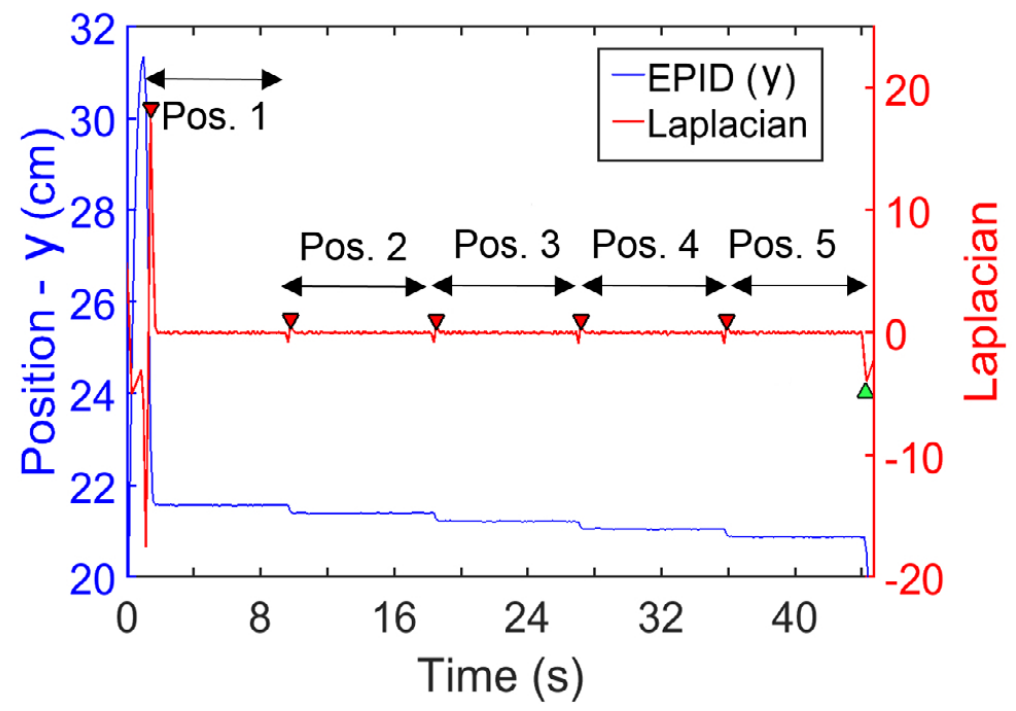

Figure 5.5. Source movement in a needle aligned with the IP y axis (equivalent results were obtained using nonaligned needles) for 5 dwell positions with 9.8 seconds programmed dwell time for each position. The distance between the source and the surface of the panel is $21.6 \mathrm{~cm}$. A continuous line connecting the points was added for visualization purposes.

\subsubsection{Clinical application}

\subsubsection{Absolute coordinates}

The comparison between the measured dwell positions and the expected (treatment plan) positions are shown in Figure 5.6a. The horizontal lines represent $\pm 0.1 \mathrm{~cm}$. The difference for most of the dwell positions are below $0.1 \mathrm{~cm}$ except for a few points. The $\mathrm{z}$ coordinate differences for 3 points (dwell positions 44-46) are about $0.11 \mathrm{~cm}$. In addition, the first dwell position of each catheter shows a larger error for the y coordinate with a negative value indicating the source did not reach the expected dwell position stopping up to 0.2 $\pm 0.1 \mathrm{~cm}$ before. The uncertainty was estimated as $0.1 \mathrm{~cm}$ and is mainly due to the $\mathrm{z}$ 
coordinate component and positioning uncertainties when placing the applicator inside the water phantom.
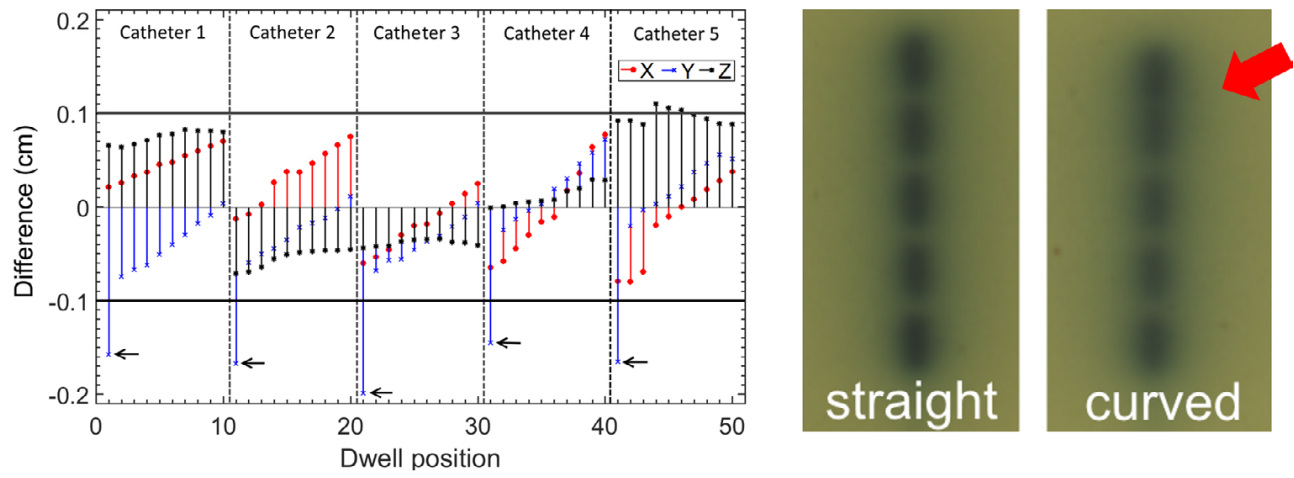

Figure 5.6. a) Error between the expected values (treatment plan) and the measured dwell positions. Results are shown for 5 catheters (vertical dashed lines indicate different catheters) with 10 dwell positions each (arrows indicate the first dwell position of each catheter). b) Film measurements for 5 dwell positions with planned inter dwell distances of $0.5 \mathrm{~cm}$. Results are shown for the transfer tube straight and at the same height as the needle and adding a curvature to the transfer tube to mimic the experiment using the gynecological applicator. The overlap between the first and second dwell position is indicated with an arrow.

The observed difference for the first dwell position of each catheter is due to the curvature of the transfer tube as verified for different inter dwell distances (using the IP) and 0.5 $\mathrm{cm}$ inter dwell distance (radiochromic films + IP). Figure 5.6b shows the result obtained with the films for the transfer tube straight and curved. The overlap between the first and the second dwell position is clearly visible in the film irradiated with the curved transfer tube. The first inter dwell distance is approximately $0.1 \mathrm{~cm}$ shorter than planned. The difference due to the transfer tube curvature is more significant for the first dwell position. The agreement between the inter dwell distances measured using the IP and the films is better than $0.02 \mathrm{~cm}$.

\subsubsection{Simulated error and dwell time verification}

The dwell time measurements with a video camera shows that most of the dwell times are slightly shorter $(<0.05$ seconds $)$ than programmed most likely due to transit time corrections which is small for $0.5 \mathrm{~cm}$ inter dwell distances) applied by the manufacturer $[30,122]$. The programmed dwell times were assumed as reference since the differences measured are much smaller than our experimental uncertainty.

The results do not distinguish the transit time (source movement in between the dwell positions) from dwell times due to the time resolution of the panel. Therefore, the frames acquired with the source in motion, between the dwell positions, have been added to the 
closest dwell position. The average measured dwell time for 197 dwell positions (excluding dwell positions modified for error detection purposes) is $10.0 \pm 0.1$ seconds matching the programmed dwell time (10.0 seconds). All the measured dwell times are within 9.8 and 10.2 (dashed horizontal lines) except for the 9.0 seconds dwell time measured for the third position of catheter 2 and the 11.1 seconds measured for seventh position of catheter 4 (which are the correct results for these positions due to the simulated errors). The total irradiation time shows the same agreement when compared to the programmed values.

Figure 5.7 shows all the measured dwell times for the reference case and case A. Case A's tenth dwell position is missing (arrow 1) indicating that only nine dwell positions were identified, which is correct. The thirteenth dwell time is 9.0 seconds (arrow 2) and the thirty-seventh dwell time is 11.1 seconds (arrow 3). Therefore, all the simulated treatment errors are clearly visible. Note that by verifying only the dwell time it is not possible to identify which dwell position is missing in catheter one. We can only state that catheter one has 9 dwell positions instead of 10 . Therefore, verifying dwell positions $(\mathrm{x}, \mathrm{y}, \mathrm{z})$ is also needed.

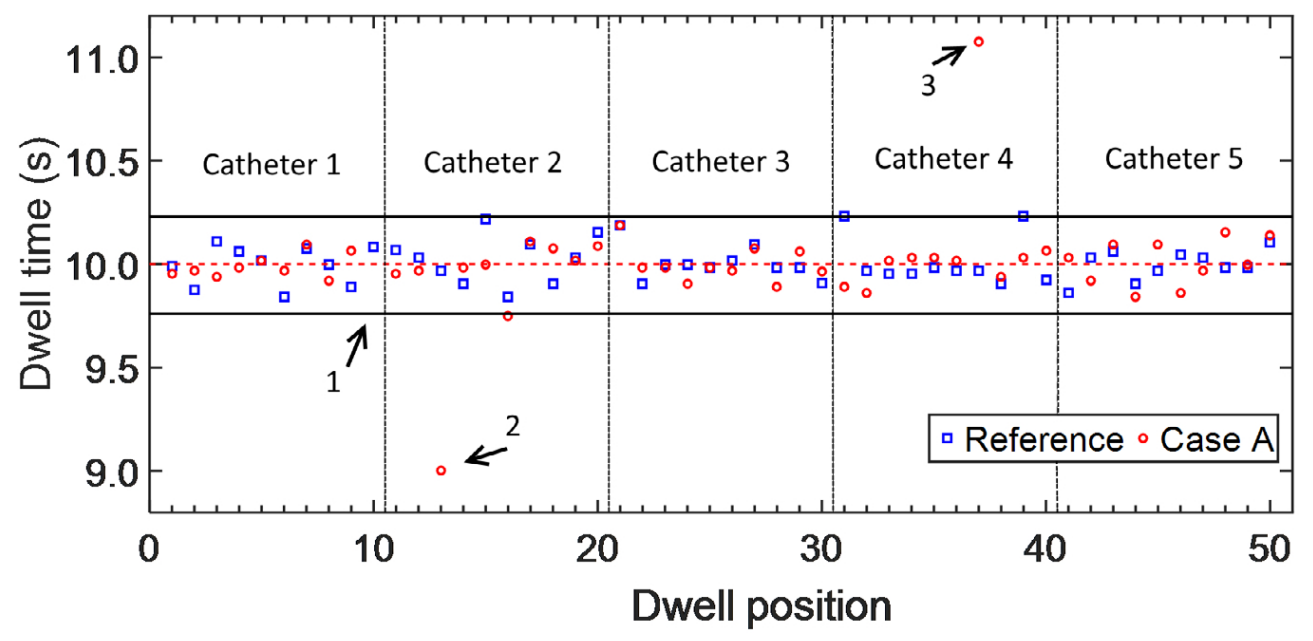

Figure 5.7. Dwell times measured for the reference case and Case A. The horizontal dashed line represents the reference dwell time $(10.0 \mathrm{~s})$ and the continuous horizontal lines define the measurement uncertainties $( \pm 0.2 \mathrm{~s})$. Arrow 1 shows that one dwell position is missing (Case A 1st catheter) whilst arrows 2 and 3 highlight the measurements of the dwell positions for which the dwell times were intentionally modified simulating a treatment error. The vertical dashed lines indicate different catheters.

All the inter dwell distances, except those between the first and second dwell positions of each catheter, measured for the reference case (without any simulated treatment errors) are within $0.19 \mathrm{~cm}$ and $0.21 \mathrm{~cm}$ showing a maximum variation of $0.01 \mathrm{~cm}$ from the expected values. This difference is lower than the source positioning uncertainty 
described by the manufacturer $(1 \mathrm{~mm})$.

Figure 5.8 shows the absolute difference between the reference case and Case A for each measured dwell position. The large y differences (dwell positions 2-10) indicates that one dwell position is missing and a constant difference $(\sim 0.16 \mathrm{~cm})$ indicates that these positions are shifted in Case A (as expected due to the simulated error). The results are very reproducible with a maximum difference of $0.03 \mathrm{~cm}$ for catheters $2-5$ (no simulated positioning errors).

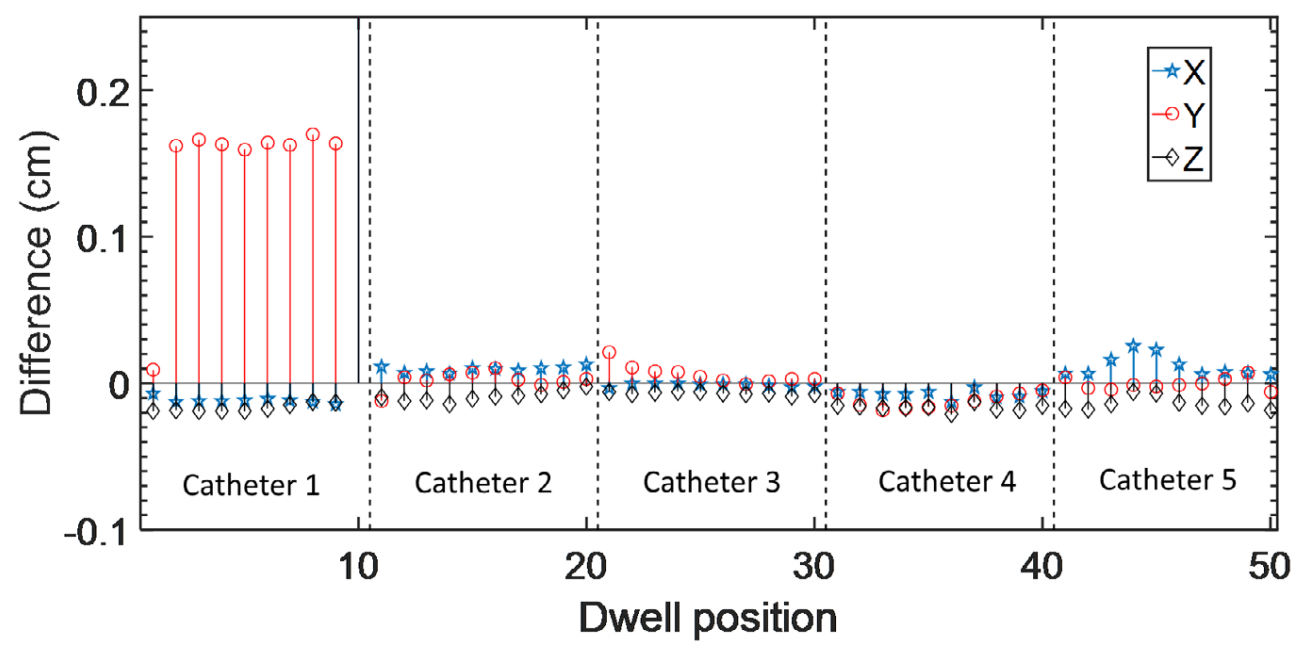

Figure 5.8. Difference between the dwell positions measured for the reference case and Case A. Differences are shown separately for each axis. The vertical dashed lines indicate different catheters

The measured shifts due to the offset added to some catheters (case B) were 0.88, 0.29 and $0.06 \mathrm{~cm}$ (expected values: $1.00,0.40$ and $0.20 \mathrm{~cm}$ ). All the measured shifts are about $0.1 \mathrm{~cm}$ shorter than the expected values, which is consistent with the systematic shift observed for the first dwell position due to the curvature of the transfer tube. We verified that the first dwell position (1st catheter) of case B matches with the sixth position of the reference case, therefore 5 dwell positions were skipped as expected. The same agreement was observed for catheters 4 and 5 with 2 and 1 dwell position skipped respectively .

Case C (transfer tube 2 and 3 were swapped) was the easiest to verify with very large differences against the expected dwell positions. However, it is necessary to use the absolute time of the measurements.

\subsubsection{Discussion}

The use of a robotic arm minimized positioning uncertainties that are very relevant for brachytherapy. The arm was used both for the calibration of the panel, and the positioning 
of the catheters during treatment verification. It is possible to automate the experimental setup reducing the time necessary to calibrate the panel and to perform the measurements for real clinical cases. The processing time per frame is shorter than the acquisition time (for the described IP), therefore it is possible to verify the results in real time and, if necessary, identify the reason of differences between expected and measured values.

The calibration process is essential to assure the necessary accuracy especially for the $z$ coordinate, which is derived from a Gaussian fit, which consist in an empirical fit that may not work if the calibration range is extrapolated outside the intended range. In addition, the calibration is phantom specific since the dimension of the water phantom can affect the results.

The Gaussian fit is sensitive to the nonlinear response of the panel for low signal intensities. The nonlinear effect of one region of the panel was not expected and may be due to the changes in the electronics after years of use. However, the results are very reproducible in water and air and the correction applied reduces drastically the variations. The method employed assures the irradiation was performed with the same energy spectrum changing only the source intensity so the nonlinearity could be measured and corrected. Results published by Smith et al. [49] show a linear response with the source Sk for a different panel. However, it is important to verify if the whole panel shows the same behavior as described in this paper.

Smith et al. described a detailed calibration of an imaging panel, using an ${ }^{192}$ Ir source and additional imaging x-ray source, and its potential application in HDR brachytherapy. The uncertainties described by Smith et al.to measure the $\mathrm{z}$ position are 0.01 and 0.05 and $0.20 \mathrm{~cm}$ (95\% confidence intervalaverage of 30 frames) for $\mathrm{z}$ coordinates of 5,10 and 15 $\mathrm{cm}$, respectively. The standard deviation of the $\mathrm{z}$ coordinates described is this paper are of the same order $(0.02,0.02,0.06 \mathrm{~cm}$ for $\mathrm{z}$ coordinates of 7,10 and $16 \mathrm{~cm}$, respectively $2 \sigma-\mathrm{Sk}$ (high)) with smaller uncertainties than reported by Smith et al.for large distances. This behavior can be explained by the number of frames acquired and by the pixel size of $0.0784 \mathrm{~cm}$ (Smith et al) and $0.04 \mathrm{~cm}$ (this work). Although the calibration methods show some similarities, the goals differ with this study aiming for a feasible pretreatment validation system that can verify treatments plans exported from the treatment planning system. The calibration proposed in this work uses only an ${ }^{192}$ Ir source, since an externalray source may not be available in all the institutions and include only the parameters relevant for pretreatment verification. We introduced a robotic arm in our experimental setup to reduce measurement time, automating part of the process, and increase accuracy. Software was developed to process the frames and to compare information about the treatment to the plan in real time so the clinical staff can verify the results during the 
measurements, reducing the time necessary to process the data.

The measured dwell times show small variations (standard deviation of 0.1 seconds) and a maximum error of 0.2 seconds which are consistent with the acquisition rate of the panel. The observed differences can be attributed to the transit time between the dwell positions since the acquisition rate was not high enough to distinguish dwell and transit time. Therefore, the transit time was added to the closest dwell position depending on the position of the source during the acquisition adding some fluctuation on the dwell time measurement. In principle, with a panel with a higher frame rate, the transit time could be distinguished from the dwell time.

The first calibration of an IP can be time consuming and should include measurements along the ${ }^{192}$ Ir source lifespan ( $~ 3$ months). However, periodic checks (e.g. after replacing a source) can be automated using a robotic arm . In addition, reference values would be available after the first calibration so a few measurements points can be defined instead of performing the whole calibration again.

The proposed methodology allows a pretreatment verification using information from the treatment plan that can be converted to IP coordinates with a CT scan of the water phantom used in the experiment. The uncertainties defining the catheter, measuring the distances between the applicator and the phantom (registered CT images) and reproducing the CT geometry during the experiment can be significant so the process should be carefully verified. The use of a robot arm was essential in the process to assure accuracy and reproducibility so the simulated positioning errors $(>0.1 \mathrm{~cm})$, very close to the declared manufacturer positioning uncertainty $(0.1 \mathrm{~cm})$, are clearly visible. This can be very beneficial to the clinic detecting errors and increasing patient safety.

The large discrepancies observed for the first dwell position were detected comparing the absolute positions (Figure 5.6) and also the inter dwell distances. Therefore, the reason of such discrepancies may be identified using different parameters acquired during the same measurement.

The curvature of the transfer tube affected mainly the first dwell position of each catheter with no clear effect in the remaining dwell positions. The reason of this behavior is not clear, and our hypothesis is that the afterloader pushes the source to the tip of the catheter and the cable follows the external wall of the transfer tube increasing the length of the trajectory, so the source does not reach the expected position. The afterloader then pulls the cable back to the next dwell position and by pulling the cable it can get closer to the inner wall reducing the length of the trajectory.

The CT images of the phantom and applicator where acquired separately so in a real 
clinical case the images acquired for the treatment plan (patient + applicator) can be registered with a CT of the water phantom. This method was used to minimize the impact in the daily routine since medical images of the patient are already available and a reference $\mathrm{CT}$ of the water phantom needs to be acquired only once. Commercial treatment planning systems have several features to register images, which can easily be done by trained users. Treatment planning system registration tools can be used to align the applicator and place it always in the same position related to the phantom walls so the robot can be used to place the applicator in the same position during the experiments.

\subsection{Iridium Imaging system (IrIS)}

This section shows the deviation between measured and planned dwell positions and dwell times, measured with the IrIS system for a tandem applicator model AL07522002 (Varian Medical System) for a plan with 19 dwell positions with IDD of $5 \mathrm{~mm}$ and dwell time of 1 second. Figure 5.9 shows the results for each dwell position (measured 3 times) with an error bar corresponding to 3 standard deviations. In total, 57 dwell positions were evaluated and the maximum absolute deviation from planned dwell position was $1.1 \mathrm{~mm}$ for a single point. All other points are within manufacturer uncertainty specifications of \pm $1.0 \mathrm{~mm}$.

Most dwell times presented an absolute deviation between 0 and $50 \mathrm{~ms}$ from planned, which is close to the IP time resolution ( $\sim 30 \mathrm{~ms}$ at $33 \mathrm{fps}$ acquisition rate) and within the afterloader time resolution of $50 \mathrm{~ms}$.

\subsubsection{Discussion}

The IrIS system has several new features that can increase the precision and accuracy for applicator commissioning. The first one and possibly the most relevant feature is that the IrIS system provides time-resolved information on the source position, which not only allows to verify small interdwell distances but also dwell times. The imaging panel used in this study has a maximum acquisition rate of $33 \mathrm{fps}(\sim 30 \mathrm{~ms}$ resolution) and pixel size of $0.14 \mathrm{~mm}$, which is enough to detect deviations in cases adopting minimum dwell times ( 0.1 seconds) and IDDs ( $1 \mathrm{~mm}$ ) allowed by the TPS. Furthermore, the 2D gaussian fit provides subpixel accuracy on dwell position determination. Nevertheless, the acquisition frame rate should be adjusted according to the planned dwell time to reduce the amount of storage space required to save the data.

Another advantage of the IrIS system is that, after the initial setup where the background and applicator projection are acquired (which would take around $5 \mathrm{~min}$ ), the operator doesn't need to go inside the room after each irradiation (unlike QA using radiochromic films). The plan can be repeated several times while the system processes the data in 


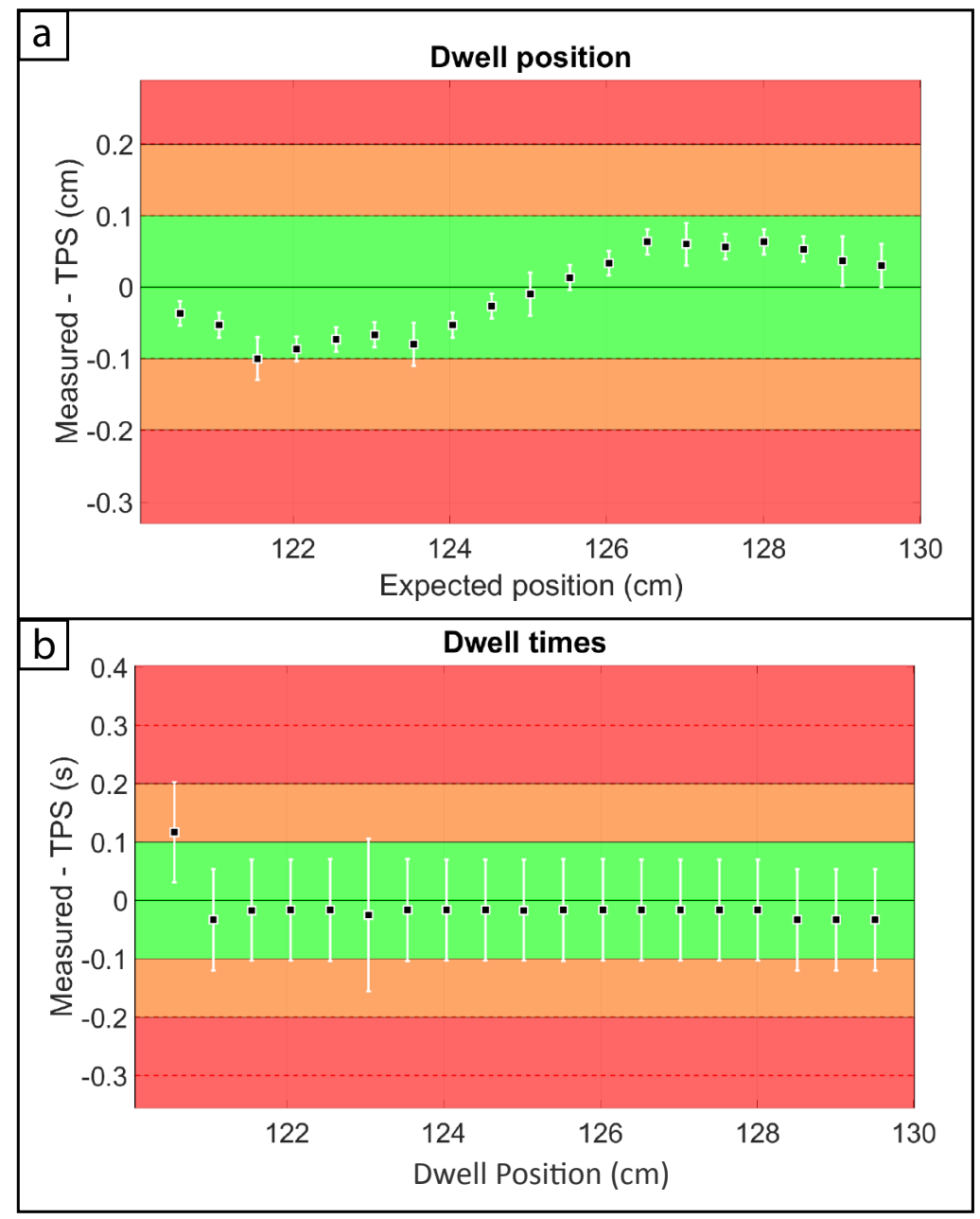

Figure 5.9. Deviation between measured and planned (a) dwell positions (b) and dwell times for a tandem applicator model AL07522002 (Varian Medical Systems). The deviation between measured and planned values was measured 3 times for each dwell position and the error bars correspond to 3 standard deviations.

real time. Therefore, after initial setup, the time for each measurement is essentially the time required to run the QA plan, allowing to perform several measurements and have better statistics for the QA. Fonseca et al. [17] reported that an intermittent problem was detected for one of the applicators evaluated, suggesting that 2-3 measurements may not be enough for a proper QA, and encouraging to perform several measurements to detect problems that don't occur very often.

Furthermore, the IrIS system automatically registers the plan from the TPS on top of the 
applicator projection using the radiopaque markers as reference. Such feature is not only convenient from a workflow point of view, but it also excludes the possibility of human errors when measuring the difference between measured and planned dwell positions.

The disadvantages of the IrIS system are that each applicator requires its own holder, and from an economic point of view, the initial (and relatively high) cost of an imaging panel. However, the use of an IP reduces (or even excludes) the use of (disposable) radiochromic films for QA, and also reduces the labor hours required for applicator QA. Hence, it is expected that the IrIS system would pay for itself after a period that will differ between institutions depending on how often QA is performed.

\subsection{Designing a rectal applicator for contact brachytherapy, a proof of concept}

\subsubsection{Design optimization}

MC simulations in water showed that the dose falloff in the applicator central axis is not only $\mathrm{d}_{\text {tip-tip }}$ dependent, but also depends on the number of channels used. For designs having from 1 to 20 channels, $\mathrm{d}_{\text {tip-tip }}$ between $1.5 \mathrm{~mm}$ and $3.5 \mathrm{~mm}$ resulted in dose falloff fairly similar to the one of the P50 device.

As the number of channels in the applicator increases, it becomes possible to add more concentric rings to accommodate the channels and move the sources closer to the applicator exit surface without violating the dose uniformity criterion RSD.

The channels were distributed as follows:

1 channel - a central channel with $d_{\text {tip-tip }}=3.5 \mathrm{~mm}$;

2-5 channels - a ring (without central channel) with $d_{\text {tip-tip }}=3.5 \mathrm{~mm}$;

6-13 channels - a central channel and 1 ring with $\mathrm{d}_{\text {tip-tip }}=2.5 \mathrm{~mm}$;

14-20 channels - central channel and 2 rings with $d_{\text {tip-tip }}=1.5 \mathrm{~mm}$.

The transition when there were enough channels to add a new ring results in steps in RSD (from to the change in number of rings) (Figure 5.10a) and irradiation time (from

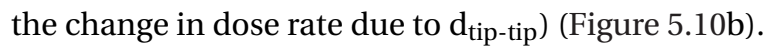

The horizontal dashed lines in Figure 5.10a show the maximum value allowed for RSD (2\%), where models with 8 channels or more were acceptable. The same criterion (lower limit of 8 channels) is represented by the vertical dashed line in Figure 5.10b, showing the treatment delivery time for a Sk of $20350 \mathrm{U}$ (5Ci) source lower limit of 8 channels. As the number of channels increases the irradiation time has the tendency to be lower due to the higher dose rate as the sources are brought closer to the surface, however the total treatment time has the tendency to keep rising due to the dummy check time component. 
The RSD for applicator designs with 8, 9, 10 and 11 channels were $1.93 \%, 1.88 \%, 1.88 \%$ and $1.88 \%$, respectively. The 9 channels model (one ring of 8 channels and one central channel) was found to be the best compromise between RSD and treatment time.
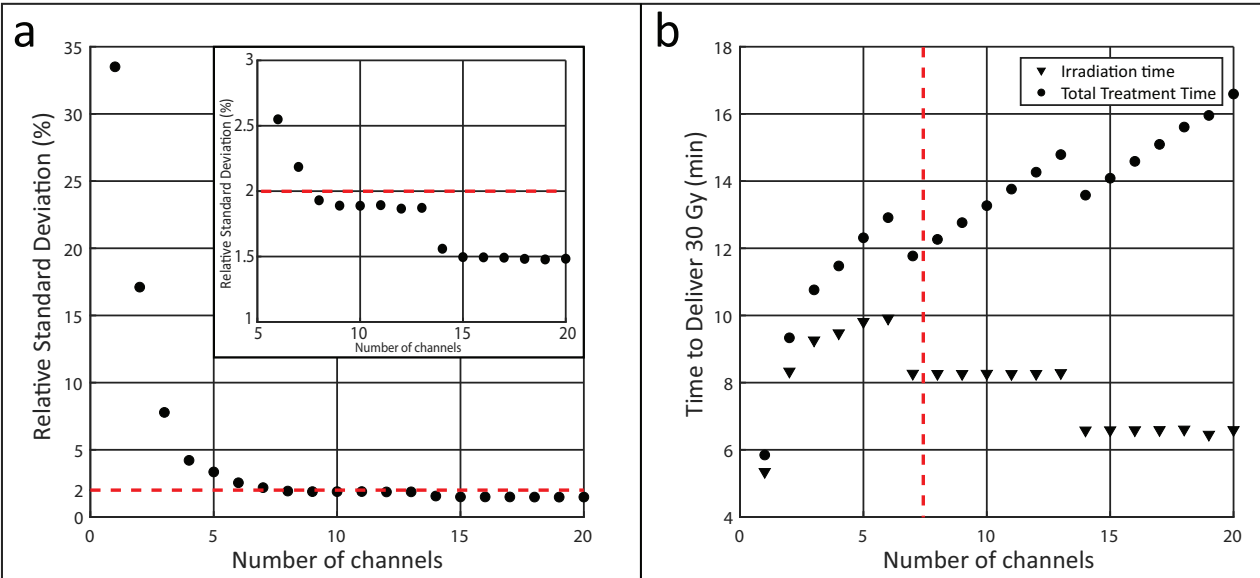

Figure 5.10. RSD at the applicator's exit surface with a rescaled insert to better evaluate applicators with more than 5 channels (A) and treatment time to deliver $30 \mathrm{~Gy}$ with a Sk of $20350 \mathrm{U}$ (5 Ci) source (B) obtained from optimizing applicators with a number of channels ranging from 1 to 20 . The reference line in A (horizontal dashed line) for RSD of $2 \%$ represents the arbitrary dose uniformity criterion. The vertical dashed line in B represents the applicators accepted by the same criterion.

Figure 5.11a shows the applicator's final design, with 9 channels, $3 \mathrm{~mm}$ of pure tungsten $\left(19.25 \mathrm{~g} / \mathrm{cm}^{3}\right)$ for lateral shielding $(1.5 \mathrm{~mm}$ around the applicator core and $1.5 \mathrm{~mm}$ belonging to the proctoscope) and a $1 \mathrm{~mm}$ thick plastic cap to absorb secondary electrons $[124,125]$ (composition by weight $37.5 \% \mathrm{C}, 3.2 \% \mathrm{H}, 59.3 \% \mathrm{~F}$, with a density of $1.8 \mathrm{~g} / \mathrm{cm}^{3}$, as used in the Leipzig and Valencia applicators). Figure $5.11 \mathrm{~b}$ shows the applicator positioning inside the rectum against the tumor. The knee-chest position is adopted for treatments with the P50 machine; however, lateral and lithotomy positions may be also an option for the new applicator. Possible treatment positions will be subject of further studies.

\subsubsection{Experimental validation}

It is important to verify the achievable positioning accuracy of the source near the tip of the applicator since this will influence the dose accuracy. The mean value measured of $\mathrm{d}_{\text {tip-tip }}$ with a video camera for multiple irradiations with the prototype applicator was $(3.00 \pm 0.15) \mathrm{mm}$ for an intended $\mathrm{d}_{\text {tip-tip }}$ of $3.00 \mathrm{~mm}$. Table 5.3 shows the gamma analysis [135, 136] with $1 \mathrm{~mm} / 3 \%$ and $1 \mathrm{~mm} / 1 \%$ acceptance criteria between the experimental and simulated dose distributions (simulation as reference) for a MC model of the simple experimental setup using $\mathrm{d}_{\text {tip-tip }}=3.0 \mathrm{~mm}$. 


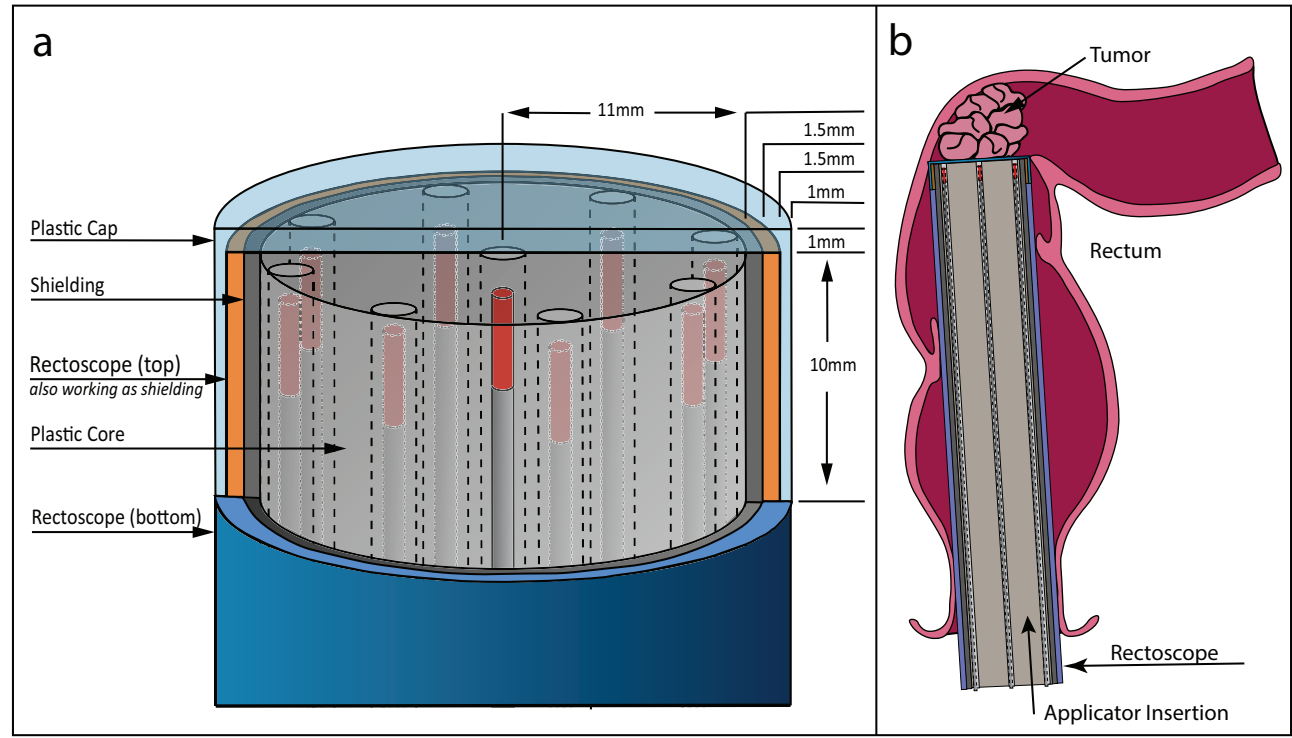

Figure 5.11. A Model for the 9-channel HDR contact applicator. A central channel surrounded by a ring of 8 equally distributed channels at $10 \mathrm{~mm}$ radii. B Applicator positioning inside the rectum against the tumor.

Table 5.3. Gamma analysis (percentage of dose values passing the acceptance criteria) between simulated and experimental data for 9 channel irradiations for scoring planes at 1,2,5 and $10 \mathrm{~mm}$.

\begin{tabular}{ccccc}
\hline & & & \multicolumn{2}{c}{$\gamma$ Acceptance Criteria } \\
\hline Irradiation & Dwell time (s) & Scoring Plane $(\mathrm{mm})$ & $1 \mathrm{~mm} / 3 \%$ & $1 \mathrm{~mm} / 1 \%$ \\
\hline 1 & 25 & 10 & 100.0 & 98.6 \\
2 & 25 & 10 & 100.0 & 99.8 \\
3 & 25 & 10 & 100.0 & 99.2 \\
\hline 4 & 10 & 5 & 100.0 & 92.4 \\
5 & 10 & 5 & 99.9 & 88.6 \\
6 & 10 & 5 & 99.9 & 84.2 \\
\hline 7 & 20 & 2 & 99.8 & 96.2 \\
8 & 20 & 2 & 99.9 & 96.1 \\
9 & 10 & 2 & 99.9 & 83.3 \\
\hline 10 & 20 & 1 & 98.9 & 93.3 \\
11 & 20 & 1 & 99.1 & 91.8 \\
12 & 20 & 1 & 99.3 & 93.2 \\
\hline
\end{tabular}

Figure 5.12 shows a comparison between simulated and experimental dose distributions for irradiation 12 from Table 5.3. Figure 5.12a shows an experimental dose distribution that is asymmetric at the central region due to source inclination and possible $\mathrm{d}_{\text {tip-tip }}$ variations among the channels, nevertheless, presenting good agreement between simulated $\left(\mathrm{d}_{\text {tip-tip }}=3.0 \mathrm{~mm}\right)$ and experimental results for isodoses lower than $14 \mathrm{~Gy}$. Figure $5.12 \mathrm{~b}$ shows the experimental dose profile encompassed by the simulated dose 
profiles along the horizontal line in Figure 5.12a. Multiple dose profiles $\left(\mathrm{d}_{\text {tip-tip }}=2.85,3.00\right.$ and $3.15 \mathrm{~mm}$ ) were simulated to represent the uncertainty of the $\mathrm{d}_{\text {tip-tip }}$ measuring $( \pm 0.15$ $\mathrm{mm}$, type B) as error bars on the simulated profile. The MC uncertainty was lower than $0.1 \%$ (type $\mathrm{A} \pm \sigma$ ) in the scoring region, negligible compared to the uncertainty due to $\mathrm{d}_{\text {tip-tip variation. }}$

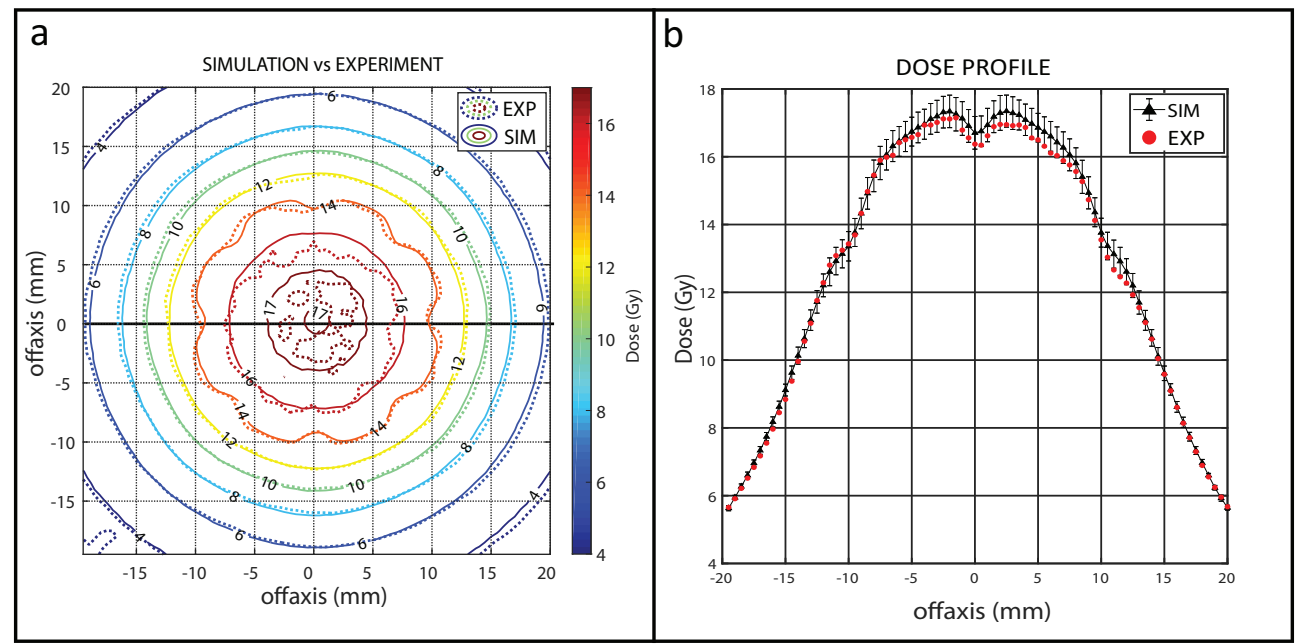

Figure 5.12. Comparison between simulated and experimental dose distributions for the 12th irradiation of Table 5.3, at $1 \mathrm{~mm}$ depth. a) isodoses comparing experimental (dotted lines) and simulated (continuous lines) dose distributions; b) simulated dose profile along the line at (a) for $\mathrm{d}_{\text {tip-tip }}=(3.00 \pm 0.15) \mathrm{mm}$, compared to the experimental dose profile.

\subsubsection{Source inclination effect}

The values of $\Delta \mathrm{D}_{\% \max }$ and $\phi_{\max }$ (from Figure 4.8) were evaluated for a total of 17 single channel irradiations and the mean value of $\Delta \mathrm{D}_{\% \max }\left(\Delta \mathrm{D}_{\% \mathrm{avg}}\right)$ was $(4.8 \pm 0.7) \%$, showing little or no dependence on setup variations. The value of $\phi_{\max }$ was highly sensitive to setup variations and is essentially random.

The $\Delta \mathrm{D}_{\% \max }$ value simulated for source inclinations $\left(\theta_{\mathrm{s}}\right)$ from 0 to $5 \mathrm{deg}$ at scoring planes at $1,2,5$ and $10 \mathrm{~mm}$ resulted in a linear behavior of $\Delta \mathrm{D}_{\% \max }$ vs $\theta_{\mathrm{s}}$ expressed by $\Delta \mathrm{D}_{\% \max }$ $=(2.16 \pm 0.01)^{*} \theta_{\mathrm{s}}$, which implies an experimental source inclination for single channel irradiations of $(2.2 \pm 0.3) \mathrm{deg}$.

The source inclination effect can be detected and simulated; however, it cannot be predicted since the rotation $(\phi)$ of the source is random. The source inclination $\left(\theta_{\mathrm{s}}\right)$ is in part due to the imperfect soldering between the source cable and the source, and also due to cable snaking effects. Figure 5.13 shows the comparison between MC dose calculations of the HDR applicator without shielding at $1 \mathrm{~mm}$ scoring plane, with sources 
inclinations $\left(\theta_{\mathrm{s}}\right)$ of 0 and $2.2 \mathrm{deg}$. Cold and hotspots over the sources are apparent in Figure 5.13, with differences of up to $2 \%$ in dose locally, however, all the dose values passed a gamma analysis with $1 \mathrm{~mm} / 1 \%$ acceptance criteria between both dose distributions. Reducing the source inclination is desired to reduce local dose deviations, however it doesn't significantly influence the overall dose distribution.

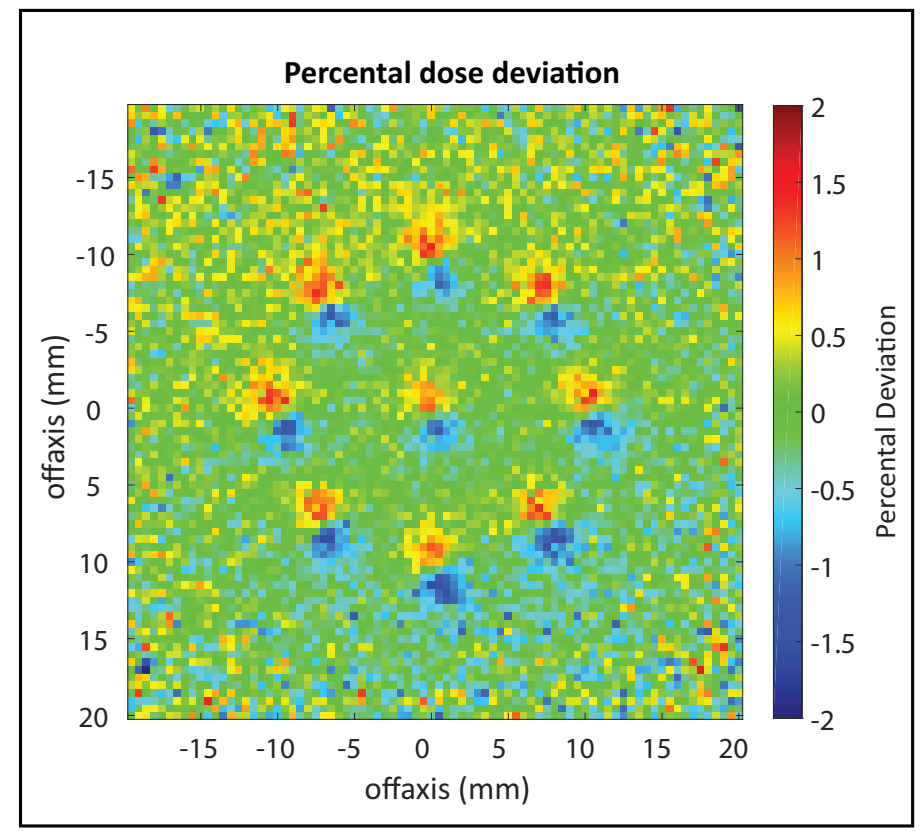

Figure 5.13. Simulation of the dose deviation that a source inclination of 2.2 deg would cause in the dose distribution at $1 \mathrm{~mm}$ depth when compared to a source perfectly perpendicular to the treatment surface $\left(\theta_{\mathrm{S}}=0 \mathrm{deg}\right)$.

\subsubsection{Virtual shielded model}

Figure 5.14a shows the simulated dose at the virtual shielded applicator's exit surface (Figure 5.11), where hotspots are noticeable close to the source channels. Figure 5.14b shows the depth isodoses in front of the applicator. The dose profiles in Figure $5.14 \mathrm{c}$ at the applicator's surface and at $1 \mathrm{~mm}$ depth along line 1 from Figure 5.14a (connecting the center of the central source and the center of two other sources) show that the effect of these hotspots are greatly reduced at $1 \mathrm{~mm}$ depth and are also reduced for the dose profile along line 2 from Figure 5.14a passing in between two sources (rotated 22.5 deg; Figure 5.14d).

Figure 5.15 shows a comparison of dose profiles at different depths and dose falloff simulated in water between the P50 contact therapy device with the $22 \mathrm{~mm}$ proctoscope 


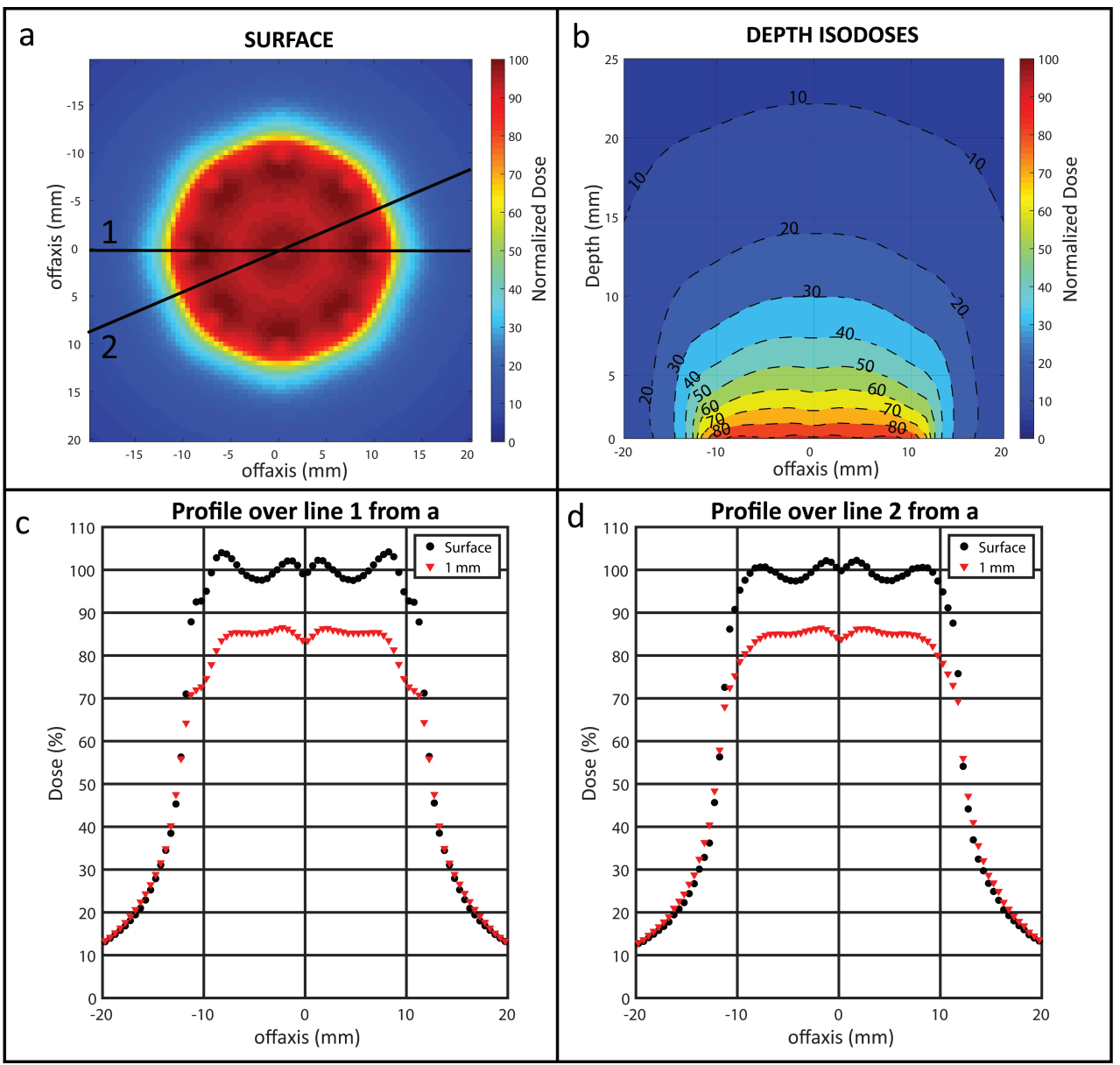

Figure 5.14. Simulated normalized dose at the applicator surface for a region of $40 \times 40 \mathrm{~mm}^{2}$ (a), depth isodoses distribution (b), dose profiles at the applicator surface and at $1 \mathrm{~mm}$ depth in water along the lines 1 (c) and 2 (d) from (a).

(data from Groce et. al. [137]) and the HDR applicator. The dose profiles from Figure 5.15 $(a, b, c)$ show that the radiation beam resulting from the HDR applicator has a wider aperture than the one generated by the P50 machine, nevertheless with a similar dose falloff (maximum difference of 4.5\% at $12 \mathrm{~mm}$ depth) shown in Figure 5.15d.

\subsubsection{Discussion}

\subsubsection{Design and clinical implementation}

The applicator can be integrated to currently available treatment planning systems with model-based dose calculation algorithms (MBDCAs), such Acuros BV in BrachyVision (Varian) and Oncentra Brachy ACE (Elekta, Stockholm, SE). Studies have shown small 


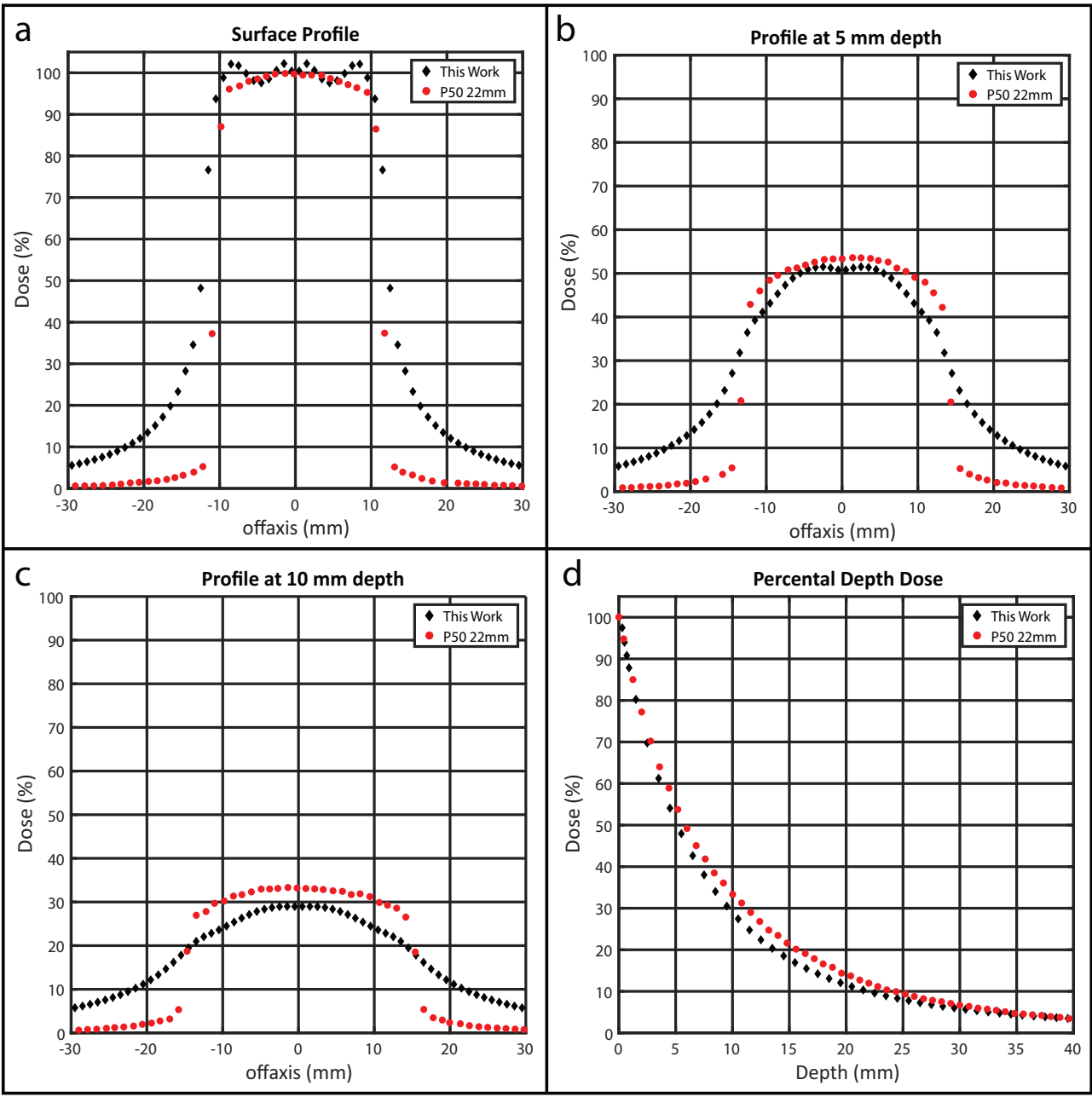

Figure 5.15. Comparison between dose distributions generated by the Papillon 50 device with a 22 mm proctoscope (Croce et. al. [137]) and the HDR applicator.

deviations in dose distribution between Monte Carlo simulations and these treatment planning systems [46, 47] for shielded applicators. The use of multiple channels and the possibility of TPS integration enables a better control of dose distribution, which is an advantage comparing to current $\mathrm{CXB}$ techniques. However, the time required to send the dummy sources was the main factor that limited modeling an applicator with more than 9 channels (Figure 5.10b), having more channels in the applicator would increase the deg of freedom available for the TPS. Another limiting factor to use more channels was the transit dose contribution to the total dose (contribution equivalent to extra dwell time of $0.84 \pm$ 0.09 seconds for the first dwell position using a Sk of $40700 \mathrm{U}$ source)[134]. Increasing 
the number of channels would reduce the dwell time per channel and the transit dose contribution would be higher, especially for the most distal dwell positions that have no transit time correction in current afterloaders. Moreover, the literature mentions a range of transit times for afterloaders of the same model, as summarized by Fonseca et al.[122]. Ideally, an afterloader with transit dose correction for the most distal position and reduced dummy time would enable a design with more channels.

\subsubsection{Validation}

For a total of 12 irradiations using 9 channels (Table 5.3), the 3 irradiations where less than $90 \%$ of the voxels passed a gamma analysis with $1 \mathrm{~mm} / 1 \%$ acceptance criteria had dwell times of 10 seconds per channel (irradiations 5, 6 and 9) with a transit dose contribution of $6.5 \%$ to the total dose. For dwell times of 20 seconds or higher (average transit dose contribution of $2.5 \%$ ), an average of $96 \%$ of the voxels passed using the same criteria. This deviation is due to a higher uncertainty on the transit time correction for lower dwell times, and the fact that transit time correction applied for the HDR applicator was measured for a different geometry [134] and may not be correct for the present applicator. The usual dose delivered in clinical practice for rectal cancer patients using CXB is $30 \mathrm{~Gy}$ [132], which requires an average dwell time per channel of 25 seconds to be delivered with the HDR applicator using a Sk of $40700 \mathrm{U}$ source. Therefore, applying the transit dose correction from Jeong et al.[134] is precise enough for typical treatment doses, however further investigation of transit dose specific for the HDR applicator is required for low dose treatments with a high activity source, which is the worst case scenario for the transit dose correction.

\subsubsection{Source position uncertainty}

A $\mathrm{d}_{\text {tip-tip }}$ between $2 \mathrm{~mm}$ and $3 \mathrm{~mm}$ is required to obtain a steep dose falloff, mainly governed by the inverse-square law. However, due to the high dose gradient close to the source, the mean dose difference at the applicator exit surface is approximately $1.7 \%$ for each $0.1 \mathrm{~mm}$ shifted from $\mathrm{d}_{\text {tip-tip }}=2 \mathrm{~mm}$ to $3 \mathrm{~mm}$. The source position can be shifted due to transfer tube bending, and forcing the source against the end of the channel would remove the uncertainty of $d_{\text {tip-tip }}$, however it would also increase the potential of snaking of the source cable inside the channel, causing a local dose distribution inhomogeneity due to source inclination, or it could cause an error on the afterloader due to obstruction detection. Since the designed applicator has straight rigid channels, the option of using smaller lumen sizes is under evaluation to reduce the snaking effect when the source reaches the tip of the channel. This would reduce the errors due to transfer tube bending, that may occur during clinical practice. 


\subsubsection{Virtual shielded model}

The dose distribution for an applicator with 9 channels has the smallest RSD $3 \mathrm{~mm}$ away from the sources tip and the dose falloff will be similar to the one generated by P50 when $d_{\text {tip-tip }}$ is between 2 and $3 \mathrm{~mm}$. Therefore, it is possible to choose $\mathrm{d}_{\text {tip-tip }}$ with conventional treatment planning systems in order to control the tissue depth where the dose distribution will be most uniform and the shape of the dose falloff. The HDR applicator has a wider radiation beam than the P50 machine due to the small $\mathrm{d}_{\text {tip-tip. }}$ If the proctoscope is also used as shielding, it can be placed around a protruding tumor providing better shielding for healthy tissues and shaping a narrower radiation field.

The material considered as shielding for all the MC simulations was pure tungsten $\left(19.25 \mathrm{~g} / \mathrm{cm}^{3}\right)$. In practice, tungsten is a material that is not easily manufactured, and it is possible that a tungsten alloy will be adopted for the real applicator. Nevertheless, commonly used tungsten alloys often have up to $97 \%$ of tungsten in their composition, providing similar shielding effect.

\subsubsection{Clinical aspects of dose delivery}

The designed applicator represents a new design in rectal cancer brachytherapy. The applicator can be positioned directly against the tumor by visual-guidance through a proctoscope. This approach enables fast and reliable positioning on the tumor without the need for additional imaging devices. Furthermore, the proctoscope encompassing the applicator enables shielding in all directions except towards the treatment depth into the rectal wall containing the tumor, which is the main advantage of this applicator compared to other HDR techniques, the possibility to more selectively target the tumor (due to shielding and lower beam aperture). Having a steeper dose falloff allows us to destroy the tumor cells layer by layer, which also preserve healthy tissues including organs like the vagina/urethra, prostate and bladder. This is extremely important as after conventional HDR brachytherapy late toxicity, mainly rectal bleeding, is a real problem [139]. Late rectal bleeding is not so common after treatment with the P50 machine. It has been hypothesized that this is due to a significantly lower dose in the vascular plexus in the outer layer of the intestinal wall .

Due to its new design, clinical data of patients treated with this applicator are not yet available. For the first clinical applications the patient eligibility criteria are expected to be similar to those applied for patients treated with the P50 machine (well to moderately well-differentiated rectal adenocarcinoma, cT1-3 tumors with less than $3 \mathrm{~cm}$ in diameter situated less than $12 \mathrm{~cm}$ from anal verge [139]) once the dose distributions are similar. Already planned future studies will show whether the eligibility criteria should be adapted. 
To date limited information is available about the dose distribution in the tumor of the treatments with the P50 device. There are no guidelines for dose prescription or reporting in rectal endoluminal contact of brachytherapy. Questions include especially how the dose should be distributed among the volumes to increase tumor cure while avoiding significant toxicity. As a starting point, we tried to mimic a dose distribution comparable to the Papillon $50 \mathrm{kV}$ contact therapy, as much clinical experience is available for this approach [153-162] as monotherapy or combined with other forms of radiotherapy. Our approach mimics the $50 \mathrm{kV}$ contact therapy well, although not perfectly, especially with an even somewhat steeper dose falloff in depth and a somewhat less steep dose falloff in the penumbra to the side. Although differences are small, clinical studies are needed to evaluate whether these are relevant or not.

\subsection{Advanced design of a rectal applicator for contact brachytherapy}

\subsubsection{Experimental validation}

\subsubsection{Source Position Verification}

A total of 250 dwell positions were measured during the experiment after the source path was optimized, for which $22(8.8 \%)$ presented an absolute deviation from planned dwell position greater than $1.0 \mathrm{~mm}$, with a maximum deviation of $1.2 \mathrm{~mm}$ for a single dwell position. Out of these 22 deviations greater than $1.0 \mathrm{~mm}, 19$ were from measurements from channels 1 and 5 from Figure 5.16, which have the largest curvature among the channels evaluated.

Figure 5.16 shows the overlap of the applicator, optimized source path, planned and measured dwell positions. The deviations between planned and measured dwell positions for the channels with lowest and greatest deviations are plotted separately.

\subsubsection{Film Dosimetry}

Figure 5.17 shows the isodoses and global dose ratios $^{1}$ for dose distributions measured at $1 \mathrm{~mm}, 2 \mathrm{~mm}$ and $5 \mathrm{~mm}$ depth in solid water, for which the reference dose $\mathrm{e}^{2}$ was 28.9, 24.8 and 16.9 Gy, respectively. The maximum distance between measured and simulated isodoses for depths of $1 \mathrm{~mm}, 2 \mathrm{~mm}$ and $5 \mathrm{~mm}$ had local variations of up to $(0.8 \pm 0.2) \mathrm{mm}$, $(0.9 \pm 0.2) \mathrm{mm}$ and $(0.7 \pm 0.2) \mathrm{mm}$, respectively, for the $90 \%$ isodoses. Such deviations were more likely to appear along the y direction of Figure 5.16 since this is the direction most affected by the uncertainty on the source dwell position of $\pm 1 \mathrm{~mm}$. Moreover, the slanted edge of the applicator and shielding creates a bigger space between the shielding

\footnotetext{
${ }^{1}$ Difference between experimental and simulated values, divided by the reference dose.

${ }^{2}$ Average dose within the $90 \%$ isodose for each depth.
} 


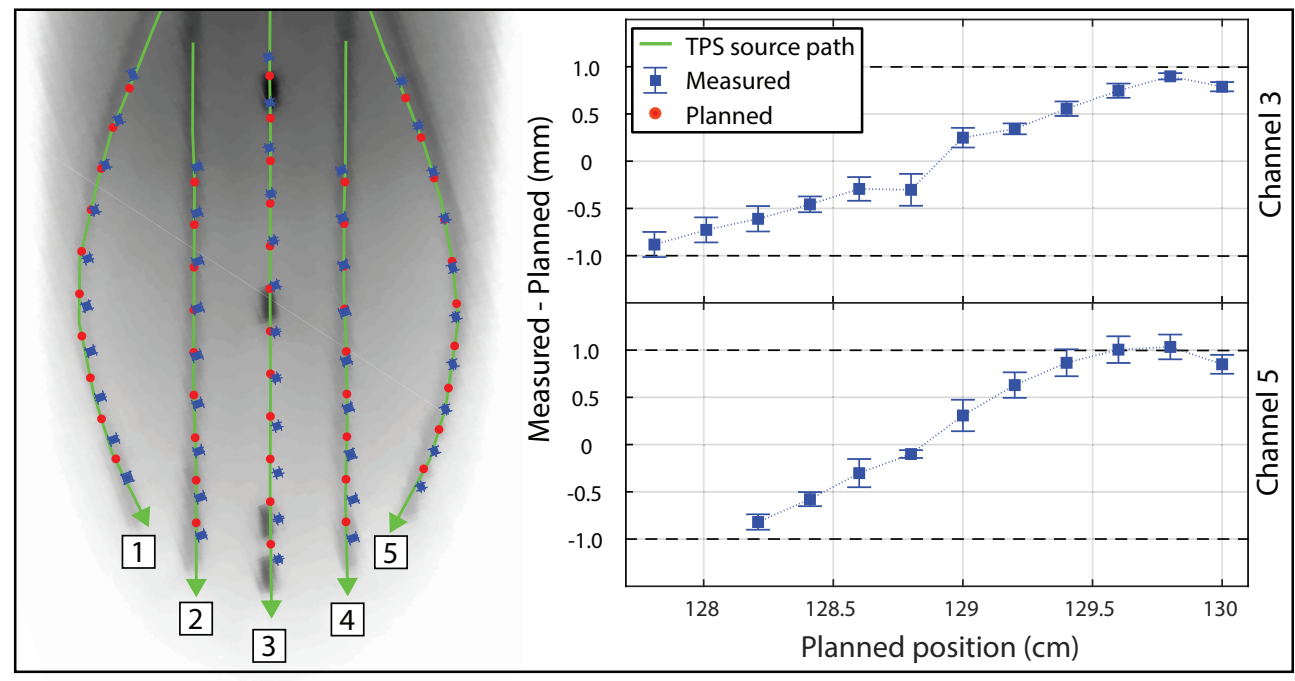

Figure 5.16. Deviation between measured (IrIS) and planned dwell positions after source path optimization, which was performed by changing the source path on the TPS after each measurement until the average deviation between measured and planned dwell positions was minimized. The image on the left shows a projection of the treatment region of the applicator with dummy sources inserted into the channels (with the only purpose of highlighting the channels that were not clearly visible), as well as the optimized channel path, planned and average measured dwell position over 5 measurements (error bars of 2 standard deviations). The plots on the right show the deviation for channels 3 and 5, which presented the lowest and greatest deviations, respectively.

and the applicator core along the y direction, resulting in an uncertainty position of the shielding of $\pm 0.10 \mathrm{~mm}$ in the $\mathrm{x}$ direction and $\pm 0.15 \mathrm{~mm}$ in the $\mathrm{y}$ direction.

The maximum deviation between measured and simulated dose distributions occurs for measurements at $1 \mathrm{~mm}$ depth, with global dose differences reaching $6 \%$ of the reference dose of 28.9 Gy. Nonetheless, 98.6, 99.5 and $100 \%$ of the voxels passed in a gamma analysis (results not shown) with acceptance criteria of DD/DTA $(3 \% / 1 \mathrm{~mm})$ at depths of $1 \mathrm{~mm}, 2 \mathrm{~mm}$ and $5 \mathrm{~mm}$, respectively. Combined estimated uncertainties for the film measurements range from $2.2 \%$ to $7.3 \%$ (Table C.3), for which uncertainties type A were lower than $2 \%$. Moreover, the uncertainty on the source position according to the afterloader manufacturer is $\pm 1 \mathrm{~mm}$ [163]. Hence, the acceptance criteria of DD/DTA (3\%/1 mm) were chosen to be conservative.

The gamma analysis for the dose leakage experiment measured at radial distance of 1 $\mathrm{mm}$ and $2 \mathrm{~mm}$ from the shielding resulted in $96.8 \%$ and $94.7 \%$ of the voxels passing acceptance criteria of DD/DTA (3\%/1 mm), and maximum global dose ratios of $5.4 \%$ and $4.2 \%$, respectively, for a reference dose of $24.8 \mathrm{~Gy}$ (planned dose at $2 \mathrm{~mm}$ depth). 


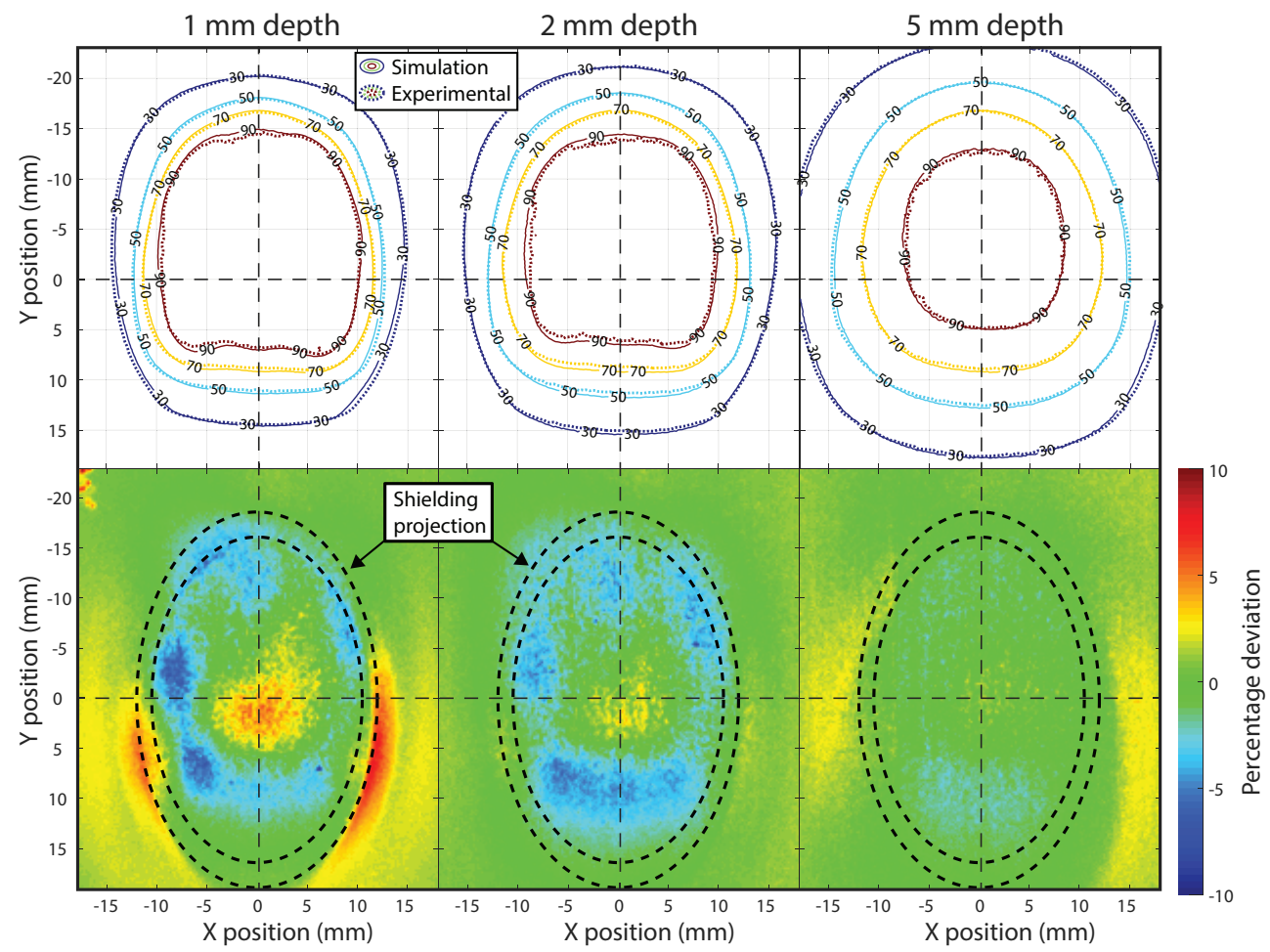

Figure 5.17. Isodoses and global deviation (i.e. 100*(Exp-Sim)/Ref. Dose) between measured and simulated dose distribution at depths of $1 \mathrm{~mm}, 2 \mathrm{~mm}$ and $5 \mathrm{~mm}$, adopting reference doses of 28.9, 24.8 and 16.9 Gy, respectively. Each plot has the geometric center of the contact surface adopted as reference axis (represented by vertical and horizontal dashed lines) with the same applicator orientation shown in Figure 5.16.

\subsubsection{Simulation of the virtual model of the applicator}

Figure 5.18a shows that the center of the simulated dose distribution at $2 \mathrm{~mm}$ depth (intersection between lines L2 and L3) is at a distance of (5.2 \pm 0.2$) \mathrm{mm}$ from the geometric center of the contact surface (intersection between lines L1 and L2). This follows from the design of the applicator (as shown in section 4.4.1) where the slanted edge does not allow dwell positions close to the edge of the contact surface at the most distal end of the applicator, as shown in Figure 4.9c. Nevertheless, such a shift is constant for all depths evaluated, as shown by the dose profiles and isodoses along line L2 (Figure 5.18c and d). The $90 \%$ isodose at $2 \mathrm{~mm}$ depth covers a region of $(21.6 \pm 0.2) \mathrm{mm}$ along line $\mathrm{L} 2$ and 18.2 $\pm 0.2 \mathrm{~mm}$ along line L3.

The resulting dose falloff scored at the center of the dose distribution (Figure 5.18b) is 


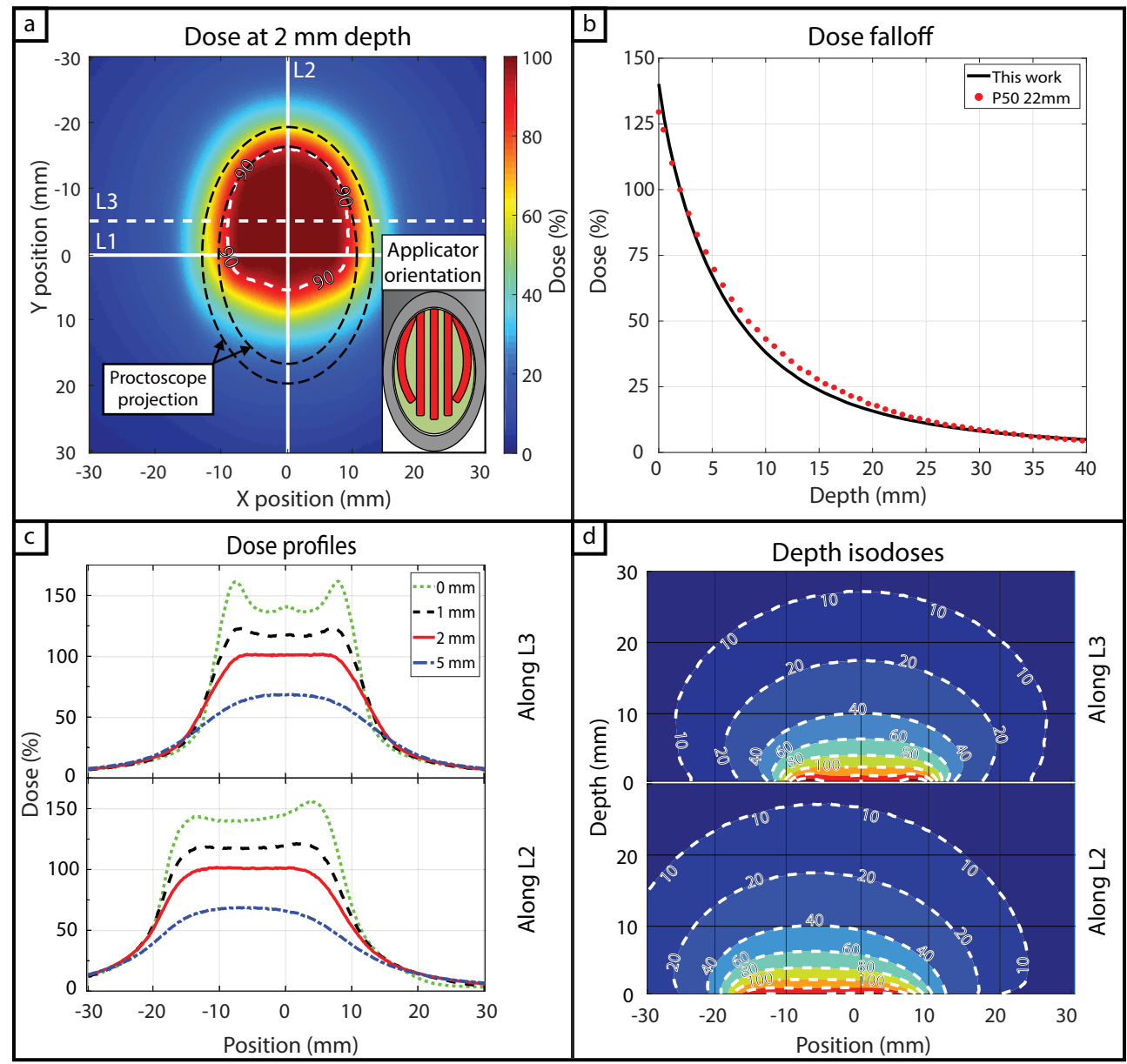

Figure 5.18. Simulated dose distribution for the MAASTRO applicator with the geometric center of the contact surface adopted as reference axis. a) Dose distribution at $2 \mathrm{~mm}$ depth showing the geometric center of the contact surface (intersection between lines L1 and L2), the center of the dose distribution (intersection between lines L2 and L3) and the 90\% isodose; b) Dose falloff along the center of the dose distribution compared to the dose falloff of the Papillon 50 device with a 22 $\mathrm{mm}$ applicator; c) Dose profiles at $0 \mathrm{~mm}, 1 \mathrm{~mm}, 2 \mathrm{~mm}$ and $5 \mathrm{~mm}$ depths along lines L2 and L3 and, d) Depth isodoses along lines L2 and L3.

slightly steeper than the one produced by the Papillon 50 (P50) device with a $22 \mathrm{~mm}$ applicator [137]. Normalized at $2 \mathrm{~mm}$, the dose falloff for depths of 0, 5, and $10 \mathrm{~mm}$ are $130 \%, 70 \%$, and $43 \%$ for the P50 with a $22 \mathrm{~mm}$ applicator and $140 \%, 67 \%$, and $38 \%$ for the MAASTRO applicator, respectively. CXB treatments usually prescribe a surface dose of 30 Gy [74, 139], meaning that 23.1 Gy would be delivered at $2 \mathrm{~mm}$ depth according to the P50 dose falloff used as reference. The MAASTRO applicator can deliver 23.1 Gy at 2 $\mathrm{mm}$ depth in approximately $3 \mathrm{~min}$ of irradiation time using a $40700 \mathrm{U}$ (10 Ci) source or 6 
min with a $20350 \mathrm{U}$ (5 Ci) source. Nevertheless, the time required to perform obstruction verifications (dummy checks) with the GammaMedplus iX afterloader is approximately 30 seconds per channel, which increases the treatment time (irradiation + dummy check) by $2.5 \mathrm{~min}$ for the MAASTRO applicator. The P50 surface dose rate ranges from 20 to 35 Gy/min depending on the applicator adopted [138], resulting in a irradiation time of less than 1 minute and 30 seconds for the applicator with lowest dose rate (30 $\mathrm{mm}$ applicator). Simulated dose profiles at $0 \mathrm{~mm}, 1 \mathrm{~mm}, 2 \mathrm{~mm}$ and $5 \mathrm{~mm}$ depths along lines L2 and L3 (Figure 5.18c), show that the surface dose is not uniform (oscillating along line L3 between $136 \%$ and $160 \%$ of the reference dose at $2 \mathrm{~mm}$ depth), however, such fluctuation is reduced between $123 \%$ and $117 \%$ at $1 \mathrm{~mm}$ depth.

The dose leakage for a $0.2 \mathrm{~mm}$ thick layer around the proctoscope (Figure 5.19) is greater close to the 2 channels adjoining the edges of the treatment surface with maximum leakage hotspots reaching $49 \%$ of the planned dose at $2 \mathrm{~mm}$ (hotspots are indicated by arrows in Figure 5.19) . Nevertheless, the dose leakage quickly drops to less than $30 \%$ (less than $7 \mathrm{~Gy}$ for a typical CXB treatment) for regions that are $5 \mathrm{~mm}$ or further from the contact surface (distance pointed by the dashed arrow in Figure 5.19). These dose values, however, do not include the extra dose from secondary electrons generated in the proctoscope. The leakage dose for regions with $30 \%$ leakage or more are, on average, increased by $9 \%$ (i.e. $30 \%$ leakage will become $32.7 \%$ ) due to secondary electrons for the first $0.2 \mathrm{~mm}$ layer around the proctoscope. The electron contribution to the leakage dose drops, however, from $9 \%$ to less than $1 \%$ (becoming negligible) when dose is scored between $0.6 \mathrm{~mm}$ and $0.8 \mathrm{~mm}$ from the proctoscope.

\subsubsection{Discussion}

The MAASTRO applicator was evaluated with two series of experimental validations to verify the dwell position accuracy and dosimetry. This was followed by MC simulations of the virtual (CAD) model of the applicator to characterize the dose distribution expected for the final manufactured product.

\subsubsection{Experimental validation}

\section{Source Position Verification}

Local deviations between measured and planned dwell positions for curved applicators are well known and reported in the literature [25, 77, 164]. Nevertheless, the average absolute deviation between measured and planned dwell positions after source path optimization for the channels with the highest deviations ( 1 and 5 from Figure 5.16) was (avg. (range) $0.7(0.1-1.2) \mathrm{mm}$ and the average absolute deviation for the remaining 


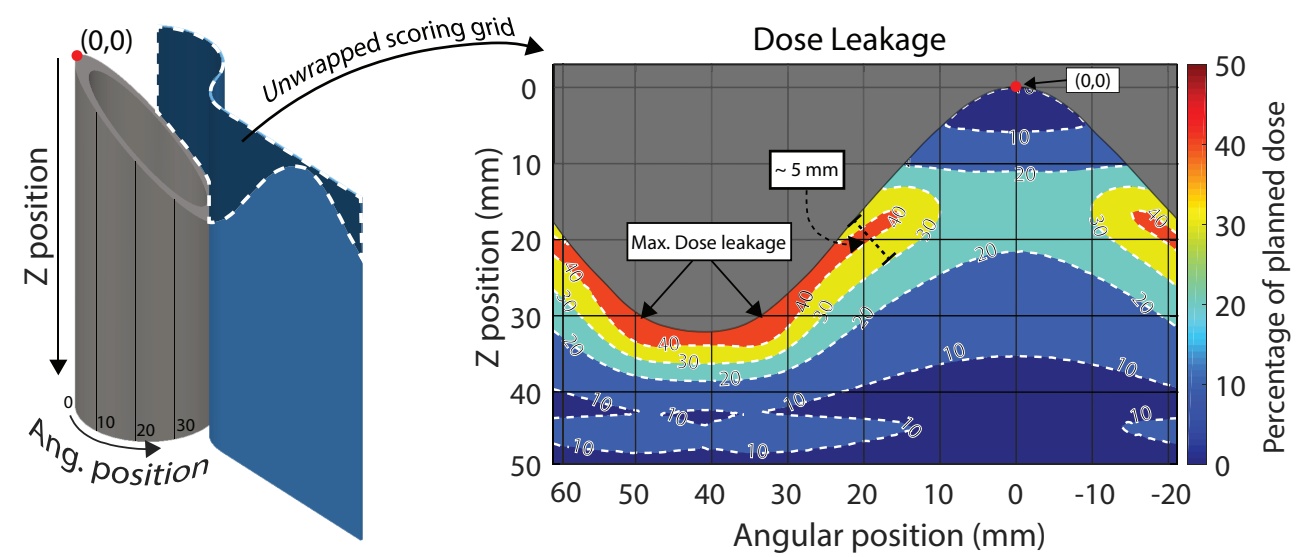

Figure 5.19. Simulated dose leakage around the proctoscope for a $0.2 \mathrm{~mm}$ thick cylindrical scoring grid. The cylinder with a slanted edge shows how the scoring grid was wrapped around the proctoscope, having the sharp edge of the proctoscope adopted as reference position $(0,0)$ for the scoring grid. The region above the proctoscope edge was masked for better evaluation of dose leakage through the proctoscope wall.

channels was 0.5 (0.0-1.1) $\mathrm{mm}$, showing no relevant statistical difference among channels after the applicator was properly commissioned [17]. Moreover, the deviations between measured and planned dwell positions are within the afterloader precision of $\pm 1.0 \mathrm{~mm}$ [163].

\section{Film Dosimetry}

Local discrepancies between MC simulations and experiments with film dosimetry reach a maximum of $6 \%$ at $1 \mathrm{~mm}$ depth close to the shielding (see Figure 5.17). Such local deviation is believed to be due to a small shift in the shielding position or inclination that could have a large impact on the local dose distribution at $1 \mathrm{~mm}$ depth since it is a region of steep dose gradient. Nevertheless, the region affected by the shielding passes the gamma analysis while the points that fail the gamma analysis are in the treatment region, indicating that the uncertainty on the distance between the source and the scoring plane plays a bigger role on the overall dose distribution. Nevertheless, such variations are not clinically relevant since the first few milimeters of the tumor will be removed after each fraction anyway.

\subsubsection{Simulation of the virtual model of the applicator}

Simulations show that the MAASTRO applicator can generate a radiation field suitable for treatment of small rectal tumors, presenting a steep dose falloff similar to those of $50 \mathrm{kVp}$ CXB devices. However, the contact surface of the applicator has a cold region that must be accounted for. For this reason, the proctoscope will have clearly visible engravings to 
guide its placement against the tumor considering the high and low dose regions of the contact surface.

The longer treatment time for the MAASTRO applicator ( 8 minutes and 30 seconds using a $20350 \mathrm{U}$ source) is acceptable for a brachytherapy treatment, however it will require extra care to safeguard that the applicator will not move during the irradiation.

The greater (when compared to P50) and heterogeneous surface dose distribution is unlikely to jeopardize a treatment outcome since experiences with the P50 device show that the first few mm of the tumor are removed after each fraction [74] and, P50 delivers a surface dose greater than 30 Gy for tumors that protrude into the applicator [165]. Moreover, the relatively wide beam aperture (Figure 5.18-c and d) may be beneficial to treat microscopic residual tumors, which extends from the gross tumor by $4.7 \mathrm{~mm}$ on average [166]. Moreover, a study by Reniers et. al. [167], has reported that 50kVp x-rays emitted by an Axxent (Xoft Inc., California, US) electronic BT source may have a biological effect approximately $40 \%$ higher than the photons from a HDR ${ }^{192} \mathrm{Ir}$ source. However, clinical studies are necessary to evaluate such effects.

\subsection{Mechanical evaluation of the Bravos brachytherapy system}

\subsubsection{Experiments using the CamScale device}

For every case evaluated, the reported deviations by the CamScale from each of the target positions $(90 \mathrm{~cm}, 120 \mathrm{~cm}$ and $150 \mathrm{~cm}$ ) had always a similar value (within a range of $\sim 0.2$ $\mathrm{mm}$ from each other). Hence, the results shown for dummy and source position deviations combine the deviations for the 3 target positions in what follows.

\subsubsection{Reproducibility and inter-channel variations}

The system was calibrated using channel 1 before starting the measurements. Figure 5.20 shows the deviation from target position for the source and dummy cables with 3 measurements for every odd channel. Channels with more measurements (not shown in Figure 5.20) follow the same pattern observed for the other channels. The median deviation reported for all source cable measurements combined is $0.0 \mathrm{~mm}$ with a maximum absolute deviation of $0.4 \mathrm{~mm}$ for $4 \%$ of the positions. The median deviation for the dummy cable was $0.2 \mathrm{~mm}$, with $17 \%$ of the deviation exceeding $0.4 \mathrm{~mm}$ and the maximum deviation measured was $0.7 \mathrm{~mm}$ for $2 \%$ of the positions.

Position verifications performed using channel 15 after system calibration using channel 1 resulted in an average deviation of $(0.23 \pm 0.11) \mathrm{mm}$ for the dummy cable and $(0.16 \pm 0.08)$ $\mathrm{mm}$ for the source cable. When channel 15 was used for system calibration and position verification, the average deviations were $(0.00 \pm 0.14) \mathrm{mm}$ for the dummy cable and $(-0.08$ 


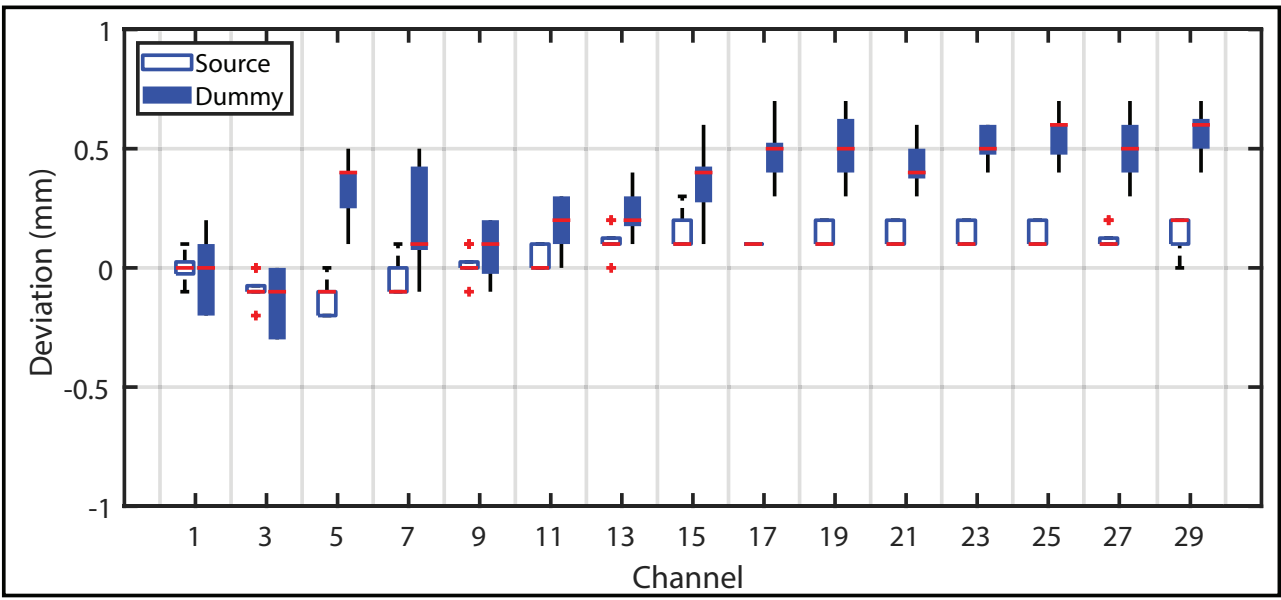

Figure 5.20. Boxplot showing the deviation from target positions $(90,120$ and $150 \mathrm{~cm})$ measured with the CamScale ( 3 times each) for the source and dummy cables for every odd channel.

$\pm 0.05) \mathrm{mm}$ for the source cable. Results show a smaller deviation from target positions when the same channel is used for both calibration and position verification.

Alternating between channels 1 and 15 while performing position verifications with the CamScale didn't affect the system calibration over time and no clear pattern of deviation from target positions was observed along multiple measurements for either channels. The maximum deviation from target position for channels 1 and 15 was $0.5 \mathrm{~mm}$ for $0.3 \%$ of the measurements with the source cable and $0.6 \mathrm{~mm}$ for $1.4 \%$ of the measurements with the dummy cable. A systematic cable position shift of $(0.2 \pm 0.1) \mathrm{mm}$ was observed between channels 1 and 15 for both source and dummy cables (positions measured with channel 15 were more distal), which is in agreement with results shown in Figure 5.20.

\subsubsection{CamScale behavior when not in reference position}

The maximum deviation from target position using channels 1 and 15 with the CamScale in non-reference positions was $0.4 \mathrm{~mm}$ for the source cable and $0.7 \mathrm{~mm}$ for the dummy cable. Such deviations are within the same range observed when cycling through the channels with the CamScale in reference position (Figure 5.20). All reported deviations were within manufacturer tolerance of $1 \mathrm{~mm}$.

\subsubsection{Source behavior verification inside a QA device}

\subsubsection{Dwell Positions and inter-dwell distances (IDDs)}

All reported uncertainties related to source positions are only from standard deviations over multiple measurements, the precision while tracking the source position is $0.1 \mathrm{~mm}$ 
for IDDs ranging from 1 to $30 \mathrm{~mm}$ and $0.5 \mathrm{~mm}$ for IDD of $100 \mathrm{~mm}$. Good agreement was observed between measured and planned IDDs. Measured IDDs for planned IDDs of $1,3,5,10,20,25,30$ and $100 \mathrm{~mm}$ were, respectively, $(1.0 \pm 0.1),(3.0 \pm 0.1),(5.0 \pm 0.1)$, (10.0 \pm 0.1$),(20.0 \pm 0.1),(25.0 \pm 0.1),(29.9 \pm 0.2),(99.3 \pm 0.1) \mathrm{mm}$. The greater deviation between measured and planned IDDs for planned IDDs of 30 and $100 \mathrm{~mm}$ is due to greater experimental uncertainty as the high-speed camera field of view and, consequently, pixel size is increased.

When recording both dummy and source cable movements for a single planned dwell position $1 \mathrm{~mm}$ away from the tip, the measured position for the dummy cable was $(0.9$ $\pm 0.1) \mathrm{mm}$ from the tip, while the source cable briefly ( $\sim 0.1$ second $)$ overshoots the target position, stopping at $(0.8 \pm 0.1) \mathrm{mm}$ and then moves to the dwell position $(1.2 \pm 0.1) \mathrm{mm}$ from the tip. This behavior of the source cable was present for all plans evaluated and can be seen on supplementary video $2^{3}$, which shows the source movement for a plan with IDD $=10 \mathrm{~mm}$ and planned dwell time of 1 second. This small deviation $(\sim 0.2 \mathrm{~mm})$ for the first dwell position of the source cable shifts the remaining dwell positions, causing an average negative deviation from target positions, as shown in Figure 5.21, where deviations are grouped by IDD for all the plans with dwell times of 1 second. All measured deviations from planned dwell positions are within the manufacturer tolerance of $1 \mathrm{~mm}$.

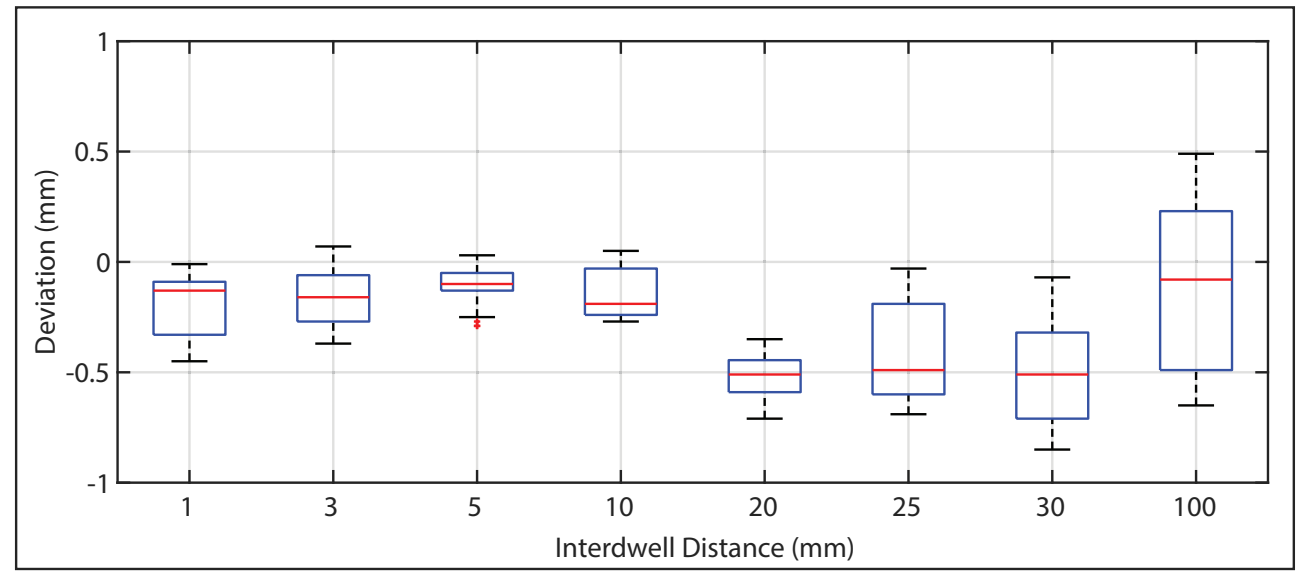

Figure 5.21. Deviations between measured and planned absolute dwell positions related to the tip of the transparent QA device. Deviations (grouped by IDD) were obtained by comparison between high-speed camera measurements and the post irradiation report emitted by the afterloader.

The precision and accuracy of dwell positions for plans with offsets or distal corrections applied were evaluated with IDD of $5 \mathrm{~mm}$, dwell time of 0.2 seconds and flexible applicator settings. Results are shown in Table 5.4. All measurements showed a mean deviation

\footnotetext{
${ }^{3}$ Available at: https:// doi.org/10.1016/j.brachy.2019.06.005
} 
from planned dwell positions below $0.35 \mathrm{~mm}$, the maximum deviation was $-0.49 \mathrm{~mm}$ and all dwell positions were within a range of $0.50 \mathrm{~mm}$ from planned positions. Deviations measured for the first dwell positions are equivalent to deviations for all dwell positions considering the uncertainties, indicating that dwell positions of the same plan were mostly shifted, but presented a small IDD variation. Deviations for plans with offsets or distal corrections applied are within the same range and according to manufacturer tolerances, resulting same practical effect

Table 5.4. Deviation between measured and planned absolute dwell positions related to the tip of the transparent QA device for plans with IDD of $5 \mathrm{~mm}$, dwell time of $0.2 \mathrm{sec}$, flexible applicator settings with offset or distal corrections applied. Deviations for the first dwell position are highlighted in a separate column.

\begin{tabular}{cc|cc}
\hline \multirow{2}{*}{ Experimental settings } & \multicolumn{2}{|c}{$\begin{array}{c}\text { Deviation between measured and planned positions } \\
\text { Mean (range) }(\mathrm{mm})\end{array}$} \\
\cline { 3 - 4 } & 0 & First Dwell Position & All Dwell Positions \\
\hline \multirow{4}{*}{ Offset $(\mathrm{mm})$} & 1 & $-0.22(-0.35: 0.01)$ & $-0.28(-0.49: 0.01)$ \\
& 2 & $0.01(-0.31: 0.09)$ & $-0.22(-0.41: 0.09)$ \\
& 4 & $-0.19(-0.33:-0.01)$ & $-0.07(-0.35: 0.09)$ \\
& 10 & $-0.06(-0.29: 0.07)$ & $-0.27(-0.47:-0.01)$ \\
\multirow{4}{*}{ Distal correction $(\mathrm{mm})$} & 1 & $-0.26(-0.31:-0.17)$ & $-0.33(-0.33: 0.07)$ \\
\hline & 2 & $-0.12(-0.19:-0.05)$ & $-0.16(-0.29:-0.05)$ \\
& 4 & $-0.20(-0.25:-0.15)$ & $-0.25(-0.31:-0.15)$ \\
& 10 & $-0.08(-0.13:-0.03)$ & $-0.09(-0.15:-0.03)$ \\
\hline
\end{tabular}

Measurements performed using the QA device for plans with rigid and flexible applicator settings didn't affect IDD values, being $(5.0 \pm 0.1) \mathrm{mm}$ in both cases. Nevertheless, the dwell positions were shifted due to deviations in the distal position. The mean deviation for the first dwell position was $(0.1 \pm 0.3) \mathrm{mm}$ with rigid applicator settings and ($0.2 \pm 0.2) \mathrm{mm}$ with flexible applicator settings. All deviations are within manufacturer tolerances. The shift in the dwell positions, however, is believed to be due to differences in length verification when a push test is performed for rigid applicators. Considering the uncertainties, these values are equivalent, nonetheless. Length verification deviations between rigid and flexible applicators is further discussed in subsection 5.5.3. A change in channel length due to temperature variations over multiple measurements was not detected experimentally at room temperature $\left(23-26^{\circ} \mathrm{C}\right)$.

\subsubsection{Transit time and Dwell time evaluation}

Measured transit time for the first dwell position was always equal or greater than the transit time for the remaining dwell positions (Table 5.5). Measured transit times were lower than minimum allowed dwell times for all cases evaluated, except for the first dwell 
Table 5.5. Minimum dwell time allowed by the Bravos system and measured transit time for the first and remaining dwell positions of plans with IDD ranging from 1 to $100 \mathrm{~mm}$ with first dwell position at the tip of the channel.

\begin{tabular}{cccc}
\hline $\begin{array}{c}\text { IDD } \\
(\mathrm{mm})\end{array}$ & $\begin{array}{c}\text { Min. dwell time } \\
\text { allowed }(\mathrm{sec})\end{array}$ & $\begin{array}{c}\text { Transit Time for the 1st dwell } \\
\text { Mean (range) }(\mathrm{sec})\end{array}$ & $\begin{array}{c}\text { Transit Time remaining dwells } \\
\text { Mean (range) }(\mathrm{sec})\end{array}$ \\
\hline 1 & 0.10 & $0.12(0.11: 0.12)$ & $0.03(0.02: 0.04)$ \\
3 & 0.15 & $0.15(0.14: 0.17)$ & $0.08(0.07: 0.10)$ \\
5 & 0.20 & $0.17(0.17: 0.19)$ & $0.11(0.10: 0.13)$ \\
10 & 0.25 & $0.22(0.21: 0.24)$ & $0.19(0.17: 0.20)$ \\
20 & 0.30 & $0.29(0.28: 0.30)$ & $0.27(0.25: 0.30)$ \\
25 & 0.35 & $0.32(0.31: 0.33)$ & $0.32(0.31: 0.33)$ \\
30 & 0.40 & $0.34(0.33: 0.35)$ & $0.34(0.31: 0.36)$ \\
100 & 0.40 & $0.34(0.33: 0.35)$ & $0.34(0.30: 0.40)$ \\
\hline
\end{tabular}

position of plans with dwell times of 0.10 and $0.15 \mathrm{sec}$, meaning that for these specific cases the real dwell time will unavoidably exceed planned dwell times by at least 0.02 second.

Bravos system calculates the transit time considering a maximum region within $15 \mathrm{~mm}$ from the dwell position (see section 4.6), meaning that the transit time for IDDs greater than $30 \mathrm{~mm}$ or single dwell positions are considered the same, measured as $0.35 \pm 0.05$ second (Table 5.5). Doses measured by J. Jeong et al.[134] using the GammaMedplus iX afterloader for a single dwell position with and without transit dose resulted in an equivalent transit time of $0.84 \pm 0.09$ second. Such correction was adopted by Bellezzo et al.[76] with good agreements. This difference in equivalent transit times is because J. Jeong et. al. measurements considered the whole source path with the purpose of correcting the dose at one dwell position while the transit time considered by the Bravos system has the purpose of correcting the dose locally with minimum interference with the whole dose distribution.

Dwell times for the first dwell positions were also evaluated separately from the others due to the difference in transit time shown in Table 5.5. Good agreement between measured and reported dwell times was observed. Measured dwell times for the first dwell position were, on average, 0.01 seconds less than reported by the Bravos system (maximum deviation of 0.02 second) and 0.02 seconds greater than planned (maximum deviation of 0.07 second). For the remaining dwell positions, average deviations between planned, measured and reported dwell times were lower than $0.01 \mathrm{sec}$, with maximum deviation of 0.02 seconds between measured and reported dwell times, and 0.04 seconds between measured and planned.

The Bravos system not only detects when delivered dwell times are different than planned (dwell time accuracy of \pm 0.05 seconds [150]) but also compensates this difference by 
changing delivered dwell time for the closest dwell positions, aiming to achieve an average irradiation time according to plan. For the most critical case observed (IDD $=1 \mathrm{~mm}$ and 0.10 seconds dwell time) the delivered dwell time reported by the afterloader for the first dwell position was 0.09 seconds greater than planned, however the next six dwell positions had a delivered dwell time lower than planned, making the average deviation from planned dwell times considering all dwell positions less than 0.001 second.

For plans in which transit time correction was disabled (only possible for test plans created using physics mode) the irradiation time unavoidably exceed the planned time, nevertheless, the mean difference between measured and reported dwell times was less than 0.001 second.

\subsubsection{Speed profiles and acceleration}

Speed profiles and acceleration were evaluated only for the source cable, the time required to perform dummy checks was not evaluated. The source cable moves in a similar fashion for the Bravos and GammaMedplus iX afterloaders; in a uniformly accelerated movement ( $|\mathrm{a}|$ measured as $(152 \pm 4) \mathrm{cm} / \mathrm{s}^{2}$ for the Bravos and reported as $154 \mathrm{~cm} / \mathrm{s}^{2}$ [79] for the GammaMedplus iX) until reaching the mid-distance between dwell positions or achieving a maximum speed ${ }^{4}$ of $100 \mathrm{~cm} / \mathrm{s}$ [26] for the Bravos system and $63 \mathrm{~cm} / \mathrm{s}$ [79] for the GammaMedPlus iX. Bravos source acceleration is greater than reported for other afterloaders such as the Nucletron microSelectron v.3 $\left(113 \mathrm{~cm} / \mathrm{s}^{2}\right.$ [82]), Nucletron Oldelf $\left(78 \mathrm{~cm} / \mathrm{s}^{2}\right.$ [168]) and the GammaMed $12 \mathrm{i}\left(55 \mathrm{~cm} / \mathrm{s}^{2}\right.$ [168]), although different values have been reported for identical afterloader models in different studies (not related to the Bravos afterloader), as summarized by Fonseca et. al. [30, 122]. Table 5.6 shows the maximum and average source speeds for different IDD for the Bravos afterloader. Results agree within combined uncertainties. Source speeds values for the Bravos afterloader are lower than measured by Fonseca et. al. [30] for the Nucletron microSelectron v.3 afterloader and approximately twice as fast as measured by Wojcicka et. al. [168] for the GammaMed 12i afterloader.

\subsubsection{Source behavior verification inside clinical applicators}

\section{Cable snaking and first dwell position}

The cable snaking effect caused when the source cable is curled inside the catheter when it is pushed against the end of the applicator isn't present with the Bravos system due to the pre-treatment length verification combined with the restriction of not having the first (most distal) dwell position closer than $1 \mathrm{~mm}$ to the tip of the applicator. The mean

\footnotetext{
${ }^{4}$ Maximum speed was not reached during the experiments.
} 
Table 5.6. Maximum and average source speed while moving between two consecutive dwell positions inside the QA device for IDD ranging from 1 to $100 \mathrm{~mm}$. Speeds were measured with a high-speed camera and calculated considering a uniformly accelerated movement with $|\mathrm{a}|=(152 \pm$ 4) $\mathrm{cm} / \mathrm{s}^{2}$

\begin{tabular}{ccccc}
\hline IDD & \multicolumn{2}{c}{ Average Speed $(\mathrm{cm} / \mathrm{s})$} & \multicolumn{2}{c}{ Maximum Speed $(\mathrm{cm} / \mathrm{s})$} \\
$(\mathrm{mm})$ & Measured & Calculated & Measured & Calculated \\
\hline 1 & $1.8 \pm 0.2$ & $1.9 \pm 0.1$ & $3.2 \pm 0.4$ & $3.9 \pm 0.1$ \\
3 & $3.4 \pm 0.2$ & $3.4 \pm 0.1$ & $6.2 \pm 0.3$ & $6.7 \pm 0.1$ \\
5 & $4.3 \pm 0.2$ & $4.4 \pm 0.1$ & $8.3 \pm 0.5$ & $8.7 \pm 0.1$ \\
10 & $6.0 \pm 0.3$ & $6.2 \pm 0.1$ & $11.9 \pm 0.4$ & $12.3 \pm 0.2$ \\
20 & $8.7 \pm 0.3$ & $8.7 \pm 0.1$ & $16.9 \pm 0.4$ & $17.4 \pm 0.3$ \\
25 & $9.5 \pm 0.5$ & $9.8 \pm 0.2$ & $19.0 \pm 0.4$ & $19.0 \pm 0.3$ \\
30 & $10.6 \pm 0.4$ & $10.7 \pm 0.2$ & $20.8 \pm 0.8$ & $21.3 \pm 0.3$ \\
100 & $19.8 \pm 0.4$ & $19.5 \pm 0.3$ & $38.6 \pm 0.6$ & $39.0 \pm 0.5$ \\
\hline
\end{tabular}

deviation from target position for the first dwell position considering all plans for rigid applicators without offset was $(0.1 \pm 0.3) \mathrm{mm}$, with maximum deviation of $0.6 \mathrm{~mm}$ for $2 \%$ of the positions evaluated. Table 5.7 shows the average deviation from the first dwell position for each applicator evaluated.

\section{Source path and IDD}

For curved applicators, the source cable follows a longer path while moving from the afterloader to the first dwell position (following the outer wall of the channel) than while moving from the first dwell position to the remaining positions (following the inner wall of the channel). Only part of the retraction length causes a change in source position when the cable is retracted from distal dwell positions, while the remaining retraction length tightens the cable to a shorter path. The Bravos system extends the cable past the first dwell position to be able to tighten the cable when retracting it to the first dwell position. This correction may not be enough depending on the difference in path length of each applicator, resulting in shorter IDDs between first and second dwell positions. Such variations should be evaluated during applicator commissioning. Table 5.7 shows the average deviation from planned dwell position for the first $45 \mathrm{~mm}$ of each applicator evaluated for IDDs of 2, 5 and $10 \mathrm{~mm}$, and the first $20 \mathrm{~mm}$ for IDD of $1 \mathrm{~mm}$. For the 45 $\mathrm{mm}$ range, all applicators, except the ring, present an average deviation lower than 1.0 $\mathrm{mm}$ with local deviations of up to $1.0 \mathrm{~mm}$ for the Colpostat and Tandem 15 applicators. Deviations closer to the tip are highlighted for a $20 \mathrm{~mm}$ range and $1 \mathrm{~mm}$ IDD, resulting in average deviations greater than $1 \mathrm{~mm}$ for the Colpostat and Ring applicators, which are the two applicators with most accentuated curvatures.

Due to its accentuated curvature, the ring applicator has average deviations from planned positions ranging from 1.3 to $2.5 \mathrm{~mm}$, whilst average deviation reported in the literature 
Table 5.7. Average deviation between planned and measured dwell positions for the first (most distal) dwell position (results for IDD = 2, 5 and $10 \mathrm{~mm}$ combined) and for all dwell positions for a range of $45 \mathrm{~mm}$ starting at the tip of the applicator for IDDs of 2, 5 and $10 \mathrm{~mm}$ and a range of 20 $\mathrm{mm}$ for IDD of $1 \mathrm{~mm}$

\begin{tabular}{cc|cccc}
\hline \multirow{2}{*}{ Applicator } & First dwell position & \multicolumn{4}{|c}{ Average Deviation from Target position } \\
& IDD $=2,5,10 \mathrm{~mm}$ & IDD $=1 \mathrm{~mm}^{*}$ & IDD $=2 \mathrm{~mm}$ & IDD $=5 \mathrm{~mm}$ & IDD $=10 \mathrm{~mm}$ \\
\hline Colpostat & $0.2(-0.1: 0.5)$ & $-1.2(-1.5:-0.4)$ & $-0.3(-1.0: 0.4)$ & $-0.1(-1.0: 0.7)$ & $-0.3(-0.9: 0.1)$ \\
Needle & $0.1(-0.2: 0.4)$ & $0.1(0.0: 0.3)$ & $0.1(-0.4: 0.7)$ & $0.1(-0.2: 0.6)$ & $0.4(0.1: 0.6)$ \\
Ring & $-0.1(-0.3: 0.1)$ & $-2.5(-3.2:-0.3)$ & $-1.8(-3.0: 0.1)$ & $-1.6(-2.6: 0.1)$ & $-1.3(-2.3: 0.1)$ \\
Tandem 15 & $0.2(-0.1: 0.6)$ & $-0.3(-0.6: 0.5)$ & $-0.6(-1.0: 0.3)$ & $-0.4(-0.9: 0.5)$ & $-0.1(-0.6: 0.6)$ \\
Tandem 45 & $0.0(-0.3: 0.3)$ & $-0.8(-1.1:-0.3)$ & $-0.2(-0.9: 0.3)$ & $-0.1(-0.8: 0.3)$ & $-0.2(-0.6: 0.2)$ \\
Tandem Ring & $0.3(-0.1: 0.5)$ & $-0.4(-0.7:-0.2)$ & $0.2(-0.5: 0.7)$ & $0.3(-0.4: 0.7)$ & $0.2(-0.3: 0.6)$ \\
\hline
\end{tabular}

*Evaluated range of $20 \mathrm{~mm}$

are $(2.5-4.5 \mathrm{~mm})[77,164]$ when no offset correction is applied. The maximum local deviation found in this study was $3.2 \mathrm{~mm}$. While deviations of up to $6.1 \mathrm{~mm}$ (also without offset correction) are reported by Awunor et al.[169], suggesting that the length verification feature of the Bravos system mitigates such deviations. Both local and average deviations can be reduced if dwell positions are purposely shifted from planned positions to compensate for the first few smaller IDDs, increasing the deviation for the distal position but reducing the deviation for the remaining ones. This effect can be achieved if a distal correction is applied during the pre-treatment verification or by applying an offset to the original plan (see Figure 5.22). For a plan with IDD of $5 \mathrm{~mm}$ the initial deviation is -1.6 (-2.6: 0.1$) \mathrm{mm}$ (mean (range), from Table 9), however, this deviation changes to -0.4 $(-1.6: 1.2) \mathrm{mm}$ if $1 \mathrm{~mm}$ of distal correction is applied and $0.5(-0.6: 2.2) \mathrm{mm}$ for $2 \mathrm{~mm}$ of distal correction.

Applying a distal correction on the ring applicator improves the average deviation from planned positions for the region evaluated. Nevertheless, the deviation between measured and planned dwell positions changes along the channel while the cable isn't completely tightened, and different distal correction values may result in lower average deviation if different regions are evaluated. Considering the same IDD $(5 \mathrm{~mm})$ and dwell positions region ( $45 \mathrm{~mm}$ from the tip), distal corrections didn't improve the average deviation from planned positions for the remaining applicators, since all of them had an average deviation greater than $-0.5 \mathrm{~mm}$ and the minimum distal correction possible is of $1 \mathrm{~mm}$. A proper applicator commissioning is crucial to define the best protocol to be adopted for each applicator, however this is out of the scope of this study.

\section{Flexible vs rigid applicator}

The force threshold used to define the channel length for flexible applicators is lower than for rigid applicators to avoid damaging the tip of a flexible applicator. The lower force 


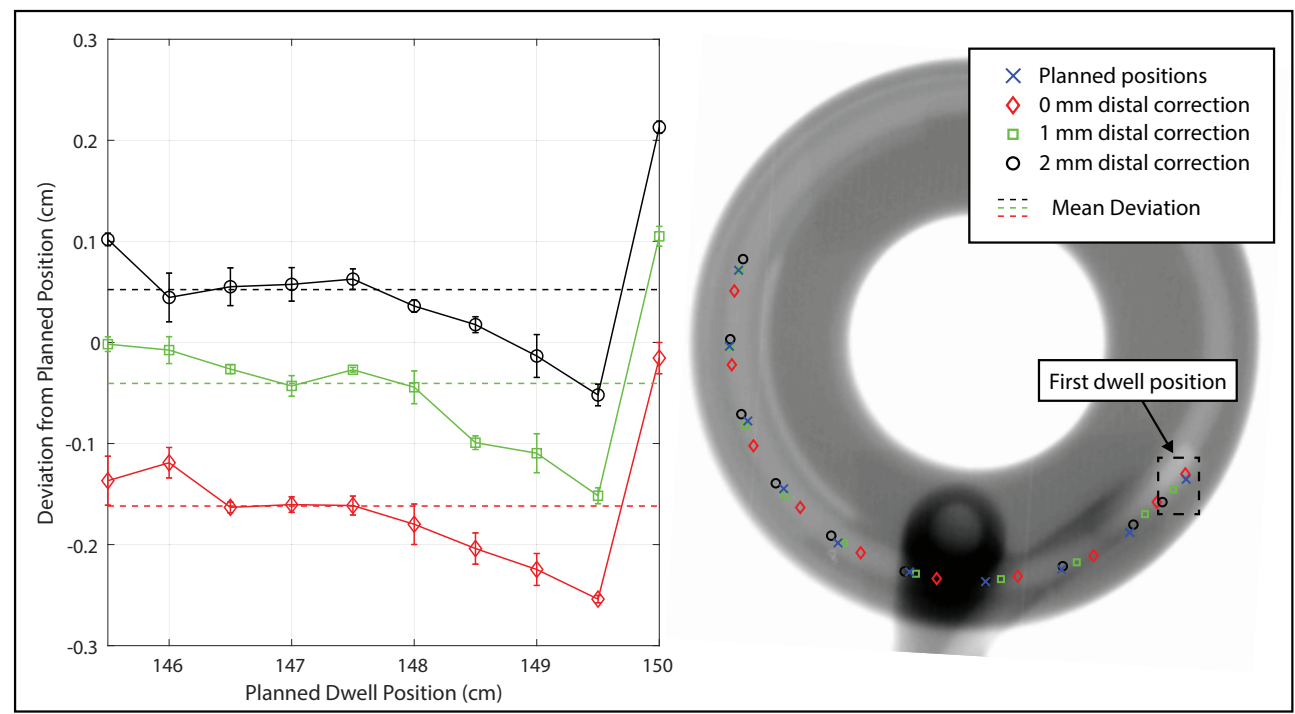

Figure 5.22. Difference between planned and measured dwell positions for the ring applicator when distal corrections of 0,1 and $2 \mathrm{~mm}$ are applied to shift the dwell positions and compensate for the smaller IDDs close to the end of the channel. Continuous lines show the deviation for each dwell position while dashed lines show the average deviation over a range of $45 \mathrm{~mm}$.

threshold can, however, interfere with the length verification of rigid applicator if they are wrongly defined as flexible. Such error would be detected during the commissioning of the applicators and the Bravos system warns the user if measured channel length is different than planned to mitigate human errors. Nevertheless, the choice between rigid and flexible applicator settings should be adopted with caution. Figure 5.23 shows two examples of wrong length verification caused by incorrectly using flexible applicator settings for curved rigid applicators. The red arrows point where the dummy cable stopped during the length verification of each case.

\subsection{Transit time}

\subsubsection{Source movement prediction}

Figure 5.24 shows the source movement registered with the high-speed camera for the same plan executed five times using the Bravos afterloader with transit time correction enabled, against the source movement prediction for a plan with IDD of $5 \mathrm{~mm}$ and dwell time of 1 second, as an example.

Measured and predicted movements match well, however, small differences are detected. The source cable should overshoot the first dwell position $(130.0 \mathrm{~cm})$ by $1 \mathrm{~mm}$ followed by 


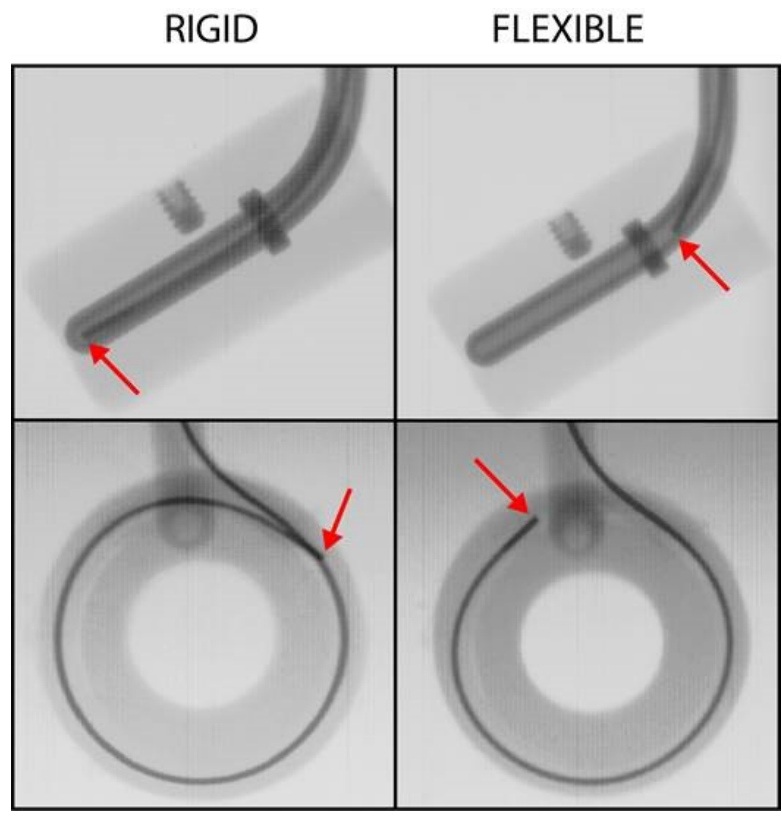

Figure 5.23. Difference in length verification when flexible applicator settings are incorrectly assigned to rigid curved applicators. The red arrows indicate where the dummy cable stops during the length verification when rigid and flexible applicator settings are adopted.

a $1 \mathrm{~mm}$ retraction to remove cable slack. However, measurements show an overshoot of less than $1 \mathrm{~mm}(\sim 0.5 \mathrm{~mm})$ with a quick pause of $\sim 0.04$ seconds. Both deviations are within manufacturer uncertainty specifications of $1.0 \mathrm{~mm}$ and 0.05 seconds [150], however, the overshoot deviation is known by the manufacturer to be due to the change in movement direction of the source cable. Therefore, an extra transit time correction is applied to the first dwell position, resulting in a dwell time (including transit time) $\sim 0.02$ seconds shorter than planned, which is also within manufacturer uncertainty specifications. This deviation on the first dwell time causes a time shift on the source movement, however the remaining dwell times are correct, with deviation lower than 0.01 seconds between measurement and prediction.

\subsubsection{Transit Dose}

Figure 5.25a shows the simulated dose profile for a plan with IDD of $10 \mathrm{~mm}$, planned dwell time of 5.0 seconds per dwell position, scored $5 \mathrm{~mm}$ away from the source axis. The source center ( $0.247 \mathrm{~mm}$ from the source tip) at each dwell position is highlighted by vertical dashed lines. Transit time correction was applied to the source movement using the Bravos and GammaMedplus iX algorithms. 


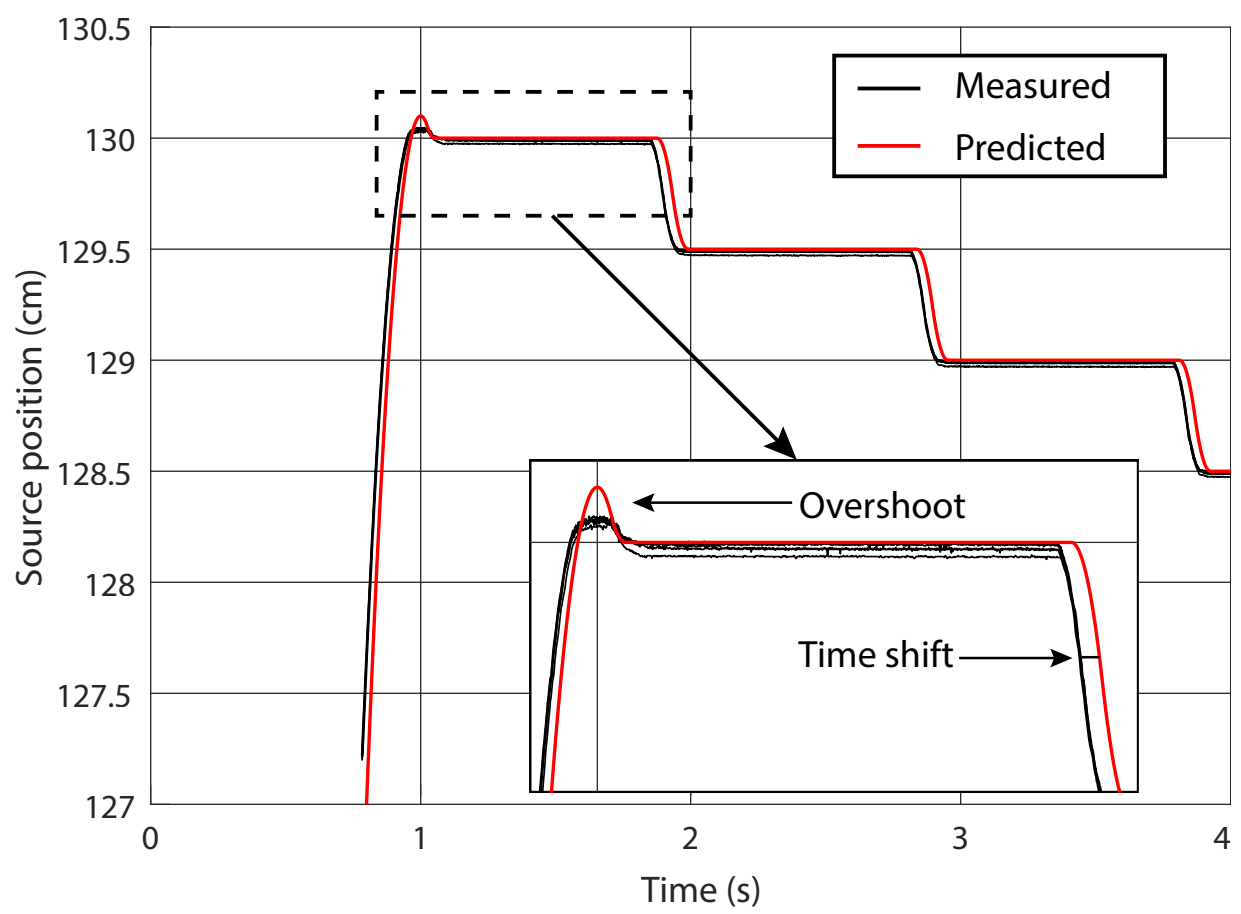

Figure 5.24. Comparison between measurement and prediction of a source movement for a plan with $5 \mathrm{~mm}$ of interdwell distances and dwell times of 1 second with transit time correction following the Bravos algorithm. The inset shows the first dwell position, with measured dwell time 0.02 seconds lower than planned, causing a time shift on the source movement profile, but with differences between measured and planned dwell times lower than 0.01 seconds for all remaining dwell positions.

Apart from the from the first and last dwell positions using the GammaMedplus iX transit time algorithm, no clear difference in dose profiles can be observed in Figure 5.25a. Nevertheless, the absolute dose deviation (Figure 5.25b) and percentage dose deviation (Figure 5.25c) between the planned dose profile and dose profiles considering the transit dose corrections show that the dose profile for the GammaMedplus iX afterloader is greater than planned with maximum absolute deviations at the first and last dwell positions, while dose profile deviations using the Bravos transit time correction algorithm oscillates close to zero, resulting in a low average dose deviation. The average transit time measured for dwell positions with IDD of $10 \mathrm{~mm}$ is $\sim 0.2$ seconds considering the Bravos algorithm (Table 5.5). Since the GammaMedPlus iX doesn't apply a transit time correction to the first and last dwell positions, the dwell time (time in dwell position + transit time) for both dwell positions would be approximately 5.2 seconds instead of 5.0 seconds, resulting in dwell time $\sim 4 \%$ greater than planned, which is in agreement with the dose difference 


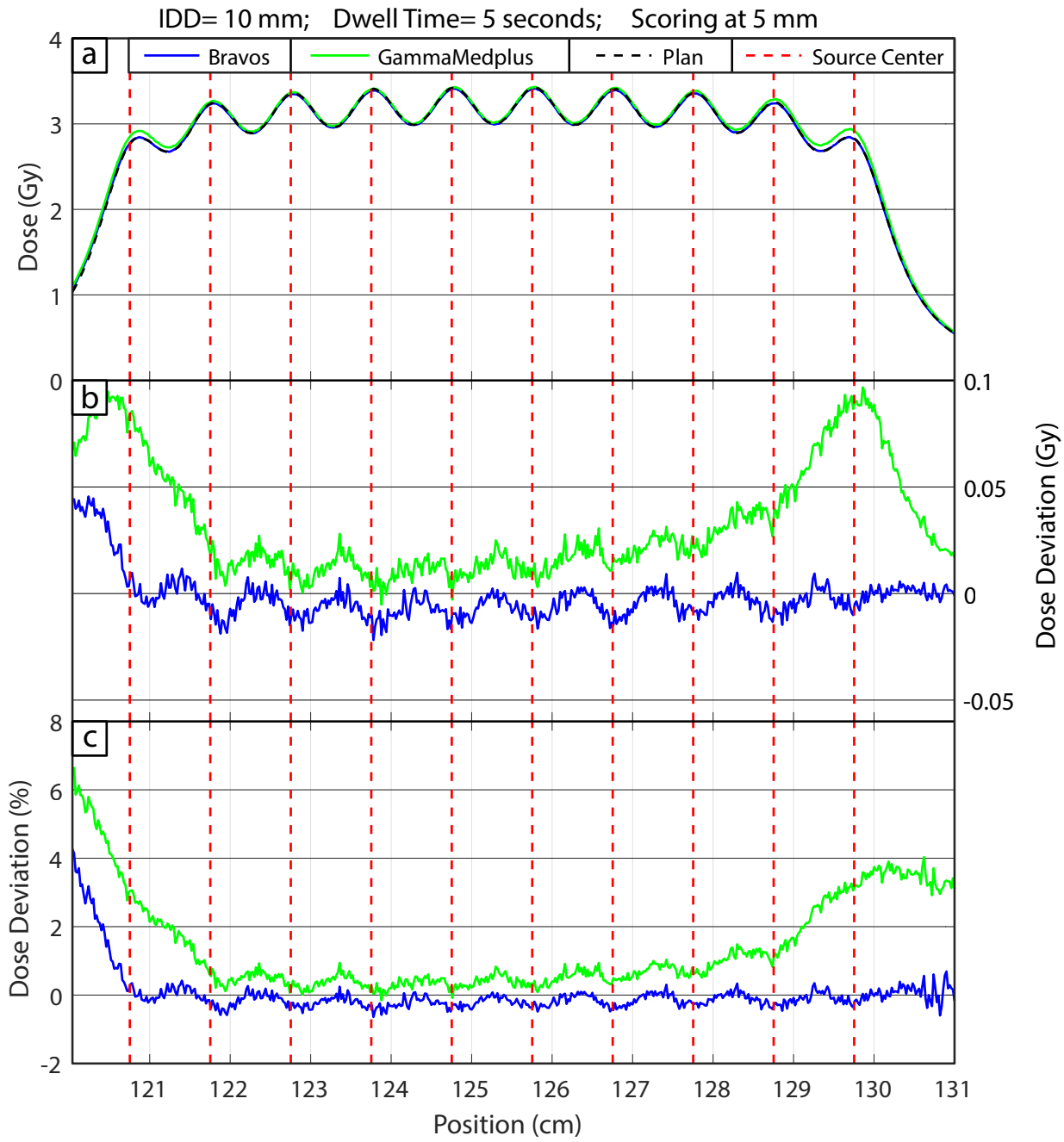

Figure 5.25. Dose profiles scored at radial distance of $5 \mathrm{~mm}$ from the source for a plan with 10 dwell positions with IDD of $10 \mathrm{~mm}$ and dwell times of 5 second. Dose profiles were simulated considering the source at each dwell position for its dwell time (no transit - Plan simulation) and considering the source movement following the Bravos and GammaMedplus iX transit time correction algorithms. Dose profiles were compared considering a) absolute dose; b) Absolute deviation from planned dose and c) Relative deviation from planned dose.

shown in Figure 5.25c due to lack of transit time correction. Moreover, Jeong et al. [134], measured a $2.8 \pm 0.3 \%$ dose increment due to the transit dose for a plan with a single dwell position with a $40700 \mathrm{U}$ source and 30 seconds of dwell time. Such correction was used by Bellezzo et al.[76], resulting in a good agreement between simulations and experimental 
results.

Since transit time corrections are always applied when the source is at its dwell position, there is a clear increase in dose deviation when the source is moving back to the afterloader due to the transit dose that is not corrected after the last dwell position, as shown in Figure $5.25 \mathrm{~b}$ and c. This dose difference, however, will quickly reduce as the source accelerates while moving back to the afterloader.

Figure 5.26 shows the maximum (Figure 5.26a) and average (Figure 5.26b) percentage dose deviation from planned dose distribution for the region between the first and last dwell positions of several plans with active length of $11 \mathrm{~cm}$. Each plan has the same planned IDD and dwell time for all dwell positions. Each marker represents a different dose profile simulation. Marker colors represent different IDDs, marker shapes represent the scoring distances, and filled markers represent simulations using the GammaMedplus iX transit time correction algorithm while the unfilled markers represent simulations using the Bravos algorithm.

Results show a greater dose deviation when using the GammaMedplus iX transit time correction algorithm, especially due to the lack of transit time correction for the distal and proximal dwell positions. Moreover, the distal dwell position is the most affected by lack of transit time correction since the source speed reduces as it approaches the distal position and overshoot it by $1 \mathrm{~mm}$. For that reason, the maximum dose deviation from planned for the GammaMedplus iX afterloader was always for the first dwell position. Nevertheless, the average dose deviation for the cases evaluated reduces to a maximum value of $6 \%$ for dwell times of 1.0 second.

The Bravos algorithm has a lower dose deviation from planned. Interestingly though, the average dose distribution is lower than planned for several cases, reaching a minimum of $-0.4 \%$ for plans with 1.0 second dwell times. As expected, longer dwell times yield lower deviations for both afterloaders, since the transit time becomes less relevant when compared to the dwell time. 


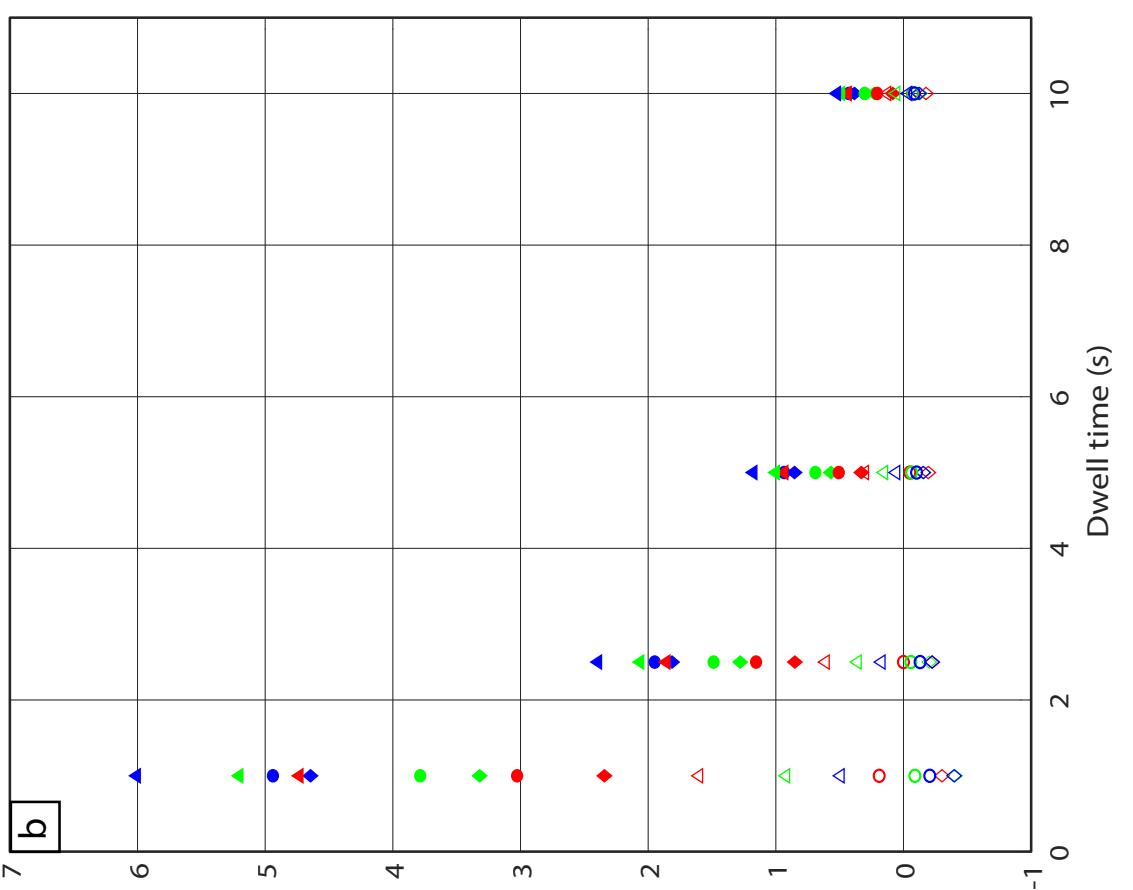

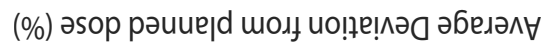

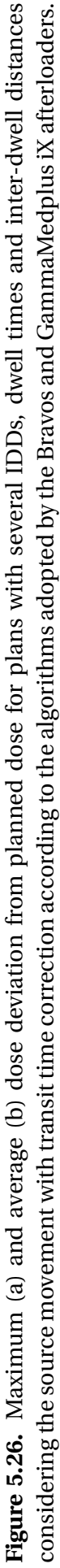




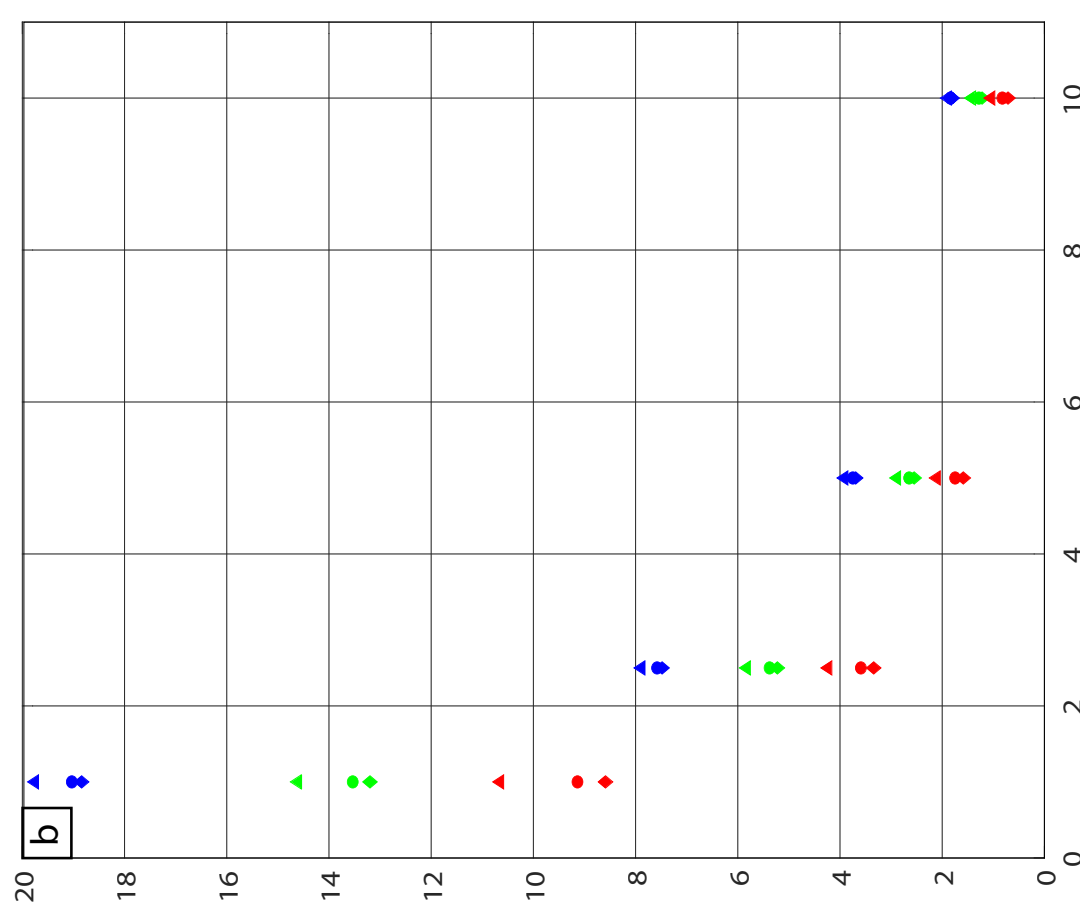

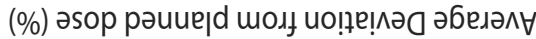

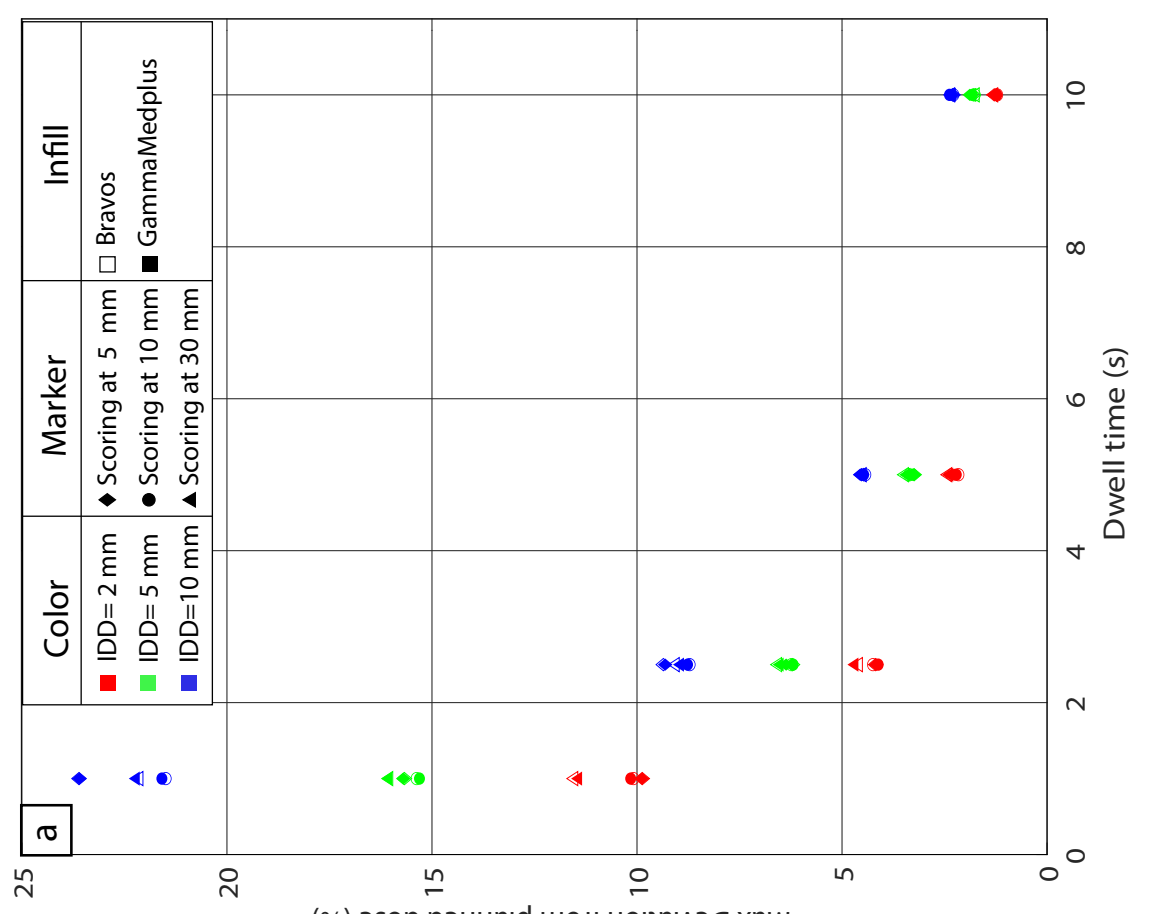

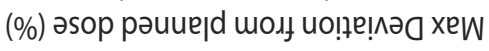


Dose deviations for plans without transit time correction present a small difference between maximum and average deviations (Figure $5.27 \mathrm{a}-\mathrm{b}$ ), and no difference between afterloaders (as anticipated, since the movement prediction without transit time correction is exactly the same for both afterloaders for the evaluated plans). Reaching maximum deviations greater than $20 \%$ for dwell times of 1.0 second and IDD of $10 \mathrm{~mm}$. Although such values seem high, they are in agreement with the transit time measured with the high-speed camera shown in Table 5.5, where the transit time for IDD of $10 \mathrm{~mm}$ is $\sim 0.2$ seconds, which is $20 \%$ of 1.0 second.

\subsubsection{Discussion}

None of the transit time correction algorithms can perfectly correct for the transit dose delivered during the source movement, which is expected since the time correction is applied on the dwell positions while the transit dose is delivered along the whole source path. The best that a transit time correction algorithm can do is to deliver an average irradiation time close to planned, but with local deviations along the source path, essentially reducing the dwell times at the dwell positions to compensate for the extra dose already delivered during the source movement. Nevertheless, results show that transit time correction is increasingly important as dwell times are reduced. Moreover, the Bravos algorithm shows a substantial improvement when compared to the GammaMedplus iX algorithm, with 35 out of 36 plans evaluated with average deviation from planned dose distribution lower than $1 \%$ while the GammaMedplus iX algorithm has 17 plans for which the dose deviation was lower than $1 \%$. Moreover, the maximum local deviation using the Bravos algorithm was 3.5\% for a single plan and all remaining plans with deviations lower than $3.0 \%$, while the GammaMedplus iX algorithm had 15 plans with local deviation greater than $3 \%$, reaching a maximum deviation of $16.6 \%$.

Such improvement of the Bravos algorithm is due to two main reasons:

1) considering the transit dose delivered when the source is moving from the afterloader to the most distal position;

2) applying a transit time correction to the first and last dwell positions.

Due to the greater source speed, the transit time component resulting from the source movement from the afterloader to the distal dwell position has a lower impact to the total transit time than the transit time component when the source is moving between dwell positions, as illustrated in Figure 4.13b. However, its effect is increasingly important as the source speed reduces while approaching the distal dwell position. Furthermore, the transit time correction for the first dwell position has a big effect on local dose distribution, as shown in Figure 5.25c and Figure 5.26, where the lack of transit time correction for the 
first and last dwell positions results in local deviations of up to $16.5 \%$ for plans with dwell times of 1.0 second.

Regardless of the algorithm used for transit time correction, results show a greater average dose deviation for lower dwell times. For that reason, dwell times lower than 0.5 seconds are not used in some clinics $[30,122]$, and modern afterloaders have a minimum allowed dwell time depending on the IDD due to the transit dose [25], as shown in Table 5.5.

The focus of this study was on the impact that a different transit time correction algorithm would have on the dose distribution and, for that reason, only plans for which both afterloaders would have the same source movement (without transit time correction) were evaluated. However, another important factor not directly related to the transit time correction algorithms, but with impact in the dose distribution is that the Bravos afterloader extends the source until the most distal position but no further, while the GammaMedplus iX afterloader always extends the source until the end of the channel and then retracts it to the distal position. This behavior will increase even more the transit dose to the first dwell position for plans in which an offset is applied.

Fonseca et al. evaluated the transit dose contribution for gynecological and prostate cases considering speed profiles available in the literature [122]. The afterloader with the closest acceleration to the Bravos afterloader was the MicroSelectron (Elekta Stockholm, SE) with acceleration of $113 \mathrm{~cm} / \mathrm{s}^{2}$, resulting in transit dose contributions between 0.5 and $1.8 \%$ for gynecological cases evaluated, and between 3.8 and $11.1 \%$ for prostate cases. The transit dose contribution to the total dose changes according to the applicator used, channel arrangement, dwell times and inter-dwell distances. Figure 5.1a shows that prostate treatments typically have lower dwell times than gynecological treatments. Therefore, a greater transit dose contribution for prostate cases is expected and it is in agreement with results shown in Figure 5.26. 

6

\section{Conclusions}



The MAASTRO applicator was specifically designed to deliver a high surface dose ( $>30 \mathrm{~Gy}$ ) in less than 10 minutes (even with a low activity source 20350U) to a small target region of $\sim 20 \times 20 \mathrm{~mm}^{2}$ while sparing OARs surrounding the applicator, which is an advantage to EBRT boosting techniques, where the high dose region is not as localized, resulting in greater toxicity. Such dose distribution required a complex multichannel arrangement bringing the source close and parallel to the treatment surface while OARs are protected by lateral shielding. Differently than most BT applicators, the MAASTRO applicator uses the inverse square law to achieve a steep dose falloff by bringing the source in close contact to the tumor in multiple points, whilst CXB devices, such as the Papillon 50 and the Xoft eBT rectal applicator [170], rely on $50 \mathrm{kVp}$ x-rays to achieve a similar result. Nevertheless, the MAASTRO applicator was developed to be used with commonly available HDR ${ }^{192} \mathrm{Ir}$ BT sources, which allows it to be used with regular HDR afterloaders already available in most oncology centers without the need of extra equipment, differently from CXB devices that require a $50 \mathrm{kVp}$ x-rays source. For this reason, the MAASTRO applicator holds the potential of being a cost-effective solution for endoluminal boosting in radiotherapy. Furthermore, the applicator is compatible with currently available TPSs, increasing the deg of freedom to generate a patient oriented dose distribution.

It is important to point out that the MAASTRO applicator (currently going through CE labeling) could only be modeled by a multidisciplinary team, capable of finding a balance between the dose distribution requirements, geometrical limitations (for patient comfort), and manufacturing limitations.

The studies performed in this thesis adopted several non-standard techniques aiming to better understand the applicator design and dose delivery limitations, but also to improve commissioning, QA and treatment delivery in brachytherapy.

During the pretreatment verification system study, it was shown that even though the use of IPs is not common in brachytherapy, it provides considerable advantages. The performed calibration requires only an ${ }^{192} \mathrm{Ir}$ source and an afterloader, which can be an advantage for small brachytherapy centers. In this study, the use of a robotic arm and ultrasound sensors was essential to assure a high precision and accuracy on the source position with respect to the IP. The cost of the robotic arm used should not be an impediment for a radiotherapy department. However, even though the process can be automated, the software to control the robot was developed in-house and there is no software commercially available to perform such tasks, therefore someone in the radiotherapy department would have to be trained, which might be time consuming. One could consider performing the measurements in air (instead of a water tank) or use calibration phantoms. However, the study for clinical implementation is beyond the scope 
of this thesis, but subject of further research. For that reason, our research group recently received a substantial grant to work on this topic for the next 5 years.

It was demonstrated that the IP used for the treatment verification system (XRD 1640 AG9 ES - Perkin Elmer, Waltham, MA) could accurately measure Sk, dwell times and dwell positions. Therefore, all the relevant parameters of the treatment plan can be verified before delivering the treatment. Even small differences ( $1 \mathrm{~mm}$ (dwell position), 0.2 seconds (dwell time) and $1 \%$ (Sk)) could be identified. The IP employed in this study is suitable for the pretreatment verification except for the acquisition rate (7.14 fps) that was unable to measure the minimum dwell time possible ( 0.1 seconds). Ideally, an IP should acquire more than $20 \mathrm{fps}$ to be able to measure 0.1 seconds dwell times. In addition, the IP can have a smaller sensitive region since a $\sim 20 \times 20 \mathrm{~cm}^{2}$ region (instead of $40.96 \times 40.96$ $\mathrm{cm}^{2}$ ), that would be enough to verify brachytherapy treatment plans and could potentially reduce the cost of the equipment if IPs were produced in large scale.

Performing pretreatment verifications can avoid errors during the treatment, which may be highly beneficial to the patient. A reliable and detailed brachytherapy pre-treatment verification method is not commonly available due to the limitations of the currently used methods, which can lead to high uncertainties and inconclusive results. The proposed system (IP + Robotic Arm + in-house software) may overcome these technical challenges. In addition, it shouldn't be a heavy burden on the medical workflow since a large part of the measurements (image registration and positioning) and part of the data analysis can be automated.

A different IP (PaxScan 2530 HE Varex), with higher frame rate (up to $33 \mathrm{fps}$ ) and lower pixel size $(0.14 \mathrm{~mm})$, was used to increase dwell time and dwell position detection precision on the Iridium Imaging System (IrIS), which used the same principle adopted by the pretreatment verification system to track the source movement inside an opaque applicator, enabling time-resolved 2D applicator commissioning. The IrIS system is totally automated, radiopaque markers on the baseplate are used for image registration, superimposing the commissioning treatment plan from the TPS on top of the applicator projection and measuring deviations in dwell time and dwell positions in real time while the plan is delivered, which also allows the operator to adjust the source path in the TPS in order to follow the real source trajectory and repeat the measurement until dwell position deviations are minimized.

The time-resolved source position verification and the possibility of multiple measurements (without having to go inside the brachytherapy room to replace the radiochromic film) drastically increases the capabilities of detecting problems with the applicator or afterloader, especially for errors that rarely happen and are only detected after several mea- 
surements. For the example case shown in section 5.2, each one of the 19 dwell positions were measured 3 times, resulting in a total of 57 dwell points measurements. However, the system was also used in section 5.4, in which a total of 250 dwell points were evaluated in less than an hour.

The mechanical evaluation of the Bravos system showed that it has several features that differ from its predecessor, the GammaMedplus iX, with the purpose of making the treatment workflow faster and less prone to human errors, increasing the system precision during treatment delivery. The Bravos system uses the same source as the GammaMedplus iX, and the same source acceleration of $\sim 154 \mathrm{~cm} / \mathrm{s}^{2}$. Nevertheless, the source top speed was increased from $63 \mathrm{~cm} / \mathrm{s}$ to $100 \mathrm{~cm} / \mathrm{s}$, which reduces transit time and overall treatment time. Treatment time is also reduced by performing channel obstruction verification, once during the pre-treatment stage and once during treatment instead of twice during treatment. By introducing the possibility of using transfer guide tubes of different lengths, the Bravos system also implemented the length verification feature. Originally developed to verify if the connected channel has the correct length, the feature also enables a fine tuning on the dwell positions correcting them considering the measured length. Such feature indirectly corrects for differences in channel length due to transfer guide tube curvatures.

The CamScale device makes daily QA and system recalibration easier, by using video cameras to verify the source deviation from 3 pre-defined positions $(90,120$ and $150 \mathrm{~cm})$. Unfortunately, the video cameras don't record the source movement, they are only used to take a snapshot of the source when it is parked on the pre-defined positions. The cameras show the source movement in real time (without recording) at a frame rate of $25 \mathrm{fps}$, which is high enough to verify dwell times and average source speeds. The CamScale would be a more complete QA system if such features were implemented. Regardless, the CamScale is a robust and precise tool for daily QA. Deviations from target position for the source cable were below $0.5 \mathrm{~mm}$ for all measurements performed with the CamScale, even for tests with the CamScale purposely placed in the wrong position. The dummy cable position, however, was less stable than the source cable (as shown in Figure 5.20). Nevertheless, the maximum measured deviation was $0.7 \mathrm{~mm}$ for all evaluated cases, which is still within manufacturer tolerance of $1.0 \mathrm{~mm}$.

Measurements with a High Speed Camera showed that the Bravos system can accurately report delivered dwell times and deviations from planned dwell times (if any) within its accuracy of 0.05 seconds.

The most critical case evaluated (regarding deviations between planned and measured dwell times) was for a plan adopting the minimum IDD allowed ( $1 \mathrm{~mm}$ ) and the minimum 
dwell time allowed for this IDD (0.1 seconds). However, the transit time measured for the first dwell position of plans with IDD of $1 \mathrm{~mm}$ is 0.12 seconds (Table 5.5), which already exceeds the planned dwell time. The Bravos system was able to detect the deviation, reporting a delivered dwell time (including transit time) of 0.19 seconds while measurements with the high speed camera resulted in a dwell time of 0.17 seconds (the difference between measured and reported dwell times is within manufacturer tolerance of 0.05 seconds). The extra dwell time delivered to the first dwell position was automatically corrected by the system by slightly reducing the dwell time of the next 6 dwell positions, resulting in an average delivered time as planned (average deviation lower than 0.001 second) but with local variations. These local variations, however, are only present for plans using the minimum dwell time allowed for IDDs of 1 and $3 \mathrm{~mm}$, causing the maximum dose for the aforementioned case to decrease from 0.263 to $0.259 \mathrm{~Gy}$ (scored at $5 \mathrm{~mm}$ for a $40700 \mathrm{U}$ source), and shifting the peak position of the dose distribution by $\sim 0.5 \mathrm{~mm}$ towards the end of the channel. Both deviations are negligible, considering that the maximum dose deviation would be of $0.004 \mathrm{~Gy}$ per channel and $0.5 \mathrm{~mm}$ is within manufacturer tolerance. Deviations between dwell times and dwell positions measured with the high speed camera and reported as delivered by the Bravos system were all within manufacturer tolerance of 0.05 seconds and $1.0 \mathrm{~mm}$.

Features implemented in the Bravos system provide accurate dwell positions, such as the pre-treatment length verification used to confirm that the correct transfer guide tube is used (which is an essential security feature since the Bravos system allows multiple channel lengths) and to correct for small length fluctuations due to transfer guide tube curvatures, the source cable overshooting the first dwell position and then being retracted to reduce cable slack in curved applicators, and the possibility to apply pre-treatment distal corrections. Nevertheless, such features are aimed to improve treatment delivery but not to replace a proper applicator commissioning. The Bravos system also offers the possibility of defining applicators as rigid or flexible during a treatment plan, allowing the use of catheters in any of the channels. However, a lower force threshold is used during the length verification of flexible applicators and, in case of curved applicators, using flexible applicator settings for a rigid applicator may result in a wrong length measurement. Therefore, the choice between rigid and flexible applicator settings must follow the applicator specifications to avoid errors.

Elfrink et. al. [78] measured $1.0 \mathrm{~mm}$ source position accuracy for $16 \mathrm{HDR}{ }^{192} \mathrm{Ir}$ afterloaders using straight applicators while the maximum and mean deviations measured in this work for the needle applicator were 0.7 and $0.1 \mathrm{~mm}$, respectively. Rickey et al.[171] reported that bias in dwell time could be up to 0.08 seconds, which is greater than measured in 
this study. These references suggest that the Bravos system provides a lower uncertainty in treatment delivery than other HDR afterloaders. Other sources of uncertainty during a brachytherapy treatment are listed by Kirisits et. al. [77], however such analysis is beyond the scope of this thesis.

The transit time correction algorithms used by the Bravos and GammaMedplus iX afterloaders adopt a different approach to determine the transit time. Whilst the GammaMedplus iX algorithm considers the transit time as the time required for the source to move from one dwell position to the next (and that is why there is no transit time correction for the first dwell position), the Bravos algorithm considers the transit time as the time that the source spends inside a certain region close to the dwell position (as explained in section 4.6), which includes the time when the source is passing over the dwell positions while moving from the afterloader to the distal dwell position. The new algorithm also considers a transit time for the first dwell position since it doesn't use a previous dwell position (that doesn't exist for the first dwell position) to calculate the transit time.

This algorithm change has a big impact on the dose delivered by the first dwell position, reducing maximum local deviations from planned dose distribution from more than $16 \%$ to less than $4 \%$ (for evaluated cases with largest deviations), as shown in Figure 5.26a. Nevertheless, the difference between dose distributions generated from both algorithms reduces after a few dwell positions, as shown in Figure 5.25. Indicating that the biggest impact in transit time correction comes from the correction on the first dwell position, while the correction due to the time that the source spends passing over the dwell positions while moving to the distal position has a lower impact. Nonetheless, the Bravos transit time correction algorithm yielded a lower average deviation from planned dose distribution for all plans evaluated, with 35 out of 36 plans having an average deviation lower than $1 \%$ while only 20 plans had an average deviation lower than $1 \%$ using the GammaMedplus iX algorithm.

If the same plans are delivered without transit time correction, the average dose deviation reaches a maximum of almost $20 \%$ for dwell times of 1.0 second and IDD of $1 \mathrm{~mm}$ while the maximum average dose deviation with transit time correction is $6 \%$ or less for the same plans. This shows that, regardless of the transit time correction algorithm used, the transit time correction is an essential feature for HDR BT treatments that must not be neglected. 

Future Perspectives 

Brachytherapy is a field of constant study, with approximately 1000 papers published per year during the last 5 years (2015-2019) containing the keyword brachytherapy (PubMed 01/01/2020). Nevertheless, in clinical practice, brachytherapy still lacks the technology applied for EBRT, leaving room for improvement in most stages of the treatment workflow. Consequently, brachytherapy is currently an operator dependent treatment technique where errors may be undetected. Some relevant issues mentioned in this thesis that should be subject of further investigation to improve brachytherapy treatments are:

\section{Afterloader QA and Applicator commissioning}

Both the afterloader QA and applicator commissioning currently rely on time integrated dwell position verification using radiochromic films as a standard practice. Such technique is not only cumbersome but also lacks the information regarding dwell times. Even the CamScale device implemented to the Bravos afterloading system, that relies on video cameras to verify dwell positions, could be better used if it also verified dwell times, unfortunately, the dwell time verification feature was not implemented to the system. Moreover, cameras with relatively high frame rate (240 fps) are already available in high-end smartphones. Using such cameras in devices like the CamScale could greatly expand its QA capabilities. Time resolved techniques (e.g. using IPs) should be adopted to evaluate, not only dwell positions but also dwell times and source trajectory inside opaque applicators. With technological progress and IP cost reduction, a paradigm change is expected for the next years, moving from time integrated to time resolved afterloader QA and applicator commissioning.

\section{Treatment planning}

Although TPSs from major companies have MBDCA options, the standard clinical practice still relies on TG43 calculations because MBDCA calculations are still relatively slow for dose optimization in clinical practice. However, with increase of computational power, improvement of MBDCA efficiency, and better tissue composition assignment (e.g. using dual energy CT images), it is expected to see MBDCA algorithms having a bigger role in treatment planning for the next years. This would also allow the development of more advanced applicator designs that can better shape the dose distribution according to the treatment requirements.

\section{In room imaging}

From an economical point of view, it may be more convenient to have the 
imaging system outside the brachytherapy room, so it can also be used for other treatment modalities. However, the main problem lies in the fact that the applicator positioning and/or patient geometry can change between the image acquisition and treatment delivery (e.g. bladder filling, gas pockets, patient movement). Suchowerska et al. [172] reported that imaging immediately before treatment delivery reduces maximum dose deviation from $67 \%$ to $9 \%$. Moreover, CBCT is already adopted as a standard inroom imaging technique for EBRT. Therefore, at least one imaging technique should be present in the brachytherapy treatment room to evaluate the changes that may have occurred and correct the treatment plan accordingly.

\section{Treatment delivery verification}

Currently, there are no treatment delivery verification systems routinely adopted for brachytherapy. The quality of the treatment relies on the assumption that both the afterloader and applicators were properly commissioned, that the patient geometry and applicator position hasn't changed between imaging and treatment, and on the operator skills. An error in any of these stages will result in an incorrect treatment delivery that will be unnoticed due to lack of verification. Efforts have been made towards in-vivo dosimetry, but more studies are necessary to find a cost-effective way for clinical implementation. For that reason, our research group recently received a substantial grant to work on this topic for the next 5 years.

\section{Transit dose}

The transit dose must not be neglected during a brachytherapy treatment. Although afterloaders already partially correct for the transit time and apply a correction to minimize its effect, it was demonstrated in this thesis that different transit time correction algorithms can result in different dose distributions even for afterloaders with a very similar source movement (when no transit time correction is applied). This is especially relevant for treatments using applicators with multiple channels or multiple needles (e.g. HDR prostate treatments), in which small dwell time variations combined yield big dose variations.

The transit dose component, however, is case specific, highly dependent on parameters like source speed, dwell times, IDDs, channels arrangement, transit time correction algorithm and region being evaluated. As an example, Fonseca et al. [122] simulated the transit dose component for 2 
prostate cases with the same prescribed dose of $10 \mathrm{~Gy}$; one with irradiation time of 200.9 seconds split in 113 dwell positions and other with irradiation time of 232.2 seconds split in 50 dwell positions. The average transit dose delivered to the prostate for the first case was $(11.1 \pm 0.5) \%$ of the prescribed dose while it was $(3.8 \pm 0.2) \%$ for the second case, both cases without transit time correction.

The transit dose component is unavoidable due to the limited source speed, but transit time correction algorithms should be improved in order to reduce dose distribution deviations. Ideally, the transit dose should be accounted by the TPS during the planning optimization stage, including its effects to clinical parameters.

With an increase in computational power and MBDCA efficiency, an improvement in patient specific dose optimization is expected within the next years, taking in consideration tissue and applicator composition. Such advances would allow more complex treatments and applicator models, which are expected to be followed by ways to ensure that the planned treatment is properly delivered, including more precise and accurate QA and commissioning methods, and real time treatment verification. 

A

Appendix A - Nonlinearity Correction 

The response of the panel is linear with the air kerma strength (Sk) for the whole panel for most of the cases as observed irradiating the panel with different Sk values. However, a nonlinear behavior was observed for one region of the panel which was clearly noticeable for large distances between the source and the panel.

Figure A.1a shows profiles obtained for both Sk (Sk(high) and Sk(Low)*1.67) with the source at $7 \mathrm{~cm}$ (left scale) and $20 \mathrm{~cm}$ (right scale) from the panel. The distance between the source and the panel (z) was the same for both Sk(high) and Sk(Low) whilst the xy position was shifted of around $1 \mathrm{~cm}$. The positioning difference (xy) should not affect the response in the region of high intensities close to the center of the phantom. Therefore, the registered results (Sk(high) and Sk(Low)*1.67) should be almost the same considering the experimental uncertainties, except for differences, due to back scattering conditions, in the low dose region close to the boundaries of the phantom.
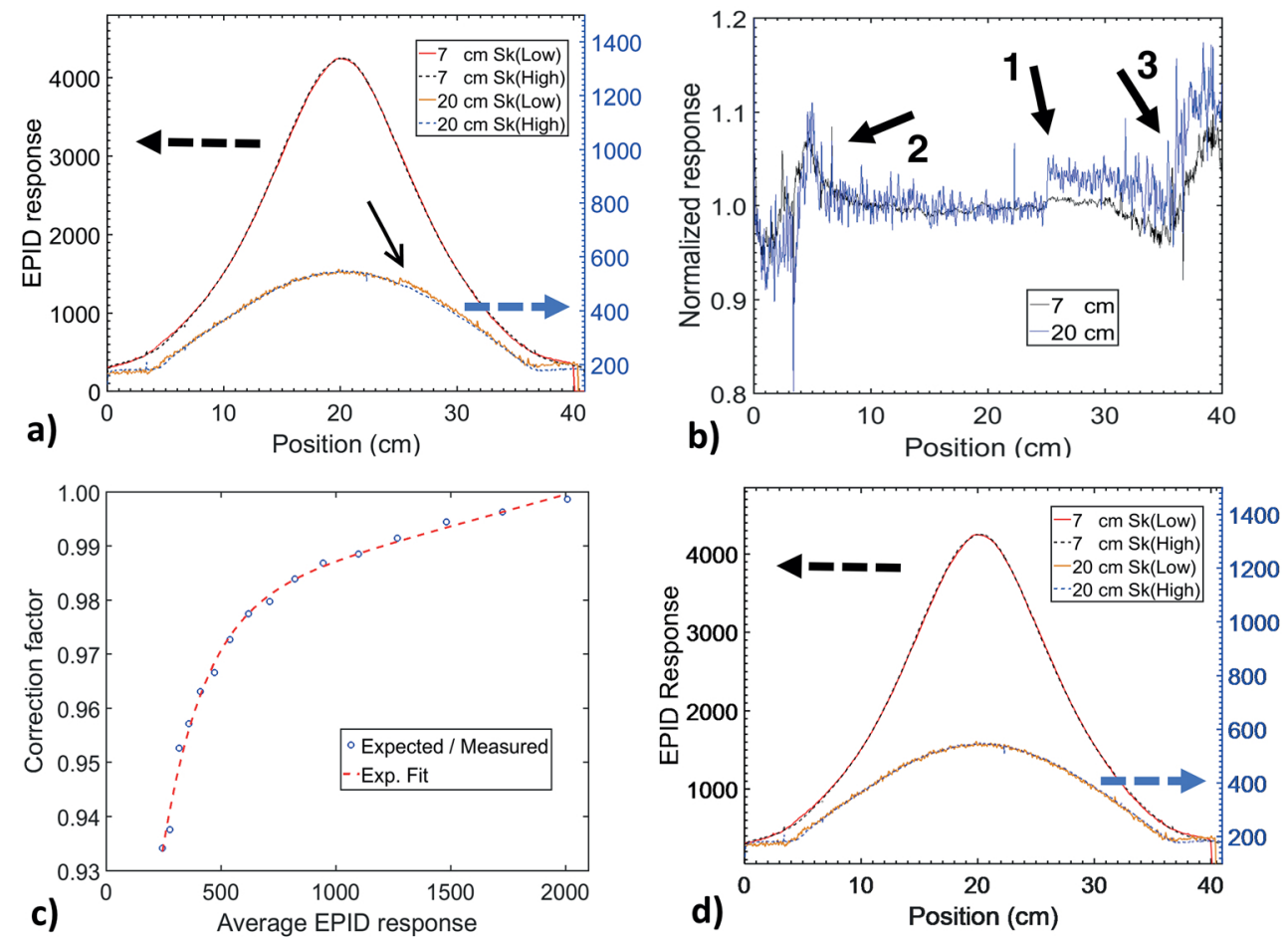

Figure A.1. a) response profile for two different Sk (Sk(Low) was multiplied by 1.67) with the source, within a water phantom, at $7 \mathrm{~cm}$ (left scale) and $20 \mathrm{~cm}$ (right scale) from the panel. The black arrow shows the region where an abrupt shift was observed. b) ratio between the profiles shown in panel a. c) correction factor as a function of the mean IP response at the boundaries of the response shift. d) corrected profiles $(7 \mathrm{~cm}$ left scale and $20 \mathrm{~cm}$ right scale).

When corrected by the Sk the profiles show a good agreement with no differences 
noticeable for high intensities. However, one region of the panel (Figure A.la - black arrow) shows an overresponse that increases with the distance. Figure A.1b shows the ratio between the profiles shown in Figure A.1a. Results are noisy for the $20 \mathrm{~cm}$ distance, but a clear threshold (Figure A.1b arrow 1) is visible around $25 \mathrm{~cm}$ which coincides with the boundary of a subpanel of the detector. The large variation close to the extremes of the field due to differences in the scattering conditions are indicated in Figure A.1b (arrows 2 and 3). The profiles shown in Figure A.1b have a smooth behavior with differences increasing towards the edges, except for an abrupt response shift at 25.

The response shift was corrected by multiplying the response of all the pixels after the position $25 \mathrm{~cm}$ by a correction factor (Figure A.1c) that follows the behavior a two exponential curves. The correction factor corresponds to the ratio between the IP response immediately before and after the abrupt shift in Figure A.1b (arrow 1) and was measured for a wide IP response range. Figure A.1d shows the corrected response. The response shift happens in the y direction which is used to obtain the $\mathrm{z}$ coordinate of the source making this correction especially relevant with $\mathrm{z}$ differences up to $2 \mathrm{~cm}$ (source at $22 \mathrm{~cm}$ from the panel and Sk(low)) for very low response values. 
B

Appendix B - Dwell Position Verification 

The dwell positions measured using the IP were verified comparing the results against EBT3 radiochromic film (GAFCHROMIC, Ashland Specialty Ingredients, Bridgewater, NJ) measurements. Figure B.1a shows an EBT3 film placed on top of the IP $(\mathrm{a} 1 \mathrm{~cm}$ thick PMMA plate was added between the film and the panel to avoid IP response saturation). Two brachytherapy needles and two catheters (more flexible allowing different curvatures) were placed right on top of the film and an irradiation was performed with 55 dwell positions, $1 \mathrm{~cm}$ inter dwell distance and 3.3 seconds dwell time for each dwell position (Sk = $24420 \mathrm{U}$ ). IP images were acquired during the same irradiation to eliminate the afterloader positioning uncertainty between different measurements. The IP dwell positions were calculated using the Gaussian fit described in the manuscript. The film was processed using a similar fit applied the local maxima.

a)
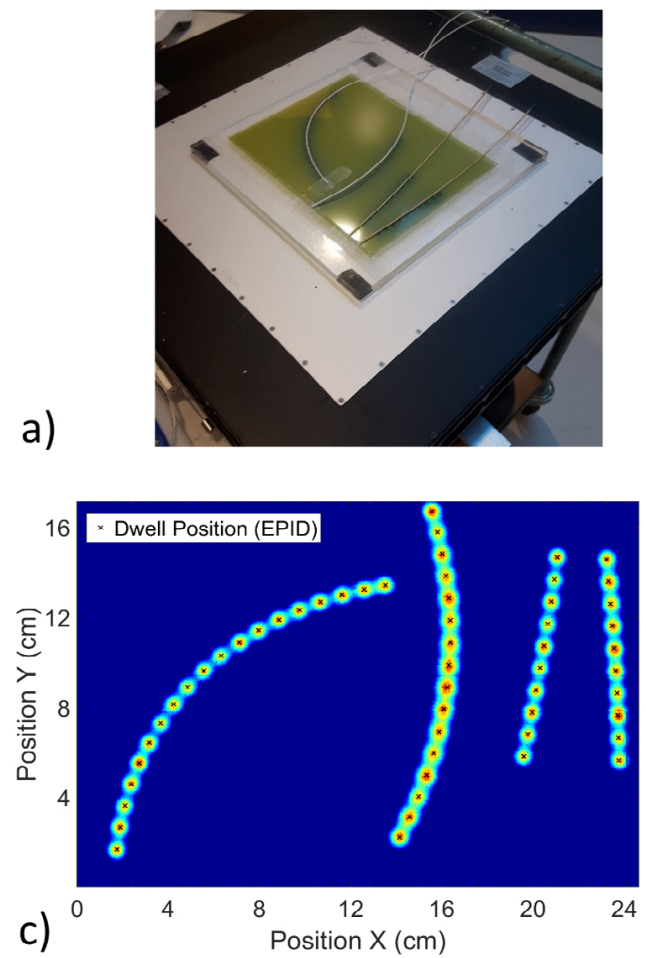
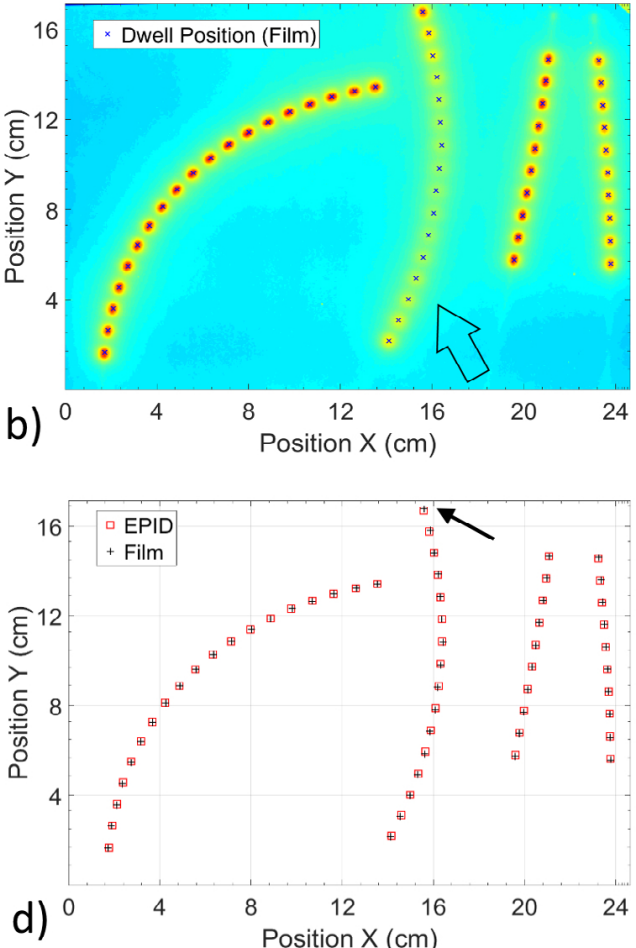

Figure B.1. (a) Experimental setup used to compare film and IP measurements. (b) EBT3 film response; (c) integrated IP response (only for visualization purposes). (d) Comparison between the dwell position. The arrow indicates the dwell position with the largest difference.

Figure B.1b shows the film response and the location of the identified dwell positions. Different intensities can be observed due to small air gaps between the needles/catheters and the films since only the extremities were fixed. Intensity differences are more significant for the second catheter (Figure B.1bblack arrow), whose center was few 
millimeters above the surface of the film. These differences are not relevant since they affect equally both film and IP measurements. Figure B.1c shows the integrated IP response and the identified dwell positions. The dwell positions were measured frame by frame as described in the manuscript. However, the integrated image was processed for visualization purposes. Each IP frame was normalized to its maximum (so no intensity differences can be observed in Figure B.1c and raised to the power of 20 (arbitrary number) to increase the difference between the dwell position and its surroundings so the dwell positions are clearly visible in the integrated image. It was necessary since the image becomes blurrier with distance and the IP was at least $1 \mathrm{~cm}$ below the source.

Figure B.1d shows the dwell positions measured using both film and IP. The mean difference is approximately $0 \mathrm{~cm}(<1 E 5)$ with a standard deviation of $0.03 \mathrm{~cm}$ (Type Al $\sigma$ ) and a maximum difference of $0.1 \mathrm{~cm}$. The mean difference is close to zero since the images were registered to account for the different alignment while scanning the film. The image registration algorithm minimizes the differences leading to a very low mean difference. Therefore, the mean difference is not very representative whilst the standard deviation should be considered for a more reliable comparison. The maximum difference occurs close to the edge of the film (Figure B.1d arrow) where the fit may underperform since less points are available.

The IP measurement uncertainty estimated in this work $(<0.02 \mathrm{~cm}$ without repositioning $)$ is very consistent with the differences observed, which includes the uncertainties of both methods in addition to the image registration. 
Appendix C - Monte Carlo Simulations Parameters And Uncertainty Analysis 



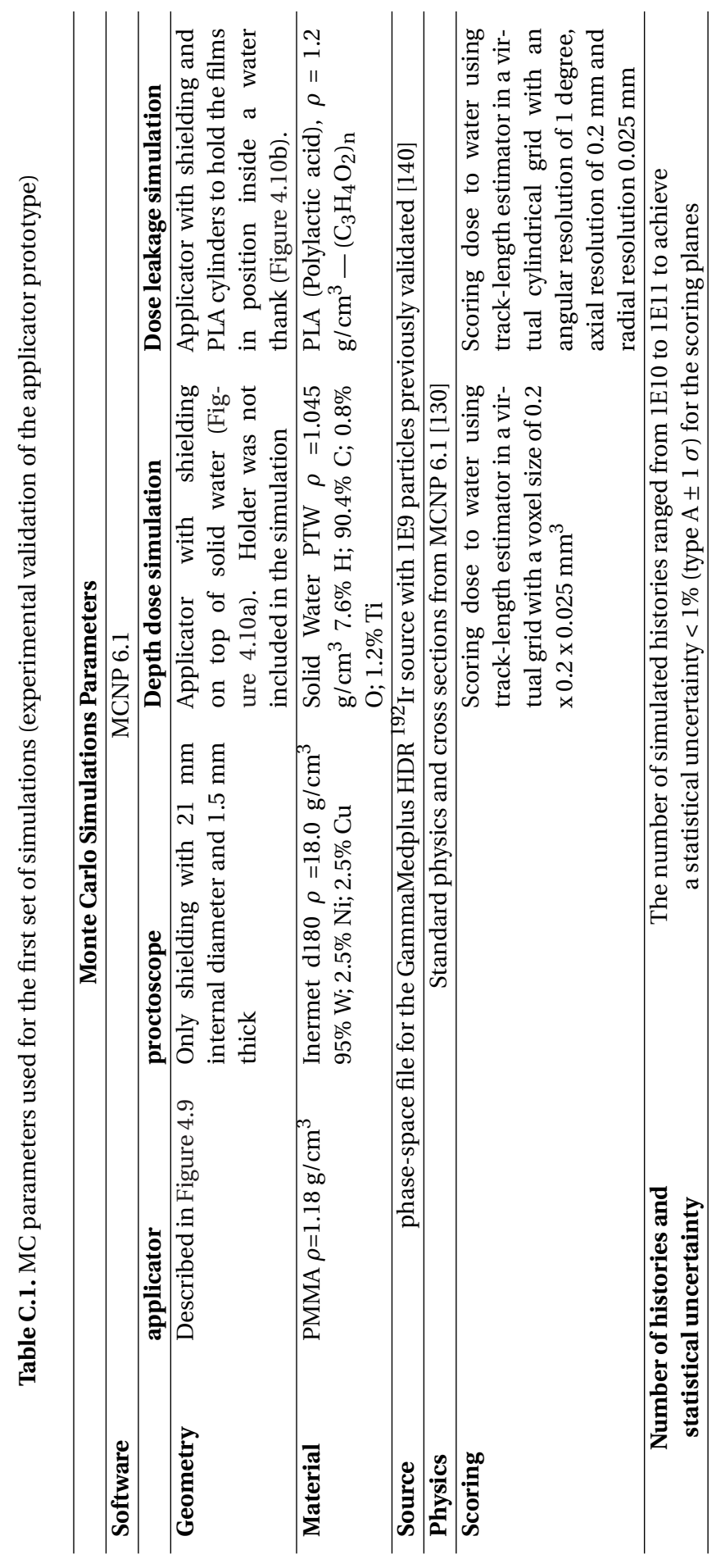




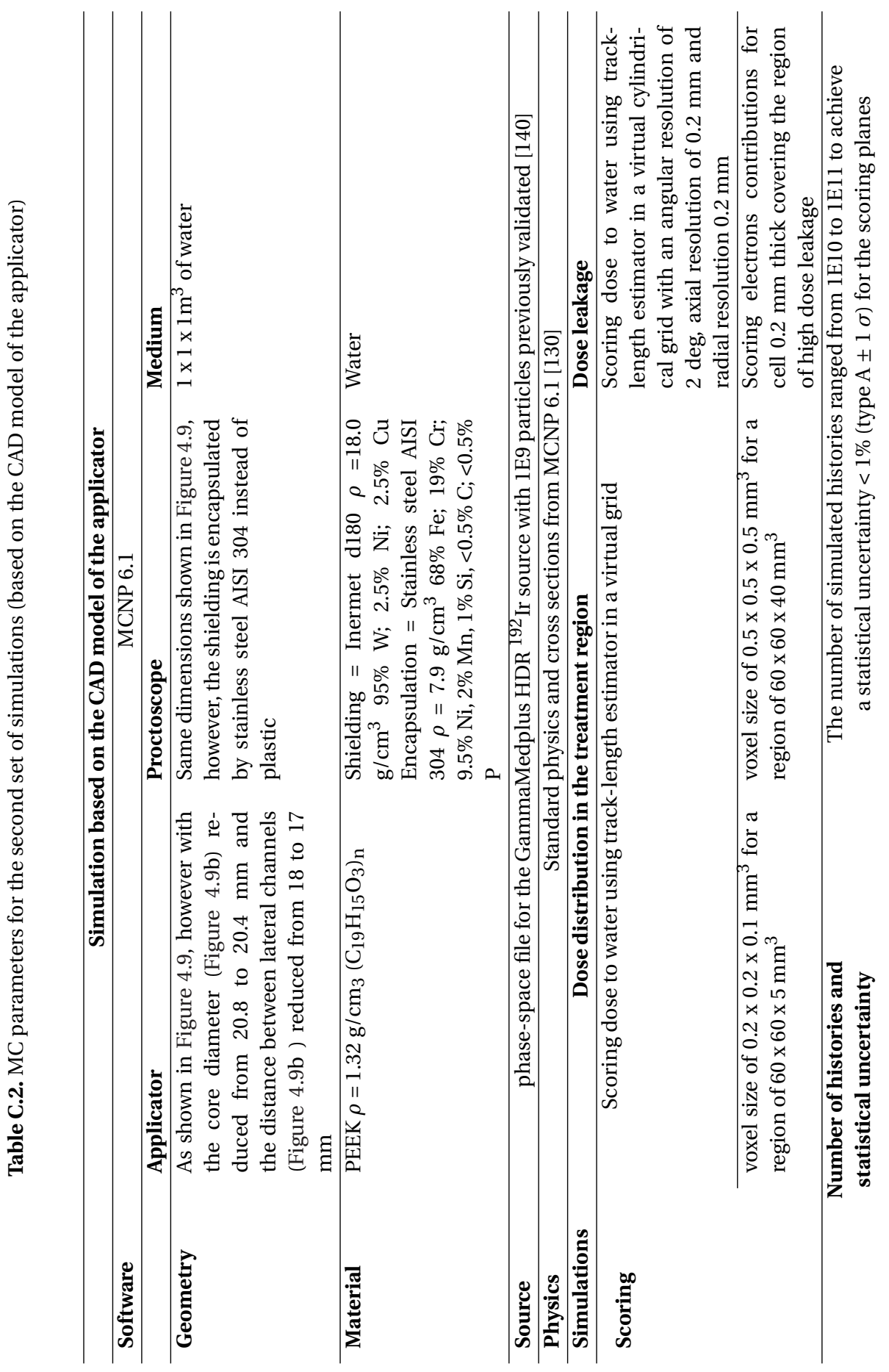




\section{UNCERTAINTY ANALYSIS}

The uncertainty analysis for the experimental validation was performed based on Table XII of the TG43 update [19]. Results are summarized in Table 5.1 showing $1 \sigma$.

Table C.3. Uncertainty analysis for the experiments of depth dose measurements and dose leakage measured with EBT-XD films. All uncertainties are expressed by $1 \sigma$.

Depth dose measurements

\begin{tabular}{|c|c|c|c|c|c|c|}
\hline Scoring depth & \multicolumn{2}{|c|}{$1 \mathrm{~mm}$} & \multicolumn{2}{|c|}{$2 \mathrm{~mm}$} & \multicolumn{2}{|c|}{$5 \mathrm{~mm}$} \\
\hline Component & Tyре A & Type B & Type A & Type B & TypeA & Type B \\
\hline Repetitive measurements & $1.7 \%$ & & $1.3 \%$ & & $0.8 \%$ & \\
\hline Film calibration and readout & & $2.0 \%$ & & $2.0 \%$ & & $2.0 \%$ \\
\hline Scoring distance & & $6.8 \%$ & & $5.2 \%$ & & $3.1 \%$ \\
\hline Quadrature Sum & $1.7 \%$ & $7.1 \%$ & $1.3 \%$ & $5.6 \%$ & $0.8 \%$ & $3.7 \%$ \\
\hline Total uncertainty & \multicolumn{2}{|c|}{$7.3 \%$} & \multicolumn{2}{|c|}{$5.8 \%$} & \multicolumn{2}{|c|}{$3.8 \%$} \\
\hline
\end{tabular}

Dose leakage measurements

\begin{tabular}{lcccc}
\hline Scoring distance & \multicolumn{2}{c}{$\mathbf{1 ~ \mathbf { ~ m }}$} & \multicolumn{2}{c}{$\mathbf{2 ~ \mathbf { ~ m }}$} \\
\hline Component & Type A & Type B & Type A & Type B \\
Repetitive measurements & $1.0 \%$ & & $1.0 \%$ & \\
Film calibration and readout & \multicolumn{2}{c}{$2.0 \%$} & & $2.0 \%$ \\
Scoring distance* & \multicolumn{2}{c}{$1.7 \%$} & $1.3 \%$ \\
Quadrature Sum & $1.0 \%$ & $2.6 \%$ & $1.0 \%$ & $2.4 \%$ \\
Total uncertainty & \multicolumn{2}{c}{$2.8 \%$} & $2.6 \%$ \\
\hline *The effect that the uncertainty on the scoring distance would have on the dose delivered to the film \\
was simulated with Monte Carlo
\end{tabular}

\section{EBT-XD calibration and readout}

The EBT-XD user guide [145] reports an uncertainty of $2 \%$ when using the triple channel method. Therefore, the uncertainty for the film calibration and readout combined for this study was estimated to be $2.0 \%$.

\section{Films irradiation during experiments}

The largest uncertainty regarding the dose delivered to the films during the experiments is the distance between the source and the films due to the steep dose gradient close to the source. The HDR ${ }^{192}$ Ir source has a diameter of $0.9 \mathrm{~mm}$ while the lumen of the catheter is $1.2 \mathrm{~mm}$, resulting in an uncertainty of $\pm 0.15 \mathrm{~mm}$ on the distance between the source and the contact surface of the applicator. The uncertainty on the distance between the contact surface of the applicator and the radiochromic film is estimated to be $\pm 0.2 \mathrm{~mm}$, resulting in an uncertainty between the center of source and scoring depth position of \pm $0.35 \mathrm{~mm}$, which would have an impact on the dose distribution of $6.8 \%, 5.2 \%$ and $3.1 \%$ ( 1 $\sigma$ ) at scoring depth of $1 \mathrm{~mm}, 2 \mathrm{~mm}$ and $5 \mathrm{~mm}$, respectively, using the inverse-square law approximation. 
For the dose leakage experiments, the uncertainty on the film position is estimated at $\pm 0.25 \mathrm{~mm}$, which implies a dose leakage uncertainty for the points with highest dose leakage of $1.7 \%$ and $1.3 \%(1 \sigma)$ at scoring distances of $1 \mathrm{~mm}$ and $2 \mathrm{~mm}$, respectively, according to the MC simulations. 
References 

1. Williamson, J.F., Brachytherapy technology and physics practice since 1950: a half-century of progress. Phys Med Biol, 2006. 51(13): p. R303-25.

2. Mazeron, R., J. Champoudry, J. Gilmore, I. Dumas, J. Goulart, A.S. Oberlander, E. Rivin del Campo, I. Diallo, D. Lefkopoulos, and C. Haie-Meder, Intrafractional organs movement in three-dimensional image-guided adaptive pulsed-dose-rate cervical cancer brachytherapy: assessment and dosimetric impact. Brachytherapy, 2015. 14(2): p. 260-6.

3. Simha, V., F.D. Patel, S.C. Sharma, B. Rai, A.S. Oinam, R. krishnatry, and B. Dhanireddy, Evaluation of intrafraction motion of the organs at risk in image-based brachytherapy of cervical cancer. Brachytherapy, 2014. 13(6): p. 562-7.

4. Georg, D., J. Hopfgartner, J. Gora, P. Kuess, G. Kragl, D. Berger, N. Hegazy, G. Goldner, and P. Georg, Dosimetric considerations to determine the optimal technique for localized prostate cancer among external photon, proton, or carbon-ion therapy and high-dose-rate or low-dose-rate brachytherapy. Int J Radiat Oncol Biol Phys, 2014. 88(3): p. 715-22.

5. Gill, B.S., J.F. Lin, T.C. Krivak, P. Sukumvanich, R.A. Laskey, M.S. Ross, J.L. Lesnock, and S. Beriwal, National Cancer Data Base analysis of radiation therapy consolidation modality for cervical cancer: the impact of new technological advancements. Int J Radiat Oncol Biol Phys, 2014. 90(5): p. 1083-90.

6. Tanderup, K., P.J. Eifel, C.M. Yashar, R. Potter, and P.W. Grigsby, Curative radiation therapy for locally advanced cervical cancer: brachytherapy is NOT optional. Int J Radiat Oncol Biol Phys, 2014. 88(3): p. 537-9.

7. Han, K., M. Milosevic, A. Fyles, M. Pintilie, and A.N. Viswanathan, Trends in the utilization of brachytherapy in cervical cancer in the United States. Int J Radiat Oncol Biol Phys, 2013. 87(1): p. 111-9.

8. Shah, C., T.B. Lanni, Jr., M.I. Ghilezan, G.S. Gustafson, K.S. Marvin, H. Ye, F.A. Vicini, and A.A. Martinez, Brachytherapy provides comparable outcomes and improved cost-effectiveness in the treatment of low/intermediate prostate cancer. Brachytherapy, 2012. 11(6): p. 441-5.

9. Song, W.Y., K. Tanderup, and B. Pieters, Is Brachytherapy Cost Effective? peter orio, BenJamin durKee, thomaS lanni, Yolande lievenS, and daniel petereit, in Emerging Technologies in Brachytherapy. 2017, CRC Press. p. 397-404.

10. Petereit, D.G., S.J. Frank, A.N. Viswanathan, B. Erickson, P. Eifel, P.L. Nguyen, and D.E. Wazer, Brachytherapy: where has it gone? J Clin Oncol, 2015. 33(9): p. 980-2.

11. Orio, P.F, 3rd, P.L. Nguyen, I. Buzurovic, D.W. Cail, and Y.W. Chen, The decreased use of brachytherapy boost for intermediate and high-risk prostate cancer despite evidence supporting its effectiveness. Brachytherapy, 2016. 15(6): p. 701-706.

12. Mahmood, U., T. Pugh, S. Frank, L. Levy, G. Walker, W. Haque, M. Koshy, W. Graber, D. Swanson, K. Hoffman, D. Kuban, and A. Lee, Declining use of brachytherapy for the treatment of prostate cancer. Brachytherapy, 2014. 13(2): p. 157-62.

13. Rajagopalan, M.S., K.M. Xu, J. Lin, K. Hansen, P. Sukumvanich, T.C. Krivak, J.L. Kelley, and S. Beriwal, Patterns of care and brachytherapy boost utilization for vaginal cancer in the United States. Pract Radiat Oncol, 2015. 5(1): p. 56-61.

14. Song, W.Y., K. Tanderup, and B. Pieters, Emerging technologies in brachytherapy. 2017, CRC Press, Taylor \& Francis Group: Boca Raton, FL. 
15. Buzurovic, I.M., J.L. Hansen, M.S. Bhagwat, D.A. O’Farrell, S. Friesen, T.C. Harris, A.L. Damato, R.A. Cormack, N.E. Martin, and P.M. Devlin, Clinical implementation of a novel applicator in high-dose-rate brachytherapy treatment of esophageal cancer. J Contemp Brachytherapy, 2016. 8(4): p. 319-25.

16. Granero, D., C. Candela-Juan, F. Ballester, Z. Ouhib, J. Vijande, J. Richart, and J. Perez-Calatayud, Commissioning and quality assurance procedures for the HDR Valencia skin applicators. J Contemp Brachytherapy, 2016. 8(5): p. 441-447.

17. Fonseca, G.P., M.R. Van den Bosch, R. Voncken, M. Podesta, and F. Verhaegen, A novel system for commissioning brachytherapy applicators: example of a ring applicator. Phys Med Biol, 2017. 62(21): p. $8360-8375$.

18. Whitaker, M., G. Hruby, A. Lovett, and N. Patanjali, Prostate HDR brachytherapy catheter displacement between planning and treatment delivery. Radiother Oncol, 2011. 101(3): p. 490-4.

19. Rivard, M.J., B.M. Coursey, L.A. DeWerd, W.F. Hanson, M.S. Huq, G.S. Ibbott, M.G. Mitch, R. Nath, and J.F. Williamson, Update of AAPM Task Group No. 43 Report: A revised AAPM protocol for brachytherapy dose calculations. Med Phys, 2004. 31(3): p. 633-74.

20. Rivard, M.J., W.M. Butler, L.A. DeWerd, M.S. Huq, G.S. Ibbott, Z. Li, M.G. Mitch, R. Nath, and J.F. Williamson, Erratum: Update of AAPM Task Group No. 43 Report: A revised AAPM protocol for brachytherapy dose calculations [Med. Phys. 31, 633-674 (2004)]. Medical Physics, 2004. 31(12): p. 3532-3533.

21. Rivard, M.J., W.M. Butler, L.A. Dewerd, M.S. Huq, G.S. Ibbott, A.S. Meigooni, C.S. Melhus, M.G. Mitch, R. Nath, and J.F. Williamson, Supplement to the 2004 update of the AAPM Task Group No. 43 Report. Medical physics., 2007. 34(6): p. 2187.

22. Valentin, J. and P. International Commission on Radiation, Prevention of high-dose-rate brachytherapy accidents. ICRP Publication 97. Ann ICRP, 2005. 35(2): p. 1-51.

23. Honderden vrouwen onjuist bestraald in Maastricht. 2014 [cited 2019 01/11/2019]; Available from: https://www.rtlnieuws.nl/node/1807366.

24. McQuigge, M. 25 women may have received faulty cancer treatment at Hamilton hospital. 2019 [cited 2019 01/11/2019]; Available from: https://globalnews.ca/news/4811987/faulty-cancer-treatmenthamilton-hospital/.

25. Bellezzo, M., J.A. Baeza, R. Voncken, B. Reniers, F. Verhaegen, and G.P. Fonseca, Mechanical evaluation of the Bravos afterloader system for HDR brachytherapy. Brachytherapy, 2019. 18(6): p. 852-862.

26. Varian Medical Systems, P.A., CA, Bravos Reference Guide, Version 1.0. 2018.

27. Wong, T.P., W. Fernando, P.N. Johnston, and I.F. Bubb, Transit dose of an Ir-192 high dose rate brachytherapy stepping source. Phys Med Biol, 2001. 46(2): p. 323-31.

28. Sahoo, N., Measurement of transit time of a remote after-loading high dose rate brachytherapy source. Med Phys, 2001. 28(8): p. 1786-90.

29. Palmer, A. and B. Mzenda, Performance assessment of the BEBIG MultiSource high dose rate brachytherapy treatment unit. Phys Med Biol, 2009. 54(24): p. 7417-34. 
30. Fonseca, G.P., R.S. Viana, M. Podesta, R.A. Rubo, C.P. de Sales, B. Reniers, H. Yoriyaz, and F. Verhaegen, HDR 192Ir source speed measurements using a high speed video camera. Med Phys, 2015. 42(1): p. 412-5.

31. Hoskin, P.J., A. Colombo, A. Henry, P. Niehoff, T. Paulsen Hellebust, F.A. Siebert, and G. Kovacs, GEC/ESTRO recommendations on high dose rate afterloading brachytherapy for localised prostate cancer: an update. Radiother Oncol, 2013. 107(3): p. 325-32.

32. Holm, H.H. and J. Gammelgaard, Ultrasonically guided precise needle placement in the prostate and the seminal vesicles. J Urol, 1981. 125(3): p. 385-7.

33. Holm, H.H., N. Juul, J.F. Pedersen, H. Hansen, and I. Strøyer, Transperineal 125iodine seed implantation in prostatic cancer guided by transrectal ultrasonography. The Journal of urology, 1983. 130(2): p. 283-6.

34. Sharma, D.N., G.K. Rath, S. Thulkar, S. Kumar, V. Subramani, and P.K. Julka, Use of transrectal ultrasound for high dose rate interstitial brachytherapy for patients of carcinoma of uterine cervix. J Gynecol Oncol, 2010. 21(1): p. 12-7.

35. Schmid, M.P., N. Nesvacil, R. Potter, G. Kronreif, and C. Kirisits, Transrectal ultrasound for image-guided adaptive brachytherapy in cervix cancer - An alternative to MRI for target definition? Radiother Oncol, 2016. 120(3): p. 467-472.

36. Nesvacil, N., M.P. Schmid, R. Potter, G. Kronreif, and C. Kirisits, Combining transrectal ultrasound and CT for image-guided adaptive brachytherapy of cervical cancer: Proof of concept. Brachytherapy, 2016. 15(6): p. 839-844.

37. Reniers, B. and F. Verhaegen, Technical note: cone beam CT imaging for 3D image guided brachytherapy for gynecological HDR brachytherapy. Med Phys, 2011. 38(5): p. 2762-7.

38. Orcutt, K.P., B. Libby, L.L. Handsfield, G. Moyer, and T.N. Showalter, CT-on-rails-guided HDR brachytherapy: single-room, rapid-workflow treatment delivery with integrated image guidance. Future Oncol, 2014. 10(4): p. 569-75.

39. Jaffray, D.A., M.C. Carlone, M.F. Milosevic, S.L. Breen, T. Stanescu, A. Rink, H. Alasti, A. Simeonov, M.C. Sweitzer, and J.D. Winter, A facility for magnetic resonance-guided radiation therapy. Semin Radiat Oncol, 2014. 24(3): p. 193-5.

40. Beaulieu, L., A. Carlsson Tedgren, J.F. Carrier, S.D. Davis, F. Mourtada, M.J. Rivard, R.M. Thomson, F. Verhaegen, T.A. Wareing, and J.F. Williamson, Report of the Task Group 186 on model-based dose calculation methods in brachytherapy beyond the TG-43 formalism: current status and recommendations for clinical implementation. Med Phys, 2012. 39(10): p. 6208-36.

41. Ahnesjo, A., Collapsed cone convolution of radiant energy for photon dose calculation in heterogeneous media. Med Phys, 1989. 16(4): p. 577-92.

42. Williams, M.M.R., Computational methods of neutron transport. Annals of Nuclear Energy, 1985. 12(2): p. 101.

43. Rogers, D.W., Fifty years of Monte Carlo simulations for medical physics. Phys Med Biol, 2006. 51(13): p. R287-301. 
44. Landry, G., M.J. Rivard, J.F. Williamson, and F. Verhaegen, Monte Carlo Methods and Applications for Brachytherapy Dosimetry and Treatment Planning. Monte Carlo Techniques in Radiation Therapy, 2013: p. 125-143.

45. Fonseca, G.P., B. Reniers, G. Landry, S. White, M. Bellezzo, P.C. Antunes, C.P. de Sales, E. Welteman, H. Yoriyaz, and F. Verhaegen, A medical image-based graphical platform - features, applications and relevance for brachytherapy. Brachytherapy, 2014. 13(6): p. 632-9.

46. Petrokokkinos, L., K. Zourari, E. Pantelis, A. Moutsatsos, P. Karaiskos, L. Sakelliou, I. Seimenis, E. Georgiou, and P. Papagiannis, Dosimetric accuracy of a deterministic radiation transport based 192Ir brachytherapy treatment planning system. Part II: Monte Carlo and experimental verification of a multiple source dwell position plan employing a shielded applicator. Med Phys, 2011. 38(4): p. 198192.

47. Ma, Y., J. Vijande, F. Ballester, A.C. Tedgren, D. Granero, A. Haworth, F. Mourtada, G.P. Fonseca, K. Zourari, P. Papagiannis, M.J. Rivard, F.A. Siebert, R.S. Sloboda, R. Smith, M.J.P. Chamberland, R.M. Thomson, F. Verhaegen, and L. Beaulieu, A generic TG-186 shielded applicator for commissioning model-based dose calculation algorithms for high-dose-rate (192) Ir brachytherapy. Med Phys, 2017. 44(11): p. 5961-5976.

48. Fonseca, G.P., M. Podesta, M. Bellezzo, M.R. Van den Bosch, L. Lutgens, B.G.L. Vanneste, R. Voncken, E.J. Van Limbergen, B. Reniers, and F. Verhaegen, Online pretreatment verification of high-dose rate brachytherapy using an imaging panel. Physics in Medicine \& Biology, 2017. 62(13): p. 5440-5461.

49. Smith, R.L., M.L. Taylor, L.N. McDermott, A. Haworth, J.L. Millar, and R.D. Franich, Source position verification and dosimetry in HDR brachytherapy using an EPID. Med Phys, 2013. 40(11): p. 111706.

50. Verhaegen, F, S. Palefsky, D. Rempel, and E. Poon, Imaging with Iridium photons: an application in brachytherapy. Proceedings of SPIE, 2007. 6510: p. 65103S-11.

51. Papagiannis, P., E. Pantelis, and P. Karaiskos, Current state of the art brachytherapy treatment planning dosimetry algorithms. Br J Radiol, 2014. 87(1041): p. 20140163.

52. Kertzscher, G., A. Rosenfeld, S. Beddar, K. Tanderup, and J.E. Cygler, In vivo dosimetry: trends and prospects for brachytherapy. Br J Radiol, 2014. 87(1041): p. 20140206.

53. Tanderup, K., S. Beddar, C.E. Andersen, G. Kertzscher, and J.E. Cygler, In vivo dosimetry in brachytherapy. Medical physics, 2013. 40(7): p. 070902.

54. Reniers, B., G. Landry, R. Eichner, A. Hallil, and F. Verhaegen, In vivo dosimetry for gynaecological brachytherapy using a novel position sensitive radiation detector: feasibility study. Med Phys, 2012. 39(4): p. 1925-35.

55. Andersen, C.E., S.K. Nielsen, J.C. Lindegaard, and K. Tanderup, Time-resolved in vivo luminescence dosimetry for online error detection in pulsed dose-rate brachytherapy. Med Phys, 2009. 36(11): p. 503343.

56. Smith, R.L., A. Haworth, V. Panettieri, J.L. Millar, and R.D. Franich, A method for verification of treatment delivery in HDR prostate brachytherapy using a flat panel detector for both imaging and source tracking. Medical Physics, 2016. 43(5): p. 2435-2442.

57. Smith, R.L., A. Haworth, V. Panettieri, J.L. Millar, and R.D. Franich, 3D catheter reconstruction in HDR prostate brachytherapy for pre-treatment verification using a flat panel detector. Physica Medica, 2017. 39: p. 121-131. 
58. Nath, R., L.L. Anderson, J.A. Meli, A.J. Olch, J.A. Stitt, and J.F. Williamson, Code of practice for brachytherapy physics: Report of the AAPM Radiation Therapy Committee Task Group No. 56. Medical Physics, 1997. 24(10): p. 1557-1598.

59. Global Burden of Disease Cancer, C., C. Fitzmaurice, C. Allen, R.M. Barber, L. Barregard, Z.A. Bhutta, H. Brenner, D.J. Dicker, O. Chimed-Orchir, R. Dandona, L. Dandona, T. Fleming, M.H. Forouzanfar, J. Hancock, R.J. Hay, R. Hunter-Merrill, C. Huynh, H.D. Hosgood, C.O. Johnson, J.B. Jonas, J. Khubchandani, G.A. Kumar, M. Kutz, Q. Lan, H.J. Larson, X. Liang, S.S. Lim, A.D. Lopez, M.F. MacIntyre, L. Marczak, N. Marquez, A.H. Mokdad, C. Pinho, F. Pourmalek, J.A. Salomon, J.R. Sanabria, L. Sandar, B. Sartorius, S.M. Schwartz, K.A. Shackelford, K. Shibuya, J. Stanaway, C. Steiner, J. Sun, K. Takahashi, S.E. Vollset, T. Vos, J.A. Wagner, H. Wang, R. Westerman, H. Zeeb, L. Zoeckler, F. Abd-Allah, M.B. Ahmed, S. Alabed, N.K. Alam, S.F. Aldhahri, G. Alem, M.A. Alemayohu, R. Ali, R. Al-Raddadi, A. Amare, Y. Amoako, A. Artaman, H. Asayesh, N. Atnafu, A. Awasthi, H.B. Saleem, A. Barac, N. Bedi, I. Bensenor, A. Berhane, E. Bernabe, B. Betsu, A. Binagwaho, D. Boneya, I. Campos-Nonato, C. Castaneda-Orjuela, F. Catala-Lopez, P. Chiang, C. Chibueze, A. Chitheer, J.Y. Choi, B. Cowie, S. Damtew, J. das Neves, S. Dey, S. Dharmaratne, P. Dhillon, E. Ding, T. Driscoll, D. Ekwueme, A.Y. Endries, M. Farvid, F. Farzadfar, J. Fernandes, F. Fischer, G.H. TT, A. Gebru, S. Gopalani, A. Hailu, M. Horino, N. Horita, A. Husseini, I. Huybrechts, M. Inoue, F. Islami, M. Jakovljevic, S. James, M. Javanbakht, S.H. Jee, A. Kasaeian, M.S. Kedir, Y.S. Khader, Y.H. Khang, D. Kim, J. Leigh, S. Linn, R. Lunevicius, H.M.A. El Razek, R. Malekzadeh, D.C. Malta, W. Marcenes, D. Markos, Y.A. Melaku, K.G. Meles, W. Mendoza, D.T. Mengiste, T.J. Meretoja, T.R. Miller, K.A. Mohammad, A. Mohammadi, S. Mohammed, M. Moradi-Lakeh, G. Nagel, D. Nand, Q. Le Nguyen, S. Nolte, F.A. Ogbo, K.E. Oladimeji, E. Oren, M. Pa, E.K. Park, D.M. Pereira, D. Plass, M. Qorbani, A. Radfar, A. Rafay, M. Rahman, S.M. Rana, K. Soreide, M. Satpathy, M. Sawhney, S.G. Sepanlou, M.A. Shaikh, J. She, I. Shiue, H.R. Shore, M.G. Shrime, S. So, S. Soneji, V. Stathopoulou, K. Stroumpoulis, M.B. Sufiyan, B.L. Sykes, R. TabaresSeisdedos, F. Tadese, B.A. Tedla, G.A. Tessema, J.S. Thakur, B.X. Tran, K.N. Ukwaja, B.S.C. Uzochukwu, V.V. Vlassov, E. Weiderpass, M. Wubshet Terefe, H.G. Yebyo, H.H. Yimam, N. Yonemoto, M.Z. Younis, C. Yu, Z. Zaidi, M.E.S. Zaki, Z.M. Zenebe, C.J.L. Murray and M. Naghavi, Global, Regional, and National Cancer Incidence, Mortality, Years of Life Lost, Years Lived With Disability, and Disability-Adjusted Life-years for 32 Cancer Groups, 1990 to 2015: A Systematic Analysis for the Global Burden of Disease Study. JAMA Oncol, 2017. 3(4): p. 524-548.

60. Maag Darm Lever Stichting 2015 Available from: https://www.mlds.nl/kanker/endeldarmkanker/.

61. Konanz, J., F. Herrle, C. Weiss, S. Post, and P. Kienle, Quality of life of patients after low anterior, intersphincteric, and abdominoperineal resection for rectal cancer-a matched-pair analysis. Int J Colorectal Dis, 2013. 28(5): p. 679-88.

62. Fazio, V.W., M. Zutshi, F.H. Remzi, Y. Parc, R. Ruppert, A. Furst, J. Celebrezze, Jr., S. Galanduik, G. Orangio, N. Hyman, L. Bokey, E. Tiret, B. Kirchdorfer, D. Medich, M. Tietze, T. Hull, and J. Hammel, A randomized multicenter trial to compare long-term functional outcome, quality of life, and complications of surgical procedures for low rectal cancers. Ann Surg, 2007. 246(3): p. 481-8; discussion 488-90.

63. Maas, M., P.J. Nelemans, V. Valentini, P. Das, C. Rodel, L.J. Kuo, F.A. Calvo, J. Garcia-Aguilar, R. GlynneJones, K. Haustermans, M. Mohiuddin, S. Pucciarelli, W. Small, Jr., J. Suarez, G. Theodoropoulos, S. Biondo, R.G. Beets-Tan, and G.L. Beets, Long-term outcome in patients with a pathological complete response after chemoradiation for rectal cancer: a pooled analysis of individual patient data. Lancet Oncol, 2010. 11(9): p. 835-44.

64. Martens, M.H., M. Maas, L.A. Heijnen, D.M. Lambregts, J.W. Leijtens, L.P. Stassen, S.O. Breukink, C. Hoff, 
E.J. Belgers, J. Melenhorst, R. Jansen, J. Buijsen, T.G. Hoofwijk, R.G. Beets-Tan, and G.L. Beets, Long-term Outcome of an Organ Preservation Program After Neoadjuvant Treatment for Rectal Cancer. J Natl Cancer Inst, 2016. 108(12).

65. Maas, M., R.G. Beets-Tan, D.M. Lambregts, G. Lammering, P.J. Nelemans, S.M. Engelen, R.M. van Dam, R.L. Jansen, M. Sosef, J.W. Leijtens, K.W. Hulsewe, J. Buijsen, and G.L. Beets, Wait-and-see policy for clinical complete responders after chemoradiation for rectal cancer. J Clin Oncol, 2011. 29(35): p. 463340 .

66. Smith, J.D., J.A. Ruby, K.A. Goodman, L.B. Saltz, J.G. Guillem, M.R. Weiser, L.K. Temple, G.M. Nash, and P.B. Paty, Nonoperative management of rectal cancer with complete clinical response after neoadjuvant therapy. Ann Surg, 2012. 256(6): p. 965-72.

67. Appelt, A.L., J. Ploen, I.R. Vogelius, S.M. Bentzen, and A. Jakobsen, Radiation dose-response model for locally advanced rectal cancer after preoperative chemoradiation therapy. Int J Radiat Oncol Biol Phys, 2013. 85(1): p. 74-80.

68. Fonteyne, V., S. Sadeghi, P. Ost, F. Vanpachtenbeke, P. Vuye, N. Lumen, and G. De Meerleer, Impact of changing rectal dose volume parameters over time on late rectal and urinary toxicity after high-dose intensity-modulated radiotherapy for prostate cancer: A 10-years single centre experience. Acta Oncol, 2015. 54(6): p. 854-61.

69. Gerard, J.P., A.-C. Frin, J. Doyen, F.X. Zhou, J. Gal, P. Romestaing, N. Barbet, R. Coquard, O. Chapet, E. François, S. Marcié, and K. Benezery, Organ preservation in rectal adenocarcinoma (T1) T2-T3 Nx M0. Historical overview of the Lyon Sud Nice experience using contact x-ray brachytherapy and external beam radiotherapy for 120 patients. Acta Oncol, 2015. 54(4): p. 550-556.

70. Vuong, T., S. Devic, B. Moftah, M. Evans, and E.B. Podgorsak, High-dose-rate endorectal brachytherapy in the treatment of locally advanced rectal carcinoma: technical aspects. Brachytherapy, 2005. 4(3): p. $230-5$.

71. Poon, E., B. Reniers, S. Devic, T. Vuong, and F. Verhaegen, Dosimetric characterization of a novel intracavitary mold applicator for 192Ir high dose rate endorectal brachytherapy treatment. Med Phys, 2006. 33(12): p. 4515-26.

72. Devic, S., T. Vuong, B. Moftah, M. Evans, E.B. Podgorsak, E. Poon, and F. Verhaegen, Image-guided high dose rate endorectal brachytherapy. Med Phys, 2007. 34(11): p. 4451-8.

73. Lindegaard, J., J.P. Gerard, A. Sun Myint, R. Myerson, H. Thomsen, and S. Laurberg, Whither papillon? Future directions for contact radiotherapy in rectal cancer. Clin Oncol (R Coll Radiol), 2007. 19(9): p. 738-41.

74. Sun Myint, A., A. Stewart, J. Mills, R. Sripadam, K. Whitmarsh, R. Roy, A. Franklin, A. Dhadda, and U.K.P. team, Treatment: the role of contact X-ray brachytherapy (Papillon) in the management of early rectal cancer. Colorectal Dis, 2019. 21 Suppl 1(S1): p. 45-52.

75. Gerard, J.P., A.S. Myint, O. Croce, J. Lindegaard, A. Jensen, R. Myerson, J.M. Hannoun-Levi, and S. Marcie, Renaissance of contact X-ray therapy for treating rectal cancer. Expert Rev Med Devices, 2011. 8(4): p. 483-92. 
76. Bellezzo, M., G.P. Fonseca, A.-S. Verrijssen, R. Voncken, M.R. Van den Bosch, H. Yoriyaz, B. Reniers, M. Berbée, E.J. Van Limbergen, and F. Verhaegen, A novel rectal applicator for contact radiotherapy with HDR 192Ir sources. Brachytherapy, 2018.

77. Kirisits, C., M.J. Rivard, D. Baltas, F. Ballester, M. De Brabandere, R. van der Laarse, Y. Niatsetski, P. Papagiannis, T.P. Hellebust, J. Perez-Calatayud, K. Tanderup, J.L. Venselaar, and F.A. Siebert, Review of clinical brachytherapy uncertainties: analysis guidelines of GEC-ESTRO and the AAPM. Radiother Oncol, 2014. 110(1): p. 199-212.

78. Elfrink, R.J., I.K. Kolkman-Deurloo, H.J. van Kleffens, A. Rijnders, B. Schaeken, T.H. Aalbers, W.J. Dries, and J.L. Venselaar, Determination of the accuracy of implant reconstruction and dose delivery in brachytherapy in The Netherlands and Belgium. Radiother Oncol, 2001. 59(3): p. 297-306.

79. Varian Medical Systems, P.A., CA, GammaMedplusTM iX Instructions for Use including the GammaMedplusTM 3/24 iX. 2018.

80. Gerardy, I., J. Rodenas, and S. Gallardo, Uncertainty analysis in MCNP5 calculations for brachytherapy treatment. Appl Radiat Isot, 2011. 69(8): p. 1108-11.

81. Houdek, P.V., J.G. Schwade, X. Wu, V. Pisciotta, J.A. Fiedler, C.F. Serago, A.M. Markoe, A.A. Abitbol, A.A. Lewin, P.G. Braunschweiger, and et al., Dose determination in high dose-rate brachytherapy. Int J Radiat Oncol Biol Phys, 1992. 24(4): p. 795-801.

82. Minamisawa, R.A., R.A. Rubo, R.M. Seraide, J.R. Rocha, and A. Almeida, Direct measurement of instantaneous source speed for a HDR brachytherapy unit using an optical fiber based detector. Med Phys, 2010. 37(10): p. 5407-11.

83. Fonseca, G.P., R.A. Rubo, R.A. Minamisawa, G.R. dos Santos, P.C. Antunes, and H. Yoriyaz, Determination of transit dose profile for a (192)Ir HDR source. Med Phys, 2013. 40(5): p. 051717.

84. Abbe, T., Notes on the physiologic and the therapeutic action of radium. 1904, [Washington].

85. Beck, J.C., Experiments with radium in mome nose, throat and ear diseases. The Laryngoscope, 1904. 14(12): p. 897-915.

86. Soddy, F., The Therapeutic Applications Of Radium: Methods And Results. The British Medical Journal, 1909. 1(2525): p. 1250-1251.

87. Quimby, E.H., The background of radium therapy in the United States, 1906-1956. The American journal of roentgenology, radium therapy, and nuclear medicine, 1956. 75(3): p. 443-50.

88. Simon, N., IRIDIUM 192 AS A RADIUM SUBSTITUTE. The American journal of roentgenology, radium therapy, and nuclear medicine, 1965. 93: p. 170-8.

89. Rune, W., Remotely-Controlled Afterloading Radiotherapy Apparatus (A Preliminary Report). Physics in Medicine and Biology, 1962. 7(2): p. 225-228.

90. Henschke, U.K., B.S. Hilaris, and G.D. Mahan, Remote Afterloading with Intracavitary Applicators. Radiology, 1964. 83(2): p. 344-345.

91. Henschke, U.K., B.S. Hilaris, and G.D. Mahan, INTRACAVITARY RADIATION THERAPY OF CANCER OF THE UTERINE CERVIX BY REMOTE AFTERLOADING WITH CYCLING SOURCES. American Journal of Roentgenology, 1966. 96(1): p. 45-51. 
92. Rivard, M.J., J.L. Venselaar, and L. Beaulieu, The evolution of brachytherapy treatment planning. Med Phys, 2009. 36(6): p. 2136-53.

93. Thomadsen, B.R., J.F. Williamson, M.J. Rivard, and A.S. Meigooni, Anniversary Paper: Past and current issues, and trends in brachytherapy physics Brachy Anniversary Paper. Medical Physics, 2008. 35(10): p. 4708-4723.

94. Yu, Y., L.L. Anderson, Z. Li, D.E. Mellenberg, R. Nath, M.C. Schell, F.M. Waterman, A. Wu, and J.C. Blasko, Permanent prostate seed implant brachytherapy: Report of the American Association of Physicists in Medicine Task Group No. 64. Medical Physics, 1999. 26(10): p. 2054-2076.

95. Grimm, P. and J. Sylvester, Advances in brachytherapy. Reviews in urology, 2004. 6(Suppl 4): p. 37-48.

96. Thomadsen, B.R., B. Thomadsen, M.J. Rivard, W.M. Butler, and A.A.o.P.i. Medicine, Brachytherapy Physics. second ed. 2005: American Association of Physicists in Medicine.

97. Pötter, R., J. Lindegaard, C. Kirisits, and K. Tanderup, A European study in MRI-guided brachytherapy in locally advanced cervical cancerEMBRACE. Version 17-01-2008.[cited 2019 Dec 5]. Available from: https://www.embracestudy.dk/UserUpload/PublicDocuments/EmbraceProtocol.pdf

98. Paterson, R. and H.M. Parker, A Dosage System for Gamma Ray Therapy. The British Journal of Radiology, 1934. 7(82): p. 592-632.

99. Paterson, R. and H.M. Parker, A Dosage System for Interstitial Radium Therapy. The British Journal of Radiology, 1938. 11(124): p. 252-266.

100. Quimby, E.H., Dosage Table for Linear Radium Sources. Radiology, 1944. 43(6): p. 572-577.

101. Quimby, E.H. and V. Castro, The calculation of dosage in interstitial radium therapy. The American journal of roentgenology, radium therapy, and nuclear medicine, 1953. 70(5): p. 739-49.

102. Pierquin, B., D.J. Chassagne, M.C. Chahbazian, and J.F. Wilson, Brachytherapy. 1978: W.H. Green, St Louis, MO,.

103. Nath, R., L.L. Anderson, G. Luxton, K.A. Weaver, J.F. Williamson, and A.S. Meigooni, Dosimetry of interstitial brachytherapy sources: recommendations of the AAPM Radiation Therapy Committee Task Group No. 43. Medical Physics, 1995. 22(2): p. 209-234.

104. Kutcher, G.J., L. Coia, M. Gillin, W.F. Hanson, S. Leibel, R.J. Morton, J.R. Palta, J.A. Purdy, L.E. Reinstein, and G.K. Svensson, Comprehensive QA for radiation oncology: report of AAPM radiation therapy committee task group 40. Medical physics, 1994. 21(4): p. 581-618.

105. Varian Medical Systems, P.A., CA, Guidelines for Commissioning Ring Applicators for Use with Bravos Afterloaders. 2018.

106. Steenhuizen, J., M.C. Harbers, A. Hoffmann, A.d. Leeuw, A. Rijnders, and M. Unipan. NCS Report 30: Code of Practice for Quality Assurance of Brachytherapy with Ir-192 Afterloaders. 2018.

107. Wagner, D., M. Hermann, and A. Hille, In vivo dosimetry with alanine/electron spin resonance dosimetry to evaluate the urethra dose during high-dose-rate brachytherapy. Brachytherapy, 2017. 16(4): p. 815821 .

108. Melchert, C., T. Soror, and G. Kovacs, Quality assurance during interstitial brachytherapy: in vivo dosimetry using MOSFET dosimeters. Journal of contemporary brachytherapy, 2018. 10(3): p. 232-237. 
109. Carrara, M., A. Romanyukha, C. Tenconi, D. Mazzeo, A. Cerrotta, M. Borroni, D. Cutajar, M. Petasecca, M. Lerch, J. Bucci, A. Richetti, S. Presilla, C. Fallai, G. Gambarini, E. Pignoli, and A. Rosenfeld, Clinical application of MOSkin dosimeters to rectal wall in vivo dosimetry in gynecological HDR brachytherapy. Physica Medica, 2017. 41: p. 5-12.

110. Cygler, J.E., A. Saoudi, G. Perry, C. Morash, and C. E, Feasibility study of using MOSFET detectors for in vivo dosimetry during permanent low-dose-rate prostate implants. Radiotherapy and Oncology, 2006. 80(3): p. 296-301.

111. Romanyukha, A., M. Carrara, D. Mazzeo, C. Tenconi, T. Al-Salmani, J. Poder, D. Cutajar, I. Fuduli, M. Petasecca, J. Bucci, A. Cerrotta, B. Pappalardi, F. Piccolo, E. Pignoli, and A. Rosenfeld, An innovative gynecological HDR brachytherapy applicator system for treatment delivery and real-time verification. Physica Medica, 2019. 59: p. 151-157.

112. Alecu, R. and M. Alecu, In-vivo rectal dose measurements with diodes to avoid misadministrations during intracavitary high dose rate brachytherapy for carcinoma of the cervix. Medical Physics, 1999. 26(5): p. 768-770.

113. Allahverdi, M., M. Sarkhosh, M. Aghili, R. Jaberi, A. Adelnia, and G. Geraily, Evaluation of treatment planning system of brachytherapy according to dose to the rectum delivered. Radiation protection dosimetry, 2012. 150(3): p. 312-5.

114. McCurdy, B.M., K. Luchka, and S. Pistorius, Dosimetric investigation and portal dose image prediction using an amorphous silicon electronic portal imaging device. Med Phys, 2001. 28(6): p. 911-24.

115. McDermott, L.N., R.J. Louwe, J.J. Sonke, M.B. van Herk, and B.J. Mijnheer, Dose-response and ghosting effects of an amorphous silicon electronic portal imaging device. Med Phys, 2004. 31(2): p. 285-95.

116. Taylor, R.E. and D.W. Rogers, EGSnrc Monte Carlo calculated dosimetry parameters for 192Ir and 169Yb brachytherapy sources. Med Phys, 2008. 35(11): p. 4933-44.

117. Tyner, E., B. McClean, P. McCavana, and S. af Wetterstedt, Experimental investigation of the response of an a-Si EPID to an unflattened photon beam from an Elekta Precise linear accelerator. Med Phys, 2009. 36(4): p. 1318-29.

118. McDermott, L.N., S.M. Nijsten, J.J. Sonke, M. Partridge, M. van Herk, and B.J. Mijnheer, Comparison of ghosting effects for three commercial a-Si EPIDs. Med Phys, 2006. 33(7): p. 2448-51.

119. Podesta, M., S.M. Nijsten, L.C. Persoon, S.G. Scheib, C. Baltes, and F. Verhaegen, Time dependent pretreatment EPID dosimetry for standard and FFF VMAT. Phys Med Biol, 2014. 59(16): p. 4749-68.

120. Parent, L., J. Seco, P.M. Evans, A. Fielding, and D.R. Dance, Monte Carlo modelling of a-Si EPID response: the effect of spectral variations with field size and position. Med Phys, 2006. 33(12): p. 4527-40.

121. Greer, P.B., P. Vial, L. Oliver, and C. Baldock, Experimental investigation of the response of an amorphous silicon EPID to intensity modulated radiotherapy beams. Med Phys, 2007. 34(11): p. 4389-98.

122. Fonseca, G.P., G. Landry, B. Reniers, A. Hoffmann, R.A. Rubo, P.C. Antunes, H. Yoriyaz, and F. Verhaegen, The contribution from transit dose for 192Ir HDR brachytherapy treatments. Physics in Medicine and Biology, 2014. 59(7): p. 1831-44. 
123. Podesta, M., S.M. Nijsten, J. Snaith, M. Orlandini, T. Lustberg, D. Emans, T. Aland, and F. Verhaegen, Measured vs simulated portal images for low MU fields on three accelerator types: possible consequences for 2D portal dosimetry. Med Phys, 2012. 39(12): p. 7470-9.

124. Ballester, F., D. Granero, J. Perez-Calatayud, C.S. Melhus, and M.J. Rivard, Evaluation of high-energy brachytherapy source electronic disequilibrium and dose from emitted electrons. Med Phys, 2009. 36(9): p. $4250-6$.

125. Granero, D., C. Candela-Juan, J. Vijande, F. Ballester, J. Perez-Calatayud, D. Jacob, and F. Mourtada, Technical Note: Dosimetry of Leipzig and Valencia applicators without the plastic cap. Med Phys, 2016. 43(5): p. 2087.

126. Ballester, F., V. Puchades, J.L. Lluch, M.A. Serrano-Andres, Y. Limami, J. Perez-Calatayud, and E. Casal, Technical note: Monte-Carlo dosimetry of the HDR 12i and Plus 192Ir sources. Med Phys, 2001. 28(12): p. 2586-91.

127. S.Y.F. Chu, L.P.E., and R.B. Firestone, WWW Table of Radioactive Isotopes database version 1999-02-28 from URL http://nucleardata.nuclear.lu.se/nucleardata/toi/.

128. Taylor, R.E., G. Yegin, and D.W. Rogers, Benchmarking brachydose: Voxel based EGSnrc Monte Carlo calculations of TG-43 dosimetry parameters. Med Phys, 2007. 34(2): p. 445-57.

129. GammaMed, Plus, HDR, Carleton University database. version 2008-08-29 from URL http://www.physics.carleton.ca/clrp/seed_database/Ir192_HDR/GammaMed_Plus.

130. Goorley, T., M. James, T. Booth, F. Brown, J. Bull, L.J. Cox, J. Durkee, J. Elson, M. Fensin, R.A. Forster, J. Hendricks, H.G. Hughes, R. Johns, B. Kiedrowski, R. Martz, S. Mashnik, G. McKinney, D. Pelowitz, R. Prael, J. Sweezy, L. Waters, T. Wilcox, and T. Zukaitis, Features of MCNP6. Annals of nuclear energy., 2016. 87: p. 772-783.

131. Dale, R.G., The radiobiology of Papillon-type treatments. Clin Oncol (R Coll Radiol), 2007. 19(9): p. 64954.

132. Myint, A.S., Novel radiation techniques for rectal cancer. J Gastrointest Oncol, 2014. 5(3): p. 212-7.

133. Micke, A., D.F. Lewis, and X. Yu, Multichannel film dosimetry with nonuniformity correction. Med Phys, 2011. 38(5): p. 2523-34.

134. Jeong, J., C.A. Barker, M. Zaider, and G.N. Cohen, Impact of source position on high-dose-rate skin surface applicator dosimetry. Brachytherapy, 2016. 15(5): p. 650-60.

135. Low, D.A., W.B. Harms, S. Mutic, and J.A. Purdy, A technique for the quantitative evaluation of dose distributions. Med Phys, 1998. 25(5): p. 656-61.

136. Low, D.A. and J.F. Dempsey, Evaluation of the gamma dose distribution comparison method. Med Phys, 2003. 30(9): p. 2455-64.

137. Croce, O., S. Hachem, E. Franchisseur, S. Marcié, J.-P. Gérard, and J.-M. Bordy, Contact radiotherapy using a 50kV X-ray system: Evaluation of relative dose distribution with the Monte Carlo code PENELOPE and comparison with measurements. Radiation Physics and Chemistry, 2012. 81(6): p. 609-617.

138. Sun Myint, A., Renaissance of contact radiotherapy with RT 50 papillon machine: Preliminary data on first 100 patients treated at Clatterbridge. Journal of Clinical Oncology, 2011. 29(15_suppl): p. e14149. 
139. Sun Myint, A., H. Wong, K. Whitmarsh, R. Sripadam, K. Perkins, D.M. Pritchard, F.M. Smith, P. Rooney, S.W. Gollins, C. Rao, M.J. Hershman, and Z. Fekete, Dose escalation using contact X-ray brachytherapy (Papillon) for rectal cancer: Does it improve the chance of organ preservation? British Journal of Radiology, 2017. 90(1080).

140. Bellezzo, M., G.P. Fonseca, A.S. Verrijssen, R. Voncken, M.R. Van den Bosch, H. Yoriyaz, B. Reniers, M. Berbee, E.J. Van Limbergen, and F. Verhaegen, A novel rectal applicator for contact radiotherapy with HDR (192)Ir sources. Brachytherapy, 2018. 17(6): p. 1037-1044.

141. Perez-Calatayud, J., F. Ballester, R.K. Das, L.A. DeWerd, G.S. Ibbott, A.S. Meigooni, Z. Ouhib, M.J. Rivard, R.S. Sloboda, and J.F. Williamson, Dose calculation for photon-emitting brachytherapy sources with average energy higher than 50keV: Report of the AAPM and ESTRO. Medical Physics, 2012. 39(5): p. 29042929.

142. Fonseca, G.P., A.C. Tedgren, B. Reniers, J. Nilsson, M. Persson, H. Yoriyaz, and F. Verhaegen, Dose specification for 192Ir high dose rate brachytherapy in terms of dose-to-water-in-medium and dose-tomedium-in-medium. Phys Med Biol, 2015. 60(11): p. 4565-79.

143. Williamson, J.F., Monte Carlo evaluation of kerma at a point for photon transport problems. Med Phys, 1987. 14(4): p. 567-76.

144. Scapin, M., Mechanical characterization and modeling of the heavy tungsten alloy IT180. International Journal of Refractory Metals and Hard Materials, 2015. 50: p. 258-268.

145. EBT-XD Specification and user guide. [cited 2019 July 1]; Available from: http://www.gafchromic.com/documents/EBTXD_Specifications_Final.pdf.

146. Grams, M.P., J.M. Gustafson, K.M. Long, and L.E. de los Santos, Technical Note: Initial characterization of the new EBT-XD Gafchromic film. Med Phys, 2015. 42(10): p. 5782-6.

147. Perez-Calatayud, J., F. Ballester, M.A. Serrano-Andres, V. Puchades, J.L. Lluch, Y. Limami, and F. Casal, Dosimetry characteristics of the Plus and 12i Gammamed PDR 192Ir sources. Med Phys, 2001. 28(12): p. 2576-85.

148. Patel, N.P., B. Majumdar, and V. Vijayan, Comparative dosimetry of GammaMed Plus high-dose rate Ir brachytherapy source. J Med Phys, 2010. 35(3): p. 137-43.

149. Varian Medical Systems, P.A., CA, Varisource afterloader features sheet. 2015.

150. Varian Medical Systems, P.A., CA, Bravos Instructions for Use, Version 1.0. 2018.

151. Varian Medical Systems, P.A., CA, Varian Brachytherapy Applicators and Accessories. 2011.

152. Varian Medical Systems, P.A., CA, Acuros BV Algorithm Reference Guide_v11. 2011.

153. Lavery, I.C., I.T. Jones, F.L. Weakley, J.P. Saxton, V.W. Fazio, and D.G. Jagelman, Definitive management of rectal cancer by contact (endocavitary) irradiation. Dis Colon Rectum, 1987. 30(11): p. 835-8.

154. Gerard, J.P., L. Ayzac, R. Coquard, P. Romestaing, J.M. Ardiet, F.P. Rocher, N. Barbet, J.L. Cenni, and J.C. Souquet, Endocavitary irradiation for early rectal carcinomas T1 (T2). A series of 101 patients treated with the Papillon's technique. Int J Radiat Oncol Biol Phys, 1996. 34(4): p. 775-83. 
155. Gerard, J.P., P. Roy, R. Coquard, N. Barbet, P. Romestaing, L. Ayzac, J.M. Ardiet, and J.C. Thalabard, Combined curative radiation therapy alone in (T1) T2-3 rectal adenocarcinoma: a pilot study of 29 patients. Radiother Oncol, 1996. 38(2): p. 131-7.

156. Maingon, P., S. Guerif, R. Darsouni, S. Salas, I. Barillot, A. d'Hombres, M.C. Bone-Lepinoy, J. Fraisse, and J.C. Horiot, Conservative management of rectal adenocarcinoma by radiotherapy. Int J Radiat Oncol Biol Phys, 1998. 40(5): p. 1077-85.

157. Lavertu, S., S.E. Schild, L.L. Gunderson, M.G. Haddock, and J.A. Martenson, Endocavitary radiation therapy for rectal adenocarcinoma: 10-year results. Am J Clin Oncol, 2003. 26(5): p. 508-12.

158. Coatmeur, O., G. Truc, I. Barillot, J.C. Horiot, and P. Maingon, Treatment of T1-T2 rectal tumors by contact therapy and interstitial brachytherapy. Radiother Oncol, 2004. 70(2): p. 177-82.

159. Gerard, J.P., O. Chapet, C. Nemoz, J. Hartweig, P. Romestaing, R. Coquard, N. Barbet, P. Maingon, M. Mahe, J. Baulieux, C. Partensky, M. Papillon, O. Glehen, B. Crozet, J.P. Grandjean, and P. Adeleine, Improved sphincter preservation in low rectal cancer with high-dose preoperative radiotherapy: the lyon R96-02 randomized trial. J Clin Oncol, 2004. 22(12): p. 2404-9.

160. Gerard, J.P., C. Ortholan, K. Benezery, A. Ginot, J.M. Hannoun-Levi, E. Chamorey, D. Benchimol, and E. Francois, Contact X-ray therapy for rectal cancer: experience in Centre Antoine-Lacassagne, Nice, 20022006. Int J Radiat Oncol Biol Phys, 2008. 72(3): p. 665-70.

161. Christoforidis, D., M.P. McNally, S.L. Jarosek, R.D. Madoff, and C.O. Finne, Endocavitary contact radiation therapy for ultrasonographically staged T1 N0 and T2 N0 rectal cancer. Br J Surg, 2009. 96(4): p. 430-6.

162. Ortholan, C.P.D., P.M.D. Romestaing, O.P.D. Chapet, and J.P.M.D. Gerard, Correlation in Rectal Cancer Between Clinical Tumor Response After Neoadjuvant Radiotherapy and Sphincter or Organ Preservation: 10-Year Results of the Lyon R 96-02 Randomized Trial. International Journal of Radiation Oncology, Biology, Physics, 2012. 83(2): p. e165-e171.

163. Varian Medical Systems, P.A., CA, GammaMedplus HDR and PDR User Guide. 2006.

164. Stern, R.L. and T. Liu, Dwell position inaccuracy in the Varian GammaMed HDR ring applicator. J Appl Clin Med Phys, 2010. 11(4): p. 3158.

165. Myint, A.S. and J.P. Gerard, Minimally invasive contact X-ray brachytherapy as an alternative option in patients with rectal cancer not suitable for bespoke surgical resection. Mini-invasive Surgery, 2018. 2(10).

166. Verrijssen, A., M. Bellezzo, A. Habr-Gama, R. Perez, J. Guillem, K. Bujko, R. Houben, F. Verhaegen, M. Berbee, and E. Van Limbergen, EP-1472: Microscopic extension of residual rectal tumor mass postneoadjuvant chemoradiation: a meta-analysis. Radiotherapy and Oncology, 2018. 127(Supplement 1): p. S799-S799.

167. Reniers, B., D. Liu, T. Rusch, and F. Verhaegen, Calculation of relative biological effectiveness of a lowenergy electronic brachytherapy source. Phys Med Biol, 2008. 53(24): p. 7125-35.

168. Wojcicka, J.B., R. Yankelevich, F. Trichter, and D.P. Fontenla, Comparison of the transit dose components and source kinematics of three high dose rate afterloading systems. Med Dosim, 1999. 24(1): p. 61-5.

169. Awunor, O.A., B. Dixon, and C. Walker, Direct reconstruction and associated uncertainties of 192Ir source dwell positions in ring applicators using gafchromic film in the treatment planning of HDR brachytherapy cervix patients. Phys Med Biol, 2013. 58(10): p. 3207-25. 
170. Liang, L.H., N. Tomic, T. Vuong, S. Aldelaijan, H. Bekerat, F. DeBlois, J. Seuntjens, and S. Devic, Physics aspects of the Papillon technique-Five decades later. Brachytherapy, 2018. 17(1): p. 234-243.

171. Rickey, D.W., D. Sasaki, and J. Bews, A quality assurance tool for high-dose-rate brachytherapy. Med Phys, 2010. 37(6): p. 2525-32.

172. Suchowerska, N., M. Jackson, J. Lambert, Y.B. Yin, G. Hruby, and D.R. McKenzie, Clinical trials of a urethral dose measurement system in brachytherapy using scintillation detectors. Int J Radiat Oncol Biol Phys, 2011. 79(2): p. 609-15. 



\section{Valorization}



Brachytherapy has been under continuous development since its origin, as it has been proven an effective technique for cancer treatment. Efforts have been made to improve precision and accuracy of treatment delivery, including several steps on the treatment workflow, from applicators design to treatment delivery verification and in vivo dosimetry, as mentioned in Figure 1.1. This thesis focus on advanced methods as part of an effort to overcome these technical challenges aiming to provide tools that will help move towards more accurate dose delivery.

The studies in this thesis were performed in collaboration between 2 countries, Brazil and the Netherlands, with a strong input from a multidisciplinary team including engineers, medical doctors, physicists and radiotherapy technologist, always having the quality improvement of patient care as the main goal.

This thesis can be divided into 3 main novel subjects:

\section{Brachytherapy source tracking using an imaging panel}

Imaging panels are commonly used for treatment verification in external beam radiotherapy, however, their use in brachytherapy is still incipient. Studies in this thesis showed that an imaging panel is not only capable of 3D tracking a brachytherapy source in real time, but also detect errors (such as wrong dwell time or dwell position) that would be unnoticed in standard practice. Moreover, it was shown that imaging panels can be also used as a precise and accurate method for brachytherapy applicators commissioning, measuring not only source dwell positions and dwell times, but comparing them with reference treatment plans to detect errors.

\section{Development of a rectal applicator for contact brachytherapy}

The standard care of treatment for rectal cancer includes surgery, which may have undesirable side effects. In some cases, surgery can be omitted in treatments including contact x-ray brachytherapy techniques, however, such techniques are not often adopted because of the lack of planning tools and the relative high cost of the aforementioned devices. The development of a rectal applicator able to generate a dose distribution similar to those of CBX devices using HDR ${ }^{192}$ Ir sources, holds the potential to reduce the number of patients having surgery during a rectal cancer treatment, therefore improving patient quality of life. 


\section{Evaluation of an afterloader treatment limitations and transit time}

The afterloader is an essential part of HDR brachytherapy. Its precision and accuracy for dwell times and dwell positions directly impacts the resulting dose distribution and, consequently, treatment outcome. A thorough evaluation of the new Bravos afterloader released by Varian Medical Systems also shows how precise and accurate it can be during treatment delivery. Moreover, the transit time measured with a high-speed camera allows a precise transit dose simulation using the instantaneous source speed instead of average source speed profiles, which are especially relevant for treatments using multiple channels such as HDR prostate treatments.

\section{Clinical relevance}

Patient safety and quality of life are of the uttermost importance for any healthcare center. Therefore, medical research centers are always working to improve their treatment workflow and installations aiming to provide the best experience possible for the patients. Nevertheless, brachytherapy is a treatment modality prone to human errors and it lacks the treatment verification tools to detect them in case a mistake happens during the treatment. Efforts have been made towards in vivo dosimetry and real time treatment verification, but there is no solution commercially available yet.

For this reason, a proper system commissioning is paramount to a successful treatment delivery. The work on the pretreatment verification system is a first step moving towards real time treatment verification.

The IrIS system has a friendly interface and allows the user to perform several quality assurance measurements in a fraction of the time that would be required if radiochromic films were used, allowing a better applicator evaluation.

The MAASTRO applicator holds the potential to be a cost-effective alternative to contact $\mathrm{x}$-ray brachytherapy devices since it does not require any extra tool other than those that an oncology center that works with brachytherapy already has. Moreover, the possibility to control the shape of the dose distribution using a treatment planning system is an advantage of the MAASTRO applicator when compared to CBX devices. 


\section{Societal relevance}

The patient is the first to benefit from a more precise and accurate radiotherapy treatment. All studies presented in this thesis aim to improve brachytherapy precision and accuracy, which will reflect on the quality of the treatment received by the patient.

The MAASTRO applicator, which is the main subject of this thesis, should provide one more treatment modality for rectal cancer patients, potentially reducing the number of surgeries and, consequently, improving quality of life. Moreover, due to its expected low cost, the MAASTRO applicator may be especially societal relevant in developing countries.

\section{Commercial relevance}

The MAASTRO applicator was co-developed with Varian Medical Systems and will become a commercially available product in the coming years. The first clinical trials are expected to start in 2021. 



\section{List of Publications}





\section{Articles in press}

Bellezzo, M., G.P. Fonseca, R. Voncken, A.-S. Verrijssen, R. Voncken, C. Van Beveren , E. Roelofs, H. Yoriyaz, B. Reniers, E.J. Van Limbergen, M. Berbee, and F. Verhaegen, Advanced design, simulation, and dosimetry of a novel rectal applicator for contact brachytherapy with a conventional HDR ${ }^{192}$ Ir source. Brachytherapy, 2020.

\section{Original Articles}

Verrijssen, A.-S, J. Guillem, R., K. Bujko, N. Guedj, A. Habr-Gama, R. Houben, D. Goudkade, J. Melenhorst, J. Buijsen, B. Vanneste, H. I Grabsch, M. Bellezzo, G. P. Fonseca, F. Verhaegen, M. Berbee, E. J. Van Limbergen

Bellezzo, M., J.A. Baeza, R. Voncken, B. Reniers, F. Verhaegen, and G.P. Fonseca, Mechanical evaluation of the Bravos afterloader system for HDR brachytherapy. Brachytherapy, 2019. 18(6): p. 852-862.

Verrijssen, A.-S., T. Opbroek, M. Bellezzo, G.P. Fonseca, F. Verhaegen, J.-P. Gerard, A.S. Myint, E.J. Van Limbergen, and M. Berbee, Corrigendum to A systematic review comparing radiation toxicity after various endorectal techniques, by Verrijssen et al., Brachytherapy. 2019 Jan - Feb;18(1):71-86.e5. Brachytherapy, 2019. 18(3): p. 427-427.

Soares Lopes Branco, I., F.c.A. Lima, P.C. Guimaraes Antunes, H. Yoriyaz, M. Bellezzo, G. Paiva Fonseca, E. Borguezan Neto, A. Colello Bruno, M.l. Lisboa Roca Santo, G. Lazzaro Barbi, L. Federiche Borges, E.C. Bertucci, G. Viani Arruda, and J. Fernandes Pavoni, Desenvolvimento de software de calculo de dose pontual em Braquiterapia baseado em simulacoes de Monte Carlo. Revista Brasileira de Fisica Medica, 2018. 12(1): p. 2.

Verrijssen, A.-S., T. Opbroek, M. Bellezzo, G.P. Fonseca, F. Verhaegen, J.-P. Gerard, A. Sun Myint, E.J. Van Limbergen, and M. Berbee, A systematic review comparing radiation toxicity after various endorectal techniques. Brachytherapy, 2019. 18(1): p. 71-86.

Bellezzo, M., G.P. Fonseca, A.-S. Verrijssen, R. Voncken, M.R. Van den Bosch, H.l. Yoriyaz, B. Reniers, M. Berbee, E.J. Van Limbergen, and F. Verhaegen, A novel rectal applicator for contact radiotherapy with HDR 192Ir sources. Brachytherapy, 2018. 17(6): p. 1037-1044.

Camps, S.M., M. Costa, E. Stevens, P.G. Sanches, G. Paiva Fonseca, M. Bellezzo, and F. Verhaegen, Evaluation of optical localization in the visible band for ultrasound guidance in radiotherapy using a robotic arm. Journal of Cancer Control and Treatment, 2017. 30(5): p. 319-329.

Fonseca, G.P., M. Podesta, M. Bellezzo, M., R. Van den Bosch, L. Lutgens, B.G.L. Vanneste, R. Voncken, E.J. Van Limbergen, B. Reniers, and F. Verhaegen, Online pretreatment verification of high-dose rate brachytherapy using an imaging panel. Physics in Medicine 
\& Biology, 2017. 62(13): p. 5440-5461.

Fonseca, G.P., B. Reniers, G. Landry, S. White, M. Bellezzo, P.C.G. Antunes, C.P. de Sales, E. Welteman, H. Yoriyaz, and F. Verhaegen, A medical image-based graphical platformFeatures, applications and relevance for brachytherapy. Brachytherapy, 2014. 13(6): p. 632-639.

\section{Abstracts/Conference papers}

Bellezzo, M., G.P. Fonseca, R. Voncken, A.-S. Verrijssen, B. Reniers, M. Berbee, E.J. Van Limbergen, and F. Verhaegen, Contact Radiotherapy with a Rectal Brachytherapy Applicator Using 192Ir HDR Sources. Brachytherapy, 2019. 18(3 Supplement): p. S39-S40.

Verrijssen, A., M. Bellezzo, A. Habr-Gama, R. Perez, J. Guillem, K. Bujko, R. Houben, F. Verhaegen, M. Berbee, and E. Van Limbergen, EP-1472: Microscopic extension of residual rectal tumor mass post-neoadjuvant chemoradiation: a meta-analysis. Radiotherapy and Oncology: Supplement 1, 2018. 127(Supplement 1): p. S799-S799.

Bellezzo, M., M. Berbee, B. Reniers, A. Verrijssen, G. Fonseca, E. Van Limbergen, and F. Verhaegen, EP-2249: A novel rectal applicator for contact radiotherapy with 192Ir HDR sources. Radiotherapy and Oncology: Supplement 1, 2018. 127(Supplement 1): p. S1243S1244.

Verrijssen, A., T. Opbroek, F. Verhaegen, M. Bellezzo, G. Paiva Fonseca, E. Van Limbergen, and M. Berbee, EP-1460: A systematic review comparing radiation toxicity after various endorectal radiation techniques. Radiotherapy and Oncology: Supplement 1, 2018. 127(Supplement 1): p. S792-S793.

Van den Bosch, M., G. Fonseca, R. Voncken, M. Bellezzo, and F. Verhaegen, PO-1023: Verifying brachytherapy applicator models using an imaging panel. Radiotherapy and Oncology: Supplement 1, 2018. 127(Supplement 1): p. S574-S574.

Fonseca, G., M. Podesta, M. Bellezzo, B. Reniers, and F. Verhaegen, PO-0945: Pretreatment verification for brachytherapy. Radiotherapy and Oncology: Supplement 1, 2017. 123(Supplement 1): p. S523-S524.

Bellezzo, M.; Nascimento, E. ; Yoriyaz, H. . Desenvolvimento de um software de monte carlo para transporte de fótons em estruturas de voxels usando unidades de placas gráficas. in: XIV International Symposium on Solid State Dosimetry, 2014, Cuscu. libro de resumenes xiv isssd, 2014, 2014

Nahuel Cardenas, J.P., T.M. Filho, A.R. Petri, R.A. dos Santos, J.F.T. Martins, D.V.S. Carvalho, T. Alvarenga, M. Bellezzo, G. Laranjo, M. Lima, P. Oliveira, M. Costa Pereira. Experimental and MCNP studies of Paraffin and Polyethylene in Neutron Moderation and BF3 Detector 
Efficiency. 3rd International Conference on Advancements in Nuclear Instrumentation, Measurement Methods and their Applications (ANIMMA), 2013, IEEE. p. 1-5

Filho, T.M., J.P. Nahuel Cardenas, A.R. Petri, R.A. dos Santos, J.F.T. Martins, D.V.S. Carvalho, T. Alvarenga, M. Bellezzo, G. Laranjo, M. Lima, P. Oliveira, C. M. Da Costa Pereira. Am-Be neutron irradiator used for nuclear instrumentation training. 3rd International Conference on Advancements in Nuclear Instrumentation, Measurement Methods and their Applications (ANIMMA), 2013, IEEE. p. 1-5 



\section{Curriculum vitae}



Born in Guarulhos, Brazil, Murillo Bellezzo discovered his interest in math after being a gold medallist in the São Paulo state math olympics. After being awarded in the brazilian physics olympics, he was invited to an advanced school of physics, hosted by the University of São Paulo (USP), where he obtained his degree in physics in 2011. He obtained his master's degree in nuclear technology in 2014 from USP, under the supervision Prof. Dr Hélio Yoriyaz, and in 2015 he started a Ph.D in Brazil under supervision Prof. Dr Hélio Yoriyaz, joining Maastro (Maastricht University (MU) the Netherlands) research team in 2016 under supervision of Prof. Dr. Frank Verhaegen. He concluded his double Ph.D, USP and MU, in 2020 performing research activities in both countries Brazil and the Netherlands. 
